\title{
Life Cycle Assessment of Direct Air Carbon Capture and Storage with Low-Carbon Energy Sources
}

Tom Terlouw, Karin Treyer, christian bauer, Marco Mazzotti

Submitted date: 19/05/2021 P Posted date: 21/05/2021

Licence: CC BY-NC-ND 4.0

Citation information: Terlouw, Tom; Treyer, Karin; bauer, christian; Mazzotti, Marco (2021): Life Cycle Assessment of Direct Air Carbon Capture and Storage with Low-Carbon Energy Sources. ChemRxiv. Preprint. https://doi.org/10.26434/chemrxiv.14346182.v2

Prospective energy scenarios usually rely on carbon dioxide removal (CDR) technologies to achieve the climate goals of the Paris Agreement. CDR technologies aim at removing $\mathrm{CO} 2$ from the atmosphere in a permanent way. However, the implementation of CDR technologies typically comes along with unintended environmental side-effects such as land transformation or water consumption. These need to be quantified before large-scale implementation of any CDR option by means of life cycle assessment (LCA). Direct air carbon capture and storage (DACCS) is considered to be among the CDR technologies closest to large-scale implementation, since first pilot and demonstration units have been installed and interactions with the environment are less complex than for biomass related CDR options. However, only very few LCA studies with limited scope - have been conducted so far to determine the overall life-cycle environmental performance of DACCS. We provide a comprehensive LCA of different low temperature DACCS configurations - pertaining to solid sorbent-based technology - including a global and prospective analysis.

File list (2)

SI_Life Cycle Assessment of Direct Air Carbon Capture an... (4.03 MiB) view on ChemRxiv • download file Life_Cycle_Assessment_of_Direct_Air_Carbon_Capture_... (7.28 MiB) view on ChemRxiv • download file 


\section{Life cycle assessment of direct air carbon capture and storage with low-carbon energy sources}

\section{† Supplementary Information (SI)}

Tom Terlouw ${ }^{1,2 *}$, Karin Treyer $^{1}$, Christian Bauer ${ }^{1}$, and Marco Mazzotti

${ }^{1}$ Paul Scherrer Institute, Laboratory for Energy Systems Analysis,

5232 Villigen PSI, Switzerland

${ }^{2}$ Institute of Energy and Process Engineering,

ETH Zürich, Zürich 8092, Switzerland.

${ }^{*}$ Corresponding author

tom.terlouw@psi.ch, karin.treyer@psi.ch,christian.bauer@psi.ch,

marco.mazzotti@ipe.mavt.ethz.ch

\section{Contents}

S1. Verification of information obtained from Climeworks and corresponding contribution analysis (regarding impacts on

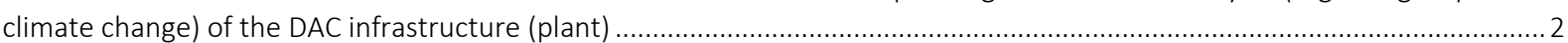

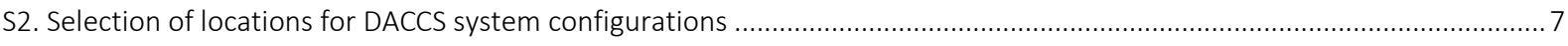

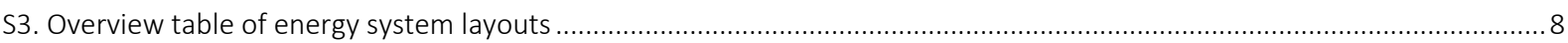

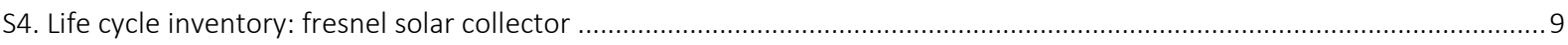

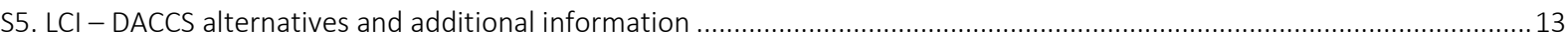

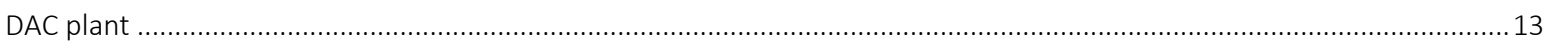

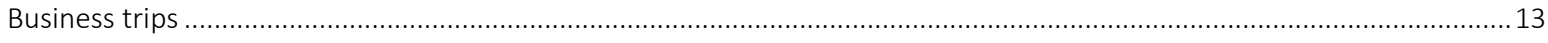

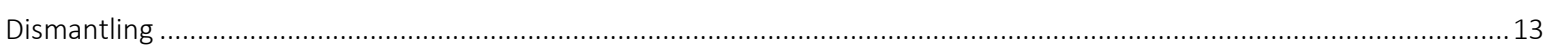

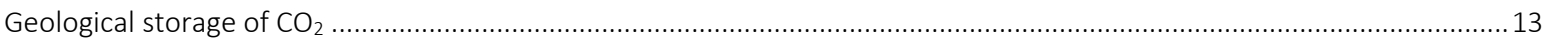

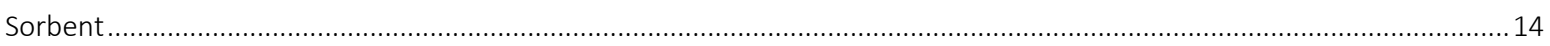

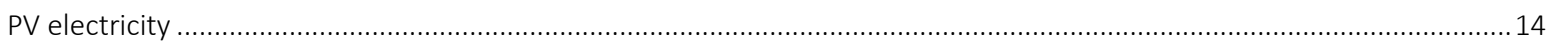

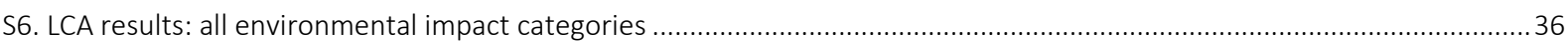

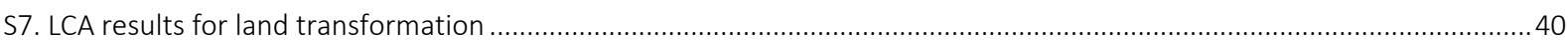

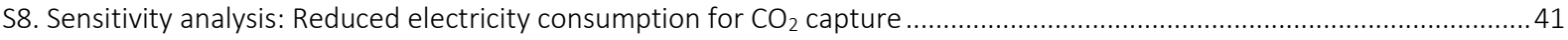

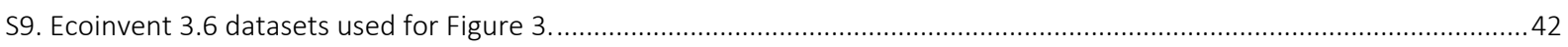

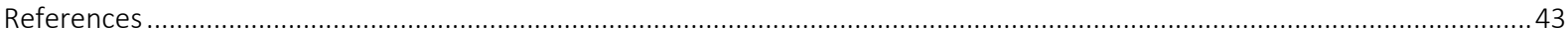

Our functional unit:

'Gross removal of 1 ton $\mathrm{CO}_{2}$ from the atmosphere via the use of a DAC plant combined with geological $\mathrm{CO}_{2}$ storage' 


\section{S1. Verification of information obtained from Climeworks and corresponding contribution analysis (regarding impacts on climate change) of the DAC infrastructure (plant)}

\section{Contribution analysis of the DAC infrastructure (plant) for today and near-term future obtained from Climeworks regarding life-cycle impacts on climate change}

Two Direct Air Capture (DAC) infrastructure specifications were received from Climeworks in terms of material and operational energy inventory: one corresponding to a current unit (4 kt $\mathrm{CO}_{2} /$ year captured), and the other for a near-term future installation ( $100 \mathrm{kt} \mathrm{CO}_{2}$ /year captured). We present a contribution analysis regarding life-cycle greenhouse gas (GHG) emissions for the DAC infrastructure (i.e. the construction of the DAC unit) for both specifications in the following figures. The figures demonstrate that the material intensity is expected to decrease for a near future installation compared to the current plant design, which is due to material savings enabled by technological improvements, while the energy consumption for the $\mathrm{CO}_{2}$ capture process will stay constant.
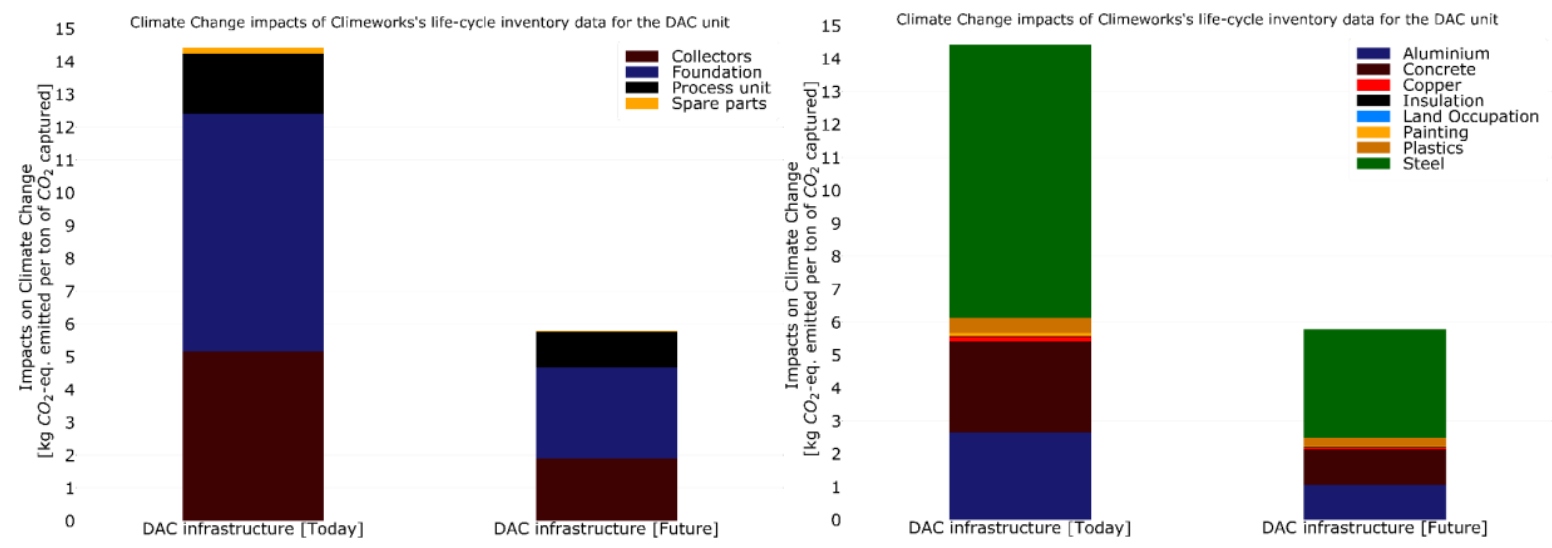

Figure A. Contribution analysis on Climate Change impacts of the main DAC components and materials per ton of $\mathrm{CO}_{2}$ captured for two scenarios: the current life cycle inventory used in Iceland (4 kt plant) and a future scenario (100 kt plant). Left panel: breakdown per main component; right panel: breakdown per main material.

\section{Verification of information obtained from Climeworks regarding DAC infrastructure material needs and generation of new life-cycle inventory}

We are not allowed to publish the complete material inventories of the DAC system we obtained from Climeworks, since Climeworks' DAC infrastructure data is confidential (a proprietary technology). Therefore, we generate a life-cycle inventory $(\mathrm{LCl})$ of the DAC unit based on freely available data on the web in order to verify the $\mathrm{LCl}$ of Climeworks. In the following calculations, we check the DAC infrastructure requirements for the $4 \mathrm{kt} \mathrm{CO}_{2} /$ year and $100 \mathrm{kt} \mathrm{CO}_{2} /$ year capture unit based on the $0.9 \mathrm{kt} \mathrm{CO}_{2}$ /year DAC plant in Hinwil (Switzerland), since this plant has already been installed and some data and figures are freely available on the web.

We assume that the DAC system consists of modular collectors for $\mathrm{CO}_{2}$ capture from air, a process unit (e.g. for preparation of sorbent and piping), a steel tank (i.e. hot water reservoir) and a hall/building (e.g. for control purposes) ${ }^{\text {a. }}$. First, we estimate that each collector is a box with a width, length and height of 2 meters (based on figures of Climeworks' website and the $w^{b} b^{b}$ ). These collector boxes are designed in a modular way, and 6 collectors fit in a 40 foot shipping container (Beuttler, Charles and Wurzbacher, 2019), which corresponds to our estimated sizes of the boxes. Further, the layout of the collector system ( $0.9 \mathrm{kt} \mathrm{CO} /$ /year captured unit) in Hinwil (Switzerland) shows the requirement of 18 collector boxes to capture $0.9 \mathrm{kt}$ $\mathrm{CO}_{2} /$ yeara . Therefore, each collector box captures $50 \mathrm{t} \mathrm{CO}$ /year (Beuttler, Charles and Wurzbacher, 2019), since the collector boxes are designed in a modular way (Beuttler, Charles and Wurzbacher, 2019). Hence, 80 collector boxes are required for a DAC unit capturing $4 \mathrm{kt} \mathrm{CO} /$ year.

We assume that the life cycle inventory of the collector box (e.g. for a fan, steel, insulation, plastics) is - in terms of material composition - similar to the life cycle inventory of a passenger vehicle (e.g. steel, motor, electronics, plastics). Such an assumption has also been made in van der Giesen et al. (van der Giesen et al., 2017). We use a $1200 \mathrm{~kg}$ compact size petrol/natural gas car as best available approximation (dataset 'market for passenger car, petrol/natural gas' in ecoinvent 3.6). The dimensions of the car are assumed to be the same as a compact sized car, a Volkswagen Golf, with a width of 1.8

\footnotetext{
${ }^{a}$ https://houseofswitzerland.org/swissstories/environment/climeworks-technology-reverse-climate-change (08.12.2020).

b https://www.nytimes.com/2019/02/12/magazine/climeworks-business-climate-change.html (07.12.2020).
} 
meter, a length of 4 meter and a height of 1.5 meter (Volkswagen, 2006). Since we calculated the total volume requirement for the collector boxes $\left(640 \mathrm{~m}^{3}\right.$, with $8 \mathrm{~m}^{3}$ per collector box), we can calculate the amounts of materials in the life cycle inventory in $\mathrm{kg}$ passenger vehicle (with a density of $\sim 110 \mathrm{~kg} / \mathrm{m}^{3}$ ) needed for the process unit. Table 1 shows the main parameters used for the calculation of our self-generated life cycle inventory.

Based on freely available figures for the DAC unit in Hinwil $(0.9 \mathrm{kt} \mathrm{CO} /$ year captured unit)a , we obtain that the DAC unit consists of 2 containers (i.e. assumed to be the process units, hence, 1 container per 9 collector boxes) with an estimated size of 12 meters length ( 6 collector boxes), 2 meters width and 2 meters height, i.e. a volume of approximately $50 \mathrm{~m}^{3}$ per process unit. Hence, we linearly scale the amount of process units needed for the $4 \mathrm{kt} \mathrm{CO}_{2} /$ year captured unit, which results in 9 process units with a total volume of $450 \mathrm{~m}^{3}$. For the sake of simplicity, we use the same approach as with the collector boxes, and assume the life cycle inventory of the process unit to be similar to the one of a passenger vehicle. Furthermore, one steel $\operatorname{tank}\left(7850 \mathrm{~kg} / \mathrm{m}^{3} \mathrm{c}\right)$ is needed (one steel tank per 18 collector boxes) for storage and is assumed to have a diameter, height and thickness of 2 meters, 6 meters and 0.02 meter, respectively.

Table 1. Main parameters used for our self-generated life cycle inventory.

\begin{tabular}{|l|r|r|r|l|}
\hline & Hinwil (Switzerland) & Current technology (4 kt) & Future technology (100 kt) & Unit \\
\hline Capacity & 0.9 & 4 & 100 & $\mathrm{kt} \mathrm{CO}_{2} /$ year \\
\hline Collector boxes & 18 & 80 & 1000 & - \\
\hline Specific capacity & & & 50 & $\mathrm{CO}_{2} \mathrm{captured} /$ \\
Land use occupation & 50 & 400 & 100 & collector box/year \\
\hline Hall area & 90 & 300 & 5000 & $\mathrm{~m}^{2}$ land \\
\hline
\end{tabular}

Further, land preparation required before installation of the DAC unit includes the conversion of grassland to industrial area, and the subsequent coverage of land with concrete (as foundation for the process unit and collectors). Viebahn et al. (Viebahn, Scholz and Zelt, 2019) reported a land occupation for the DAC plant of $90 \mathrm{~m}^{2}$ for Climeworks' plant in Hinwil. Hence, we apply a linear increase of land use occupation and use $400 \mathrm{~m}^{2}$ for the $4 \mathrm{kt} \mathrm{CO}_{2} /$ year capture unit. With a concrete layer of 1 meter, this results in a concrete volume of $400 \mathrm{~m}^{3}$ - with a reinforcement of $120 \mathrm{~kg} \mathrm{steel} / \mathrm{m}^{3}$ concrete(Deutz and Bardow, 2021) required.

According to information of Climeworks, a hall/building is installed (e.g. for control purposes). However, the dimensions of this hall/building remain unclear, also whether the land occupation of the hall/building is considered in the land occupation presented in Viebahn et al. (Viebahn, Scholz and Zelt, 2019) or not. We estimate that the building/hall occupies $75 \%$ of the total surface area (i.e. a total surface of $300 \mathrm{~m}^{2}$ ), with a lifetime of 50 years. An overview of our self-generated life-cycle inventory for the $4 \mathrm{kt} \mathrm{CO}_{2}$ /year captured unit is presented in Table 2.

For the future DAC unit, we assume that each collector box captures twice as much $\mathrm{CO}_{2}$ due to an improved capture process. This results in less material consumption, land use as well as a smaller hall/building per unit of $\mathrm{CO}_{2}$ captured. With this assumption, we are able to generate the life-cycle inventory for the near future DAC plant considering this improved performance. This life-cycle inventory is also presented in Table 2.

\footnotetext{
${ }^{c}$ https://www.engineeringtoolbox.com/metal-alloys-densities-d 50.html (08.12.2020).
} 

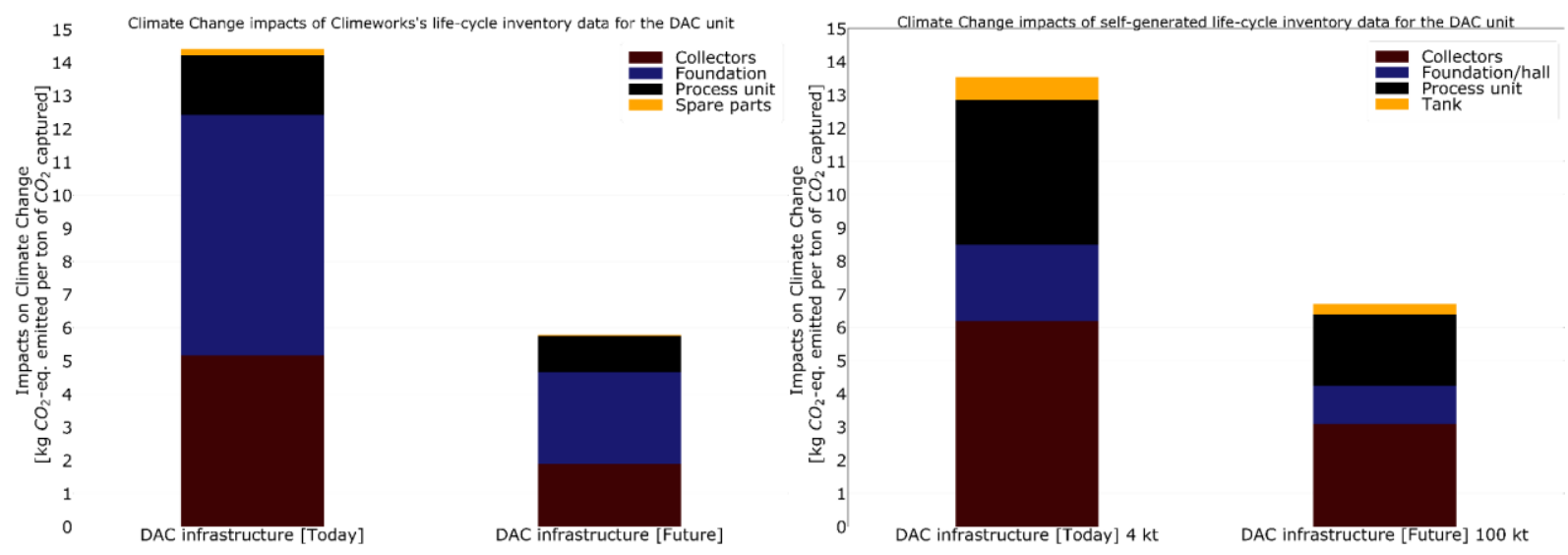

Figure B. Contribution analysis on Climate Change impacts of the main DAC components (for a DAC plant of $4 \mathrm{kt} \mathrm{CO}_{2}$ captured/year) per ton of $\mathrm{CO}_{2}$ captured generated from Climeworks's (figure on the left) inventory and our simplified calculation (figure on the right).

The DAC infrastructures approximated with our simplified calculation exhibits a Climate Change impact for the total DAC infrastructure of $13.5 \mathrm{~kg} \mathrm{CO}$-eq/t CO 2 captured and $6.7 \mathrm{~kg} \mathrm{CO}$-eq/t CO 2 captured for the $4 \mathrm{kt} \mathrm{CO}_{2}$ captured/year and $100 \mathrm{kt}$ $\mathrm{CO}_{2}$ captured/year DAC units, respectively (see Figure B). The life cycle inventory for the DAC infrastructure provided by Climeworks' results in $14.4 \mathrm{~kg} \mathrm{CO}$-eq/ $/ \mathrm{tCO}_{2}$ captured and $5.8 \mathrm{~kg} \mathrm{CO}$-eq/t CO 2 captured for the $4 \mathrm{kt} \mathrm{CO}_{2}$ captured/year and $100 \mathrm{kt} \mathrm{CO}$ captured/year DAC units, respectively (see Figure A). In other words, the difference between both approaches in terms of Climate Change impact is very small: $\sim 1 \mathrm{~kg} \mathrm{CO}$-eq/t $\mathrm{CO}_{2}$ captured.

Hence, we are able to verify the information from Climeworks and obtain similar results on Climate Change impacts. Further,

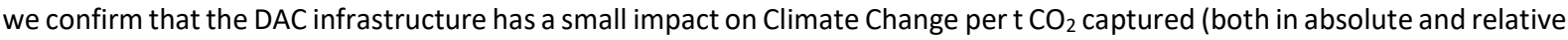
terms compared to other DACCS life-cycle contributions). The main difference between the two approaches originates from the foundation and the process unit. We found lower Climate Change impacts for the foundation in our calculation, since our analysis included a general ecoinvent data proxy with a lifetime of 50 years for the $300 \mathrm{~m}^{2}$ hall, a lifetime which might be optimistic. The contribution of the Process Unit turned out to have a slightly higher Climate Change impact in our analysis, since we estimated the size of the process units based on a figure on the weba.

This simplified infrastructure analysis does not aim to replace original life cycle inventory from Climeworks, since this back of the envelope calculation applies rough assumptions, which differ from reality. However, performing independent calculations based on public information to verify data from industry - which cannot be disclosed in our article - corresponds to best practice in LCA.

Note, again, that we use the detailed life-cycle inventory of Climeworks in our LCA, and that we do not use the life cycle inventory described in this section. Hence, we recommend not utilizing the life cycle inventory as presented in our simplified calculation.

Table 2. Life cycle inventory as used in our back of the envelope calculation for a DAC plant of $4 \mathrm{kt} \mathrm{CO}_{2}$ captured/year and DAC plant of $100 \mathrm{kt}$ $\mathrm{CO}_{2}$ captured/year. The inventories are being shown such that they can be imported with the Brightway2 software easily. An activity is indicated in bold with its corresponding unit and location in column 4 and 6, respectively. Their exchanges can be found under the activity. Activities are separated by a blank row.

\begin{tabular}{|c|c|c|c|c|c|c|}
\hline carbon dioxide capture system_4_kt & & & per t $\mathrm{CO} 2$ captured & & RER & \\
\hline Name & Reference Product & Amount & Unit & Categories & Location & Type \\
\hline collectors_4_kt & & $1.25 \mathrm{E}-05$ & unit & & RER & $\begin{array}{l}\text { technosphe } \\
\text { re }\end{array}$ \\
\hline engineering_4_kt & & $1.25 \mathrm{E}-05$ & unit & & RER & $\begin{array}{l}\text { technosphe } \\
\text { re }\end{array}$ \\
\hline hall_4_kt & & $1.25 \mathrm{E}-05$ & unit & & RER & $\begin{array}{l}\text { technosphe } \\
\text { re }\end{array}$ \\
\hline process unit_4_kt & & $1.25 \mathrm{E}-05$ & unit & & RER & $\begin{array}{l}\text { technosphe } \\
\text { re }\end{array}$ \\
\hline tank_4_kt & & $1.25 \mathrm{E}-05$ & unit & & RER & $\begin{array}{l}\text { technosphe } \\
\text { re }\end{array}$ \\
\hline collectors_4_kt & & & unit & & RER & \\
\hline Name & Reference Product & Amount & Unit & Categories & Location & Type \\
\hline market for passenger car, petrol/natural gas & $\begin{array}{l}\text { passenger car, } \\
\text { petrol/natural gas }\end{array}$ & $7.04 E+04$ & kilogram & & GLO & $\begin{array}{l}\text { technosphe } \\
\text { re }\end{array}$ \\
\hline
\end{tabular}




\begin{tabular}{|c|c|c|c|c|c|c|}
\hline engineering_4_kt & & & unit & & RER & \\
\hline Name & Reference Product & Amount & Unit & Categories & Location & Type \\
\hline Occupation, industrial area & & $8.00 \mathrm{E}+03$ & square meter-year & $\begin{array}{l}\text { natural } \\
\text { resource:lan } \\
\text { d }\end{array}$ & & biosphere \\
\hline $\begin{array}{l}\text { Transformation, from grassland, natural } \\
\text { (non-use) }\end{array}$ & & $4.00 \mathrm{E}+02$ & square meter & $\begin{array}{l}\text { natural } \\
\text { resource:lan } \\
\text { d }\end{array}$ & & biosphere \\
\hline Transformation, to industrial area & & $4.00 \mathrm{E}+02$ & square meter & $\begin{array}{l}\text { natural } \\
\text { resource:lan } \\
\text { d }\end{array}$ & & biosphere \\
\hline market for concrete, normal & concrete, normal & $4.00 \mathrm{E}+02$ & cubic meter & & $\mathrm{CH}$ & $\begin{array}{l}\text { technosphe } \\
\text { re }\end{array}$ \\
\hline market for steel, low-alloyed & steel, low-alloyed & $4.80 \mathrm{E}+04$ & kilogram & & GLO & $\begin{array}{l}\text { technosphe } \\
\text { re }\end{array}$ \\
\hline hall_4_kt & & & unit & & RER & \\
\hline Name & Reference Product & Amount & Unit & Categories & Location & Type \\
\hline market for building, hall, steel construction & $\begin{array}{l}\text { building, hall, steel } \\
\text { construction }\end{array}$ & $1.20 \mathrm{E}+02$ & square meter & & GLO & $\begin{array}{l}\text { technosphe } \\
\text { re }\end{array}$ \\
\hline process unit_4_kt & & & unit & & RER & \\
\hline Name & Reference Product & Amount & Unit & Categories & Location & Type \\
\hline market for passenger car, petrol/natural gas & $\begin{array}{l}\text { passenger car, } \\
\text { petrol/natural gas }\end{array}$ & $4.95 \mathrm{E}+04$ & kilogram & & GLO & $\begin{array}{l}\text { technosphe } \\
\text { re }\end{array}$ \\
\hline tank_4_kt & & & unit & & RER & \\
\hline Name & Reference Product & Amount & Unit & Categories & Location & Type \\
\hline market for steel, low-alloyed & steel, low-alloyed & $3.40 \mathrm{E}+04$ & kilogram & & GLO & $\begin{array}{l}\text { technosphe } \\
\text { re }\end{array}$ \\
\hline carbon dioxide capture system_100_kt & & & per t CO2 captured & & RER & \\
\hline Name & Reference Product & Amount & Unit & Categories & Location & Type \\
\hline collectors_100_kt & & $5.00 \mathrm{E}-07$ & unit & & RER & $\begin{array}{l}\text { technosphe } \\
\text { re }\end{array}$ \\
\hline engineering_100_kt & & $5.00 \mathrm{E}-07$ & unit & & RER & $\begin{array}{l}\text { technosphe } \\
\text { re }\end{array}$ \\
\hline hall_100_kt & & $5.00 \mathrm{E}-07$ & unit & & RER & $\begin{array}{l}\text { technosphe } \\
\text { re }\end{array}$ \\
\hline process unit_100_kt & & $5.00 \mathrm{E}-07$ & unit & & RER & $\begin{array}{l}\text { technosphe } \\
\text { re }\end{array}$ \\
\hline tank_100_kt & & $5.00 \mathrm{E}-07$ & unit & & RER & $\begin{array}{l}\text { technosphe } \\
\text { re }\end{array}$ \\
\hline collectors_100_kt & & & unit & & RER & \\
\hline Name & Reference Product & Amount & Unit & Categories & Location & Type \\
\hline market for passenger car, petrol/natural gas & $\begin{array}{l}\text { passenger car, } \\
\text { petrol/natural gas }\end{array}$ & $8.80 \mathrm{E}+05$ & kilogram & & GLO & $\begin{array}{l}\text { technosphe } \\
\text { re }\end{array}$ \\
\hline engineering_100_kt & & & unit & & RER & \\
\hline Name & Reference Product & Amount & Unit & Categories & Location & Type \\
\hline Occupation, industrial area & & $1.00 \mathrm{E}+05$ & square meter-year & $\begin{array}{l}\text { natural } \\
\text { resource:lan } \\
\text { d }\end{array}$ & & biosphere \\
\hline $\begin{array}{l}\text { Transformation, from grassland, natural } \\
\text { (non-use) }\end{array}$ & & $5.00 \mathrm{E}+03$ & square meter & $\begin{array}{l}\text { natural } \\
\text { resource:lan } \\
\text { d } \\
\end{array}$ & & biosphere \\
\hline Transformation, to industrial area & & $5.00 \mathrm{E}+03$ & square meter & $\begin{array}{l}\text { natural } \\
\text { resource:lan } \\
\text { d }\end{array}$ & & biosphere \\
\hline market for concrete, normal & concrete, normal & $5.00 \mathrm{E}+03$ & cubic meter & & $\mathrm{CH}$ & $\begin{array}{l}\text { technosphe } \\
\text { re }\end{array}$ \\
\hline
\end{tabular}




\begin{tabular}{|c|c|c|c|c|c|c|}
\hline market for steel, low-alloyed & steel, low-alloyed & $6.00 \mathrm{E}+05$ & kilogram & & GLO & $\begin{array}{l}\text { technosphe } \\
\text { re }\end{array}$ \\
\hline hall_100_kt & & & unit & & RER & \\
\hline Name & Reference Product & Amount & Unit & Categories & Location & Type \\
\hline market for building, hall, steel construction & $\begin{array}{l}\text { building, hall, steel } \\
\text { construction }\end{array}$ & $1.50 \mathrm{E}+03$ & square meter & & GLO & $\begin{array}{l}\text { technosphe } \\
\text { re }\end{array}$ \\
\hline process unit_100_kt & & & unit & & RER & \\
\hline Name & Reference Product & Amount & Unit & Categories & Location & Type \\
\hline market for passenger car, petrol/natural gas & $\begin{array}{l}\text { passenger car, } \\
\text { petrol/natural gas }\end{array}$ & $6.11 \mathrm{E}+05$ & kilogram & & GLO & $\begin{array}{l}\text { technosphe } \\
\text { re }\end{array}$ \\
\hline tank_100_kt & & & unit & & RER & \\
\hline Name & Reference Product & Amount & Unit & Categories & Location & Type \\
\hline market for steel, low-alloyed & steel, low-alloyed & $3.81 E+05$ & kilogram & & GLO & $\begin{array}{l}\text { technosphe } \\
\text { re }\end{array}$ \\
\hline
\end{tabular}




\section{S2. Selection of locations for DACCS system configurations}

We consider DACCS installation in eight different countries with corresponding inventory data in our main analysis (see Figure 2 in the main article). This section explains the selection of these countries. First, Iceland is included since the second DACCS unit (4 kt CO 2 captured/year) of Climeworks will be installed in Hellisheiði (Iceland) in 2021. Second, Norway is included since it presents a nearly optimal situation: Norway has clean grid electricity and has $\mathrm{CO}_{2}$ storage potentials nearby (Anthonsen et al., 2013). Further, Switzerland is considered as a region with longer transportation distances to $\mathrm{CO}_{2}$ storage facilities (assumed to be $1500 \mathrm{~km}$ ), and since a DAC unit of Climeworks is operating in Hinwil (Switzerland) with a relatively clean electricity mix and waste heat.

The autonomous DACCS configurations supplied with solar energy were limited to sites in five countries - Chile (CL), Greece (GR), Jordan (JO), Mexico (MX) and Spain (ES) - due to limited data availability for the Fresnel heat collectors. According to the manufacturer of these heat collectors, the system design and the corresponding inventory data are very specific for each location and should not be used for other locations applying simple scaling factors based on annual solar irradiation. These five countries are also included for the grid-coupled alternatives; hence, we also consider countries with $\mathrm{CO}_{2}$ intensive grid electricity mixes (e.g. Greece and Mexico). Autonomous energy systems with solar heat and electricity require a high amount of annual solar irradiation for solar energy production, preferably with a Direct Normal Irradiation (DNI) of more than 2000 $\mathrm{kWh} / \mathrm{m}^{2}$ year, especially for the concentrated thermal solar heat collectors (Kurup et al., 2019). Hence, we present a map (see Figure C) herein to explore other promising locations - with a DNI of more than $2000 \mathrm{kWh} / \mathrm{m}^{2}$ year - for the installation of the autonomous energy system configurations. Figure $\mathrm{C}$ reveals that (especially) northern Mexico, south-west USA, middle Chile, northern Africa, south-west Africa, the Middle East and Australia are potentially promising locations for our proposed autonomous DACCS systems in terms of DNI, although a case-specific assessment is needed to determine their overall (environmental) performance.

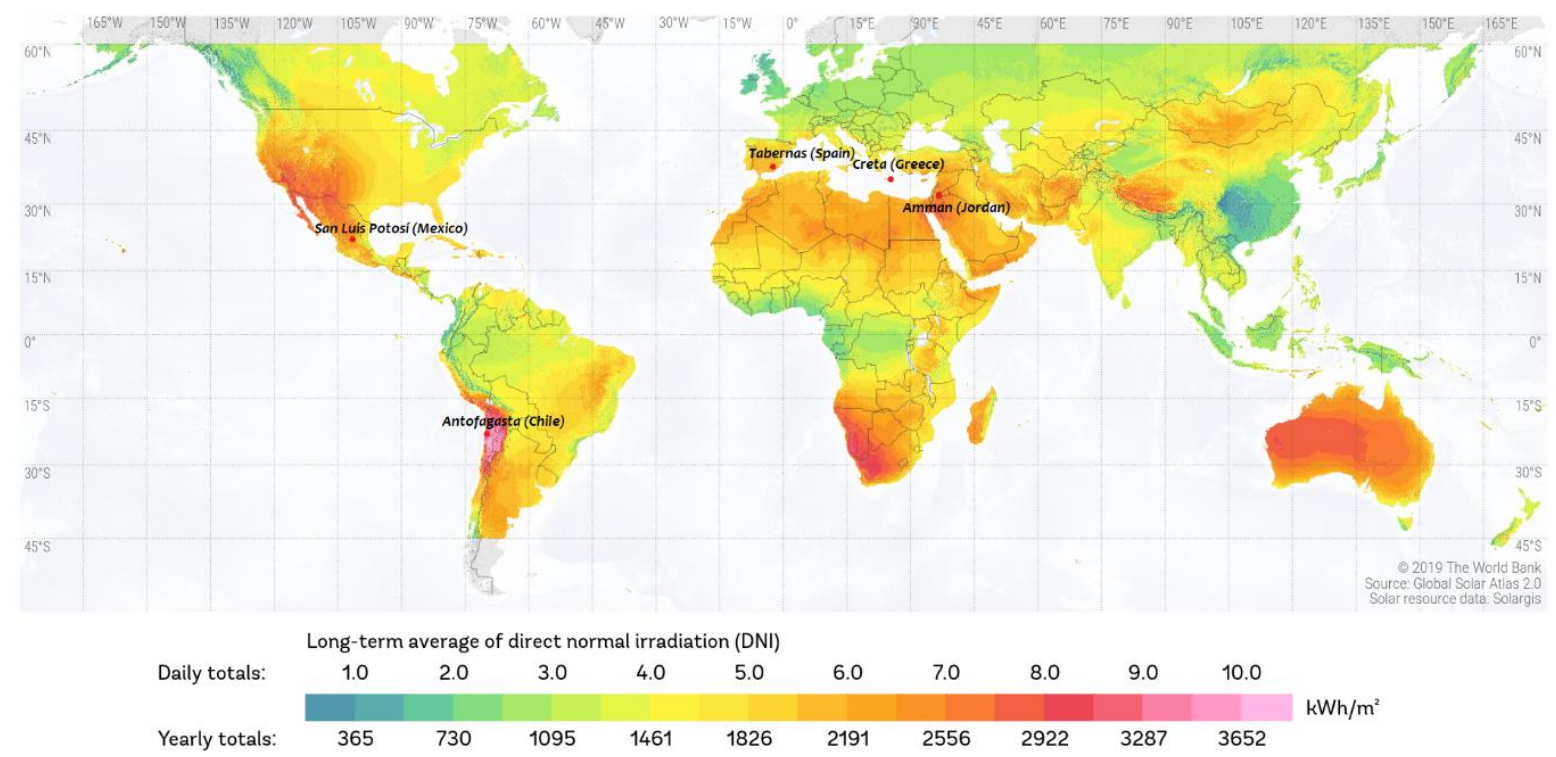

Figure C. Geographical distribution of average direct normal irradiation (DNI). The map presents promising locations for autonomous DACCS energy systems with solar energy, i.e. with a DNI of more than $2000 \mathrm{kWh} / \mathrm{m}^{2}$ year. This solar map is obtained from the "Global Solar Atlas 2.0", a free, web-based application is developed and operated by the company Solargis s.r.o. on behalf of the World Bank Group, utilizing Solargis data, with funding provided by the Energy Sector Management Assistance Program (ESMAP). For additional information: https://globalsolaratlas.info. Copyright: (C) 2019 The World Bank, Source: Global Solar Atlas 2.0, Solar resource data: Solargis., under the CC BY 4.0 license (Solargis, 2020). We added locations to this map as used in our LCA with a red pointer and their name. 


\section{S3. Overview table of energy system layouts}

Table 3 presents the sizing of different system layouts. Note that Autonomous (Fresnel $+P V$ ) has a bigger battery energy storage system and requires more PV electricity generation compared to Waste heat $+P V+$ Battery, since the Autonomous (Fresnel $+P V$ ) layout requires a small additional portion of electricity needed for the system operation of the Fresnel solar collector.

Table 3. Overview of the sizing of system components per alternative.

\begin{tabular}{|c|c|c|c|c|c|c|c|c|c|}
\hline & & $\begin{array}{l}\text { Autonomous } \\
\text { (Fresnel + PV) }\end{array}$ & $\begin{array}{l}\begin{array}{l}\text { Autonomous } \\
\text { (HTHP + PV) }\end{array} \\
\end{array}$ & HTHP + Grid & $\begin{array}{l}\text { Waste heat }+ \\
\text { Grid }\end{array}$ & $\begin{array}{l}\text { Waste heat }+ \\
\text { PV+ Battery }\end{array}$ & Unit & LCI Ref. & $\begin{array}{l}\text { Lifetime } \\
\text { considered }\left(\mathbf{L T}_{\mathrm{x}}=\text { lifetime }\right. \\
\text { component) }\end{array}$ \\
\hline Locations & Country & $\mathrm{CL}, \mathrm{ES}, \mathrm{GR}, \mathrm{JO}, \mathrm{MX}$ & $\begin{array}{l}\mathrm{CL}, \mathrm{ES}, \mathrm{GR}, \mathrm{JO}, \\
\mathrm{MX}\end{array}$ & $\begin{array}{l}\mathrm{CH}, \mathrm{CL}, \mathrm{ES}, \mathrm{GR}, \\
\mathrm{IS}, \\
\mathrm{JO}, \mathrm{MX}, \mathrm{NO}\end{array}$ & $\begin{array}{l}\mathrm{CH}, \mathrm{CL}, \mathrm{ES}, \mathrm{GR}, \\
\mathrm{IS}, \\
\mathrm{JO}, \mathrm{MX}, \mathrm{NO}\end{array}$ & $\mathrm{CL}, \mathrm{ES}, \mathrm{GR}, \mathrm{JO}, \mathrm{MX}$ & Ecoinvent abb. & (Ecoinvent, 2020) & \\
\hline \multirow[b]{2}{*}{ Available DAC capacity } & Size & 100 & 100 & 100 & 100 & 100 & $\begin{array}{l}{\left[\mathrm{kt} \quad \mathrm{CO}_{2}\right.} \\
\text { captured/year] }\end{array}$ & $\begin{array}{l}\text { Factsheet Climeworks (confidential) } \\
\text { and Deutz, Bardow (Deutz and } \\
\text { Bardow, 2021) }\end{array}$ & \\
\hline & Lifetime & 20 & 20 & 20 & 20 & 20 & [years] & $\begin{array}{l}\text { Factsheet Climeworks (confidential) } \\
\text { and Deutz, Bardow (Deutz and } \\
\text { Bardow, 2021) }\end{array}$ & n.a. (system lifetime) \\
\hline \multirow{2}{*}{ Battery pack capacity } & Size & 125 & 221 & 0 & 0 & 120 & $\begin{array}{l}\text { [MWh } \\
\text { electricity storage] }\end{array}$ & (Schmidt et al., 2019) & \\
\hline & Lifetime & 12 & 12 & n.a. & n.a. & 12 & [years] & (Schmidt et al., 2019) & $20 / L T_{x}$ \\
\hline \multirow{2}{*}{ Battery power unit (BoS) } & Size & 63 & 110 & 0 & 0 & 60 & $\begin{array}{l}{[\mathrm{MW}} \\
\text { capacity] }\end{array}$ power & (Schmidt et al., 2019) & \\
\hline & Lifetime & 20 & 20 & n.a. & n.a. & 20 & [years] & (Schmidt et al., 2019) & $20 / L T_{x}$ \\
\hline \multirow[t]{2}{*}{ HTHP } & Size & 0 & 17 & 17 & 0 & 0 & {$[\mathrm{MW}]$} & $\begin{array}{l}\text { Personal communication MAN } \\
\text { Energy } \\
\text { Solutions, (ecoinvent, 2020) }\end{array}$ & \\
\hline & Lifetime & n.a. & 20 & 20 & n.a. & n.a. & [years] & (Ecoinvent, 2020) & $20 / L T_{x}$ \\
\hline \multirow[b]{2}{*}{ Heat storage } & Size & $\sim 210$ & 0 & 0 & 0 & 0 & $\begin{array}{l}\text { [MWh heat } \\
\text { storage] }\end{array}$ & $\begin{array}{l}\text { Personal communication Industrial } \\
\text { Solar }\end{array}$ & \\
\hline & Lifetime & 25 & n.a. & n.a. & n.a. & n.a. & [years] & $\begin{array}{l}\text { Personal communication Industrial } \\
\text { Solar }\end{array}$ & $20 / L T_{x}$ \\
\hline \multirow[b]{2}{*}{ Fresnel } & Size & $\sim 77-108$ & 0 & 0 & 0 & 0 & $\begin{array}{l}{\left[\mathrm{MW}_{\mathrm{p}} \text { heat }\right]} \\
\text { Under reference } \\
\text { conditions }\end{array}$ & $\begin{array}{l}\text { Personal communication Industrial } \\
\text { Solar }\end{array}$ & \\
\hline & Lifetime & 25 & n.a. & n.a. & n.a. & n.a. & [years] & $\begin{array}{l}\text { Personal communication Industrial } \\
\text { Solar }\end{array}$ & n.a., Already considered in FU \\
\hline \multirow[t]{2}{*}{ PV system } & Size & $\sim 37-75$ & $\sim 67-132$ & 0 & 0 & $\sim 36-72$ & {$\left[\mathrm{MW}_{\mathrm{p}}\right]$} & (Ecoinvent, 2020) & \\
\hline & Lifetime & 30 & 30 & n.a. & n.a. & 30 & [years] & (Ecoinvent, 2020) & n.a., Already considered in FU \\
\hline
\end{tabular}




\section{S4. Life cycle inventory: fresnel solar collector}

The $\mathrm{LCl}$ of the fresnel solar collector is based on personal communication with Industrial Solar (Industrial Solar, 2021). This system is operated in Jordan, while performance data for all other locations are based on calculations provided by Industrial Solar. The fresnel construction is largely made of low-alloyed steel and (for a smaller part) of stainless steel and aluminium. Industrial Solar offers commercial solar heat systems, such as the fresnel solar collector LF-11 (Industrial Solar, 2021). Fresnel solar plants use reflective mirrors (made of glass) to concentrate solar irradiation on a solar collector. Water is pumped through the solar collector and is partly evaporated due to the concentration of solar irradiation.

Next, the resulting steam is stored as latent heat in a steam drum reservoir (Industrial Solar, 2021). We scale the heat storage tank, made of low-alloyed steel, to be able to store the amount of steam generated within 12 hours, since the fresnel plant only produces solar-based heat during the day. The fresnel plant is produced in Germany by Industrial Solar. Hence, transportation distances to other countries use Freiburg (Germany) as reference point and include freight transportation by lorries and ships. The latter transportation mode is only used when it is more efficient to reach a destination by ship. Further, we include business trips needed for the acquisition, negotiation, installation, trouble shooting and maintenance of the fresnel plant. Dismantling of the fresnel plant after the system lifetime is considered, with generic recycling, incineration or disposal activities from the ecoinvent database. A system lifetime of 25 years has been assumed. The efficiency of the fresnel plant is obtained from modelling work of Industrial Solar. It varies between $40-47 \%$, mainly influenced by - but not linearly linked to - the incoming direct normal irradiance. The functional unit used in the fresnel LCA is $1 \mathrm{MJ}$ of heat delivered, to be subsequently consumed in the $\mathrm{CO}_{2}$ capture process of the DAC plant.

The operation of the fresnel solar collector requires a small amount of electricity. Since Fresnel heat is used in stand-alone systems with a battery, the electricity consumption is compensated with a safety factor (divided by $89 \%$, i.e. the roundtrip efficiency of the battery) to include potential electricity losses during a battery cycle (the battery life cycle inventory is attributed to the DACCS configuration). A small amount of electricity is required for the fresnel heat collector and is assumed to be provided by PV electricity on rooftops representing installation on existing infrastructures, since the electricity requirement is very small. The total business trips are estimated on 51 trips.

Table 4. Life cycle inventory of the fresnel solar collector. The inventories are being shown such that they can be imported with the Brightway2 software easily. An activity is indicated in bold with its corresponding unit and location in column 4 and 6, respectively. Their exchanges can be found under the activity. Activities are separated by a blank row.

\begin{tabular}{|c|c|c|c|c|c|c|}
\hline collector construction, Fresnel plant, Jordan & & & unit & & jO & \\
\hline Name & Reference Product & $\begin{array}{l}\text { Amou } \\
\text { nt }\end{array}$ & Unit & Categories & $\begin{array}{l}\text { Loca } \\
\text { tion }\end{array}$ & Type \\
\hline Occupation, arable land, unspecified use & & $\begin{array}{r}3.00 \mathrm{E} \\
+04\end{array}$ & $\begin{array}{l}\text { square } \\
\text { meter-year }\end{array}$ & $\begin{array}{l}\text { natural } \\
\text { resource:land }\end{array}$ & & $\begin{array}{l}\text { biosphe } \\
\text { re }\end{array}$ \\
\hline Occupation, urban, green area & & $\begin{array}{r}1.75 \mathrm{E} \\
+04\end{array}$ & $\begin{array}{l}\text { square } \\
\text { meter-year }\end{array}$ & $\begin{array}{l}\text { natural } \\
\text { resource:land }\end{array}$ & & $\begin{array}{l}\text { biosphe } \\
\text { re }\end{array}$ \\
\hline Occupation, urban/industrial fallow (non-use) & & $\begin{array}{r}2.50 \mathrm{E} \\
+03\end{array}$ & $\begin{array}{l}\text { square } \\
\text { meter-year }\end{array}$ & $\begin{array}{l}\text { natural } \\
\text { resource:land }\end{array}$ & & $\begin{array}{l}\text { biosphe } \\
\text { re }\end{array}$ \\
\hline Transformation, from grassland, natural (non-use) & & $\begin{array}{r}2.00 \mathrm{E} \\
+03\end{array}$ & $\begin{array}{l}\text { square } \\
\text { meter }\end{array}$ & $\begin{array}{l}\text { natural } \\
\text { resource:land }\end{array}$ & & $\begin{array}{l}\text { biosphe } \\
\text { re }\end{array}$ \\
\hline Transformation, to arable land, unspecified use & & $\begin{array}{r}1.20 \mathrm{E} \\
+03 \\
\end{array}$ & $\begin{array}{l}\text { square } \\
\text { meter }\end{array}$ & $\begin{array}{l}\text { natural } \\
\text { resource:land }\end{array}$ & & $\begin{array}{l}\text { biosphe } \\
\text { re }\end{array}$ \\
\hline Transformation, to urban, green area & & $\begin{array}{r}7.00 \mathrm{E} \\
+02 \\
\end{array}$ & $\begin{array}{l}\text { square } \\
\text { meter }\end{array}$ & $\begin{array}{l}\text { natural } \\
\text { resource:land }\end{array}$ & & $\begin{array}{l}\text { biosphe } \\
\text { re }\end{array}$ \\
\hline Transformation, to urban/industrial fallow (non-use) & & $\begin{array}{r}1.00 \mathrm{E} \\
+02 \\
\end{array}$ & $\begin{array}{l}\text { square } \\
\text { meter }\end{array}$ & $\begin{array}{l}\text { natural } \\
\text { resource:Iand }\end{array}$ & & $\begin{array}{l}\text { biosphe } \\
\text { re }\end{array}$ \\
\hline collector construction, Fresnel plant, Jordan & $\begin{array}{l}\text { collector, Fresnel plant, } \\
\text { Jordan }\end{array}$ & $\begin{array}{r}1.00 \mathrm{E} \\
+00\end{array}$ & unit & & JO & $\begin{array}{l}\text { producti } \\
\text { on }\end{array}$ \\
\hline market for aluminium, wrought alloy & aluminium, wrought alloy & $\begin{array}{r}2.28 \mathrm{E} \\
+03\end{array}$ & kilogram & & GLO & $\begin{array}{l}\text { technos } \\
\text { phere }\end{array}$ \\
\hline market for copper & copper & $\begin{array}{r}2.77 \mathrm{E} \\
+02\end{array}$ & kilogram & & GLO & $\begin{array}{l}\text { technos } \\
\text { phere }\end{array}$ \\
\hline market for polyethylene, high density, granulate & $\begin{array}{l}\text { polyethylene, high density, } \\
\text { granulate }\end{array}$ & $\begin{array}{r}6.98 \mathrm{E} \\
+01 \\
\end{array}$ & kilogram & & GLO & $\begin{array}{l}\text { technos } \\
\text { phere }\end{array}$ \\
\hline market for silver & silver & $\begin{array}{r}1.77 \mathrm{E} \\
+00 \\
\end{array}$ & kilogram & & GLO & $\begin{array}{l}\text { technos } \\
\text { phere }\end{array}$ \\
\hline market for solar glass, low-iron & solar glass, low-iron & $\begin{array}{r}1.33 \mathrm{E} \\
+04\end{array}$ & kilogram & & GLO & $\begin{array}{l}\text { technos } \\
\text { phere }\end{array}$ \\
\hline market for steel, chromium steel $18 / 8$, hot rolled & $\begin{array}{l}\text { steel, chromium steel } 18 / 8 \text {, } \\
\text { hot rolled }\end{array}$ & $\begin{array}{r}2.72 \mathrm{E} \\
+03\end{array}$ & kilogram & & GLO & $\begin{array}{l}\text { technos } \\
\text { phere }\end{array}$ \\
\hline market for steel, low-alloyed, hot rolled & steel, low-alloyed, hot rolled & $\begin{array}{r}2.84 \mathrm{E} \\
+04\end{array}$ & kilogram & & GLO & $\begin{array}{l}\text { technos } \\
\text { phere }\end{array}$ \\
\hline market for tempering, flat glass & tempering, flat glass & $\begin{array}{r}1.33 \mathrm{E} \\
+04\end{array}$ & kilogram & & GLO & $\begin{array}{l}\text { technos } \\
\text { phere }\end{array}$ \\
\hline zinc coating, coils & zinc coat, coils & $\begin{array}{r}1.99 \mathrm{E} \\
+02\end{array}$ & $\begin{array}{l}\text { square } \\
\text { meter }\end{array}$ & & RoW & $\begin{array}{l}\text { technos } \\
\text { phere }\end{array}$ \\
\hline
\end{tabular}




\begin{tabular}{|c|c|c|c|c|c|c|}
\hline $\begin{array}{l}\text { storage unit production, Fresnel plant, Jordan, 12h } \\
\text { storage }\end{array}$ & & & unit & & JO & \\
\hline Name & Reference Product & $\begin{array}{l}\text { Amou } \\
\text { nt }\end{array}$ & Unit & Categories & $\begin{array}{l}\text { Loca } \\
\text { tion }\end{array}$ & Type \\
\hline Occupation, industrial area & & $\begin{array}{r}2.50 \mathrm{E} \\
+04 \\
\end{array}$ & $\begin{array}{l}\text { square } \\
\text { meter-year }\end{array}$ & $\begin{array}{l}\text { natural } \\
\text { resource:land }\end{array}$ & & $\begin{array}{l}\text { biosphe } \\
\text { re }\end{array}$ \\
\hline Transformation, from grassland, natural (non-use) & & $\begin{array}{r}1.00 \mathrm{E} \\
+03 \\
\end{array}$ & $\begin{array}{l}\text { square } \\
\text { meter }\end{array}$ & $\begin{array}{l}\text { natural } \\
\text { resource:land }\end{array}$ & & $\begin{array}{l}\text { biosphe } \\
\text { re }\end{array}$ \\
\hline Transformation, to industrial area & & $\begin{array}{r}1.00 \mathrm{E} \\
+03 \\
\end{array}$ & $\begin{array}{l}\text { square } \\
\text { meter }\end{array}$ & $\begin{array}{l}\text { natural } \\
\text { resource:land }\end{array}$ & & $\begin{array}{l}\text { biosphe } \\
\text { re }\end{array}$ \\
\hline market for scrap steel & scrap steel & $\begin{array}{r}- \\
5.22 \mathrm{E} \\
+05 \\
\end{array}$ & kilogram & & RoW & $\begin{array}{l}\text { technos } \\
\text { phere }\end{array}$ \\
\hline market for steel, low-alloyed, hot rolled & steel, low-alloyed, hot rolled & $\begin{array}{r}8.03 E \\
+05 \\
\end{array}$ & kilogram & & GLO & $\begin{array}{l}\text { technos } \\
\text { phere }\end{array}$ \\
\hline $\begin{array}{l}\text { storage unit production, Fresnel plant, Jordan, } 12 \mathrm{~h} \\
\text { storage }\end{array}$ & $\begin{array}{l}\text { storage unit, Fresnel plant, } \\
\text { Jordan, } 12 \mathrm{~h} \text { storage }\end{array}$ & $\begin{array}{r}1.00 \mathrm{E} \\
+00 \\
\end{array}$ & unit & & JO & $\begin{array}{l}\text { producti } \\
\text { on }\end{array}$ \\
\hline treatment of waste reinforcement steel, recycling & waste reinforcement steel & $\begin{array}{r}- \\
2.81 \mathrm{E} \\
+05 \\
\end{array}$ & kilogram & & RoW & $\begin{array}{l}\text { technos } \\
\text { phere }\end{array}$ \\
\hline transport pipes installation, Fresnel plant, Jordan & & & unit & & JO & \\
\hline Name & Reference Product & $\begin{array}{l}\text { Amou } \\
\text { nt }\end{array}$ & Unit & Categories & $\begin{array}{l}\text { Loca } \\
\text { tion }\end{array}$ & Type \\
\hline market for drawing of pipe, steel & drawing of pipe, steel & $\begin{array}{r}4.44 \mathrm{E} \\
+03 \\
\end{array}$ & kilogram & & GLO & $\begin{array}{l}\text { technos } \\
\text { phere }\end{array}$ \\
\hline market for steel, low-alloyed, hot rolled & steel, low-alloyed, hot rolled & $\begin{array}{r}4.44 \mathrm{E} \\
+03 \\
\end{array}$ & kilogram & & GLO & $\begin{array}{l}\text { technos } \\
\text { phere }\end{array}$ \\
\hline transport pipes installation, Fresnel plant, Jordan & $\begin{array}{l}\text { transport pipes, Fresnel } \\
\text { plant, Jordan }\end{array}$ & $\begin{array}{r}1.00 \mathrm{E} \\
+00 \\
\end{array}$ & unit & & $\mathrm{JO}$ & $\begin{array}{l}\text { producti } \\
\text { on }\end{array}$ \\
\hline end of life, Fresnel solar collector plant, Jordan & & & unit & & JO & \\
\hline ( & Reference Product & $\begin{array}{l}\text { Amou } \\
\text { nt }\end{array}$ & Unit & Categories & $\begin{array}{l}\text { Loca } \\
\text { tion }\end{array}$ & Type \\
\hline end of life, Fresnel solar collector plant, Jordan & $\begin{array}{l}\text { end of life, Fresnel solar } \\
\text { collector plant, Jordan }\end{array}$ & $\begin{array}{r}1.00 \mathrm{E} \\
+00 \\
\end{array}$ & unit & & JO & $\begin{array}{l}\text { producti } \\
\text { on }\end{array}$ \\
\hline market for scrap copper & scrap copper & $\begin{array}{r}2.77 \mathrm{E} \\
+02 \\
\end{array}$ & kilogram & & RoW & $\begin{array}{l}\text { technos } \\
\text { phere }\end{array}$ \\
\hline market for scrap steel & scrap steel & $\begin{array}{r}2.31 \mathrm{E} \\
+04\end{array}$ & kilogram & & RoW & $\begin{array}{l}\text { technos } \\
\text { phere }\end{array}$ \\
\hline market for waste glass & waste glass & $\begin{array}{r}1.33 \mathrm{E} \\
+04 \\
\end{array}$ & kilogram & & RoW & $\begin{array}{l}\text { technos } \\
\text { phere }\end{array}$ \\
\hline market for waste polyethylene & waste polyethylene & $\begin{array}{r}- \\
6.98 \mathrm{E} \\
+01 \\
\end{array}$ & kilogram & & RoW & $\begin{array}{l}\text { technos } \\
\text { phere }\end{array}$ \\
\hline $\begin{array}{l}\text { treatment of aluminium scrap, post-consumer, prepared } \\
\text { for recycling, at remelter }\end{array}$ & aluminium, wrought alloy & $\begin{array}{r}- \\
2.28 \mathrm{E} \\
+03 \\
\end{array}$ & kilogram & & RoW & $\begin{array}{l}\text { technos } \\
\text { phere }\end{array}$ \\
\hline treatment of waste reinforcement steel, recycling & waste reinforcement steel & $\begin{array}{r}- \\
1.25 \mathrm{E} \\
+04 \\
\end{array}$ & kilogram & & RoW & $\begin{array}{l}\text { technos } \\
\text { phere }\end{array}$ \\
\hline $\begin{array}{l}\text { heat production, Fresnel solar collector plant, Amman, } \\
\text { Jordan }\end{array}$ & & & megajoule & & JO & \\
\hline Name & Reference Product & $\begin{array}{l}\text { Amou } \\
\text { nt }\end{array}$ & Unit & Categories & $\begin{array}{l}\text { Loca } \\
\text { tion }\end{array}$ & Type \\
\hline Water, unspecified natural origin & & $\begin{array}{r}4.82 \mathrm{E} \\
-07 \\
\end{array}$ & $\begin{array}{l}\text { cubic } \\
\text { meter }\end{array}$ & $\begin{array}{l}\text { natural } \\
\text { resource:in } \\
\text { water }\end{array}$ & & $\begin{array}{l}\text { biosphe } \\
\text { re }\end{array}$ \\
\hline collector construction, Fresnel plant, Jordan & $\begin{array}{l}\text { collector, Fresnel plant, } \\
\text { Jordan }\end{array}$ & $\begin{array}{r}9.69 \mathrm{E} \\
-09 \\
\end{array}$ & unit & & $\mathrm{JO}$ & $\begin{array}{l}\text { technos } \\
\text { phere }\end{array}$ \\
\hline $\begin{array}{l}\text { electricity production, photovoltaic, } 3 \mathrm{kWp} \text { slanted-roof } \\
\text { installation, multi-Si, panel, mounted }\end{array}$ & electricity, low voltage & $\begin{array}{r}4.76 \mathrm{E} \\
-03 \\
\end{array}$ & $\begin{array}{l}\text { kilowatt } \\
\text { hour }\end{array}$ & & SA & $\begin{array}{l}\text { technos } \\
\text { phere }\end{array}$ \\
\hline end of life, Fresnel solar collector plant, Jordan & $\begin{array}{l}\text { end of life, Fresnel solar } \\
\text { collector plant, Jordan }\end{array}$ & $\begin{array}{r}9.69 \mathrm{E} \\
-09 \\
\end{array}$ & unit & & JO & $\begin{array}{l}\text { technos } \\
\text { phere }\end{array}$ \\
\hline $\begin{array}{l}\text { heat production, Fresnel solar collector plant, Amman, } \\
\text { Jordan }\end{array}$ & $\begin{array}{l}\text { heat, central or small-scale, } \\
\text { Fresnel plant }\end{array}$ & $\begin{array}{r}1.00 \mathrm{E} \\
+00 \\
\end{array}$ & megajoule & & $\mathrm{JO}$ & $\begin{array}{l}\text { producti } \\
\text { on }\end{array}$ \\
\hline transport pipes installation, Fresnel plant, Jordan & $\begin{array}{l}\text { transport pipes, Fresnel } \\
\text { plant, Jordan }\end{array}$ & $\begin{array}{r}9.69 \mathrm{E} \\
-09 \\
\end{array}$ & unit & & JO & $\begin{array}{l}\text { technos } \\
\text { phere }\end{array}$ \\
\hline transport, freight, lorry $>32$ metric ton, EURO 4 & $\begin{array}{l}\text { transport, freight, lorry }>32 \\
\text { metric ton, EURO4 }\end{array}$ & $\begin{array}{r}3.20 \mathrm{E} \\
-04 \\
\end{array}$ & $\begin{array}{l}\text { ton } \\
\text { kilometer }\end{array}$ & & RoW & $\begin{array}{l}\text { technos } \\
\text { phere }\end{array}$ \\
\hline
\end{tabular}




\begin{tabular}{|c|c|c|c|c|c|c|}
\hline transport, freight, lorry >32 metric ton, EURO 6 & $\begin{array}{l}\text { transport, freight, lorry }>32 \\
\text { metric ton, EURO6 }\end{array}$ & $\begin{array}{r}9.16 \mathrm{E} \\
-04 \\
\end{array}$ & $\begin{array}{l}\text { ton } \\
\text { kilometer }\end{array}$ & & RER & $\begin{array}{l}\text { technos } \\
\text { phere }\end{array}$ \\
\hline transport, freight, sea, container ship & $\begin{array}{l}\text { transport, freight, sea, } \\
\text { container ship }\end{array}$ & $\begin{array}{r}6.89 \mathrm{E} \\
-03 \\
\end{array}$ & $\begin{array}{l}\text { ton } \\
\text { kilometer }\end{array}$ & & GLO & $\begin{array}{l}\text { technos } \\
\text { phere }\end{array}$ \\
\hline transport, passengers, passenger aircraft, medium haul & $\begin{array}{l}\text { transport, passengers, } \\
\text { aircraft, medium haul }\end{array}$ & $\begin{array}{r}3.01 \mathrm{E} \\
-03 \\
\end{array}$ & $\begin{array}{l}\text { person } \\
\text { kilometer }\end{array}$ & & GLO & $\begin{array}{l}\text { technos } \\
\text { phere }\end{array}$ \\
\hline $\begin{array}{l}\text { heat production, Fresnel solar collector plant, } \\
\text { Antofagasto, Chile }\end{array}$ & & & megajoule & & $\mathrm{CL}$ & \\
\hline ( & Reference Product & $\begin{array}{l}\text { Amou } \\
\text { nt }\end{array}$ & Unit & Categories & $\begin{array}{l}\text { Loca } \\
\text { tion }\end{array}$ & Type \\
\hline Water, unspecified natural origin & & $\begin{array}{r}4.05 \mathrm{E} \\
-07 \\
\end{array}$ & $\begin{array}{l}\text { cubic } \\
\text { meter }\end{array}$ & $\begin{array}{l}\text { natural } \\
\text { resource:in } \\
\text { water }\end{array}$ & & $\begin{array}{l}\text { biosphe } \\
\text { re }\end{array}$ \\
\hline collector construction, Fresnel plant, Jordan & $\begin{array}{l}\text { collector, Fresnel plant, } \\
\text { Jordan }\end{array}$ & $\begin{array}{r}8.14 \mathrm{E} \\
-09 \\
\end{array}$ & unit & & jO & $\begin{array}{l}\text { technos } \\
\text { phere }\end{array}$ \\
\hline $\begin{array}{l}\text { electricity production, photovoltaic, } 3 \mathrm{kWp} \text { slanted-roof } \\
\text { installation, multi-Si, panel, mounted }\end{array}$ & electricity, low voltage & $\begin{array}{r}4.00 \mathrm{E} \\
-03 \\
\end{array}$ & $\begin{array}{l}\text { kilowatt } \\
\text { hour }\end{array}$ & & $\mathrm{CL}$ & $\begin{array}{l}\text { technos } \\
\text { phere }\end{array}$ \\
\hline end of life, Fresnel solar collector plant, Jordan & $\begin{array}{l}\text { end of life, Fresnel solar } \\
\text { collector plant, Jordan }\end{array}$ & $\begin{array}{r}8.14 \mathrm{E} \\
-09 \\
\end{array}$ & unit & & JO & $\begin{array}{l}\text { technos } \\
\text { phere }\end{array}$ \\
\hline $\begin{array}{l}\text { heat production, Fresnel solar collector plant, } \\
\text { Antofagasto, Chile }\end{array}$ & $\begin{array}{l}\text { heat, central or small-scale, } \\
\text { Fresnel plant }\end{array}$ & $\begin{array}{r}1.00 \mathrm{E} \\
+00 \\
\end{array}$ & megajoule & & $\mathrm{CL}$ & $\begin{array}{l}\text { producti } \\
\text { on }\end{array}$ \\
\hline transport pipes installation, Fresnel plant, Jordan & $\begin{array}{l}\text { transport pipes, Fresnel } \\
\text { plant, Jordan }\end{array}$ & $\begin{array}{r}8.14 \mathrm{E} \\
-09 \\
\end{array}$ & unit & & jO & $\begin{array}{l}\text { technos } \\
\text { phere }\end{array}$ \\
\hline transport, freight, lorry $>32$ metric ton, EURO4 & $\begin{array}{l}\text { transport, freight, lorry }>32 \\
\text { metric ton, EURO4 }\end{array}$ & $\begin{array}{r}1.10 E \\
-03 \\
\end{array}$ & $\begin{array}{l}\text { ton } \\
\text { kilometer }\end{array}$ & & RoW & $\begin{array}{l}\text { technos } \\
\text { phere }\end{array}$ \\
\hline transport, freight, lorry >32 metric ton, EURO 6 & $\begin{array}{l}\text { transport, freight, lorry }>32 \\
\text { metric ton, EURO6 }\end{array}$ & $\begin{array}{r}8.34 \mathrm{E} \\
-04 \\
\end{array}$ & $\begin{array}{l}\text { ton } \\
\text { kilometer }\end{array}$ & & RoW & $\begin{array}{l}\text { technos } \\
\text { phere }\end{array}$ \\
\hline transport, freight, sea, container ship & $\begin{array}{l}\text { transport, freight, sea, } \\
\text { container ship }\end{array}$ & $\begin{array}{r}1.13 \mathrm{E} \\
-02 \\
\end{array}$ & $\begin{array}{l}\text { ton } \\
\text { kilometer }\end{array}$ & & GLO & $\begin{array}{l}\text { technos } \\
\text { phere }\end{array}$ \\
\hline transport, passengers, passenger aircraft, long haul & $\begin{array}{l}\text { transport, passengers, } \\
\text { aircraft, long haul }\end{array}$ & $\begin{array}{r}4.75 \mathrm{E} \\
-03 \\
\end{array}$ & $\begin{array}{l}\text { person } \\
\text { kilometer }\end{array}$ & & GLO & $\begin{array}{l}\text { technos } \\
\text { phere }\end{array}$ \\
\hline $\begin{array}{l}\text { heat production, Fresnel solar collector plant, San Luis } \\
\text { Potoso, Mexico }\end{array}$ & & & megajoule & & $\mathrm{MX}$ & \\
\hline ( & Reference Product & $\begin{array}{l}\text { Amou } \\
\text { nt }\end{array}$ & Unit & Categories & $\begin{array}{l}\text { Loca } \\
\text { tion }\end{array}$ & Type \\
\hline Water, unspecified natural origin & & $\begin{array}{r}5.63 E \\
-07 \\
\end{array}$ & $\begin{array}{l}\text { cubic } \\
\text { meter }\end{array}$ & $\begin{array}{l}\text { natural } \\
\text { resource:in } \\
\text { water } \\
\end{array}$ & & $\begin{array}{l}\text { biosphe } \\
\text { re }\end{array}$ \\
\hline collector construction, Fresnel plant, Jordan & $\begin{array}{l}\text { collector, Fresnel plant, } \\
\text { Jordan }\end{array}$ & $\begin{array}{r}1.13 \mathrm{E} \\
-08 \\
\end{array}$ & unit & & jO & $\begin{array}{l}\text { technos } \\
\text { phere }\end{array}$ \\
\hline $\begin{array}{l}\text { electricity production, photovoltaic, } 3 \mathrm{kWp} \text { slanted-roof } \\
\text { installation, multi-Si, panel, mounted }\end{array}$ & electricity, low voltage & $\begin{array}{r}5.56 \mathrm{E} \\
-03 \\
\end{array}$ & $\begin{array}{l}\text { kilowatt } \\
\text { hour }\end{array}$ & & $\mathrm{MX}$ & $\begin{array}{l}\text { technos } \\
\text { phere }\end{array}$ \\
\hline end of life, Fresnel solar collector plant, Jordan & $\begin{array}{l}\text { end of life, Fresnel solar } \\
\text { collector plant, Jordan }\end{array}$ & $\begin{array}{r}1.13 \mathrm{E} \\
-08 \\
\end{array}$ & unit & & jO & $\begin{array}{l}\text { technos } \\
\text { phere }\end{array}$ \\
\hline $\begin{array}{l}\text { heat production, Fresnel solar collector plant, San Luis } \\
\text { Potoso, Mexico }\end{array}$ & $\begin{array}{l}\text { heat, central or small-scale, } \\
\text { Fresnel plant }\end{array}$ & $\begin{array}{r}1.00 \mathrm{E} \\
+00 \\
\end{array}$ & megajoule & & $\mathrm{MX}$ & $\begin{array}{l}\text { producti } \\
\text { on }\end{array}$ \\
\hline transport pipes installation, Fresnel plant, Jordan & $\begin{array}{l}\text { transport pipes, Fresnel } \\
\text { plant, Jordan }\end{array}$ & $\begin{array}{r}1.13 \mathrm{E} \\
-08 \\
\end{array}$ & unit & & JO & $\begin{array}{l}\text { technos } \\
\text { phere }\end{array}$ \\
\hline transport, freight, lorry $>32$ metric ton, EURO 4 & $\begin{array}{l}\text { transport, freight, lorry }>32 \\
\text { metric ton, EURO4 }\end{array}$ & $\begin{array}{r}5.32 \mathrm{E} \\
-04 \\
\end{array}$ & $\begin{array}{l}\text { ton } \\
\text { kilometer }\end{array}$ & & RoW & $\begin{array}{l}\text { technos } \\
\text { phere }\end{array}$ \\
\hline transport, freight, lorry $>32$ metric ton, EURO 6 & $\begin{array}{l}\text { transport, freight, lorry }>32 \\
\text { metric ton, EURO6 }\end{array}$ & $\begin{array}{r}1.16 \mathrm{E} \\
-03 \\
\end{array}$ & $\begin{array}{l}\text { ton } \\
\text { kilometer }\end{array}$ & & RoW & $\begin{array}{l}\text { technos } \\
\text { phere }\end{array}$ \\
\hline transport, freight, sea, container ship & $\begin{array}{l}\text { transport, freight, sea, } \\
\text { container ship }\end{array}$ & $\begin{array}{r}1.07 \mathrm{E} \\
-02 \\
\end{array}$ & $\begin{array}{l}\text { ton } \\
\text { kilometer }\end{array}$ & & GLO & $\begin{array}{l}\text { technos } \\
\text { phere }\end{array}$ \\
\hline transport, passengers, passenger aircraft, long haul & $\begin{array}{l}\text { transport, passengers, } \\
\text { aircraft, long haul }\end{array}$ & $\begin{array}{r}5.55 \mathrm{E} \\
-03 \\
\end{array}$ & $\begin{array}{l}\text { person } \\
\text { kilometer }\end{array}$ & & GLO & $\begin{array}{l}\text { technos } \\
\text { phere }\end{array}$ \\
\hline $\begin{array}{l}\text { heat production, Fresnel solar collector plant, } \\
\text { Tabernas, Spain }\end{array}$ & & & megajoule & & ES & \\
\hline Name & Reference Product & $\begin{array}{l}\text { Amou } \\
\text { nt }\end{array}$ & Unit & Categories & $\begin{array}{l}\text { Loca } \\
\text { tion }\end{array}$ & Type \\
\hline Water, unspecified natural origin & & $\begin{array}{r}5.48 \mathrm{E} \\
-07 \\
\end{array}$ & $\begin{array}{l}\text { cubic } \\
\text { meter }\end{array}$ & $\begin{array}{l}\text { natural } \\
\text { resource:in } \\
\text { water } \\
\end{array}$ & & $\begin{array}{l}\text { biosphe } \\
\text { re }\end{array}$ \\
\hline collector construction, Fresnel plant, Jordan & $\begin{array}{l}\text { collector, Fresnel plant, } \\
\text { Jordan }\end{array}$ & $\begin{array}{r}1.10 \mathrm{E} \\
-08 \\
\end{array}$ & unit & & jO & $\begin{array}{l}\text { technos } \\
\text { phere }\end{array}$ \\
\hline $\begin{array}{l}\text { electricity production, photovoltaic, } 3 \mathrm{kWp} \text { slanted-roof } \\
\text { installation, multi-Si, panel, mounted }\end{array}$ & electricity, low voltage & $\begin{array}{r}5.41 \mathrm{E} \\
-03 \\
\end{array}$ & $\begin{array}{l}\text { kilowatt } \\
\text { hour }\end{array}$ & & ES & $\begin{array}{l}\text { technos } \\
\text { phere }\end{array}$ \\
\hline end of life, Fresnel solar collector plant, Jordan & $\begin{array}{l}\text { end of life, Fresnel solar } \\
\text { collector plant, Jordan }\end{array}$ & $\begin{array}{r}1.10 \mathrm{E} \\
-08 \\
\end{array}$ & unit & & JO & $\begin{array}{l}\text { technos } \\
\text { phere }\end{array}$ \\
\hline $\begin{array}{l}\text { heat production, Fresnel solar collector plant, Tabernas, } \\
\text { Spain }\end{array}$ & $\begin{array}{l}\text { heat, central or small-scale, } \\
\text { Fresnel plant }\end{array}$ & $\begin{array}{r}1.00 \mathrm{E} \\
+00 \\
\end{array}$ & megajoule & & ES & $\begin{array}{l}\text { producti } \\
\text { on }\end{array}$ \\
\hline transport pipes installation, Fresnel plant, Jordan & $\begin{array}{l}\text { transport pipes, Fresnel } \\
\text { plant, Jordan }\end{array}$ & $\begin{array}{r}1.10 \mathrm{E} \\
-08\end{array}$ & unit & & JO & $\begin{array}{l}\text { technos } \\
\text { phere }\end{array}$ \\
\hline
\end{tabular}




\begin{tabular}{|c|c|c|c|c|c|c|}
\hline transport, freight, lorry $>32$ metric ton, EURO 4 & $\begin{array}{l}\text { transport, freight, lorry }>32 \\
\text { metric ton, EURO4 }\end{array}$ & $\begin{array}{r}0.00 \mathrm{E} \\
+00\end{array}$ & $\begin{array}{l}\text { ton } \\
\text { kilometer }\end{array}$ & & RoW & $\begin{array}{l}\text { technos } \\
\text { phere }\end{array}$ \\
\hline transport, freight, lorry $>32$ metric ton, EURO 6 & $\begin{array}{l}\text { transport, freight, lorry }>32 \\
\text { metric ton, EURO6 }\end{array}$ & $\begin{array}{r}2.38 \mathrm{E} \\
-03\end{array}$ & $\begin{array}{l}\text { ton } \\
\text { kilometer }\end{array}$ & & RER & $\begin{array}{l}\text { technos } \\
\text { phere }\end{array}$ \\
\hline transport, freight, sea, container ship & $\begin{array}{l}\text { transport, freight, sea, } \\
\text { container ship }\end{array}$ & $\begin{array}{r}0.00 \mathrm{E} \\
+00 \\
\end{array}$ & $\begin{array}{l}\text { ton } \\
\text { kilometer }\end{array}$ & & GLO & $\begin{array}{l}\text { technos } \\
\text { phere }\end{array}$ \\
\hline transport, passengers, passenger aircraft, medium haul & $\begin{array}{l}\text { transport, passengers, } \\
\text { aircraft, medium haul }\end{array}$ & $\begin{array}{r}9.77 \mathrm{E} \\
-04 \\
\end{array}$ & $\begin{array}{l}\text { person } \\
\text { kilometer }\end{array}$ & & GLO & $\begin{array}{l}\text { technos } \\
\text { phere }\end{array}$ \\
\hline $\begin{array}{l}\text { heat production, Fresnel solar collector plant, Creta, } \\
\text { Greece }\end{array}$ & & & megajoule & & GR & \\
\hline Name & Reference Product & $\begin{array}{l}\text { Amou } \\
\text { nt }\end{array}$ & Unit & Categories & $\begin{array}{l}\text { Loca } \\
\text { tion }\end{array}$ & Type \\
\hline Water, unspecified natural origin & & $\begin{array}{r}5.34 \mathrm{E} \\
-07 \\
\end{array}$ & $\begin{array}{l}\text { cubic } \\
\text { meter }\end{array}$ & $\begin{array}{l}\text { natural } \\
\text { resource:in } \\
\text { water }\end{array}$ & & $\begin{array}{l}\text { biosphe } \\
\text { re }\end{array}$ \\
\hline collector construction, Fresnel plant, Jordan & $\begin{array}{l}\text { collector, Fresnel plant, } \\
\text { Jordan }\end{array}$ & $\begin{array}{r}1.07 \mathrm{E} \\
-08 \\
\end{array}$ & unit & & JO & $\begin{array}{l}\text { technos } \\
\text { phere }\end{array}$ \\
\hline $\begin{array}{l}\text { electricity production, photovoltaic, } 3 \mathrm{kWp} \text { slanted-roof } \\
\text { installation, multi-Si, panel, mounted }\end{array}$ & electricity, low voltage & $\begin{array}{r}5.27 \mathrm{E} \\
-03 \\
\end{array}$ & $\begin{array}{l}\text { kilowatt } \\
\text { hour }\end{array}$ & & GR & $\begin{array}{l}\text { technos } \\
\text { phere }\end{array}$ \\
\hline end of life, Fresnel solar collector plant, Jordan & $\begin{array}{l}\text { end of life, Fresnel solar } \\
\text { collector plant, Jordan }\end{array}$ & $\begin{array}{r}1.07 E \\
-08\end{array}$ & unit & & JO & $\begin{array}{l}\text { technos } \\
\text { phere }\end{array}$ \\
\hline $\begin{array}{l}\text { heat production, Fresnel solar collector plant, Creta, } \\
\text { Greece }\end{array}$ & $\begin{array}{l}\text { heat, central or small-scale, } \\
\text { Fresnel plant }\end{array}$ & $\begin{array}{r}1.00 \mathrm{E} \\
+00 \\
\end{array}$ & megajoule & & GR & $\begin{array}{l}\text { producti } \\
\text { on }\end{array}$ \\
\hline transport pipes installation, Fresnel plant, Jordan & $\begin{array}{l}\text { transport pipes, Fresnel } \\
\text { plant, Jordan }\end{array}$ & $\begin{array}{r}1.07 E \\
-08 \\
\end{array}$ & unit & & JO & $\begin{array}{l}\text { technos } \\
\text { phere }\end{array}$ \\
\hline transport, freight, lorry $>32$ metric ton, EURO4 & $\begin{array}{l}\text { transport, freight, lorry }>32 \\
\text { metric ton, EURO4 }\end{array}$ & $\begin{array}{r}0.00 \mathrm{E} \\
+00 \\
\end{array}$ & $\begin{array}{l}\text { ton } \\
\text { kilometer }\end{array}$ & & RoW & $\begin{array}{l}\text { technos } \\
\text { phere }\end{array}$ \\
\hline transport, freight, lorry $>32$ metric ton, EURO 6 & $\begin{array}{l}\text { transport, freight, lorry }>32 \\
\text { metric ton, EURO6 }\end{array}$ & $\begin{array}{r}1.10 \mathrm{E} \\
-03 \\
\end{array}$ & $\begin{array}{l}\text { ton } \\
\text { kilometer }\end{array}$ & & RER & $\begin{array}{l}\text { technos } \\
\text { phere }\end{array}$ \\
\hline transport, freight, sea, container ship & $\begin{array}{l}\text { transport, freight, sea, } \\
\text { container ship }\end{array}$ & $\begin{array}{r}5.72 \mathrm{E} \\
-03 \\
\end{array}$ & $\begin{array}{l}\text { ton } \\
\text { kilometer }\end{array}$ & & GLO & $\begin{array}{l}\text { technos } \\
\text { phere }\end{array}$ \\
\hline transport, passengers, passenger aircraft, medium haul & $\begin{array}{l}\text { transport, passengers, } \\
\text { aircraft, medium haul }\end{array}$ & $\begin{array}{r}1.18 \mathrm{E} \\
-03\end{array}$ & $\begin{array}{l}\text { person } \\
\text { kilometer }\end{array}$ & & GLO & $\begin{array}{l}\text { technos } \\
\text { phere }\end{array}$ \\
\hline
\end{tabular}




\section{S5. LCI - DACCS alternatives and additional information}

The life cycle inventories of all DACCS alternatives and some additional explanation about the LCl are provided in this section.

\section{DAC plant}

The DAC unit as such is the same for all configurations. Specific production and operation data of the DAC plant is based on industrial information provided by Climeworks (Zürich, Switzerland) and cannot be disclosed. However, in order to verify this information, we independently estimate the material requirements for the construction of the DAC unit based on public information. This verification, supplemented by a contribution analysis of life-cycle GHG emissions from the DAC unit construction broken down into contributions by main components is presented in Note S1 of this SI within this supplementary information (SI). We demonstrate similar life-cycle GHG emission resulting from our simplified, selfgenerated life cycle inventory and Climeworks' life cycle inventory. Furthermore, as our LCA results will show, the DAC infrastructure exhibits a small contribution to overall life-cycle GHG emissions and other environmental burdens per ton of gross $\mathrm{CO}_{2}$ removal, which implies that the DAC infrastructure is less important - in terms of environmental impacts compared to other processes within our system boundaries. Due to our independent verification and the comparatively low importance regarding LCA results, we consider using the detailed material inventory provided by Climeworks as legitimate, despite the fact that it cannot be disclosed.

We differentiate between two DAC units: a today's state-of-the art unit representing Climeworks' current technology (4 kt $\mathrm{CO}_{2}$ captured per year), and a future design representing an upscale of their current standard DAC plant to capture $100 \mathrm{kt}$ $\mathrm{CO}_{2}$ per year. These two units mainly differ in terms of material intensity for construction and adsorbent consumption for $\mathrm{CO}_{2}$ capture. Energy requirements for $\mathrm{CO}_{2}$ capture are, however, identical for the two DAC units: $500 \mathrm{kWh}$ per ton $\mathrm{CO}_{2}$ captured for electricity (without electricity consumption for $\mathrm{CO}_{2}$ compression) and $1500 \mathrm{kWh}$ per ton $\mathrm{CO}_{2}$ captured for heat (at around $100^{\circ} \mathrm{C}$ ) (Deutz and Bardow, 2021). For comparison, a recent study of (Hanna et al., 2021) used an energy consumption for DAC of $444 \mathrm{kWh}$ electricity per ton $\mathrm{CO}_{2}$ captured and $1333 \mathrm{kWh}$ heat per ton $\mathrm{CO}_{2}$ captured. As previous DACCS studies indicate that the energy consumption has a substantial influence on LCA results (de Jonge et al., 2019; Deutz and Bardow, 2021), we present a detailed analysis of different energy sources used for $\mathrm{CO}_{2}$ capture, and consider a reduction of electricity consumption to examine the effect on the climate change impacts.

The analysis in the main body of this article represents the upscaled near-future DAC unit, since we expect this upscaling to take place before any large-scale roll-out. To show the consequences of expected technology developments, LCA results for the current DAC unit are shown for comparison in Note S1 of this SI. In our paper, we analyse a DAC plant with an annual gross carbon capture capacity of $100 \mathrm{kt} \mathrm{CO}_{2}$ and a system lifetime of 20 years (Deutz \& Bardow, 2020). Note 'gross', since GHG emissions from all upstream and downstream activities, generated from the entire DACCS life-cycle, are not included in this figure which inevitably leads to less than $100 \mathrm{kt}$ annual net $\mathrm{CO}_{2}$ removal from the atmosphere.

The LT DAC technology of Climeworks uses a cellulose-based solid sorbent functionalized with amines (Fasihi, Efimova and Breyer, 2019). (Deutz and Bardow, 2021) present an overview and environmental assessment of different sorbents potentially used for the $\mathrm{CO}_{2}$ capture process of Climeworks. They show small environmental impacts in absolute terms associated with adsorbent consumption. Therefore, we consider a generic proxy for the adsorbent, 'market for chemical, organic'. Further, we assume that the production of DAC components and related engineering work is conducted in Switzerland.

\section{Business trips}

We consider environmental impacts of business trips for acquisition, negotiation, installation, trouble shooting and maintenance of the DAC plant by Climeworks engineers. Business flights are estimated based on 100 trips required during the system lifetime of the DAC plant: 40 trips for maintenance, 5 trips for trouble shooting, 45 for installation assistance and 10 trips for acquisition. Further, we assume that the collector, process unit and spare parts are produced in Switzerland and are transported to the DACCS location with freight transport (by ship and lorry).

\section{Dismantling}

Dismantling of the DAC plant is included. All main materials for the collector and process unit (e.g. steel, plastics, copper and aluminium) are assumed to be treated after the system lifetime of 20 years.

\section{Geological storage of $\mathrm{CO}_{2}$}

After the $\mathrm{CO}_{2}$ is captured, the $\mathrm{CO}_{2}$ needs to be compressed from 1 bar to 110 bar by consuming locally available electricity, which is in our alternatives provided by the electricity grid or PV installations. We assume that $\mathrm{CO}_{2}$ is transported with pipelines at 110 bar to the injection wells, due to the high capacity needed for large-scale $\mathrm{CO}_{2}$ capture. For simplicity, 80 bar is assumed as pressure at the pipeline end for each configuration (Volkart, Bauer and Boulet, 2013). Additional compression of $\mathrm{CO}_{2}$ is included when the transportation distance is larger than 200 kilometers to compensate for a pressure drop of $\mathrm{CO}_{2}$ (Volkart, Bauer and Boulet, 2013). We consider $\mathrm{CO}_{2}$ leakage from $\mathrm{CO}_{2}$ transmission pipelines using baseline (i.e. Medium) emission factors according to an IPCC report (Holloway et al., 2006), hence we update the LCl of (Volkart, Bauer and Boulet, 
2013) accordingly. After that, the $\mathrm{CO}_{2}$ is injected into wells - using the country-specific electricity mix - to store the $\mathrm{CO}_{2}$ in suitable geological formations, which is considered to exhibit the highest $\mathrm{CO}_{2}$ storage potential, hence we focus on these (Volkart, Bauer and Boulet, 2013). We use the LCl from (Volkart, Bauer and Boulet, 2013) for the infrastructure requirements for transportation, (re)compression and drilling of wells. We parameterize this inventory to generate location specific environmental impacts of $\mathrm{CO}_{2}$ storage, based on the specific transportation distance for $\mathrm{CO}_{2}$ storage in a country. We assess the feasibility of geological $\mathrm{CO}_{2}$ storage based on a geological storage map developed by the Global CCS Institute (Global CCS Institute, 2011). Based on this map, we estimate transportation distances to potential $\mathrm{CO}_{2}$ injection wells in the same or other countries. We categorize our selected countries into short (100 km for Norway, Iceland and Jordan), moderate (500 km for Greece and Spain) and long distances (1500 km for Chile, Mexico and Switzerland) for pipeline transportation of $\mathrm{CO}_{2}$ to the storage and injection wells. A key advantage of DACCS solutions compared to other CDR options is the location independence of the capture step due to the ubiquitous availability of air as the primary feedstock. Hence, DACCS offers the potential to avoid $\mathrm{CO}_{2}$ transport by building DAC plants at available storage sites. The proposed transport distances can therefore be seen as maximum reasonable suggestions for the corresponding countries. For simplicity, we assume a generic $\mathrm{CO}_{2}$ storage depth of 2000 meters for each country, since (Volkart, Bauer and Boulet, 2013) have shown that this depth hardly affects LCA results. $\mathrm{CO}_{2}$ leakage from injection wells is assumed to be negligible (Alcalde et al., 2018; Kelemen et al., 2019).

The activities 'pipeline, supercritical CO2/km' and 'market for gas turbine, $10 \mathrm{MW}$ electrical' (Volkart, Bauer and Boulet, 2013) were initially based on a mass flow quantified by Wildbolz et al.(Wildbolz, 2007) of $250 \mathrm{~kg} \mathrm{CO}_{2} / \mathrm{s}$. The same applies for the drilling of boreholes 'drilling, deep borehole/m' (injection rate of $125 \mathrm{~kg} / \mathrm{s}$ (Wildbolz, 2007)). For simplicity, we linearly scale these life cycle inventories down to represent our mass flow, by multiplying it with a mass flow ratio (Volkart, Bauer and Boulet, 2013). The latter life cycle inventory is presented in Table 6.

Further, we calculate the electricity requirement for injection (for a storage depth of 2000 meter: $24 \mathrm{kWh} / \mathrm{t} \mathrm{CO}_{2}$ captured) and compression (114 kWh/t CO $\mathrm{CO}_{2}$ captured, with compression units with a lifetime of 10 years) using an equation presented in Hendriks et al. (Hendriks, Chris; Wina, Graus; and Bergen V., 2004). Note that a compensation factor for $\mathrm{CO}_{2}$ compression electricity at the DAC unit - division by $89 \%$, i.e. the roundtrip efficiency of the battery - is applied for electricity requirements in DACCS configurations with battery deployment, to compensate for losses during battery cycles. Full life cycle inventory per system layout is provided in Table 7. The exchange 'Carbon dioxide, fossil' refers to losses of $\mathrm{CO}_{2}$ during transportation in pipelines. Further, detailed LCl of the 'carbon dioxide capture system' and 'end of life, carbon dioxide capture system' activities cannot be provided due to confidential LCl information of Climeworks, although Climeworks expects to publish their $\mathrm{LCl}$ in the near future (a simplified calculation to verify the Climeworks inventory has been provided in Note S1 of this SI).

\section{Sorbent}

Adsorbent consumption is expected to decrease from 7.5 to $3.0 \mathrm{~kg}$ adsorbent per ton $\mathrm{CO}_{2}$ captured, based on Climework's analysis and future targets for sorbent consumption (Deutz and Bardow, 2021). The data proxy 'sorbent, generic' refers to the following dataset:

Table 5. Life cycle inventory of sorbent. The inventories are being shown such that they can be imported with the Brightway2 software easily. An activity is indicated in bold with its corresponding unit and location in column 4 and 6 , respectively.

\begin{tabular}{|c|c|c|c|c|c|c|}
\hline sorbent, generic & & & $\begin{array}{l}\text { kilog } \\
\text { ram }\end{array}$ & & RER & \\
\hline Name & Reference Product & Amount & Unit & Categories & $\begin{array}{l}\text { Loca } \\
\text { tion }\end{array}$ & Type \\
\hline market for chemical, organic & chemical, organic & $1.00 \mathrm{E}+00$ & $\begin{array}{l}\text { kilog } \\
\text { ram }\end{array}$ & & GLO & $\begin{array}{l}\text { technos } \\
\text { phere }\end{array}$ \\
\hline $\begin{array}{l}\text { treatment of spent anion exchange resin from potable } \\
\text { water production, municipal incineration }\end{array}$ & $\begin{array}{l}\text { spent anion exchange resin } \\
\text { from potable water } \\
\text { production }\end{array}$ & $-1.00 E+00$ & $\begin{array}{l}\text { kilog } \\
\text { ram }\end{array}$ & & Row & $\begin{array}{l}\text { technos } \\
\text { phere }\end{array}$ \\
\hline
\end{tabular}

\section{PV electricity}

For PV-coupled system layouts, we assume that PV electricity is produced with large ground mounted PV installations: 'electricity production, photovoltaic, 570kWp open ground installation, multi-Si'. For Chile, Greece and Jordan there is no such country-specific PV electricity data available in the ecoinvent 3.6 database. Hence, we create new activities based on the annual kWh yield per kWp panel installed, which are assumed to be $1906 \mathrm{kWh} / \mathrm{kWp}, 1617 \mathrm{kWh} / \mathrm{kWp}$ and $1884 \mathrm{kWh} / \mathrm{kWp}$ for Chile (Antofagasto), Greece (Creta) and Jordan (Amman), respectively (ESMAP et al., 2020).

Multiple exchanges of 'market for electricity, low voltage' can occur for DACCS configurations, such identical exchanges are required for capture (electricity and heat pump), compression as well as the injection of $\mathrm{CO}_{2}$. Land use for the injection wells is not considered, since it is assumed that their corresponding land use is minor as well as they could be situated in marine areas. 
Table 6. Life cycle inventory for the production of pipelines, drilling of boreholes and the transportation of $\mathrm{CO}_{2} \cdot \mathrm{CO}_{2}$ losses during $\mathrm{CO}_{2}$ with pipelines transportation are considered separately, as described in this Note S5 of the SI. Note that 'pipeline, supercritical CO2/km', 'market for gas turbine, 10MW electrical" and "drilling, deep borehole/ $\mathrm{m}$ ' are initially based on bigger mass flow ratio, hence these are scaled down by making use of a mass flow ratio. The inventories are being shown such that they can be imported with the Brightway2 software easily. An activity is indicated in bold with its corresponding unit and location in column 4 and 6 , respectively. Their exchanges can be found under the activity. Activities are separated by a blank row.

\begin{tabular}{|c|c|c|c|c|c|c|}
\hline pipeline, supercritical $\mathrm{CO} 2 / \mathrm{km}$ & & & kilometer & & RER & \\
\hline Name & Reference Product & $\begin{array}{l}\text { Amou } \\
\text { nt }\end{array}$ & Unit & Categories & Location & Type \\
\hline Occupation, construction site & & $\begin{array}{r}3.33 \mathrm{E} \\
+03 \\
\end{array}$ & $\begin{array}{l}\text { square } \\
\text { meter-year }\end{array}$ & natural resource:land & & $\begin{array}{l}\text { biosphe } \\
\text { re }\end{array}$ \\
\hline Transformation, from forest, unspecified & & $\begin{array}{r}2.00 \mathrm{E} \\
+03 \\
\end{array}$ & $\begin{array}{l}\text { square } \\
\text { meter }\end{array}$ & natural resource:land & & $\begin{array}{l}\text { biosphe } \\
\text { re }\end{array}$ \\
\hline $\begin{array}{l}\text { Transformation, to heterogeneous, } \\
\text { agricultural }\end{array}$ & & $\begin{array}{r}2.00 E \\
+03 \\
\end{array}$ & $\begin{array}{l}\text { square } \\
\text { meter }\end{array}$ & natural resource:land & & $\begin{array}{l}\text { biosphe } \\
\text { re }\end{array}$ \\
\hline Water, unspecified natural origin & & $\begin{array}{r}1.87 \mathrm{E} \\
+02 \\
\end{array}$ & $\begin{array}{l}\text { cubic } \\
\text { meter }\end{array}$ & $\begin{array}{l}\text { natural resource:in } \\
\text { water }\end{array}$ & & $\begin{array}{l}\text { biosphe } \\
\text { re }\end{array}$ \\
\hline drawing of pipe, steel & drawing of pipe, steel & $\begin{array}{r}2.70 \mathrm{E} \\
+05 \\
\end{array}$ & kilogram & Materials/fuels & RER & $\begin{array}{l}\text { technos } \\
\text { phere }\end{array}$ \\
\hline gravel and sand quarry operation & sand & $\begin{array}{r}4.40 \mathrm{E} \\
+06 \\
\end{array}$ & kilogram & Materials/fuels & $\mathrm{CH}$ & $\begin{array}{l}\text { technos } \\
\text { phere }\end{array}$ \\
\hline $\begin{array}{l}\text { market for diesel, burned in building } \\
\text { machine }\end{array}$ & & $\begin{array}{r}3.31 \mathrm{E} \\
+06 \\
\end{array}$ & megajoule & Materials/fuels & GLO & $\begin{array}{l}\text { technos } \\
\text { phere }\end{array}$ \\
\hline market for steel, low-alloyed & & $\begin{array}{r}2.70 E \\
+05 \\
\end{array}$ & kilogram & Materials/fuels & GLO & $\begin{array}{l}\text { technos } \\
\text { phere }\end{array}$ \\
\hline market for stone wool, packed & stone wool, packed & $\begin{array}{r}5.12 \mathrm{E} \\
+03 \\
\end{array}$ & kilogram & Materials/fuels & GLO & $\begin{array}{l}\text { technos } \\
\text { phere }\end{array}$ \\
\hline market for transport, freight train & & $\begin{array}{r}5.51 \mathrm{E} \\
+04 \\
\end{array}$ & $\begin{array}{l}\text { ton } \\
\text { kilometer }\end{array}$ & Materials/fuels & $\begin{array}{l}\text { Europe without } \\
\text { Switzerland }\end{array}$ & $\begin{array}{l}\text { technos } \\
\text { phere }\end{array}$ \\
\hline $\begin{array}{l}\text { market for transport, freight, lorry, } \\
\text { unspecified }\end{array}$ & & $\begin{array}{r}3.15 \mathrm{E} \\
+05 \\
\end{array}$ & $\begin{array}{l}\text { ton } \\
\text { kilometer }\end{array}$ & Materials/fuels & RER & $\begin{array}{l}\text { technos } \\
\text { phere }\end{array}$ \\
\hline pipeline, supercritical $\mathrm{CO} 2 / \mathrm{km}$ & & $\begin{array}{r}1.00 \mathrm{E} \\
+00 \\
\end{array}$ & kilometer & NEEDS_2000:CCS & RER & $\begin{array}{l}\text { producti } \\
\text { on }\end{array}$ \\
\hline transport, helicopter & & $\begin{array}{r}2.60 \mathrm{E} \\
+01 \\
\end{array}$ & hour & Materials/fuels & GLO & $\begin{array}{l}\text { technos } \\
\text { phere }\end{array}$ \\
\hline transport, helicopter, LTO cycle & & $\begin{array}{r}1.04 \mathrm{E} \\
+01 \\
\end{array}$ & unit & Materials/fuels & GLO & $\begin{array}{l}\text { technos } \\
\text { phere }\end{array}$ \\
\hline $\begin{array}{l}\text { treatment of inert waste, inert material } \\
\text { landfill }\end{array}$ & & $\begin{array}{r}- \\
4.40 \mathrm{E} \\
+06 \\
\end{array}$ & kilogram & Waste to treatment & $\mathrm{CH}$ & $\begin{array}{l}\text { technos } \\
\text { phere }\end{array}$ \\
\hline $\begin{array}{l}\text { treatment of scrap steel, inert material } \\
\text { landfill }\end{array}$ & scrap steel & $\begin{array}{r}- \\
1.35 \mathrm{E} \\
+05 \\
\end{array}$ & kilogram & Waste to treatment & $\mathrm{CH}$ & $\begin{array}{l}\text { technos } \\
\text { phere }\end{array}$ \\
\hline $\begin{array}{l}\text { treatment of waste mineral wool, inert } \\
\text { material landfill }\end{array}$ & $\begin{array}{l}\text { waste mineral wool, } \\
\text { for final disposal }\end{array}$ & $\begin{array}{r}- \\
5.12 \mathrm{E} \\
+03 \\
\end{array}$ & kilogram & Waste to treatment & $\mathrm{CH}$ & $\begin{array}{l}\text { technos } \\
\text { phere }\end{array}$ \\
\hline $\begin{array}{l}\text { transport, pipeline, supercritical } \mathrm{CO} \text {, } \\
200 \mathrm{~km} \text { w recompression }\end{array}$ & & & $\begin{array}{l}\text { ton } \\
\text { kilometer }\end{array}$ & & RER & \\
\hline Name & Reference Product & $\begin{array}{l}\text { Amou } \\
\text { nt }\end{array}$ & Unit & Categories & Location & Type \\
\hline market for gas turbine, $10 \mathrm{MW}$ electrical & & $\begin{array}{r}8.04 \mathrm{E}- \\
11 \\
\end{array}$ & unit & Materials/fuels & GLO & $\begin{array}{l}\text { technos } \\
\text { phere }\end{array}$ \\
\hline $\begin{array}{l}\text { market group for electricity, medium } \\
\text { voltage }\end{array}$ & & $\begin{array}{r}3.89 \mathrm{E}- \\
02 \\
\end{array}$ & $\begin{array}{l}\text { kilowatt } \\
\text { hour }\end{array}$ & Materials/fuels & ENTSO-E & $\begin{array}{l}\text { technos } \\
\text { phere }\end{array}$ \\
\hline pipeline, supercritical $\mathrm{CO} 2 / \mathrm{km}$ & & $\begin{array}{r}4.23 \mathrm{E}- \\
09 \\
\end{array}$ & kilometer & Materials/fuels & RER & $\begin{array}{l}\text { technos } \\
\text { phere }\end{array}$ \\
\hline $\begin{array}{l}\text { transport, pipeline, supercritical CO2, } \\
200 \mathrm{~km} \text { w recompression }\end{array}$ & & $\begin{array}{r}1.00 \mathrm{E} \\
+00 \\
\end{array}$ & $\begin{array}{l}\text { ton } \\
\text { kilometer }\end{array}$ & NEEDS_2000:CCS & RER & $\begin{array}{l}\text { producti } \\
\text { on }\end{array}$ \\
\hline $\begin{array}{l}\text { transport, pipeline, supercritical CO2, } \\
200 \mathrm{~km} \text { w/o recompression }\end{array}$ & & & $\begin{array}{l}\text { ton } \\
\text { kilometer }\end{array}$ & & RER & \\
\hline Name & Reference Product & $\begin{array}{l}\text { Amou } \\
\text { nt }\end{array}$ & Unit & Categories & Location & Type \\
\hline pipeline, supercritical $\mathrm{CO} 2 / \mathrm{km}$ & & $\begin{array}{r}4.23 \mathrm{E}- \\
09 \\
\end{array}$ & kilometer & Materials/fuels & RER & $\begin{array}{l}\text { technos } \\
\text { phere }\end{array}$ \\
\hline $\begin{array}{l}\text { transport, pipeline, supercritical CO2, } \\
200 \mathrm{~km} \text { w/o recompression }\end{array}$ & & $\begin{array}{r}1.00 \mathrm{E} \\
+00 \\
\end{array}$ & $\begin{array}{l}\text { ton } \\
\text { kilometer }\end{array}$ & NEEDS_2000:CCS & RER & $\begin{array}{l}\text { producti } \\
\text { on }\end{array}$ \\
\hline drilling, deep borehole/m & & & meter & & RER & \\
\hline Name & Reference Product & $\begin{array}{l}\text { Amou } \\
\text { nt }\end{array}$ & Unit & Categories & Location & Type \\
\hline AOX, Adsorbable Organic Halogen as $\mathrm{Cl}$ & & $\begin{array}{r}4.78 \mathrm{E}- \\
07 \\
\end{array}$ & kilogram & water:surface water & & $\begin{array}{l}\text { biosphe } \\
\text { re }\end{array}$ \\
\hline
\end{tabular}




\begin{tabular}{|c|c|c|c|c|c|c|}
\hline Aluminium & & $\begin{array}{r}6.00 \mathrm{E}- \\
02 \\
\end{array}$ & kilogram & water:surface water & & $\begin{array}{l}\text { biosphe } \\
\text { re }\end{array}$ \\
\hline Arsenic, ion & & $\begin{array}{r}4.20 \mathrm{E}- \\
04\end{array}$ & kilogram & water:surface water & & $\begin{array}{l}\text { biosphe } \\
\text { re }\end{array}$ \\
\hline BOD5, Biological Oxygen Demand & & $\begin{array}{r}3.00 \mathrm{E}- \\
01 \\
\end{array}$ & kilogram & water:surface water & & $\begin{array}{l}\text { biosphe } \\
\text { re }\end{array}$ \\
\hline Barium & & $\begin{array}{r}6.00 \mathrm{E}- \\
03 \\
\end{array}$ & kilogram & water:surface water & & $\begin{array}{l}\text { biosphe } \\
\text { re }\end{array}$ \\
\hline Boron & & $\begin{array}{r}9.00 \mathrm{E}- \\
03\end{array}$ & kilogram & water:surface water & & $\begin{array}{l}\text { biosphe } \\
\text { re }\end{array}$ \\
\hline COD, Chemical Oxygen Demand & & $\begin{array}{r}3.00 \mathrm{E} \\
+00 \\
\end{array}$ & kilogram & water:surface water & & $\begin{array}{l}\text { biosphe } \\
\text { re }\end{array}$ \\
\hline Calcium, ion & & $\begin{array}{r}6.00 \mathrm{E}- \\
01 \\
\end{array}$ & kilogram & water:surface water & & $\begin{array}{l}\text { biosphe } \\
\text { re }\end{array}$ \\
\hline Chloride & & $\begin{array}{r}6.00 \mathrm{E} \\
+00\end{array}$ & kilogram & water:surface water & & $\begin{array}{l}\text { biosphe } \\
\text { re }\end{array}$ \\
\hline Chromium, ion & & $\begin{array}{r}6.00 \mathrm{E}- \\
04 \\
\end{array}$ & kilogram & water:surface water & & $\begin{array}{l}\text { biosphe } \\
\text { re }\end{array}$ \\
\hline DOC, Dissolved Organic Carbon & & $\begin{array}{r}3.00 \mathrm{E}- \\
01 \\
\end{array}$ & kilogram & water:surface water & & $\begin{array}{l}\text { biosphe } \\
\text { re }\end{array}$ \\
\hline Fluoride & & $\begin{array}{r}3.00 \mathrm{E}- \\
03 \\
\end{array}$ & kilogram & water:surface water & & $\begin{array}{l}\text { biosphe } \\
\text { re }\end{array}$ \\
\hline Hydrocarbons, aromatic & & $\begin{array}{r}3.00 \mathrm{E}- \\
03 \\
\end{array}$ & kilogram & water:surface water & & $\begin{array}{l}\text { biosphe } \\
\text { re }\end{array}$ \\
\hline Iron, ion & & $\begin{array}{r}1.80 \mathrm{E}- \\
01 \\
\end{array}$ & kilogram & water:surface water & & $\begin{array}{l}\text { biosphe } \\
\text { re }\end{array}$ \\
\hline Magnesium & & $\begin{array}{r}1.20 \mathrm{E}- \\
01 \\
\end{array}$ & kilogram & water:surface water & & $\begin{array}{l}\text { biosphe } \\
\text { re }\end{array}$ \\
\hline Manganese & & $\begin{array}{r}3.00 \mathrm{E}- \\
03 \\
\end{array}$ & kilogram & water:surface water & & $\begin{array}{l}\text { biosphe } \\
\text { re }\end{array}$ \\
\hline Methane, dichloro-, HCC-30 & & $\begin{array}{r}6.00 \mathrm{E}- \\
02 \\
\end{array}$ & kilogram & water:surface water & & $\begin{array}{l}\text { biosphe } \\
\text { re }\end{array}$ \\
\hline Particulates, $>10$ um & & $\begin{array}{r}1.48 \mathrm{E}- \\
02 \\
\end{array}$ & kilogram & $\begin{array}{l}\text { air:non-urban air or } \\
\text { from high stacks }\end{array}$ & & $\begin{array}{l}\text { biosphe } \\
\text { re }\end{array}$ \\
\hline Phosphorus & & $\begin{array}{r}1.20 \mathrm{E}- \\
03 \\
\end{array}$ & kilogram & water:surface water & & $\begin{array}{l}\text { biosphe } \\
\text { re }\end{array}$ \\
\hline Potassium, ion & & $\begin{array}{r}9.00 \mathrm{E}- \\
01 \\
\end{array}$ & kilogram & water:surface water & & $\begin{array}{l}\text { biosphe } \\
\text { re }\end{array}$ \\
\hline Silicon & & $\begin{array}{r}3.00 \mathrm{E}- \\
02 \\
\end{array}$ & kilogram & water:surface water & & $\begin{array}{l}\text { biosphe } \\
\text { re }\end{array}$ \\
\hline Sodium, ion & & $\begin{array}{r}6.00 \mathrm{E} \\
+00 \\
\end{array}$ & kilogram & water:surface water & & $\begin{array}{l}\text { biosphe } \\
\text { re }\end{array}$ \\
\hline Strontium & & $\begin{array}{r}1.80 \mathrm{E}- \\
02 \\
\end{array}$ & kilogram & water:surface water & & $\begin{array}{l}\text { biosphe } \\
\text { re }\end{array}$ \\
\hline Sulfur & & $\begin{array}{r}1.20 \mathrm{E}- \\
01 \\
\end{array}$ & kilogram & water:surface water & & $\begin{array}{l}\text { biosphe } \\
\text { re }\end{array}$ \\
\hline TOC, Total Organic Carbon & & $\begin{array}{r}3.00 \mathrm{E}- \\
01\end{array}$ & kilogram & water:surface water & & $\begin{array}{l}\text { biosphe } \\
\text { re }\end{array}$ \\
\hline Water, well, in ground & & $\begin{array}{r}3.34 \mathrm{E} \\
+00 \\
\end{array}$ & $\begin{array}{l}\text { cubic } \\
\text { meter }\end{array}$ & $\begin{array}{l}\text { natural resource:in } \\
\text { water }\end{array}$ & & $\begin{array}{l}\text { biosphe } \\
\text { re }\end{array}$ \\
\hline Zinc, ion & & $\begin{array}{r}1.20 \mathrm{E}- \\
03 \\
\end{array}$ & kilogram & water:surface water & & $\begin{array}{l}\text { biosphe } \\
\text { re }\end{array}$ \\
\hline activated bentonite production & activated bentonite & $\begin{array}{r}2.00 \mathrm{E} \\
+01 \\
\end{array}$ & kilogram & Materials/fuels & $\mathrm{DE}$ & $\begin{array}{l}\text { technos } \\
\text { phere }\end{array}$ \\
\hline barite production & barite & $\begin{array}{r}2.70 \mathrm{E} \\
+02\end{array}$ & kilogram & Materials/fuels & RER & $\begin{array}{l}\text { technos } \\
\text { phere }\end{array}$ \\
\hline cement production, Portland & cement, Portland & $\begin{array}{r}2.00 \mathrm{E} \\
+02 \\
\end{array}$ & kilogram & Materials/fuels & $\mathrm{CH}$ & $\begin{array}{l}\text { technos } \\
\text { phere }\end{array}$ \\
\hline $\begin{array}{l}\text { diesel, burned in diesel-electric } \\
\text { generating set, } 18.5 \mathrm{~kW}\end{array}$ & & $\begin{array}{r}6.30 \mathrm{E} \\
+03 \\
\end{array}$ & megajoule & Materials/fuels & GLO & $\begin{array}{l}\text { technos } \\
\text { phere }\end{array}$ \\
\hline drilling, deep borehole/m & & $\begin{array}{r}1.00 \mathrm{E} \\
+00 \\
\end{array}$ & meter & NEEDS_2000:CCS & RER & $\begin{array}{l}\text { producti } \\
\text { on }\end{array}$ \\
\hline lignite mine operation & lignite & $\begin{array}{r}2.00 \mathrm{E}- \\
01 \\
\end{array}$ & kilogram & Materials/fuels & RER & $\begin{array}{l}\text { technos } \\
\text { phere }\end{array}$ \\
\hline market for chemical, organic & & $\begin{array}{r}9.05 \mathrm{E} \\
+00 \\
\end{array}$ & kilogram & Materials/fuels & GLO & $\begin{array}{l}\text { technos } \\
\text { phere }\end{array}$ \\
\hline market for chemicals, inorganic & & $\begin{array}{r}4.22 \mathrm{E} \\
+01 \\
\end{array}$ & kilogram & Materials/fuels & GLO & $\begin{array}{l}\text { technos } \\
\text { phere }\end{array}$ \\
\hline market for lubricating oil & & $\begin{array}{r}6.00 \mathrm{E} \\
+01\end{array}$ & kilogram & Materials/fuels & RER & $\begin{array}{l}\text { technos } \\
\text { phere }\end{array}$ \\
\hline market for steel, low-alloyed & & $\begin{array}{r}2.10 \mathrm{E} \\
+02 \\
\end{array}$ & kilogram & Materials/fuels & GLO & $\begin{array}{l}\text { technos } \\
\text { phere }\end{array}$ \\
\hline market for transport, freight train & & $\begin{array}{r}4.87 \mathrm{E} \\
+02 \\
\end{array}$ & $\begin{array}{l}\text { ton } \\
\text { kilometer }\end{array}$ & Materials/fuels & $\begin{array}{l}\text { Europe without } \\
\text { Switzerland }\end{array}$ & $\begin{array}{l}\text { technos } \\
\text { phere }\end{array}$ \\
\hline $\begin{array}{l}\text { market for transport, freight, lorry, } \\
\text { unspecified }\end{array}$ & & $\begin{array}{r}8.11 \mathrm{E} \\
+01 \\
\end{array}$ & $\begin{array}{l}\text { ton } \\
\text { kilometer }\end{array}$ & Materials/fuels & RER & $\begin{array}{l}\text { technos } \\
\text { phere }\end{array}$ \\
\hline treatment of drilling waste, landfarming & drilling waste & $\begin{array}{r}2.37 \mathrm{E} \\
+02\end{array}$ & kilogram & Waste to treatment & $\mathrm{CH}$ & $\begin{array}{l}\text { technos } \\
\text { phere }\end{array}$ \\
\hline
\end{tabular}




\begin{tabular}{|c|c|c|c|c|c|c|}
\hline $\begin{array}{l}\text { treatment of drilling waste, residual } \\
\text { material landfill }\end{array}$ & drilling waste & $\begin{array}{r}1.58 \mathrm{E} \\
+02\end{array}$ & kilogram & Waste to treatment & $\mathrm{CH}$ & $\begin{array}{l}\text { technos } \\
\text { phere }\end{array}$ \\
\hline $\begin{array}{l}\text { treatment of hazardous waste, hazardous } \\
\text { waste incineration }\end{array}$ & & $\begin{array}{r}5.00 \mathrm{E} \\
+00\end{array}$ & kilogram & Waste to treatment & $\mathrm{CH}$ & $\begin{array}{l}\text { technos } \\
\text { phere }\end{array}$ \\
\hline
\end{tabular}

Table 7. Life cycle inventory of all configurations. The inventories are being shown such that they can be imported with the Brightway2 software easily. An activity is indicated in bold with its corresponding unit and location in column 4 and 6, respectively. Their exchanges can be found under the activity. Activities are separated by a blank row.

\begin{tabular}{|c|c|c|c|c|c|c|}
\hline $\begin{array}{l}\text { carbon dioxide, captured from atmosphere, Waste heat + } \\
\text { Grid, ES }\end{array}$ & & & ton & & ES & \\
\hline Name & Reference Product & $\begin{array}{l}\text { Amo } \\
\text { unt }\end{array}$ & Unit & Categories & $\begin{array}{l}\text { Loca } \\
\text { tion }\end{array}$ & Type \\
\hline Carbon dioxide, fossil & & $\begin{array}{r}7.00 \\
E+00 \\
\end{array}$ & kilogram & $\begin{array}{l}\text { air:urban air } \\
\text { close to ground }\end{array}$ & & $\begin{array}{l}\text { biosph } \\
\text { ere }\end{array}$ \\
\hline carbon dioxide capture system & & $\begin{array}{l}5.00 \\
\mathrm{E}-07 \\
\end{array}$ & unit & & RER & $\begin{array}{l}\text { techno } \\
\text { sphere }\end{array}$ \\
\hline drilling, deep borehole/m & & $\begin{array}{l}3.38 \\
\mathrm{E}-05 \\
\end{array}$ & meter & & RER & $\begin{array}{l}\text { techno } \\
\text { sphere }\end{array}$ \\
\hline end of life, carbon dioxide capture system & & $\begin{array}{l}5.00 \\
\mathrm{E}-07\end{array}$ & unit & & RoW & $\begin{array}{l}\text { techno } \\
\text { sphere }\end{array}$ \\
\hline $\begin{array}{l}\text { heat, from municipal waste incineration to generic market for } \\
\text { heat district or industrial, other than natural gas }\end{array}$ & $\begin{array}{l}\text { heat, district or industrial, } \\
\text { other than natural gas }\end{array}$ & $\begin{array}{r}5.40 \\
\mathrm{E}+03\end{array}$ & $\begin{array}{l}\text { megajoul } \\
\mathrm{e}\end{array}$ & & ES & $\begin{array}{l}\text { techno } \\
\text { sphere }\end{array}$ \\
\hline market for air compressor, screw-type compressor, $300 \mathrm{~kW}$ & $\begin{array}{l}\text { air compressor, screw-type } \\
\text { compressor, } 300 \mathrm{~kW}\end{array}$ & $\begin{array}{l}4.35 \\
\mathrm{E}-06 \\
\end{array}$ & unit & & GLO & $\begin{array}{l}\text { techno } \\
\text { sphere }\end{array}$ \\
\hline market for electricity, low voltage & electricity, low voltage & $\begin{array}{r}5.00 \\
E+02 \\
\end{array}$ & $\begin{array}{l}\text { kilowatt } \\
\text { hour }\end{array}$ & & ES & $\begin{array}{l}\text { techno } \\
\text { sphere }\end{array}$ \\
\hline market for electricity, low voltage & electricity, low voltage & $\begin{array}{r}1.14 \\
E+02 \\
\end{array}$ & $\begin{array}{l}\text { kilowatt } \\
\text { hour }\end{array}$ & & ES & $\begin{array}{l}\text { techno } \\
\text { sphere }\end{array}$ \\
\hline market for electricity, low voltage & electricity, low voltage & $\begin{array}{r}2.42 \\
E+01\end{array}$ & $\begin{array}{l}\text { kilowatt } \\
\text { hour }\end{array}$ & & ES & $\begin{array}{l}\text { techno } \\
\text { sphere }\end{array}$ \\
\hline market for gas turbine, $10 \mathrm{MW}$ electrical & $\begin{array}{l}\text { gas turbine, } 10 \mathrm{MW} \\
\text { electrical }\end{array}$ & $\begin{array}{l}2.54 \\
\mathrm{E}-08\end{array}$ & unit & & GLO & $\begin{array}{l}\text { techno } \\
\text { sphere }\end{array}$ \\
\hline sorbent, generic & & $\begin{array}{r}3.00 \\
E+00\end{array}$ & kilogram & & RER & $\begin{array}{l}\text { techno } \\
\text { sphere }\end{array}$ \\
\hline transport, freight, lorry $>32$ metric ton, EURO 6 & $\begin{array}{l}\text { transport, freight, lorry } \\
>32 \text { metric ton, EURO6 }\end{array}$ & $\begin{array}{l}3.55 \\
\mathrm{E}-01 \\
\end{array}$ & $\begin{array}{l}\text { ton } \\
\text { kilomete } \\
r\end{array}$ & & RoW & $\begin{array}{l}\text { techno } \\
\text { sphere }\end{array}$ \\
\hline transport, freight, sea, container ship & $\begin{array}{l}\text { transport, freight, sea, } \\
\text { container ship }\end{array}$ & $\begin{array}{l}7.04 \\
E-01 \\
\end{array}$ & $\begin{array}{l}\text { ton } \\
\text { kilomete } \\
r\end{array}$ & & GLO & $\begin{array}{l}\text { techno } \\
\text { sphere }\end{array}$ \\
\hline transport, passengers, passenger aircraft, medium haul & $\begin{array}{l}\text { transport, passengers, } \\
\text { aircraft, medium haul }\end{array}$ & $\begin{array}{l}1.58 \\
\mathrm{E}-01 \\
\end{array}$ & $\begin{array}{l}\text { person } \\
\text { kilomete } \\
r\end{array}$ & & GLO & $\begin{array}{l}\text { techno } \\
\text { sphere }\end{array}$ \\
\hline $\begin{array}{l}\text { transport, pipeline, supercritical CO2, } 200 \mathrm{~km} \mathrm{w} \\
\text { recompression }\end{array}$ & & $\begin{array}{r}3.00 \\
E+02 \\
\end{array}$ & $\begin{array}{l}\text { ton } \\
\text { kilomete } \\
r\end{array}$ & & RER & $\begin{array}{l}\text { techno } \\
\text { sphere }\end{array}$ \\
\hline $\begin{array}{l}\text { transport, pipeline, supercritical CO2, } 200 \mathrm{~km} \text { w/o } \\
\text { recompression }\end{array}$ & & $\begin{array}{r}2.00 \\
E+02 \\
\end{array}$ & $\begin{array}{l}\text { ton } \\
\text { kilomete } \\
r\end{array}$ & & RER & $\begin{array}{l}\text { techno } \\
\text { sphere }\end{array}$ \\
\hline $\begin{array}{l}\text { carbon dioxide, captured from atmosphere, Waste heat + } \\
\text { PV + Battery, ES }\end{array}$ & & & ton & & ES & \\
\hline 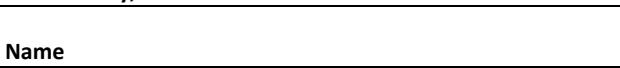 & Reference Product & $\begin{array}{l}\text { Amo } \\
\text { unt }\end{array}$ & Unit & Categories & $\begin{array}{l}\text { Loca } \\
\text { tion }\end{array}$ & Type \\
\hline Battery management system, $\mathrm{kWh}$ & & $\begin{array}{l}9.99 \\
\mathrm{E}-02 \\
\end{array}$ & $\begin{array}{l}\text { kilowatt } \\
\text { hour }\end{array}$ & & GLO & $\begin{array}{l}\text { techno } \\
\text { sphere }\end{array}$ \\
\hline Carbon dioxide, fossil & & $\begin{array}{r}7.00 \\
E+00 \\
\end{array}$ & kilogram & $\begin{array}{l}\text { air:urban air } \\
\text { close to ground }\end{array}$ & & $\begin{array}{l}\text { biosph } \\
\text { ere }\end{array}$ \\
\hline Energy management system, $\mathrm{kWh}$ & & $\begin{array}{l}9.99 \\
\mathrm{E}-02 \\
\end{array}$ & $\begin{array}{l}\text { kilowatt } \\
\text { hour }\end{array}$ & & GLO & $\begin{array}{l}\text { techno } \\
\text { sphere }\end{array}$ \\
\hline Li-ion (NMC) & & $\begin{array}{l}9.99 \\
\mathrm{E}-02 \\
\end{array}$ & $\begin{array}{l}\text { kilowatt } \\
\text { hour }\end{array}$ & & GLO & $\begin{array}{l}\text { techno } \\
\text { sphere }\end{array}$ \\
\hline Power conditioning system, container system & & $\begin{array}{l}3.00 \\
\mathrm{E}-02\end{array}$ & $\mathrm{~kW}$ & & GLO & $\begin{array}{l}\text { techno } \\
\text { sphere }\end{array}$ \\
\hline carbon dioxide capture system & & $\begin{array}{l}5.00 \\
\mathrm{E}-07\end{array}$ & unit & & RER & $\begin{array}{l}\text { techno } \\
\text { sphere }\end{array}$ \\
\hline drilling, deep borehole/m & & $\begin{array}{l}3.38 \\
\mathrm{E}-05 \\
\end{array}$ & meter & & RER & $\begin{array}{l}\text { techno } \\
\text { sphere }\end{array}$ \\
\hline $\begin{array}{l}\text { electricity production, photovoltaic, 570kWp open ground } \\
\text { installation, multi-Si }\end{array}$ & electricity, low voltage & $\begin{array}{r}5.62 \\
E+02 \\
\end{array}$ & $\begin{array}{l}\text { kilowatt } \\
\text { hour }\end{array}$ & & ES & $\begin{array}{l}\text { techno } \\
\text { sphere }\end{array}$ \\
\hline $\begin{array}{l}\text { electricity production, photovoltaic, 570kWp open ground } \\
\text { installation, multi-Si }\end{array}$ & electricity, low voltage & $\begin{array}{r}1.29 \\
\mathrm{E}+02 \\
\end{array}$ & $\begin{array}{l}\text { kilowatt } \\
\text { hour }\end{array}$ & & ES & $\begin{array}{l}\text { techno } \\
\text { sphere }\end{array}$ \\
\hline end of life, carbon dioxide capture system & & $\begin{array}{l}5.00 \\
E-07\end{array}$ & unit & & RoW & $\begin{array}{l}\text { techno } \\
\text { sphere }\end{array}$ \\
\hline
\end{tabular}




\begin{tabular}{|c|c|c|c|c|c|c|}
\hline $\begin{array}{l}\text { heat, from municipal waste incineration to generic market for } \\
\text { heat district or industrial, other than natural gas }\end{array}$ & $\begin{array}{l}\text { heat, district or industrial, } \\
\text { other than natural gas }\end{array}$ & $\begin{array}{r}5.40 \\
E+03\end{array}$ & $\begin{array}{l}\text { megajoul } \\
\text { e }\end{array}$ & & ES & $\begin{array}{l}\text { techno } \\
\text { sphere }\end{array}$ \\
\hline market for air compressor, screw-type compressor, 300kW & $\begin{array}{l}\text { air compressor, screw-type } \\
\text { compressor, } 300 \mathrm{~kW}\end{array}$ & $\begin{array}{ll}4.35 \\
\mathrm{E}-06 \\
\end{array}$ & unit & & GLO & $\begin{array}{l}\text { techno } \\
\text { sphere }\end{array}$ \\
\hline market for electricity, low voltage & electricity, low voltage & $\begin{array}{r}2.42 \\
\mathrm{E}+01 \\
\end{array}$ & $\begin{array}{l}\text { kilowatt } \\
\text { hour }\end{array}$ & & ES & $\begin{array}{l}\text { techno } \\
\text { sphere }\end{array}$ \\
\hline market for gas turbine, $10 \mathrm{MW}$ electrical & $\begin{array}{l}\text { gas turbine, } 10 \mathrm{MW} \\
\text { electrical }\end{array}$ & $\begin{array}{l}2.54 \\
\mathrm{E}-08\end{array}$ & unit & & GLO & $\begin{array}{l}\text { techno } \\
\text { sphere }\end{array}$ \\
\hline sorbent, generic & & $\begin{array}{r}3.00 \\
\mathrm{E}+00 \\
\end{array}$ & kilogram & & RER & $\begin{array}{l}\text { techno } \\
\text { sphere }\end{array}$ \\
\hline transport, freight, lorry $>32$ metric ton, EURO 6 & $\begin{array}{l}\text { transport, freight, lorry } \\
>32 \text { metric ton, EURO6 }\end{array}$ & $\begin{array}{l}3.55 \\
\mathrm{E}-01 \\
\end{array}$ & $\begin{array}{l}\text { ton } \\
\text { kilomete } \\
r\end{array}$ & & RoW & $\begin{array}{l}\text { techno } \\
\text { sphere }\end{array}$ \\
\hline transport, freight, sea, container ship & $\begin{array}{l}\text { transport, freight, sea, } \\
\text { container ship }\end{array}$ & $\begin{array}{l}7.04 \\
\mathrm{E}-01 \\
\end{array}$ & $\begin{array}{l}\text { ton } \\
\text { kilomete } \\
\mathrm{r}\end{array}$ & & GLO & $\begin{array}{l}\text { techno } \\
\text { sphere }\end{array}$ \\
\hline transport, passengers, passenger aircraft, medium haul & $\begin{array}{l}\text { transport, passengers, } \\
\text { aircraft, medium haul }\end{array}$ & $\begin{array}{l}1.58 \\
\mathrm{E}-01 \\
\end{array}$ & $\begin{array}{l}\text { person } \\
\text { kilomete } \\
r\end{array}$ & & GLO & $\begin{array}{l}\text { techno } \\
\text { sphere }\end{array}$ \\
\hline $\begin{array}{l}\text { transport, pipeline, supercritical } \mathrm{CO} 2,200 \mathrm{~km} \mathrm{w} \\
\text { recompression }\end{array}$ & & $\begin{array}{r}3.00 \\
E+02 \\
\end{array}$ & $\begin{array}{l}\text { ton } \\
\text { kilomete } \\
\mathrm{r} \\
\end{array}$ & & RER & $\begin{array}{l}\text { techno } \\
\text { sphere }\end{array}$ \\
\hline $\begin{array}{l}\text { transport, pipeline, supercritical } \mathrm{CO} 2,200 \mathrm{~km} \text { w/o } \\
\text { recompression }\end{array}$ & & $\begin{array}{r}2.00 \\
\mathrm{E}+02 \\
\end{array}$ & $\begin{array}{l}\text { ton } \\
\text { kilomete } \\
\mathrm{r}\end{array}$ & & RER & $\begin{array}{l}\text { techno } \\
\text { sphere }\end{array}$ \\
\hline carbon dioxide, captured from atmosphere, HTHP + Grid, ES & & & ton & & ES & \\
\hline Name & Reference Product & $\begin{array}{l}\text { Amo } \\
\text { unt }\end{array}$ & Unit & Categories & $\begin{array}{l}\text { Loca } \\
\text { tion }\end{array}$ & Type \\
\hline Carbon dioxide, fossil & & $\begin{array}{r}7.00 \\
E+00 \\
\end{array}$ & kilogram & $\begin{array}{l}\text { air:urban air } \\
\text { close to ground }\end{array}$ & & $\begin{array}{l}\text { biosph } \\
\text { ere }\end{array}$ \\
\hline carbon dioxide capture system & & $\begin{array}{l}5.00 \\
\mathrm{E}-07 \\
\end{array}$ & unit & & RER & $\begin{array}{l}\text { techno } \\
\text { sphere }\end{array}$ \\
\hline drilling, deep borehole/m & & $\begin{array}{l}3.38 \\
\mathrm{E}-05 \\
\end{array}$ & meter & & RER & $\begin{array}{l}\text { techno } \\
\text { sphere }\end{array}$ \\
\hline end of life, carbon dioxide capture system & & $\begin{array}{l}5.00 \\
\mathrm{E}-07 \\
\end{array}$ & unit & & RoW & $\begin{array}{l}\text { techno } \\
\text { sphere }\end{array}$ \\
\hline heat pump production, brine-water, $\mathrm{CO} 2$ refrigerant, $10 \mathrm{~kW}$ & & $\begin{array}{l}8.56 \\
\mathrm{E}-04 \\
\end{array}$ & unit & & $\mathrm{CH}$ & $\begin{array}{l}\text { techno } \\
\text { sphere }\end{array}$ \\
\hline market for air compressor, screw-type compressor, $300 \mathrm{~kW}$ & $\begin{array}{l}\text { air compressor, screw-type } \\
\text { compressor, } 300 \mathrm{~kW}\end{array}$ & $\begin{array}{l}4.35 \\
\mathrm{E}-06 \\
\end{array}$ & unit & & GLO & $\begin{array}{l}\text { techno } \\
\text { sphere }\end{array}$ \\
\hline market for electricity, low voltage & electricity, low voltage & $\begin{array}{r}5.00 \\
E+02 \\
\end{array}$ & $\begin{array}{l}\text { kilowatt } \\
\text { hour }\end{array}$ & & ES & $\begin{array}{l}\text { techno } \\
\text { sphere }\end{array}$ \\
\hline market for electricity, low voltage & electricity, low voltage & $\begin{array}{r}1.14 \\
\mathrm{E}+02\end{array}$ & $\begin{array}{l}\text { kilowatt } \\
\text { hour }\end{array}$ & & ES & $\begin{array}{l}\text { techno } \\
\text { sphere }\end{array}$ \\
\hline market for electricity, low voltage & electricity, low voltage & $\begin{array}{r}5.17 \\
\mathrm{E}+02 \\
\end{array}$ & $\begin{array}{l}\text { kilowatt } \\
\text { hour }\end{array}$ & & ES & $\begin{array}{l}\text { techno } \\
\text { sphere }\end{array}$ \\
\hline market for electricity, low voltage & electricity, low voltage & $\begin{aligned} 2.42 \\
\mathrm{E}+01 \\
\end{aligned}$ & $\begin{array}{l}\text { kilowatt } \\
\text { hour }\end{array}$ & & ES & $\begin{array}{l}\text { techno } \\
\text { sphere }\end{array}$ \\
\hline market for gas turbine, $10 \mathrm{MW}$ electrical & $\begin{array}{l}\text { gas turbine, 10MW } \\
\text { electrical }\end{array}$ & $\begin{array}{ll}2.54 \\
\mathrm{E}-08 \\
\end{array}$ & unit & & GLO & $\begin{array}{l}\text { techno } \\
\text { sphere }\end{array}$ \\
\hline sorbent, generic & & $\begin{array}{r}3.00 \\
\mathrm{E}+00 \\
\end{array}$ & kilogram & & RER & $\begin{array}{l}\text { techno } \\
\text { sphere }\end{array}$ \\
\hline transport, freight, lorry $>32$ metric ton, EURO 6 & $\begin{array}{l}\text { transport, freight, lorry } \\
>32 \text { metric ton, EURO6 }\end{array}$ & $\begin{array}{l}3.55 \\
\mathrm{E}-01 \\
\end{array}$ & $\begin{array}{l}\text { ton } \\
\text { kilomete } \\
\mathrm{r}\end{array}$ & & RoW & $\begin{array}{l}\text { techno } \\
\text { sphere }\end{array}$ \\
\hline transport, freight, sea, container ship & $\begin{array}{l}\text { transport, freight, sea, } \\
\text { container ship }\end{array}$ & $\begin{array}{l}7.04 \\
\mathrm{E}-01 \\
\end{array}$ & $\begin{array}{l}\text { ton } \\
\text { kilomete } \\
\mathrm{r} \\
\end{array}$ & & GLO & $\begin{array}{l}\text { techno } \\
\text { sphere }\end{array}$ \\
\hline transport, passengers, passenger aircraft, medium haul & $\begin{array}{l}\text { transport, passengers, } \\
\text { aircraft, medium haul }\end{array}$ & $\begin{array}{l}1.58 \\
\mathrm{E}-01 \\
\end{array}$ & $\begin{array}{l}\text { person } \\
\text { kilomete } \\
\mathrm{r}\end{array}$ & & GLO & $\begin{array}{l}\text { techno } \\
\text { sphere }\end{array}$ \\
\hline $\begin{array}{l}\text { transport, pipeline, supercritical } \mathrm{CO} 2,200 \mathrm{~km} \mathrm{w} \\
\text { recompression }\end{array}$ & & $\begin{array}{r}3.00 \\
\mathrm{E}+02 \\
\end{array}$ & $\begin{array}{l}\text { ton } \\
\text { kilomete } \\
\mathrm{r}\end{array}$ & & RER & $\begin{array}{l}\text { techno } \\
\text { sphere }\end{array}$ \\
\hline $\begin{array}{l}\text { transport, pipeline, supercritical CO2, } 200 \mathrm{~km} \mathrm{w/o} \\
\text { recompression }\end{array}$ & & $\begin{array}{r}2.00 \\
E+02 \\
\end{array}$ & $\begin{array}{l}\text { ton } \\
\text { kilomete } \\
\mathrm{r}\end{array}$ & & RER & $\begin{array}{l}\text { techno } \\
\text { sphere }\end{array}$ \\
\hline $\begin{array}{l}\text { carbon dioxide, captured from atmosphere, Autonomous } \\
\text { Fresnel + PV, ES }\end{array}$ & & & ton & & ES & \\
\hline ( & Reference Product & $\begin{array}{l}\text { Amo } \\
\text { unt }\end{array}$ & Unit & Categories & $\begin{array}{l}\text { Loca } \\
\text { tion }\end{array}$ & Type \\
\hline Battery management system, kWh & & $\begin{array}{l}1.04 \\
E-01 \\
\end{array}$ & $\begin{array}{l}\text { kilowatt } \\
\text { hour }\end{array}$ & & GLO & $\begin{array}{l}\text { techno } \\
\text { sphere }\end{array}$ \\
\hline Carbon dioxide, fossil & & $\begin{array}{r}7.00 \\
E+00 \\
\end{array}$ & kilogram & $\begin{array}{l}\text { air:urban air } \\
\text { close to ground }\end{array}$ & & $\begin{array}{l}\text { biosph } \\
\text { ere }\end{array}$ \\
\hline
\end{tabular}




\begin{tabular}{|c|c|c|c|c|c|c|}
\hline Energy management system, kWh & & $\begin{array}{l}1.04 \\
\mathrm{E}-01 \\
\end{array}$ & $\begin{array}{l}\text { kilowatt } \\
\text { hour }\end{array}$ & & GLO & $\begin{array}{l}\text { techno } \\
\text { sphere }\end{array}$ \\
\hline Li-ion (NMC) & & $\begin{array}{l}1.04 \\
E-01\end{array}$ & $\begin{array}{l}\text { kilowatt } \\
\text { hour }\end{array}$ & & GLO & $\begin{array}{l}\text { techno } \\
\text { sphere }\end{array}$ \\
\hline Power conditioning system, container system & & $\begin{array}{l}3.13 \\
\mathrm{E}-02\end{array}$ & kW & & GLO & $\begin{array}{l}\text { techno } \\
\text { sphere }\end{array}$ \\
\hline carbon dioxide capture system & & $\begin{array}{l}5.00 \\
\mathrm{E}-07\end{array}$ & unit & & RER & $\begin{array}{l}\text { techno } \\
\text { sphere }\end{array}$ \\
\hline drilling, deep borehole/m & & $\begin{array}{l}3.38 \\
\mathrm{E}-05\end{array}$ & meter & & RER & $\begin{array}{l}\text { techno } \\
\text { sphere }\end{array}$ \\
\hline $\begin{array}{l}\text { electricity production, photovoltaic, } 570 \mathrm{kWp} \text { open ground } \\
\text { installation, multi-Si }\end{array}$ & electricity, low voltage & $\begin{array}{r}5.62 \\
\mathrm{E}+02 \\
\end{array}$ & $\begin{array}{l}\text { kilowatt } \\
\text { hour }\end{array}$ & & ES & $\begin{array}{l}\text { techno } \\
\text { sphere }\end{array}$ \\
\hline $\begin{array}{l}\text { electricity production, photovoltaic, } 570 \mathrm{kWp} \text { open ground } \\
\text { installation, multi-Si }\end{array}$ & electricity, low voltage & $\begin{array}{r}1.29 \\
E+02 \\
\end{array}$ & $\begin{array}{l}\text { kilowatt } \\
\text { hour }\end{array}$ & & ES & $\begin{array}{l}\text { techno } \\
\text { sphere }\end{array}$ \\
\hline end of life, carbon dioxide capture system & & $\begin{array}{l}5.00 \\
\mathrm{E}-07 \\
\end{array}$ & unit & & RoW & $\begin{array}{l}\text { techno } \\
\text { sphere }\end{array}$ \\
\hline $\begin{array}{l}\text { heat production, Fresnel solar collector plant, Tabernas, } \\
\text { Spain }\end{array}$ & $\begin{array}{l}\text { heat, central or small- } \\
\text { scale, Fresnel plant }\end{array}$ & $\begin{array}{r}5.40 \\
E+03 \\
\end{array}$ & $\begin{array}{l}\text { megajoul } \\
\mathrm{e}\end{array}$ & & ES & $\begin{array}{l}\text { techno } \\
\text { sphere }\end{array}$ \\
\hline market for air compressor, screw-type compressor, 300kW & $\begin{array}{l}\text { air compressor, screw-type } \\
\text { compressor, } 300 \mathrm{~kW}\end{array}$ & $\begin{array}{l}4.35 \\
\mathrm{E}-06 \\
\end{array}$ & unit & & GLO & $\begin{array}{l}\text { techno } \\
\text { sphere }\end{array}$ \\
\hline market for electricity, low voltage & electricity, low voltage & $\begin{array}{r}2.42 \\
\mathrm{E}+01 \\
\end{array}$ & $\begin{array}{l}\text { kilowatt } \\
\text { hour }\end{array}$ & & ES & $\begin{array}{l}\text { techno } \\
\text { sphere }\end{array}$ \\
\hline market for gas turbine, $10 \mathrm{MW}$ electrical & $\begin{array}{l}\text { gas turbine, } 10 \mathrm{MW} \\
\text { electrical }\end{array}$ & $\begin{array}{l}2.54 \\
\mathrm{E}-08 \\
\end{array}$ & unit & & GLO & $\begin{array}{l}\text { techno } \\
\text { sphere }\end{array}$ \\
\hline sorbent, generic & & $\begin{array}{r}3.00 \\
E+00\end{array}$ & kilogram & & RER & $\begin{array}{l}\text { techno } \\
\text { sphere }\end{array}$ \\
\hline storage unit production, Fresnel plant, Jordan, $12 \mathrm{~h}$ storage & $\begin{array}{l}\text { storage unit, Fresnel plant, } \\
\text { Jordan, } 12 \mathrm{~h} \text { storage }\end{array}$ & $\begin{array}{l}4.00 \\
E-07\end{array}$ & unit & & JO & $\begin{array}{l}\text { techno } \\
\text { sphere }\end{array}$ \\
\hline transport, freight, lorry >32 metric ton, EURO6 & $\begin{array}{l}\text { transport, freight, lorry } \\
>32 \text { metric ton, EURO6 }\end{array}$ & $\begin{array}{l}3.55 \\
\mathrm{E}-01\end{array}$ & $\begin{array}{l}\text { ton } \\
\text { kilomete } \\
\mathrm{r}\end{array}$ & & RoW & $\begin{array}{l}\text { techno } \\
\text { sphere }\end{array}$ \\
\hline transport, freight, sea, container ship & $\begin{array}{l}\text { transport, freight, sea, } \\
\text { container ship }\end{array}$ & $\begin{array}{l}7.04 \\
\mathrm{E}-01 \\
\end{array}$ & $\begin{array}{l}\text { ton } \\
\text { kilomete } \\
r\end{array}$ & & GLO & $\begin{array}{l}\text { techno } \\
\text { sphere }\end{array}$ \\
\hline transport, passengers, passenger aircraft, medium haul & $\begin{array}{l}\text { transport, passengers, } \\
\text { aircraft, medium haul }\end{array}$ & $\begin{array}{l}1.58 \\
\mathrm{E}-01 \\
\end{array}$ & $\begin{array}{l}\text { person } \\
\text { kilomete } \\
r\end{array}$ & & GLO & $\begin{array}{l}\text { techno } \\
\text { sphere }\end{array}$ \\
\hline $\begin{array}{l}\text { transport, pipeline, supercritical CO2, } 200 \mathrm{~km} \mathrm{w} \\
\text { recompression }\end{array}$ & & $\begin{array}{r}3.00 \\
E+02\end{array}$ & $\begin{array}{l}\text { ton } \\
\text { kilomete } \\
r\end{array}$ & & RER & $\begin{array}{l}\text { techno } \\
\text { sphere }\end{array}$ \\
\hline $\begin{array}{l}\text { transport, pipeline, supercritical CO2, } 200 \mathrm{~km} \text { w/o } \\
\text { recompression }\end{array}$ & & $\begin{array}{r}2.00 \\
E+02\end{array}$ & $\begin{array}{l}\text { ton } \\
\text { kilomete } \\
\mathrm{r}\end{array}$ & & RER & $\begin{array}{l}\text { techno } \\
\text { sphere }\end{array}$ \\
\hline $\begin{array}{l}\text { carbon dioxide, captured from atmosphere, Autonomous } \\
\text { HTHP + PV, ES }\end{array}$ & & & ton & & ES & \\
\hline Name & Reference Product & $\begin{array}{l}\text { Amo } \\
\text { unt }\end{array}$ & Unit & Categories & $\begin{array}{l}\text { Loca } \\
\text { tion }\end{array}$ & Type \\
\hline Battery management system, kWh & & $\begin{array}{l}1.84 \\
E-01\end{array}$ & $\begin{array}{l}\text { kilowatt } \\
\text { hour }\end{array}$ & & GLO & $\begin{array}{l}\text { techno } \\
\text { sphere }\end{array}$ \\
\hline Carbon dioxide, fossil & & $\begin{array}{r}7.00 \\
E+00 \\
\end{array}$ & kilogram & $\begin{array}{l}\text { air:urban air } \\
\text { close to ground }\end{array}$ & & $\begin{array}{l}\text { biosph } \\
\text { ere }\end{array}$ \\
\hline Energy management system, kWh & & $\begin{array}{ll}1.84 \\
\mathrm{E}-01 \\
\end{array}$ & $\begin{array}{l}\text { kilowatt } \\
\text { hour }\end{array}$ & & GLO & $\begin{array}{l}\text { techno } \\
\text { sphere }\end{array}$ \\
\hline Li-ion (NMC) & & $\begin{array}{ll}1.84 \\
\mathrm{E}-01 \\
\end{array}$ & $\begin{array}{l}\text { kilowatt } \\
\text { hour }\end{array}$ & & GLO & $\begin{array}{l}\text { techno } \\
\text { sphere }\end{array}$ \\
\hline Power conditioning system, container system & & $\begin{array}{l}5.52 \\
\mathrm{E}-02 \\
\end{array}$ & $\mathrm{~kW}$ & & GLO & $\begin{array}{l}\text { techno } \\
\text { sphere }\end{array}$ \\
\hline carbon dioxide capture system & & $\begin{array}{l}5.00 \\
\mathrm{E}-07 \\
\end{array}$ & unit & & RER & $\begin{array}{l}\text { techno } \\
\text { sphere }\end{array}$ \\
\hline drilling, deep borehole/m & & $\begin{array}{l}3.38 \\
\mathrm{E}-05 \\
\end{array}$ & meter & & RER & $\begin{array}{l}\text { techno } \\
\text { sphere }\end{array}$ \\
\hline $\begin{array}{l}\text { electricity production, photovoltaic, } 570 \mathrm{kWp} \text { open ground } \\
\text { installation, multi-Si }\end{array}$ & electricity, low voltage & $\begin{array}{r}5.62 \\
\mathrm{E}+02 \\
\end{array}$ & $\begin{array}{l}\text { kilowatt } \\
\text { hour }\end{array}$ & & ES & $\begin{array}{l}\text { techno } \\
\text { sphere }\end{array}$ \\
\hline $\begin{array}{l}\text { electricity production, photovoltaic, } 570 \mathrm{kWp} \text { open ground } \\
\text { installation, multi-Si }\end{array}$ & electricity, low voltage & $\begin{array}{r}1.29 \\
\mathrm{E}+02 \\
\end{array}$ & $\begin{array}{l}\text { kilowatt } \\
\text { hour }\end{array}$ & & ES & $\begin{array}{l}\text { techno } \\
\text { sphere }\end{array}$ \\
\hline $\begin{array}{l}\text { electricity production, photovoltaic, } 570 \mathrm{kWp} \text { open ground } \\
\text { installation, multi-Si }\end{array}$ & electricity, low voltage & $\begin{array}{r}5.81 \\
E+02 \\
\end{array}$ & $\begin{array}{l}\text { kilowatt } \\
\text { hour }\end{array}$ & & ES & $\begin{array}{l}\text { techno } \\
\text { sphere }\end{array}$ \\
\hline end of life, carbon dioxide capture system & & $\begin{array}{l}5.00 \\
\mathrm{E}-07 \\
\end{array}$ & unit & & RoW & $\begin{array}{l}\text { techno } \\
\text { sphere }\end{array}$ \\
\hline heat pump production, brine-water, $\mathrm{CO} 2$ refrigerant, $10 \mathrm{~kW}$ & & $\begin{array}{l}8.56 \\
\mathrm{E}-04\end{array}$ & unit & & $\mathrm{CH}$ & $\begin{array}{l}\text { techno } \\
\text { sphere }\end{array}$ \\
\hline market for air compressor, screw-type compressor, 300kW & $\begin{array}{l}\text { air compressor, screw-type } \\
\text { compressor, } 300 \mathrm{~kW}\end{array}$ & $\begin{array}{l}4.35 \\
\mathrm{E}-06\end{array}$ & unit & & GLO & $\begin{array}{l}\text { techno } \\
\text { sphere }\end{array}$ \\
\hline market for electricity, low voltage & electricity, low voltage & $\begin{array}{r}2.42 \\
E+01\end{array}$ & $\begin{array}{l}\text { kilowatt } \\
\text { hour }\end{array}$ & & ES & $\begin{array}{l}\text { techno } \\
\text { sphere }\end{array}$ \\
\hline market for gas turbine, $10 \mathrm{MW}$ electrical & $\begin{array}{l}\text { gas turbine, } 10 \mathrm{MW} \\
\text { electrical }\end{array}$ & $\begin{array}{l}2.54 \\
\mathrm{E}-08 \\
\end{array}$ & unit & & GLO & $\begin{array}{l}\text { techno } \\
\text { sphere }\end{array}$ \\
\hline
\end{tabular}




\begin{tabular}{|c|c|c|c|c|c|c|}
\hline sorbent, generic & & $\begin{array}{r}3.00 \\
E+00 \\
\end{array}$ & kilogram & & RER & $\begin{array}{l}\text { techno } \\
\text { sphere }\end{array}$ \\
\hline transport, freight, lorry $>32$ metric ton, EURO 6 & $\begin{array}{l}\text { transport, freight, lorry } \\
>32 \text { metric ton, EURO6 }\end{array}$ & $\begin{array}{l}3.55 \\
\mathrm{E}-01\end{array}$ & $\begin{array}{l}\text { ton } \\
\text { kilomete } \\
\mathrm{r}\end{array}$ & & RoW & $\begin{array}{l}\text { techno } \\
\text { sphere }\end{array}$ \\
\hline transport, freight, sea, container ship & $\begin{array}{l}\text { transport, freight, sea, } \\
\text { container ship }\end{array}$ & $\begin{array}{l}7.04 \\
\mathrm{E}-01 \\
\end{array}$ & $\begin{array}{l}\text { ton } \\
\text { kilomete } \\
\mathrm{r}\end{array}$ & & GLO & $\begin{array}{l}\text { techno } \\
\text { sphere }\end{array}$ \\
\hline transport, passengers, passenger aircraft, medium haul & $\begin{array}{l}\text { transport, passengers, } \\
\text { aircraft, medium haul }\end{array}$ & $\begin{array}{l}1.58 \\
E-01\end{array}$ & $\begin{array}{l}\text { person } \\
\text { kilomete } \\
r\end{array}$ & & GLO & $\begin{array}{l}\text { techno } \\
\text { sphere }\end{array}$ \\
\hline $\begin{array}{l}\text { transport, pipeline, supercritical } \mathrm{CO} 2,200 \mathrm{~km} \mathrm{w} \\
\text { recompression }\end{array}$ & & $\begin{array}{r}3.00 \\
\mathrm{E}+02 \\
\end{array}$ & $\begin{array}{l}\text { ton } \\
\text { kilomete } \\
\mathrm{r}\end{array}$ & & RER & $\begin{array}{l}\text { techno } \\
\text { sphere }\end{array}$ \\
\hline $\begin{array}{l}\text { transport, pipeline, supercritical CO2, 200km w/o } \\
\text { recompression }\end{array}$ & & $\begin{array}{r}2.00 \\
E+02\end{array}$ & $\begin{array}{l}\text { ton } \\
\text { kilomete } \\
\mathrm{r}\end{array}$ & & RER & $\begin{array}{l}\text { techno } \\
\text { sphere }\end{array}$ \\
\hline $\begin{array}{l}\text { carbon dioxide, captured from atmosphere, Waste heat + } \\
\text { Grid, } \mathrm{CH}\end{array}$ & & & ton & & $\mathrm{CH}$ & \\
\hline Name & Reference Product & $\begin{array}{l}\text { Amo } \\
\text { unt }\end{array}$ & Unit & Categories & $\begin{array}{l}\text { Loca } \\
\text { tion }\end{array}$ & Type \\
\hline Carbon dioxide, fossil & & $\begin{array}{r}2.10 \\
\mathrm{E}+01 \\
\end{array}$ & kilogram & $\begin{array}{l}\text { air:urban air } \\
\text { close to ground }\end{array}$ & & $\begin{array}{l}\text { biosph } \\
\text { ere }\end{array}$ \\
\hline carbon dioxide capture system & & $\begin{array}{l}5.00 \\
\mathrm{E}-07\end{array}$ & unit & & RER & $\begin{array}{l}\text { techno } \\
\text { sphere }\end{array}$ \\
\hline drilling, deep borehole/m & & $\begin{array}{l}3.38 \\
E-05\end{array}$ & meter & & RER & $\begin{array}{l}\text { techno } \\
\text { sphere }\end{array}$ \\
\hline end of life, carbon dioxide capture system & & $\begin{array}{l}5.00 \\
E-07\end{array}$ & unit & & RoW & $\begin{array}{l}\text { techno } \\
\text { sphere }\end{array}$ \\
\hline $\begin{array}{l}\text { heat, from municipal waste incineration to generic market for } \\
\text { heat district or industrial, other than natural gas }\end{array}$ & $\begin{array}{l}\text { heat, district or industrial, } \\
\text { other than natural gas }\end{array}$ & $\begin{array}{r}5.40 \\
\mathrm{E}+03 \\
\end{array}$ & $\begin{array}{l}\text { megajoul } \\
\mathrm{e}\end{array}$ & & $\mathrm{CH}$ & $\begin{array}{l}\text { techno } \\
\text { sphere }\end{array}$ \\
\hline market for air compressor, screw-type compressor, $300 \mathrm{~kW}$ & $\begin{array}{l}\text { air compressor, screw-type } \\
\text { compressor, } 300 \mathrm{~kW}\end{array}$ & $\begin{array}{l}4.35 \\
\mathrm{E}-06 \\
\end{array}$ & unit & & GLO & $\begin{array}{l}\text { techno } \\
\text { sphere }\end{array}$ \\
\hline market for electricity, low voltage & electricity, low voltage & $\begin{array}{r}5.00 \\
\mathrm{E}+02 \\
\end{array}$ & $\begin{array}{l}\text { kilowatt } \\
\text { hour }\end{array}$ & & $\mathrm{CH}$ & $\begin{array}{l}\text { techno } \\
\text { sphere }\end{array}$ \\
\hline market for electricity, low voltage & electricity, low voltage & $\begin{array}{r}1.14 \\
\mathrm{E}+02 \\
\end{array}$ & $\begin{array}{l}\text { kilowatt } \\
\text { hour }\end{array}$ & & $\mathrm{CH}$ & $\begin{array}{l}\text { techno } \\
\text { sphere }\end{array}$ \\
\hline market for electricity, low voltage & electricity, low voltage & $\begin{array}{r}2.42 \\
E+01\end{array}$ & $\begin{array}{l}\text { kilowatt } \\
\text { hour }\end{array}$ & & NO & $\begin{array}{l}\text { techno } \\
\text { sphere }\end{array}$ \\
\hline market for gas turbine, $10 \mathrm{MW}$ electrical & $\begin{array}{l}\text { gas turbine, 10MW } \\
\text { electrical }\end{array}$ & $\begin{array}{l}2.54 \\
\mathrm{E}-08 \\
\end{array}$ & unit & & GLO & $\begin{array}{l}\text { techno } \\
\text { sphere }\end{array}$ \\
\hline sorbent, generic & & $\begin{array}{r}3.00 \\
E+00 \\
\end{array}$ & kilogram & & RER & $\begin{array}{l}\text { techno } \\
\text { sphere }\end{array}$ \\
\hline transport, freight, lorry $>32$ metric ton, EURO 6 & $\begin{array}{l}\text { transport, freight, lorry } \\
>32 \text { metric ton, EURO6 }\end{array}$ & $\begin{array}{l}7.44 \\
\mathrm{E}-02 \\
\end{array}$ & $\begin{array}{l}\text { ton } \\
\text { kilomete } \\
\mathrm{r}\end{array}$ & & RoW & $\begin{array}{l}\text { techno } \\
\text { sphere }\end{array}$ \\
\hline $\begin{array}{l}\text { transport, pipeline, supercritical } \mathrm{CO} 2,200 \mathrm{~km} \mathrm{w} \\
\text { recompression }\end{array}$ & & $\begin{array}{r}1.30 \\
\mathrm{E}+03 \\
\end{array}$ & $\begin{array}{l}\text { ton } \\
\text { kilomete } \\
\mathrm{r}\end{array}$ & & RER & $\begin{array}{l}\text { techno } \\
\text { sphere }\end{array}$ \\
\hline $\begin{array}{l}\text { transport, pipeline, supercritical CO2, } 200 \mathrm{~km} \text { w/o } \\
\text { recompression }\end{array}$ & & $\begin{array}{r}2.00 \\
E+02 \\
\end{array}$ & $\begin{array}{l}\text { ton } \\
\text { kilomete } \\
\mathrm{r}\end{array}$ & & RER & $\begin{array}{l}\text { techno } \\
\text { sphere }\end{array}$ \\
\hline carbon dioxide, captured from atmosphere, HTHP + Grid, CH & & & ton & & $\mathrm{CH}$ & \\
\hline 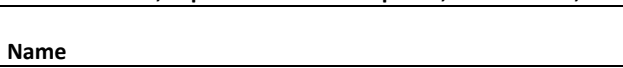 & Reference Product & $\begin{array}{l}\text { Amo } \\
\text { unt }\end{array}$ & Unit & Categories & $\begin{array}{l}\text { Loca } \\
\text { tion }\end{array}$ & Type \\
\hline Carbon dioxide, fossil & & $\begin{aligned} 2.10 \\
\mathrm{E}+01 \\
\end{aligned}$ & kilogram & $\begin{array}{l}\text { air:urban air } \\
\text { close to ground }\end{array}$ & & $\begin{array}{l}\text { biosph } \\
\text { ere }\end{array}$ \\
\hline carbon dioxide capture system & & $\begin{array}{ll}5.00 \\
\mathrm{E}-07 \\
\end{array}$ & unit & & RER & $\begin{array}{l}\text { techno } \\
\text { sphere }\end{array}$ \\
\hline drilling, deep borehole/m & & $\begin{array}{l}3.38 \\
\mathrm{E}-05\end{array}$ & meter & & RER & $\begin{array}{l}\text { techno } \\
\text { sphere }\end{array}$ \\
\hline end of life, carbon dioxide capture system & & $\begin{array}{l}5.00 \\
\mathrm{E}-07\end{array}$ & unit & & RoW & $\begin{array}{l}\text { techno } \\
\text { sphere }\end{array}$ \\
\hline heat pump production, brine-water, $\mathrm{CO} 2$ refrigerant, $10 \mathrm{~kW}$ & & $\begin{array}{l}8.56 \\
\mathrm{E}-04 \\
\end{array}$ & unit & & $\mathrm{CH}$ & $\begin{array}{l}\text { techno } \\
\text { sphere }\end{array}$ \\
\hline market for air compressor, screw-type compressor, 300kW & $\begin{array}{l}\text { air compressor, screw-type } \\
\text { compressor, } 300 \mathrm{~kW}\end{array}$ & $\begin{array}{l}4.35 \\
\mathrm{E}-06 \\
\end{array}$ & unit & & GLO & $\begin{array}{l}\text { techno } \\
\text { sphere }\end{array}$ \\
\hline market for electricity, low voltage & electricity, low voltage & $\begin{array}{r}5.00 \\
\mathrm{E}+02 \\
\end{array}$ & $\begin{array}{l}\text { kilowatt } \\
\text { hour }\end{array}$ & & $\mathrm{CH}$ & $\begin{array}{l}\text { techno } \\
\text { sphere }\end{array}$ \\
\hline market for electricity, low voltage & electricity, low voltage & $\begin{array}{r}1.14 \\
E+02\end{array}$ & $\begin{array}{l}\text { kilowatt } \\
\text { hour }\end{array}$ & & $\mathrm{CH}$ & $\begin{array}{l}\text { techno } \\
\text { sphere }\end{array}$ \\
\hline market for electricity, low voltage & electricity, low voltage & $\begin{array}{r}5.17 \\
E+02\end{array}$ & $\begin{array}{l}\text { kilowatt } \\
\text { hour }\end{array}$ & & $\mathrm{CH}$ & $\begin{array}{l}\text { techno } \\
\text { sphere }\end{array}$ \\
\hline market for electricity, low voltage & electricity, low voltage & $\begin{array}{r}2.42 \\
E+01\end{array}$ & $\begin{array}{l}\text { kilowatt } \\
\text { hour }\end{array}$ & & NO & $\begin{array}{l}\text { techno } \\
\text { sphere }\end{array}$ \\
\hline
\end{tabular}




\begin{tabular}{|c|c|c|c|c|c|c|}
\hline market for gas turbine, $10 \mathrm{MW}$ electrical & $\begin{array}{l}\text { gas turbine, } 10 \mathrm{MW} \\
\text { electrical }\end{array}$ & $\begin{array}{l}2.54 \\
\mathrm{E}-08 \\
\end{array}$ & unit & & GLO & $\begin{array}{l}\text { techno } \\
\text { sphere }\end{array}$ \\
\hline sorbent, generic & & $\begin{array}{r}3.00 \\
E+00 \\
\end{array}$ & kilogram & & RER & $\begin{array}{l}\text { techno } \\
\text { sphere }\end{array}$ \\
\hline transport, freight, lorry >32 metric ton, EURO6 & $\begin{array}{l}\text { transport, freight, lorry } \\
>32 \text { metric ton, EURO6 }\end{array}$ & $\begin{array}{l}7.44 \\
\mathrm{E}-02 \\
\end{array}$ & $\begin{array}{l}\text { ton } \\
\text { kilomete } \\
\mathrm{r}\end{array}$ & & RoW & $\begin{array}{l}\text { techno } \\
\text { sphere }\end{array}$ \\
\hline $\begin{array}{l}\text { transport, pipeline, supercritical } \mathrm{CO} 2,200 \mathrm{~km} \mathrm{w} \\
\text { recompression }\end{array}$ & & $\begin{array}{r}1.30 \\
\mathrm{E}+03 \\
\end{array}$ & $\begin{array}{l}\text { ton } \\
\text { kilomete } \\
\mathrm{r}\end{array}$ & & RER & $\begin{array}{l}\text { techno } \\
\text { sphere }\end{array}$ \\
\hline $\begin{array}{l}\text { transport, pipeline, supercritical CO2, } 200 \mathrm{~km} \text { w/o } \\
\text { recompression }\end{array}$ & & $\begin{array}{r}2.00 \\
E+02 \\
\end{array}$ & $\begin{array}{l}\text { ton } \\
\text { kilomete } \\
r\end{array}$ & & RER & $\begin{array}{l}\text { techno } \\
\text { sphere }\end{array}$ \\
\hline $\begin{array}{l}\text { carbon dioxide, captured from atmosphere, Waste heat + } \\
\text { Grid, } \mathrm{CL}\end{array}$ & & & ton & & $\mathrm{CL}$ & \\
\hline 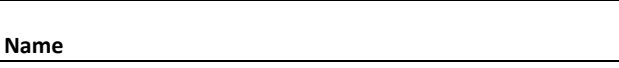 & Reference Product & $\begin{array}{l}\text { Amo } \\
\text { unt }\end{array}$ & Unit & Categories & $\begin{array}{l}\text { Loca } \\
\text { tion }\end{array}$ & Type \\
\hline Carbon dioxide, fossil & & $\begin{array}{r}2.10 \\
E+01 \\
\end{array}$ & kilogram & $\begin{array}{l}\text { air:urban air } \\
\text { close to ground }\end{array}$ & & $\begin{array}{l}\text { biosph } \\
\text { ere }\end{array}$ \\
\hline carbon dioxide capture system & & $\begin{array}{l}5.00 \\
\mathrm{E}-07 \\
\end{array}$ & unit & & RER & $\begin{array}{l}\text { techno } \\
\text { sphere }\end{array}$ \\
\hline drilling, deep borehole/m & & $\begin{array}{ll}3.38 \\
\mathrm{E}-05 \\
\end{array}$ & meter & & RER & $\begin{array}{l}\text { techno } \\
\text { sphere }\end{array}$ \\
\hline end of life, carbon dioxide capture system & & $\begin{array}{l}5.00 \\
\mathrm{E}-07 \\
\end{array}$ & unit & & RoW & $\begin{array}{l}\text { techno } \\
\text { sphere }\end{array}$ \\
\hline $\begin{array}{l}\text { heat, from municipal waste incineration to generic market for } \\
\text { heat district or industrial, other than natural gas }\end{array}$ & $\begin{array}{l}\text { heat, district or industrial, } \\
\text { other than natural gas }\end{array}$ & $\begin{array}{r}5.40 \\
E+03 \\
\end{array}$ & $\begin{array}{l}\text { megajoul } \\
\mathrm{e}\end{array}$ & & RoW & $\begin{array}{l}\text { techno } \\
\text { sphere }\end{array}$ \\
\hline market for air compressor, screw-type compressor, 300kW & $\begin{array}{l}\text { air compressor, screw-type } \\
\text { compressor, } 300 \mathrm{~kW}\end{array}$ & $\begin{array}{l}4.35 \\
\mathrm{E}-06 \\
\end{array}$ & unit & & GLO & $\begin{array}{l}\text { techno } \\
\text { sphere }\end{array}$ \\
\hline market for electricity, low voltage & electricity, low voltage & $\begin{array}{r}5.00 \\
E+02 \\
\end{array}$ & $\begin{array}{l}\text { kilowatt } \\
\text { hour }\end{array}$ & & $\mathrm{CL}$ & $\begin{array}{l}\text { techno } \\
\text { sphere }\end{array}$ \\
\hline market for electricity, low voltage & electricity, low voltage & $\begin{array}{r}1.14 \\
E+02 \\
\end{array}$ & $\begin{array}{l}\text { kilowatt } \\
\text { hour }\end{array}$ & & $\mathrm{CL}$ & $\begin{array}{l}\text { techno } \\
\text { sphere }\end{array}$ \\
\hline market for gas turbine, $10 \mathrm{MW}$ electrical & $\begin{array}{l}\text { gas turbine, } 10 \mathrm{MW} \\
\text { electrical }\end{array}$ & $\begin{array}{l}2.54 \\
\mathrm{E}-08 \\
\end{array}$ & unit & & GLO & $\begin{array}{l}\text { techno } \\
\text { sphere }\end{array}$ \\
\hline market group for electricity, low voltage & electricity, low voltage & $\begin{array}{r}2.42 \\
E+01\end{array}$ & $\begin{array}{l}\text { kilowatt } \\
\text { hour }\end{array}$ & & $\mathrm{BR}$ & $\begin{array}{l}\text { techno } \\
\text { sphere }\end{array}$ \\
\hline sorbent, generic & & $\begin{array}{r}3.00 \\
E+00\end{array}$ & kilogram & & RER & $\begin{array}{l}\text { techno } \\
\text { sphere }\end{array}$ \\
\hline transport, freight, lorry >32 metric ton, EURO6 & $\begin{array}{l}\text { transport, freight, lorry } \\
>32 \text { metric ton, EURO6 }\end{array}$ & $\begin{array}{l}2.53 \\
\mathrm{E}-01\end{array}$ & $\begin{array}{l}\text { ton } \\
\text { kilomete } \\
r\end{array}$ & & RoW & $\begin{array}{l}\text { techno } \\
\text { sphere }\end{array}$ \\
\hline transport, freight, sea, container ship & $\begin{array}{l}\text { transport, freight, sea, } \\
\text { container ship }\end{array}$ & $\begin{array}{r}8.21 \\
E+00 \\
\end{array}$ & $\begin{array}{l}\text { ton } \\
\text { kilomete } \\
\mathrm{r}\end{array}$ & & GLO & $\begin{array}{l}\text { techno } \\
\text { sphere }\end{array}$ \\
\hline transport, passengers, passenger aircraft, medium haul & $\begin{array}{l}\text { transport, passengers, } \\
\text { aircraft, medium haul }\end{array}$ & $\begin{array}{r}1.02 \\
E+00 \\
\end{array}$ & $\begin{array}{l}\text { person } \\
\text { kilomete } \\
r\end{array}$ & & GLO & $\begin{array}{l}\text { techno } \\
\text { sphere }\end{array}$ \\
\hline $\begin{array}{l}\text { transport, pipeline, supercritical CO2, } 200 \mathrm{~km} \mathrm{w} \\
\text { recompression }\end{array}$ & & $\begin{array}{r}1.30 \\
E+03\end{array}$ & $\begin{array}{l}\text { ton } \\
\text { kilomete } \\
\mathrm{r}\end{array}$ & & RER & $\begin{array}{l}\text { techno } \\
\text { sphere }\end{array}$ \\
\hline $\begin{array}{l}\text { transport, pipeline, supercritical } \mathrm{CO} 2,200 \mathrm{~km} \text { w/o } \\
\text { recompression }\end{array}$ & & $\begin{array}{r}2.00 \\
E+02\end{array}$ & $\begin{array}{l}\text { ton } \\
\text { kilomete } \\
\mathrm{r}\end{array}$ & & RER & $\begin{array}{l}\text { techno } \\
\text { sphere }\end{array}$ \\
\hline $\begin{array}{l}\text { carbon dioxide, captured from atmosphere, Waste heat + } \\
\text { PV + Battery, CL }\end{array}$ & & & ton & & $\mathrm{CL}$ & \\
\hline Name & Reference Product & $\begin{array}{l}\text { Amo } \\
\text { unt }\end{array}$ & Unit & Categories & $\begin{array}{l}\text { Loca } \\
\text { tion }\end{array}$ & Type \\
\hline Battery management system, kWh & & $\begin{array}{l}9.99 \\
\mathrm{E}-02 \\
\end{array}$ & $\begin{array}{l}\text { kilowatt } \\
\text { hour }\end{array}$ & & GLO & $\begin{array}{l}\text { techno } \\
\text { sphere }\end{array}$ \\
\hline Carbon dioxide, fossil & & $\begin{array}{r}2.10 \\
E+01\end{array}$ & kilogram & $\begin{array}{l}\text { air:urban air } \\
\text { close to ground }\end{array}$ & & $\begin{array}{l}\text { biosph } \\
\text { ere }\end{array}$ \\
\hline Energy management system, kWh & & $\begin{array}{l}9.99 \\
\mathrm{E}-02 \\
\end{array}$ & $\begin{array}{l}\text { kilowatt } \\
\text { hour }\end{array}$ & & GLO & $\begin{array}{l}\text { techno } \\
\text { sphere }\end{array}$ \\
\hline Li-ion (NMC) & & $\begin{array}{l}9.99 \\
\mathrm{E}-02 \\
\end{array}$ & $\begin{array}{l}\text { kilowatt } \\
\text { hour }\end{array}$ & & GLO & $\begin{array}{l}\text { techno } \\
\text { sphere }\end{array}$ \\
\hline Power conditioning system, container system & & $\begin{array}{l}3.00 \\
\mathrm{E}-02\end{array}$ & $\mathrm{~kW}$ & & GLO & $\begin{array}{l}\text { techno } \\
\text { sphere }\end{array}$ \\
\hline carbon dioxide capture system & & $\begin{array}{ll}5.00 \\
\mathrm{E}-07\end{array}$ & unit & & RER & $\begin{array}{l}\text { techno } \\
\text { sphere }\end{array}$ \\
\hline drilling, deep borehole/m & & $\begin{array}{ll}3.38 \\
\mathrm{E}-05 \\
\end{array}$ & meter & & RER & $\begin{array}{l}\text { techno } \\
\text { sphere }\end{array}$ \\
\hline $\begin{array}{l}\text { electricity production, photovoltaic, } 570 \mathrm{kWp} \text { open ground } \\
\text { installation, multi-Si }\end{array}$ & electricity, low voltage & $\begin{array}{r}5.62 \\
E+02 \\
\end{array}$ & $\begin{array}{l}\text { kilowatt } \\
\text { hour }\end{array}$ & & $\mathrm{CL}$ & $\begin{array}{l}\text { techno } \\
\text { sphere }\end{array}$ \\
\hline $\begin{array}{l}\text { electricity production, photovoltaic, } 570 \mathrm{kWp} \text { open ground } \\
\text { installation, multi-Si }\end{array}$ & electricity, low voltage & $\begin{array}{r}1.29 \\
E+02 \\
\end{array}$ & $\begin{array}{l}\text { kilowatt } \\
\text { hour }\end{array}$ & & $\mathrm{CL}$ & $\begin{array}{l}\text { techno } \\
\text { sphere }\end{array}$ \\
\hline
\end{tabular}




\begin{tabular}{|c|c|c|c|c|c|c|}
\hline end of life, carbon dioxide capture system & & $\begin{array}{l}5.00 \\
\mathrm{E}-07\end{array}$ & unit & & RoW & $\begin{array}{l}\text { techno } \\
\text { sphere }\end{array}$ \\
\hline $\begin{array}{l}\text { heat, from municipal waste incineration to generic market for } \\
\text { heat district or industrial, other than natural gas }\end{array}$ & $\begin{array}{l}\text { heat, district or industrial, } \\
\text { other than natural gas }\end{array}$ & $\begin{aligned} 5.40 \\
E+03\end{aligned}$ & $\begin{array}{l}\text { megajoul } \\
\mathrm{e}\end{array}$ & & RoW & $\begin{array}{l}\text { techno } \\
\text { sphere }\end{array}$ \\
\hline market for air compressor, screw-type compressor, 300kW & $\begin{array}{l}\text { air compressor, screw-type } \\
\text { compressor, } 300 \mathrm{~kW}\end{array}$ & $\begin{array}{l}4.35 \\
\mathrm{E}-06 \\
\end{array}$ & unit & & GLO & $\begin{array}{l}\text { techno } \\
\text { sphere }\end{array}$ \\
\hline market for gas turbine, $10 \mathrm{MW}$ electrical & $\begin{array}{l}\text { gas turbine, 10MW } \\
\text { electrical }\end{array}$ & $\begin{array}{l}2.54 \\
\mathrm{E}-08 \\
\end{array}$ & unit & & GLO & $\begin{array}{l}\text { techno } \\
\text { sphere }\end{array}$ \\
\hline market group for electricity, low voltage & electricity, low voltage & $\begin{array}{r}2.42 \\
E+01\end{array}$ & $\begin{array}{l}\text { kilowatt } \\
\text { hour }\end{array}$ & & $\mathrm{BR}$ & $\begin{array}{l}\text { techno } \\
\text { sphere }\end{array}$ \\
\hline sorbent, generic & & $\begin{array}{r}3.00 \\
\mathrm{E}+00 \\
\end{array}$ & kilogram & & RER & $\begin{array}{l}\text { techno } \\
\text { sphere }\end{array}$ \\
\hline transport, freight, lorry $>32$ metric ton, EURO 6 & $\begin{array}{l}\text { transport, freight, lorry } \\
>32 \text { metric ton, EURO6 }\end{array}$ & $\begin{array}{l}2.53 \\
E-01\end{array}$ & $\begin{array}{l}\text { ton } \\
\text { kilomete } \\
r\end{array}$ & & RoW & $\begin{array}{l}\text { techno } \\
\text { sphere }\end{array}$ \\
\hline transport, freight, sea, container ship & $\begin{array}{l}\text { transport, freight, sea, } \\
\text { container ship }\end{array}$ & $\begin{array}{r}8.21 \\
E+00\end{array}$ & $\begin{array}{l}\text { ton } \\
\text { kilomete } \\
r\end{array}$ & & GLO & $\begin{array}{l}\text { techno } \\
\text { sphere }\end{array}$ \\
\hline transport, passengers, passenger aircraft, medium haul & $\begin{array}{l}\text { transport, passengers, } \\
\text { aircraft, medium haul }\end{array}$ & $\begin{array}{r}1.02 \\
\mathrm{E}+00 \\
\end{array}$ & $\begin{array}{l}\text { person } \\
\text { kilomete } \\
r\end{array}$ & & GLO & $\begin{array}{l}\text { techno } \\
\text { sphere }\end{array}$ \\
\hline $\begin{array}{l}\text { transport, pipeline, supercritical CO2, } 200 \mathrm{~km} \mathrm{w} \\
\text { recompression }\end{array}$ & & $\begin{array}{r}1.30 \\
E+03 \\
\end{array}$ & $\begin{array}{l}\text { ton } \\
\text { kilomete } \\
r\end{array}$ & & RER & $\begin{array}{l}\text { techno } \\
\text { sphere }\end{array}$ \\
\hline $\begin{array}{l}\text { transport, pipeline, supercritical CO2, 200km w/o } \\
\text { recompression }\end{array}$ & & $\begin{array}{r}2.00 \\
E+02\end{array}$ & $\begin{array}{l}\text { ton } \\
\text { kilomete } \\
r\end{array}$ & & RER & $\begin{array}{l}\text { techno } \\
\text { sphere }\end{array}$ \\
\hline carbon dioxide, captured from atmosphere, $\mathrm{HTHP}+\mathrm{Grid}, \mathrm{CL}$ & & & ton & & $\mathrm{CL}$ & \\
\hline Name & Reference Product & $\begin{array}{l}\text { Amo } \\
\text { unt }\end{array}$ & Unit & Categories & $\begin{array}{l}\text { Loca } \\
\text { tion }\end{array}$ & Type \\
\hline Carbon dioxide, fossil & & $\begin{array}{r}2.10 \\
E+01 \\
\end{array}$ & kilogram & $\begin{array}{l}\text { air:urban air } \\
\text { close to ground }\end{array}$ & & $\begin{array}{l}\text { biosph } \\
\text { ere }\end{array}$ \\
\hline carbon dioxide capture system & & $\begin{array}{l}5.00 \\
\mathrm{E}-07\end{array}$ & unit & & RER & $\begin{array}{l}\text { techno } \\
\text { sphere }\end{array}$ \\
\hline drilling, deep borehole/m & & $\begin{array}{l}3.38 \\
\mathrm{E}-05 \\
\end{array}$ & meter & & RER & $\begin{array}{l}\text { techno } \\
\text { sphere }\end{array}$ \\
\hline end of life, carbon dioxide capture system & & $\begin{array}{l}5.00 \\
\mathrm{E}-07 \\
\end{array}$ & unit & & RoW & $\begin{array}{l}\text { techno } \\
\text { sphere }\end{array}$ \\
\hline heat pump production, brine-water, $\mathrm{CO} 2$ refrigerant, $10 \mathrm{~kW}$ & & $\begin{array}{l}8.56 \\
\mathrm{E}-04 \\
\end{array}$ & unit & & $\mathrm{CH}$ & $\begin{array}{l}\text { techno } \\
\text { sphere }\end{array}$ \\
\hline market for air compressor, screw-type compressor, 300kW & $\begin{array}{l}\text { air compressor, screw-type } \\
\text { compressor, } 300 \mathrm{~kW}\end{array}$ & $\begin{array}{l}4.35 \\
\mathrm{E}-06\end{array}$ & unit & & GLO & $\begin{array}{l}\text { techno } \\
\text { sphere }\end{array}$ \\
\hline market for electricity, low voltage & electricity, low voltage & $\begin{array}{r}5.00 \\
E+02 \\
\end{array}$ & $\begin{array}{l}\text { kilowatt } \\
\text { hour }\end{array}$ & & $\mathrm{CL}$ & $\begin{array}{l}\text { techno } \\
\text { sphere }\end{array}$ \\
\hline market for electricity, low voltage & electricity, low voltage & $\begin{aligned} 1.14 \\
\mathrm{E}+02 \\
\end{aligned}$ & $\begin{array}{l}\text { kilowatt } \\
\text { hour }\end{array}$ & & $\mathrm{CL}$ & $\begin{array}{l}\text { techno } \\
\text { sphere }\end{array}$ \\
\hline market for electricity, low voltage & electricity, low voltage & $\begin{array}{r}5.17 \\
E+02 \\
\end{array}$ & $\begin{array}{l}\text { kilowatt } \\
\text { hour }\end{array}$ & & $\mathrm{CL}$ & $\begin{array}{l}\text { techno } \\
\text { sphere }\end{array}$ \\
\hline market for gas turbine, $10 \mathrm{MW}$ electrical & $\begin{array}{l}\text { gas turbine, 10MW } \\
\text { electrical }\end{array}$ & $\begin{array}{ll}2.54 \\
\mathrm{E}-08 \\
\end{array}$ & unit & & GLO & $\begin{array}{l}\text { techno } \\
\text { sphere }\end{array}$ \\
\hline market group for electricity, low voltage & electricity, low voltage & $\begin{array}{r}2.42 \\
\mathrm{E}+01 \\
\end{array}$ & $\begin{array}{l}\text { kilowatt } \\
\text { hour }\end{array}$ & & $\mathrm{BR}$ & $\begin{array}{l}\text { techno } \\
\text { sphere }\end{array}$ \\
\hline sorbent, generic & & $\begin{array}{r}3.00 \\
E+00\end{array}$ & kilogram & & RER & $\begin{array}{l}\text { techno } \\
\text { sphere }\end{array}$ \\
\hline transport, freight, lorry $>32$ metric ton, EURO 6 & $\begin{array}{l}\text { transport, freight, lorry } \\
>32 \text { metric ton, EURO6 }\end{array}$ & $\begin{array}{l}2.53 \\
\mathrm{E}-01 \\
\end{array}$ & $\begin{array}{l}\text { ton } \\
\text { kilomete } \\
r\end{array}$ & & RoW & $\begin{array}{l}\text { techno } \\
\text { sphere }\end{array}$ \\
\hline transport, freight, sea, container ship & $\begin{array}{l}\text { transport, freight, sea, } \\
\text { container ship }\end{array}$ & $\begin{array}{r}8.21 \\
E+00\end{array}$ & $\begin{array}{l}\text { ton } \\
\text { kilomete } \\
r\end{array}$ & & GLO & $\begin{array}{l}\text { techno } \\
\text { sphere }\end{array}$ \\
\hline transport, passengers, passenger aircraft, medium haul & $\begin{array}{l}\text { transport, passengers, } \\
\text { aircraft, medium haul }\end{array}$ & $\begin{array}{r}1.02 \\
E+00\end{array}$ & $\begin{array}{l}\text { person } \\
\text { kilomete } \\
r\end{array}$ & & GLO & $\begin{array}{l}\text { techno } \\
\text { sphere }\end{array}$ \\
\hline $\begin{array}{l}\text { transport, pipeline, supercritical CO2, } 200 \mathrm{~km} \mathrm{w} \\
\text { recompression }\end{array}$ & & $\begin{array}{r}1.30 \\
E+03\end{array}$ & $\begin{array}{l}\text { ton } \\
\text { kilomete } \\
r\end{array}$ & & RER & $\begin{array}{l}\text { techno } \\
\text { sphere }\end{array}$ \\
\hline $\begin{array}{l}\text { transport, pipeline, supercritical CO2, } 200 \mathrm{~km} \text { w/o } \\
\text { recompression }\end{array}$ & & $\begin{array}{r}2.00 \\
E+02 \\
\end{array}$ & $\begin{array}{l}\text { ton } \\
\text { kilomete } \\
r\end{array}$ & & RER & $\begin{array}{l}\text { techno } \\
\text { sphere }\end{array}$ \\
\hline $\begin{array}{l}\text { carbon dioxide, captured from atmosphere, Autonomous } \\
\text { Fresnel }+\mathrm{PV}, \mathrm{CL}\end{array}$ & & & ton & & $\mathrm{CL}$ & \\
\hline Name & Reference Product & $\begin{array}{l}\text { Amo } \\
\text { unt }\end{array}$ & Unit & Categories & $\begin{array}{l}\text { Loca } \\
\text { tion }\end{array}$ & Type \\
\hline Battery management system, kWh & & $\begin{array}{l}1.04 \\
E-01\end{array}$ & $\begin{array}{l}\text { kilowatt } \\
\text { hour }\end{array}$ & & GLO & $\begin{array}{l}\text { techno } \\
\text { sphere }\end{array}$ \\
\hline
\end{tabular}




\begin{tabular}{|c|c|c|c|c|c|c|}
\hline Carbon dioxide, fossil & & $\begin{array}{r}2.10 \\
E+01 \\
\end{array}$ & kilogram & $\begin{array}{l}\text { air:urban air } \\
\text { close to ground }\end{array}$ & & $\begin{array}{l}\text { biosph } \\
\text { ere }\end{array}$ \\
\hline Energy management system, kWh & & $\begin{array}{l}1.04 \\
\mathrm{E}-01 \\
\end{array}$ & $\begin{array}{l}\text { kilowatt } \\
\text { hour }\end{array}$ & & GLO & $\begin{array}{l}\text { techno } \\
\text { sphere }\end{array}$ \\
\hline Li-ion (NMC) & & $\begin{array}{l}1.04 \\
\mathrm{E}-01\end{array}$ & $\begin{array}{l}\text { kilowatt } \\
\text { hour }\end{array}$ & & GLO & $\begin{array}{l}\text { techno } \\
\text { sphere }\end{array}$ \\
\hline Power conditioning system, container system & & $\begin{array}{l}3.13 \\
\mathrm{E}-02\end{array}$ & kW & & GLO & $\begin{array}{l}\text { techno } \\
\text { sphere }\end{array}$ \\
\hline carbon dioxide capture system & & $\begin{array}{l}5.00 \\
\mathrm{E}-07 \\
\end{array}$ & unit & & RER & $\begin{array}{l}\text { techno } \\
\text { sphere }\end{array}$ \\
\hline drilling, deep borehole/m & & $\begin{array}{l}3.38 \\
\mathrm{E}-05 \\
\end{array}$ & meter & & RER & $\begin{array}{l}\text { techno } \\
\text { sphere }\end{array}$ \\
\hline $\begin{array}{l}\text { electricity production, photovoltaic, } 570 \mathrm{kWp} \text { open ground } \\
\text { installation, multi-Si }\end{array}$ & electricity, low voltage & $\begin{array}{r}5.62 \\
E+02 \\
\end{array}$ & $\begin{array}{l}\text { kilowatt } \\
\text { hour }\end{array}$ & & $\mathrm{CL}$ & $\begin{array}{l}\text { techno } \\
\text { sphere }\end{array}$ \\
\hline $\begin{array}{l}\text { electricity production, photovoltaic, } 570 \mathrm{kWp} \text { open ground } \\
\text { installation, multi-Si }\end{array}$ & electricity, low voltage & $\begin{array}{r}1.29 \\
\mathrm{E}+02 \\
\end{array}$ & $\begin{array}{l}\text { kilowatt } \\
\text { hour }\end{array}$ & & $\mathrm{CL}$ & $\begin{array}{l}\text { techno } \\
\text { sphere }\end{array}$ \\
\hline end of life, carbon dioxide capture system & & $\begin{array}{l}5.00 \\
\mathrm{E}-07 \\
\end{array}$ & unit & & RoW & $\begin{array}{l}\text { techno } \\
\text { sphere }\end{array}$ \\
\hline $\begin{array}{l}\text { heat production, Fresnel solar collector plant, Antofagasto, } \\
\text { Chile }\end{array}$ & $\begin{array}{l}\text { heat, central or small- } \\
\text { scale, Fresnel plant }\end{array}$ & $\begin{array}{r}5.40 \\
E+03 \\
\end{array}$ & $\begin{array}{l}\text { megajoul } \\
\mathrm{e}\end{array}$ & & $\mathrm{CL}$ & $\begin{array}{l}\text { techno } \\
\text { sphere }\end{array}$ \\
\hline market for air compressor, screw-type compressor, 300kW & $\begin{array}{l}\text { air compressor, screw-type } \\
\text { compressor, } 300 \mathrm{~kW}\end{array}$ & $\begin{array}{l}4.35 \\
\mathrm{E}-06 \\
\end{array}$ & unit & & GLO & $\begin{array}{l}\text { techno } \\
\text { sphere }\end{array}$ \\
\hline market for gas turbine, $10 \mathrm{MW}$ electrical & $\begin{array}{l}\text { gas turbine, } 10 \mathrm{MW} \\
\text { electrical }\end{array}$ & $\begin{array}{ll}2.54 \\
\mathrm{E}-08 \\
\end{array}$ & unit & & GLO & $\begin{array}{l}\text { techno } \\
\text { sphere }\end{array}$ \\
\hline market group for electricity, low voltage & electricity, low voltage & $\begin{array}{r}2.42 \\
\mathrm{E}+01 \\
\end{array}$ & $\begin{array}{l}\text { kilowatt } \\
\text { hour }\end{array}$ & & BR & $\begin{array}{l}\text { techno } \\
\text { sphere }\end{array}$ \\
\hline sorbent, generic & & $\begin{array}{r}3.00 \\
E+00 \\
\end{array}$ & kilogram & & RER & $\begin{array}{l}\text { techno } \\
\text { sphere }\end{array}$ \\
\hline storage unit production, Fresnel plant, Jordan, $12 \mathrm{~h}$ storage & $\begin{array}{l}\text { storage unit, Fresnel plant, } \\
\text { Jordan, } 12 \mathrm{~h} \text { storage }\end{array}$ & $\begin{array}{l}4.00 \\
E-07\end{array}$ & unit & & JO & $\begin{array}{l}\text { techno } \\
\text { sphere }\end{array}$ \\
\hline transport, freight, lorry >32 metric ton, EURO6 & $\begin{array}{l}\text { transport, freight, lorry } \\
>32 \text { metric ton, EURO6 }\end{array}$ & $\begin{array}{l}2.53 \\
\mathrm{E}-01 \\
\end{array}$ & $\begin{array}{l}\text { ton } \\
\text { kilomete } \\
\mathrm{r}\end{array}$ & & RoW & $\begin{array}{l}\text { techno } \\
\text { sphere }\end{array}$ \\
\hline transport, freight, sea, container ship & $\begin{array}{l}\text { transport, freight, sea, } \\
\text { container ship }\end{array}$ & $\begin{array}{r}8.21 \\
E+00 \\
\end{array}$ & $\begin{array}{l}\text { ton } \\
\text { kilomete } \\
r\end{array}$ & & GLO & $\begin{array}{l}\text { techno } \\
\text { sphere }\end{array}$ \\
\hline transport, passengers, passenger aircraft, medium haul & $\begin{array}{l}\text { transport, passengers, } \\
\text { aircraft, medium haul }\end{array}$ & $\begin{array}{r}1.02 \\
\mathrm{E}+00 \\
\end{array}$ & $\begin{array}{l}\text { person } \\
\text { kilomete } \\
r\end{array}$ & & GLO & $\begin{array}{l}\text { techno } \\
\text { sphere }\end{array}$ \\
\hline $\begin{array}{l}\text { transport, pipeline, supercritical CO2, } 200 \mathrm{~km} \mathrm{w} \\
\text { recompression }\end{array}$ & & $\begin{array}{r}1.30 \\
E+03 \\
\end{array}$ & $\begin{array}{l}\text { ton } \\
\text { kilomete } \\
r\end{array}$ & & RER & $\begin{array}{l}\text { techno } \\
\text { sphere }\end{array}$ \\
\hline $\begin{array}{l}\text { transport, pipeline, supercritical CO2, 200km w/o } \\
\text { recompression }\end{array}$ & & $\begin{array}{r}2.00 \\
\mathrm{E}+02 \\
\end{array}$ & $\begin{array}{l}\text { ton } \\
\text { kilomete } \\
r\end{array}$ & & RER & $\begin{array}{l}\text { techno } \\
\text { sphere }\end{array}$ \\
\hline $\begin{array}{l}\text { carbon dioxide, captured from atmosphere, Autonomous } \\
\mathrm{HTHP}+\mathrm{PV}, \mathrm{CL}\end{array}$ & & & ton & & $\mathrm{CL}$ & \\
\hline Name & Reference Product & $\begin{array}{l}\text { Amo } \\
\text { unt }\end{array}$ & Unit & Categories & $\begin{array}{l}\text { Loca } \\
\text { tion }\end{array}$ & Type \\
\hline Battery management system, $\mathrm{kWh}$ & & $\begin{array}{l}1.84 \\
\mathrm{E}-01 \\
\end{array}$ & $\begin{array}{l}\text { kilowatt } \\
\text { hour }\end{array}$ & & GLO & $\begin{array}{l}\text { techno } \\
\text { sphere }\end{array}$ \\
\hline Carbon dioxide, fossil & & $\begin{array}{r}2.10 \\
E+01 \\
\end{array}$ & kilogram & $\begin{array}{l}\text { air:urban air } \\
\text { close to ground }\end{array}$ & & $\begin{array}{l}\text { biosph } \\
\text { ere }\end{array}$ \\
\hline Energy management system, kWh & & $\begin{array}{ll}1.84 \\
\mathrm{E}-01 \\
\end{array}$ & $\begin{array}{l}\text { kilowatt } \\
\text { hour }\end{array}$ & & GLO & $\begin{array}{l}\text { techno } \\
\text { sphere }\end{array}$ \\
\hline Li-ion (NMC) & & $\begin{array}{l}1.84 \\
\mathrm{E}-01 \\
\end{array}$ & $\begin{array}{l}\text { kilowatt } \\
\text { hour }\end{array}$ & & GLO & $\begin{array}{l}\text { techno } \\
\text { sphere }\end{array}$ \\
\hline Power conditioning system, container system & & $\begin{array}{ll}5.52 \\
\mathrm{E}-02 \\
\end{array}$ & $\mathrm{~kW}$ & & GLO & $\begin{array}{l}\text { techno } \\
\text { sphere }\end{array}$ \\
\hline carbon dioxide capture system & & $\begin{array}{l}5.00 \\
\mathrm{E}-07 \\
\end{array}$ & unit & & RER & $\begin{array}{l}\text { techno } \\
\text { sphere }\end{array}$ \\
\hline drilling, deep borehole/m & & $\begin{array}{ll}3.38 \\
\mathrm{E}-05 \\
\end{array}$ & meter & & RER & $\begin{array}{l}\text { techno } \\
\text { sphere }\end{array}$ \\
\hline $\begin{array}{l}\text { electricity production, photovoltaic, } 570 \mathrm{kWp} \text { open ground } \\
\text { installation, multi-Si }\end{array}$ & electricity, low voltage & $\begin{aligned} 5.62 \\
\mathrm{E}+02 \\
\end{aligned}$ & $\begin{array}{l}\text { kilowatt } \\
\text { hour }\end{array}$ & & $\mathrm{CL}$ & $\begin{array}{l}\text { techno } \\
\text { sphere }\end{array}$ \\
\hline $\begin{array}{l}\text { electricity production, photovoltaic, } 570 \mathrm{kWp} \text { open ground } \\
\text { installation, multi-Si }\end{array}$ & electricity, low voltage & $\begin{array}{r}1.29 \\
\mathrm{E}+02 \\
\end{array}$ & $\begin{array}{l}\text { kilowatt } \\
\text { hour }\end{array}$ & & $\mathrm{CL}$ & $\begin{array}{l}\text { techno } \\
\text { sphere }\end{array}$ \\
\hline $\begin{array}{l}\text { electricity production, photovoltaic, } 570 \mathrm{kWp} \text { open ground } \\
\text { installation, multi-Si }\end{array}$ & electricity, low voltage & $\begin{array}{r}5.81 \\
\mathrm{E}+02 \\
\end{array}$ & $\begin{array}{l}\text { kilowatt } \\
\text { hour }\end{array}$ & & $\mathrm{CL}$ & $\begin{array}{l}\text { techno } \\
\text { sphere }\end{array}$ \\
\hline end of life, carbon dioxide capture system & & $\begin{array}{l}5.00 \\
\mathrm{E}-07 \\
\end{array}$ & unit & & RoW & $\begin{array}{l}\text { techno } \\
\text { sphere }\end{array}$ \\
\hline heat pump production, brine-water, $\mathrm{CO} 2$ refrigerant, $10 \mathrm{~kW}$ & & $\begin{array}{l}8.56 \\
\mathrm{E}-04\end{array}$ & unit & & $\mathrm{CH}$ & $\begin{array}{l}\text { techno } \\
\text { sphere }\end{array}$ \\
\hline market for air compressor, screw-type compressor, 300kW & $\begin{array}{l}\text { air compressor, screw-type } \\
\text { compressor, } 300 \mathrm{~kW}\end{array}$ & $\begin{array}{l}4.35 \\
\mathrm{E}-06 \\
\end{array}$ & unit & & GLO & $\begin{array}{l}\text { techno } \\
\text { sphere }\end{array}$ \\
\hline market for gas turbine, $10 \mathrm{MW}$ electrical & $\begin{array}{l}\text { gas turbine, } 10 \mathrm{MW} \\
\text { electrical }\end{array}$ & $\begin{array}{l}2.54 \\
E-08 \\
\end{array}$ & unit & & GLO & $\begin{array}{l}\text { techno } \\
\text { sphere }\end{array}$ \\
\hline
\end{tabular}




\begin{tabular}{|c|c|c|c|c|c|c|}
\hline market group for electricity, low voltage & electricity, low voltage & $\begin{array}{r}2.42 \\
E+01\end{array}$ & $\begin{array}{l}\text { kilowatt } \\
\text { hour }\end{array}$ & & $\mathrm{BR}$ & $\begin{array}{l}\text { techno } \\
\text { sphere }\end{array}$ \\
\hline sorbent, generic & & $\begin{array}{r}3.00 \\
E+00\end{array}$ & kilogram & & RER & $\begin{array}{l}\text { techno } \\
\text { sphere }\end{array}$ \\
\hline transport, freight, lorry $>32$ metric ton, EURO 6 & $\begin{array}{l}\text { transport, freight, lorry } \\
>32 \text { metric ton, EURO6 }\end{array}$ & $\begin{array}{l}2.53 \\
\mathrm{E}-01\end{array}$ & $\begin{array}{l}\text { ton } \\
\text { kilomete } \\
r\end{array}$ & & RoW & $\begin{array}{l}\text { techno } \\
\text { sphere }\end{array}$ \\
\hline transport, freight, sea, container ship & $\begin{array}{l}\text { transport, freight, sea, } \\
\text { container ship }\end{array}$ & $\begin{array}{r}8.21 \\
\mathrm{E}+00 \\
\end{array}$ & $\begin{array}{l}\text { ton } \\
\text { kilomete } \\
\mathrm{r}\end{array}$ & & GLO & $\begin{array}{l}\text { techno } \\
\text { sphere }\end{array}$ \\
\hline transport, passengers, passenger aircraft, medium haul & $\begin{array}{l}\text { transport, passengers, } \\
\text { aircraft, medium haul }\end{array}$ & $\begin{array}{r}1.02 \\
E+00 \\
\end{array}$ & $\begin{array}{l}\text { person } \\
\text { kilomete } \\
r\end{array}$ & & GLO & $\begin{array}{l}\text { techno } \\
\text { sphere }\end{array}$ \\
\hline $\begin{array}{l}\text { transport, pipeline, supercritical CO2, } 200 \mathrm{~km} \mathrm{w} \\
\text { recompression }\end{array}$ & & $\begin{array}{r}1.30 \\
\mathrm{E}+03\end{array}$ & $\begin{array}{l}\text { ton } \\
\text { kilomete } \\
r\end{array}$ & & RER & $\begin{array}{l}\text { techno } \\
\text { sphere }\end{array}$ \\
\hline $\begin{array}{l}\text { transport, pipeline, supercritical } \mathrm{CO} 2,200 \mathrm{~km} \text { w/o } \\
\text { recompression }\end{array}$ & & $\begin{array}{r}2.00 \\
E+02\end{array}$ & $\begin{array}{l}\text { ton } \\
\text { kilomete } \\
r\end{array}$ & & RER & $\begin{array}{l}\text { techno } \\
\text { sphere }\end{array}$ \\
\hline $\begin{array}{l}\text { carbon dioxide, captured from atmosphere, Waste heat + } \\
\text { Grid, NO }\end{array}$ & & & ton & & NO & \\
\hline Name & Reference Product & $\begin{array}{l}\text { Amo } \\
\text { unt }\end{array}$ & Unit & Categories & $\begin{array}{l}\text { Loca } \\
\text { tion }\end{array}$ & Type \\
\hline Carbon dioxide, fossil & & $\begin{array}{r}1.40 \\
E+00 \\
\end{array}$ & kilogram & $\begin{array}{l}\text { air:urban air } \\
\text { close to ground }\end{array}$ & & $\begin{array}{l}\text { biosph } \\
\text { ere }\end{array}$ \\
\hline carbon dioxide capture system & & $\begin{array}{l}5.00 \\
\mathrm{E}-07 \\
\end{array}$ & unit & & RER & $\begin{array}{l}\text { techno } \\
\text { sphere }\end{array}$ \\
\hline drilling, deep borehole/m & & $\begin{array}{ll}3.38 \\
\mathrm{E}-05 \\
\end{array}$ & meter & & RER & $\begin{array}{l}\text { techno } \\
\text { sphere }\end{array}$ \\
\hline end of life, carbon dioxide capture system & & $\begin{array}{l}5.00 \\
\mathrm{E}-07 \\
\end{array}$ & unit & & RoW & $\begin{array}{l}\text { techno } \\
\text { sphere }\end{array}$ \\
\hline $\begin{array}{l}\text { heat, from municipal waste incineration to generic market for } \\
\text { heat district or industrial, other than natural gas }\end{array}$ & $\begin{array}{l}\text { heat, district or industrial, } \\
\text { other than natural gas }\end{array}$ & $\begin{array}{r}5.40 \\
E+03 \\
\end{array}$ & $\begin{array}{l}\text { megajoul } \\
\mathrm{e}\end{array}$ & & NO & $\begin{array}{l}\text { techno } \\
\text { sphere }\end{array}$ \\
\hline market for air compressor, screw-type compressor, $300 \mathrm{~kW}$ & $\begin{array}{l}\text { air compressor, screw-type } \\
\text { compressor, 300kW }\end{array}$ & $\begin{array}{l}4.35 \\
\mathrm{E}-06 \\
\end{array}$ & unit & & GLO & $\begin{array}{l}\text { techno } \\
\text { sphere }\end{array}$ \\
\hline market for electricity, low voltage & electricity, low voltage & $\begin{array}{r}5.00 \\
E+02 \\
\end{array}$ & $\begin{array}{l}\text { kilowatt } \\
\text { hour }\end{array}$ & & NO & $\begin{array}{l}\text { techno } \\
\text { sphere }\end{array}$ \\
\hline market for electricity, low voltage & electricity, low voltage & $\begin{array}{r}1.14 \\
E+02 \\
\end{array}$ & $\begin{array}{l}\text { kilowatt } \\
\text { hour }\end{array}$ & & NO & $\begin{array}{l}\text { techno } \\
\text { sphere }\end{array}$ \\
\hline market for electricity, low voltage & electricity, low voltage & $\begin{array}{r}2.42 \\
E+01 \\
\end{array}$ & $\begin{array}{l}\text { kilowatt } \\
\text { hour }\end{array}$ & & NO & $\begin{array}{l}\text { techno } \\
\text { sphere }\end{array}$ \\
\hline market for gas turbine, $10 \mathrm{MW}$ electrical & $\begin{array}{l}\text { gas turbine, } 10 \mathrm{MW} \\
\text { electrical }\end{array}$ & $\begin{array}{l}2.54 \\
\mathrm{E}-08 \\
\end{array}$ & unit & & GLO & $\begin{array}{l}\text { techno } \\
\text { sphere }\end{array}$ \\
\hline sorbent, generic & & $\begin{array}{r}3.00 \\
E+00 \\
\end{array}$ & kilogram & & RER & $\begin{array}{l}\text { techno } \\
\text { sphere }\end{array}$ \\
\hline transport, freight, lorry $>32$ metric ton, EURO 6 & $\begin{array}{l}\text { transport, freight, lorry } \\
>32 \text { metric ton, EURO6 }\end{array}$ & $\begin{array}{l}2.53 \\
\mathrm{E}-01 \\
\end{array}$ & $\begin{array}{l}\text { ton } \\
\text { kilomete } \\
r\end{array}$ & & RoW & $\begin{array}{l}\text { techno } \\
\text { sphere }\end{array}$ \\
\hline transport, freight, sea, container ship & $\begin{array}{l}\text { transport, freight, sea, } \\
\text { container ship }\end{array}$ & $\begin{array}{r}3.04 \\
E+00 \\
\end{array}$ & $\begin{array}{l}\text { ton } \\
\text { kilomete } \\
r\end{array}$ & & GLO & $\begin{array}{l}\text { techno } \\
\text { sphere }\end{array}$ \\
\hline transport, passengers, passenger aircraft, medium haul & $\begin{array}{l}\text { transport, passengers, } \\
\text { aircraft, medium haul }\end{array}$ & $\begin{array}{l}1.48 \\
\mathrm{E}-01 \\
\end{array}$ & $\begin{array}{l}\text { person } \\
\text { kilomete } \\
r\end{array}$ & & GLO & $\begin{array}{l}\text { techno } \\
\text { sphere }\end{array}$ \\
\hline $\begin{array}{l}\text { transport, pipeline, supercritical CO2, } 200 \mathrm{~km} \mathrm{w/o} \\
\text { recompression }\end{array}$ & & $\begin{array}{r}1.00 \\
E+02\end{array}$ & $\begin{array}{l}\text { ton } \\
\text { kilomete } \\
r\end{array}$ & & RER & $\begin{array}{l}\text { techno } \\
\text { sphere }\end{array}$ \\
\hline $\begin{array}{l}\text { carbon dioxide, captured from atmosphere, HTHP + Grid, } \\
\text { NO }\end{array}$ & & & ton & & NO & \\
\hline Name & Reference Product & $\begin{array}{l}\text { Amo } \\
\text { unt }\end{array}$ & Unit & Categories & $\begin{array}{l}\text { Loca } \\
\text { tion }\end{array}$ & Type \\
\hline Carbon dioxide, fossil & & $\begin{array}{r}1.40 \\
E+00 \\
\end{array}$ & kilogram & $\begin{array}{l}\text { air:urban air } \\
\text { close to ground }\end{array}$ & & $\begin{array}{l}\text { biosph } \\
\text { ere }\end{array}$ \\
\hline carbon dioxide capture system & & $\begin{array}{l}5.00 \\
\mathrm{E}-07 \\
\end{array}$ & unit & & RER & $\begin{array}{l}\text { techno } \\
\text { sphere }\end{array}$ \\
\hline drilling, deep borehole/m & & $\begin{array}{ll}3.38 \\
\mathrm{E}-05 \\
\end{array}$ & meter & & RER & $\begin{array}{l}\text { techno } \\
\text { sphere }\end{array}$ \\
\hline end of life, carbon dioxide capture system & & $\begin{array}{l}5.00 \\
\mathrm{E}-07\end{array}$ & unit & & RoW & $\begin{array}{l}\text { techno } \\
\text { sphere }\end{array}$ \\
\hline heat pump production, brine-water, $\mathrm{CO} 2$ refrigerant, $10 \mathrm{~kW}$ & & $\begin{array}{l}8.56 \\
\mathrm{E}-04 \\
\end{array}$ & unit & & $\mathrm{CH}$ & $\begin{array}{l}\text { techno } \\
\text { sphere }\end{array}$ \\
\hline market for air compressor, screw-type compressor, $300 \mathrm{~kW}$ & $\begin{array}{l}\text { air compressor, screw-type } \\
\text { compressor, } 300 \mathrm{~kW}\end{array}$ & $\begin{array}{l}4.35 \\
\mathrm{E}-06 \\
\end{array}$ & unit & & GLO & $\begin{array}{l}\text { techno } \\
\text { sphere }\end{array}$ \\
\hline market for electricity, low voltage & electricity, low voltage & $\begin{array}{r}5.00 \\
E+02 \\
\end{array}$ & $\begin{array}{l}\text { kilowatt } \\
\text { hour }\end{array}$ & & NO & $\begin{array}{l}\text { techno } \\
\text { sphere }\end{array}$ \\
\hline
\end{tabular}




\begin{tabular}{|c|c|c|c|c|c|c|}
\hline market for electricity, low voltage & electricity, low voltage & $\begin{array}{r}1.14 \\
\mathrm{E}+02\end{array}$ & $\begin{array}{l}\text { kilowatt } \\
\text { hour }\end{array}$ & & NO & $\begin{array}{l}\text { techno } \\
\text { sphere }\end{array}$ \\
\hline market for electricity, low voltage & electricity, low voltage & $\begin{array}{r}5.17 \\
\mathrm{E}+02 \\
\end{array}$ & $\begin{array}{l}\text { kilowatt } \\
\text { hour }\end{array}$ & & NO & $\begin{array}{l}\text { techno } \\
\text { sphere }\end{array}$ \\
\hline market for electricity, low voltage & electricity, low voltage & $\begin{array}{r}2.42 \\
E+01\end{array}$ & $\begin{array}{l}\text { kilowatt } \\
\text { hour }\end{array}$ & & NO & $\begin{array}{l}\text { techno } \\
\text { sphere }\end{array}$ \\
\hline market for gas turbine, $10 \mathrm{MW}$ electrical & $\begin{array}{l}\text { gas turbine, } 10 \mathrm{MW} \\
\text { electrical }\end{array}$ & $\begin{array}{l}2.54 \\
\mathrm{E}-08 \\
\end{array}$ & unit & & GLO & $\begin{array}{l}\text { techno } \\
\text { sphere }\end{array}$ \\
\hline sorbent, generic & & $\begin{array}{r}3.00 \\
\mathrm{E}+00 \\
\end{array}$ & kilogram & & RER & $\begin{array}{l}\text { techno } \\
\text { sphere }\end{array}$ \\
\hline transport, freight, lorry >32 metric ton, EURO6 & $\begin{array}{l}\text { transport, freight, lorry } \\
>32 \text { metric ton, EURO6 }\end{array}$ & $\begin{array}{l}2.53 \\
\mathrm{E}-01 \\
\end{array}$ & $\begin{array}{l}\text { ton } \\
\text { kilomete } \\
r\end{array}$ & & RoW & $\begin{array}{l}\text { techno } \\
\text { sphere }\end{array}$ \\
\hline transport, freight, sea, container ship & $\begin{array}{l}\text { transport, freight, sea, } \\
\text { container ship }\end{array}$ & $\begin{array}{r}3.04 \\
\mathrm{E}+00 \\
\end{array}$ & $\begin{array}{l}\text { ton } \\
\text { kilomete } \\
r\end{array}$ & & GLO & $\begin{array}{l}\text { techno } \\
\text { sphere }\end{array}$ \\
\hline transport, passengers, passenger aircraft, medium haul & $\begin{array}{l}\text { transport, passengers, } \\
\text { aircraft, medium haul }\end{array}$ & $\begin{array}{l}1.48 \\
\mathrm{E}-01 \\
\end{array}$ & $\begin{array}{l}\text { person } \\
\text { kilomete } \\
r\end{array}$ & & GLO & $\begin{array}{l}\text { techno } \\
\text { sphere }\end{array}$ \\
\hline $\begin{array}{l}\text { transport, pipeline, supercritical CO2, 200km w/o } \\
\text { recompression }\end{array}$ & & $\begin{array}{r}1.00 \\
E+02 \\
\end{array}$ & $\begin{array}{l}\text { ton } \\
\text { kilomete } \\
r\end{array}$ & & RER & $\begin{array}{l}\text { techno } \\
\text { sphere }\end{array}$ \\
\hline $\begin{array}{l}\text { carbon dioxide, captured from atmosphere, Waste heat + } \\
\text { Grid, GR }\end{array}$ & & & ton & & GR & \\
\hline Name & Reference Product & $\begin{array}{l}\text { Amo } \\
\text { unt }\end{array}$ & Unit & Categories & $\begin{array}{l}\text { Loca } \\
\text { tion }\end{array}$ & Type \\
\hline Carbon dioxide, fossil & & $\begin{array}{r}7.00 \\
E+00 \\
\end{array}$ & kilogram & $\begin{array}{l}\text { air:urban air } \\
\text { close to ground }\end{array}$ & & $\begin{array}{l}\text { biosph } \\
\text { ere }\end{array}$ \\
\hline carbon dioxide capture system & & $\begin{array}{l}5.00 \\
\mathrm{E}-07 \\
\end{array}$ & unit & & RER & $\begin{array}{l}\text { techno } \\
\text { sphere }\end{array}$ \\
\hline drilling, deep borehole/m & & $\begin{array}{ll}3.38 \\
\mathrm{E}-05 \\
\end{array}$ & meter & & RER & $\begin{array}{l}\text { techno } \\
\text { sphere }\end{array}$ \\
\hline end of life, carbon dioxide capture system & & $\begin{array}{ll}5.00 \\
\mathrm{E}-07 \\
\end{array}$ & unit & & RoW & $\begin{array}{l}\text { techno } \\
\text { sphere }\end{array}$ \\
\hline $\begin{array}{l}\text { heat, from municipal waste incineration to generic market for } \\
\text { heat district or industrial, other than natural gas }\end{array}$ & $\begin{array}{l}\text { heat, district or industrial, } \\
\text { other than natural gas }\end{array}$ & $\begin{array}{r}5.40 \\
\mathrm{E}+03 \\
\end{array}$ & $\begin{array}{l}\text { megajoul } \\
\mathrm{e}\end{array}$ & & RoW & $\begin{array}{l}\text { techno } \\
\text { sphere }\end{array}$ \\
\hline market for air compressor, screw-type compressor, 300kW & $\begin{array}{l}\text { air compressor, screw-type } \\
\text { compressor, } 300 \mathrm{~kW}\end{array}$ & $\begin{array}{l}4.35 \\
\mathrm{E}-06 \\
\end{array}$ & unit & & GLO & $\begin{array}{l}\text { techno } \\
\text { sphere }\end{array}$ \\
\hline market for electricity, low voltage & electricity, low voltage & $\begin{array}{r}5.00 \\
E+02 \\
\end{array}$ & $\begin{array}{l}\text { kilowatt } \\
\text { hour }\end{array}$ & & GR & $\begin{array}{l}\text { techno } \\
\text { sphere }\end{array}$ \\
\hline market for electricity, low voltage & electricity, low voltage & $\begin{array}{r}1.14 \\
E+02\end{array}$ & $\begin{array}{l}\text { kilowatt } \\
\text { hour }\end{array}$ & & GR & $\begin{array}{l}\text { techno } \\
\text { sphere }\end{array}$ \\
\hline market for electricity, low voltage & electricity, low voltage & $\begin{array}{r}2.42 \\
\mathrm{E}+01 \\
\end{array}$ & $\begin{array}{l}\text { kilowatt } \\
\text { hour }\end{array}$ & & GR & $\begin{array}{l}\text { techno } \\
\text { sphere }\end{array}$ \\
\hline market for gas turbine, $10 \mathrm{MW}$ electrical & $\begin{array}{l}\text { gas turbine, } 10 \mathrm{MW} \\
\text { electrical }\end{array}$ & $\begin{array}{l}2.54 \\
\mathrm{E}-08 \\
\end{array}$ & unit & & GLO & $\begin{array}{l}\text { techno } \\
\text { sphere }\end{array}$ \\
\hline sorbent, generic & & $\begin{array}{r}3.00 \\
E+00\end{array}$ & kilogram & & RER & $\begin{array}{l}\text { techno } \\
\text { sphere }\end{array}$ \\
\hline transport, freight, lorry $>32$ metric ton, EURO 6 & $\begin{array}{l}\text { transport, freight, lorry } \\
>32 \text { metric ton, EURO6 }\end{array}$ & $\begin{array}{l}2.53 \\
\mathrm{E}-01 \\
\end{array}$ & $\begin{array}{l}\text { ton } \\
\text { kilomete } \\
r\end{array}$ & & RoW & $\begin{array}{l}\text { techno } \\
\text { sphere }\end{array}$ \\
\hline transport, freight, sea, container ship & $\begin{array}{l}\text { transport, freight, sea, } \\
\text { container ship }\end{array}$ & $\begin{array}{r}1.16 \\
\mathrm{E}+00\end{array}$ & $\begin{array}{l}\text { ton } \\
\text { kilomete } \\
r\end{array}$ & & GLO & $\begin{array}{l}\text { techno } \\
\text { sphere }\end{array}$ \\
\hline transport, passengers, passenger aircraft, medium haul & $\begin{array}{l}\text { transport, passengers, } \\
\text { aircraft, medium haul }\end{array}$ & $\begin{array}{l}2.02 \\
\mathrm{E}-01\end{array}$ & $\begin{array}{l}\text { person } \\
\text { kilomete } \\
r\end{array}$ & & GLO & $\begin{array}{l}\text { techno } \\
\text { sphere }\end{array}$ \\
\hline $\begin{array}{l}\text { transport, pipeline, supercritical CO2, } 200 \mathrm{~km} \mathrm{w} \\
\text { recompression }\end{array}$ & & $\begin{array}{r}3.00 \\
E+02 \\
\end{array}$ & $\begin{array}{l}\text { ton } \\
\text { kilomete } \\
r\end{array}$ & & RER & $\begin{array}{l}\text { techno } \\
\text { sphere }\end{array}$ \\
\hline $\begin{array}{l}\text { transport, pipeline, supercritical CO2, } 200 \mathrm{~km} \text { w/o } \\
\text { recompression }\end{array}$ & & $\begin{array}{r}2.00 \\
\mathrm{E}+02 \\
\end{array}$ & $\begin{array}{l}\text { ton } \\
\text { kilomete } \\
r\end{array}$ & & RER & $\begin{array}{l}\text { techno } \\
\text { sphere }\end{array}$ \\
\hline $\begin{array}{l}\text { carbon dioxide, captured from atmosphere, Waste heat + } \\
\text { PV + Battery, GR }\end{array}$ & & & ton & & GR & \\
\hline Name & Reference Product & $\begin{array}{l}\text { Amo } \\
\text { unt }\end{array}$ & Unit & Categories & $\begin{array}{l}\text { Loca } \\
\text { tion }\end{array}$ & Type \\
\hline Battery management system, kWh & & $\begin{array}{l}9.99 \\
\mathrm{E}-02 \\
\end{array}$ & $\begin{array}{l}\text { kilowatt } \\
\text { hour }\end{array}$ & & GLO & $\begin{array}{l}\text { techno } \\
\text { sphere }\end{array}$ \\
\hline Carbon dioxide, fossil & & $\begin{array}{r}7.00 \\
E+00 \\
\end{array}$ & kilogram & $\begin{array}{l}\text { air:urban air } \\
\text { close to ground }\end{array}$ & & $\begin{array}{l}\text { biosph } \\
\text { ere }\end{array}$ \\
\hline Energy management system, kWh & & $\begin{array}{l}9.99 \\
E-02 \\
\end{array}$ & $\begin{array}{l}\text { kilowatt } \\
\text { hour }\end{array}$ & & GLO & $\begin{array}{l}\text { techno } \\
\text { sphere }\end{array}$ \\
\hline Li-ion (NMC) & & $\begin{array}{l}9.99 \\
E-02\end{array}$ & $\begin{array}{l}\text { kilowatt } \\
\text { hour }\end{array}$ & & GLO & $\begin{array}{l}\text { techno } \\
\text { sphere }\end{array}$ \\
\hline
\end{tabular}




\begin{tabular}{|c|c|c|c|c|c|c|}
\hline Power conditioning system, container system & & $\begin{array}{l}3.00 \\
\mathrm{E}-02\end{array}$ & $\mathrm{~kW}$ & & GLO & $\begin{array}{l}\text { techno } \\
\text { sphere }\end{array}$ \\
\hline carbon dioxide capture system & & $\begin{array}{l}5.00 \\
\mathrm{E}-07\end{array}$ & unit & & RER & $\begin{array}{l}\text { techno } \\
\text { sphere }\end{array}$ \\
\hline drilling, deep borehole/m & & $\begin{array}{l}3.38 \\
\mathrm{E}-05\end{array}$ & meter & & RER & $\begin{array}{l}\text { techno } \\
\text { sphere }\end{array}$ \\
\hline $\begin{array}{l}\text { electricity production, photovoltaic, } 570 \mathrm{kWp} \text { open ground } \\
\text { installation, multi-Si }\end{array}$ & electricity, low voltage & $\begin{array}{r}5.62 \\
E+02\end{array}$ & $\begin{array}{l}\text { kilowatt } \\
\text { hour }\end{array}$ & & GR & $\begin{array}{l}\text { techno } \\
\text { sphere }\end{array}$ \\
\hline $\begin{array}{l}\text { electricity production, photovoltaic, } 570 \mathrm{kWp} \text { open ground } \\
\text { installation, multi-Si }\end{array}$ & electricity, low voltage & $\begin{array}{r}1.29 \\
E+02 \\
\end{array}$ & $\begin{array}{l}\text { kilowatt } \\
\text { hour }\end{array}$ & & $\mathrm{GR}$ & $\begin{array}{l}\text { techno } \\
\text { sphere }\end{array}$ \\
\hline end of life, carbon dioxide capture system & & $\begin{array}{ll}5.00 \\
\mathrm{E}-07 \\
\end{array}$ & unit & & RoW & $\begin{array}{l}\text { techno } \\
\text { sphere }\end{array}$ \\
\hline $\begin{array}{l}\text { heat, from municipal waste incineration to generic market for } \\
\text { heat district or industrial, other than natural gas }\end{array}$ & $\begin{array}{l}\text { heat, district or industrial, } \\
\text { other than natural gas }\end{array}$ & $\begin{array}{r}5.40 \\
\mathrm{E}+03 \\
\end{array}$ & $\begin{array}{l}\text { megajoul } \\
\mathrm{e}\end{array}$ & & RoW & $\begin{array}{l}\text { techno } \\
\text { sphere }\end{array}$ \\
\hline market for air compressor, screw-type compressor, 300kW & $\begin{array}{l}\text { air compressor, screw-type } \\
\text { compressor, } 300 \mathrm{~kW}\end{array}$ & $\begin{array}{l}4.35 \\
\mathrm{E}-06 \\
\end{array}$ & unit & & GLO & $\begin{array}{l}\text { techno } \\
\text { sphere }\end{array}$ \\
\hline market for electricity, low voltage & electricity, low voltage & $\begin{array}{r}2.42 \\
E+01 \\
\end{array}$ & $\begin{array}{l}\text { kilowatt } \\
\text { hour }\end{array}$ & & GR & $\begin{array}{l}\text { techno } \\
\text { sphere }\end{array}$ \\
\hline market for gas turbine, $10 \mathrm{MW}$ electrical & $\begin{array}{l}\text { gas turbine, } 10 \mathrm{MW} \\
\text { electrical }\end{array}$ & $\begin{array}{l}2.54 \\
\mathrm{E}-08 \\
\end{array}$ & unit & & GLO & $\begin{array}{l}\text { techno } \\
\text { sphere }\end{array}$ \\
\hline sorbent, generic & & $\begin{array}{r}3.00 \\
E+00 \\
\end{array}$ & kilogram & & RER & $\begin{array}{l}\text { techno } \\
\text { sphere }\end{array}$ \\
\hline transport, freight, lorry $>32$ metric ton, EURO 6 & $\begin{array}{l}\text { transport, freight, lorry } \\
>32 \text { metric ton, EURO6 }\end{array}$ & $\begin{array}{l}2.53 \\
\mathrm{E}-01 \\
\end{array}$ & $\begin{array}{l}\text { ton } \\
\text { kilomete } \\
\mathrm{r}\end{array}$ & & RoW & $\begin{array}{l}\text { techno } \\
\text { sphere }\end{array}$ \\
\hline transport, freight, sea, container ship & $\begin{array}{l}\text { transport, freight, sea, } \\
\text { container ship }\end{array}$ & $\begin{array}{r}1.16 \\
E+00 \\
\end{array}$ & $\begin{array}{l}\text { ton } \\
\text { kilomete } \\
\mathrm{r}\end{array}$ & & GLO & $\begin{array}{l}\text { techno } \\
\text { sphere }\end{array}$ \\
\hline transport, passengers, passenger aircraft, medium haul & $\begin{array}{l}\text { transport, passengers, } \\
\text { aircraft, medium haul }\end{array}$ & $\begin{array}{l}2.02 \\
\mathrm{E}-01\end{array}$ & $\begin{array}{l}\text { person } \\
\text { kilomete } \\
r\end{array}$ & & GLO & $\begin{array}{l}\text { techno } \\
\text { sphere }\end{array}$ \\
\hline $\begin{array}{l}\text { transport, pipeline, supercritical } \mathrm{CO} 2,200 \mathrm{~km} \mathrm{w} \\
\text { recompression }\end{array}$ & & $\begin{array}{r}3.00 \\
E+02 \\
\end{array}$ & $\begin{array}{l}\text { ton } \\
\text { kilomete } \\
\mathrm{r}\end{array}$ & & RER & $\begin{array}{l}\text { techno } \\
\text { sphere }\end{array}$ \\
\hline $\begin{array}{l}\text { transport, pipeline, supercritical CO2, } 200 \mathrm{~km} \text { w/o } \\
\text { recompression }\end{array}$ & & $\begin{array}{r}2.00 \\
E+02 \\
\end{array}$ & $\begin{array}{l}\text { ton } \\
\text { kilomete } \\
\mathrm{r}\end{array}$ & & RER & $\begin{array}{l}\text { techno } \\
\text { sphere }\end{array}$ \\
\hline carbon dioxide, captured from atmosphere, HTHP + Grid, GR & & & ton & & GR & \\
\hline e & Reference Product & $\begin{array}{l}\text { Amo } \\
\text { unt }\end{array}$ & Unit & Categories & $\begin{array}{l}\text { Loca } \\
\text { tion }\end{array}$ & Type \\
\hline Carbon dioxide, fossil & & $\begin{array}{r}7.00 \\
E+00 \\
\end{array}$ & kilogram & $\begin{array}{l}\text { air:urban air } \\
\text { close to ground }\end{array}$ & & $\begin{array}{l}\text { biosph } \\
\text { ere }\end{array}$ \\
\hline carbon dioxide capture system & & $\begin{array}{l}5.00 \\
\mathrm{E}-07 \\
\end{array}$ & unit & & RER & $\begin{array}{l}\text { techno } \\
\text { sphere }\end{array}$ \\
\hline drilling, deep borehole/m & & $\begin{array}{ll}3.38 \\
\mathrm{E}-05 \\
\end{array}$ & meter & & RER & $\begin{array}{l}\text { techno } \\
\text { sphere }\end{array}$ \\
\hline end of life, carbon dioxide capture system & & $\begin{array}{l}5.00 \\
\mathrm{E}-07 \\
\end{array}$ & unit & & RoW & $\begin{array}{l}\text { techno } \\
\text { sphere }\end{array}$ \\
\hline heat pump production, brine-water, $\mathrm{CO} 2$ refrigerant, $10 \mathrm{~kW}$ & & $\begin{array}{l}8.56 \\
\mathrm{E}-04 \\
\end{array}$ & unit & & $\mathrm{CH}$ & $\begin{array}{l}\text { techno } \\
\text { sphere }\end{array}$ \\
\hline market for air compressor, screw-type compressor, 300kW & $\begin{array}{l}\text { air compressor, screw-type } \\
\text { compressor, } 300 \mathrm{~kW}\end{array}$ & $\begin{array}{l}4.35 \\
\mathrm{E}-06 \\
\end{array}$ & unit & & GLO & $\begin{array}{l}\text { techno } \\
\text { sphere }\end{array}$ \\
\hline market for electricity, low voltage & electricity, low voltage & $\begin{array}{r}5.00 \\
E+02 \\
\end{array}$ & $\begin{array}{l}\text { kilowatt } \\
\text { hour }\end{array}$ & & GR & $\begin{array}{l}\text { techno } \\
\text { sphere }\end{array}$ \\
\hline market for electricity, low voltage & electricity, low voltage & $\begin{array}{r}1.14 \\
\mathrm{E}+02 \\
\end{array}$ & $\begin{array}{l}\text { kilowatt } \\
\text { hour }\end{array}$ & & $\mathrm{GR}$ & $\begin{array}{l}\text { techno } \\
\text { sphere }\end{array}$ \\
\hline market for electricity, low voltage & electricity, low voltage & $\begin{array}{r}5.17 \\
E+02 \\
\end{array}$ & $\begin{array}{l}\text { kilowatt } \\
\text { hour }\end{array}$ & & $\mathrm{GR}$ & $\begin{array}{l}\text { techno } \\
\text { sphere }\end{array}$ \\
\hline market for electricity, low voltage & electricity, low voltage & $\begin{array}{r}2.42 \\
E+01 \\
\end{array}$ & $\begin{array}{l}\text { kilowatt } \\
\text { hour }\end{array}$ & & $\mathrm{GR}$ & $\begin{array}{l}\text { techno } \\
\text { sphere }\end{array}$ \\
\hline market for gas turbine, $10 \mathrm{MW}$ electrical & $\begin{array}{l}\text { gas turbine, 10MW } \\
\text { electrical }\end{array}$ & $\begin{array}{l}2.54 \\
\mathrm{E}-08 \\
\end{array}$ & unit & & GLO & $\begin{array}{l}\text { techno } \\
\text { sphere }\end{array}$ \\
\hline sorbent, generic & & $\begin{array}{r}3.00 \\
E+00 \\
\end{array}$ & kilogram & & RER & $\begin{array}{l}\text { techno } \\
\text { sphere }\end{array}$ \\
\hline transport, freight, lorry $>32$ metric ton, EURO 6 & $\begin{array}{l}\text { transport, freight, lorry } \\
>32 \text { metric ton, EURO6 }\end{array}$ & $\begin{array}{l}2.53 \\
\mathrm{E}-01 \\
\end{array}$ & $\begin{array}{l}\text { ton } \\
\text { kilomete } \\
\mathrm{r} \\
\end{array}$ & & RoW & $\begin{array}{l}\text { techno } \\
\text { sphere }\end{array}$ \\
\hline transport, freight, sea, container ship & $\begin{array}{l}\text { transport, freight, sea, } \\
\text { container ship }\end{array}$ & $\begin{array}{r}1.16 \\
\mathrm{E}+00 \\
\end{array}$ & $\begin{array}{l}\text { ton } \\
\text { kilomete } \\
\mathrm{r}\end{array}$ & & GLO & $\begin{array}{l}\text { techno } \\
\text { sphere }\end{array}$ \\
\hline transport, passengers, passenger aircraft, medium haul & $\begin{array}{l}\text { transport, passengers, } \\
\text { aircraft, medium haul }\end{array}$ & $\begin{array}{l}2.02 \\
\mathrm{E}-01 \\
\end{array}$ & $\begin{array}{l}\text { person } \\
\text { kilomete } \\
r\end{array}$ & & GLO & $\begin{array}{l}\text { techno } \\
\text { sphere }\end{array}$ \\
\hline $\begin{array}{l}\text { transport, pipeline, supercritical } \mathrm{CO} 2,200 \mathrm{~km} \mathrm{w} \\
\text { recompression }\end{array}$ & & $\begin{array}{r}3.00 \\
E+02 \\
\end{array}$ & $\begin{array}{l}\text { ton } \\
\text { kilomete } \\
\mathrm{r}\end{array}$ & & RER & $\begin{array}{l}\text { techno } \\
\text { sphere }\end{array}$ \\
\hline
\end{tabular}




\begin{tabular}{|c|c|c|c|c|c|c|}
\hline $\begin{array}{l}\text { transport, pipeline, supercritical CO2, } 200 \mathrm{~km} \text { w/o } \\
\text { recompression }\end{array}$ & & $\begin{array}{r}2.00 \\
E+02\end{array}$ & $\begin{array}{l}\text { ton } \\
\text { kilomete } \\
r\end{array}$ & & RER & $\begin{array}{l}\text { techno } \\
\text { sphere }\end{array}$ \\
\hline $\begin{array}{l}\text { carbon dioxide, captured from atmosphere, Autonomous } \\
\text { Fresnel + PV, GR }\end{array}$ & & & ton & & GR & \\
\hline ( & Reference Product & $\begin{array}{l}\text { Amo } \\
\text { unt }\end{array}$ & Unit & Categories & $\begin{array}{l}\text { Loca } \\
\text { tion }\end{array}$ & Type \\
\hline Battery management system, kWh & & $\begin{array}{ll}1.04 \\
\mathrm{E}-01 \\
\end{array}$ & $\begin{array}{l}\text { kilowatt } \\
\text { hour }\end{array}$ & & GLO & $\begin{array}{l}\text { techno } \\
\text { sphere }\end{array}$ \\
\hline Carbon dioxide, fossil & & $\begin{array}{r}7.00 \\
E+00 \\
\end{array}$ & kilogram & $\begin{array}{l}\text { air:urban air } \\
\text { close to ground }\end{array}$ & & $\begin{array}{l}\text { biosph } \\
\text { ere }\end{array}$ \\
\hline Energy management system, $\mathrm{kWh}$ & & $\begin{array}{ll}1.04 \\
\mathrm{E}-01 \\
\end{array}$ & $\begin{array}{l}\text { kilowatt } \\
\text { hour }\end{array}$ & & GLO & $\begin{array}{l}\text { techno } \\
\text { sphere }\end{array}$ \\
\hline Li-ion (NMC) & & $\begin{array}{ll}1.04 \\
\mathrm{E}-01 \\
\end{array}$ & $\begin{array}{l}\text { kilowatt } \\
\text { hour }\end{array}$ & & GLO & $\begin{array}{l}\text { techno } \\
\text { sphere }\end{array}$ \\
\hline Power conditioning system, container system & & $\begin{array}{l}3.13 \\
\mathrm{E}-02 \\
\end{array}$ & kW & & GLO & $\begin{array}{l}\text { techno } \\
\text { sphere }\end{array}$ \\
\hline carbon dioxide capture system & & $\begin{array}{l}5.00 \\
\mathrm{E}-07\end{array}$ & unit & & RER & $\begin{array}{l}\text { techno } \\
\text { sphere }\end{array}$ \\
\hline drilling, deep borehole/m & & $\begin{array}{l}3.38 \\
\mathrm{E}-05 \\
\end{array}$ & meter & & RER & $\begin{array}{l}\text { techno } \\
\text { sphere }\end{array}$ \\
\hline $\begin{array}{l}\text { electricity production, photovoltaic, } 570 \mathrm{kWp} \text { open ground } \\
\text { installation, multi-Si }\end{array}$ & electricity, low voltage & $\begin{array}{r}5.62 \\
E+02 \\
\end{array}$ & $\begin{array}{l}\text { kilowatt } \\
\text { hour }\end{array}$ & & GR & $\begin{array}{l}\text { techno } \\
\text { sphere }\end{array}$ \\
\hline $\begin{array}{l}\text { electricity production, photovoltaic, } 570 \mathrm{kWp} \text { open ground } \\
\text { installation, multi-Si }\end{array}$ & electricity, low voltage & $\begin{array}{r}1.29 \\
E+02 \\
\end{array}$ & $\begin{array}{l}\text { kilowatt } \\
\text { hour }\end{array}$ & & GR & $\begin{array}{l}\text { techno } \\
\text { sphere }\end{array}$ \\
\hline end of life, carbon dioxide capture system & & $\begin{array}{l}5.00 \\
\mathrm{E}-07 \\
\end{array}$ & unit & & RoW & $\begin{array}{l}\text { techno } \\
\text { sphere }\end{array}$ \\
\hline heat production, Fresnel solar collector plant, Creta, Greece & $\begin{array}{l}\text { heat, central or small- } \\
\text { scale, Fresnel plant }\end{array}$ & $\begin{array}{r}5.40 \\
E+03 \\
\end{array}$ & $\begin{array}{l}\text { megajoul } \\
\mathrm{e}\end{array}$ & & GR & $\begin{array}{l}\text { techno } \\
\text { sphere }\end{array}$ \\
\hline market for air compressor, screw-type compressor, $300 \mathrm{~kW}$ & $\begin{array}{l}\text { air compressor, screw-type } \\
\text { compressor, } 300 \mathrm{~kW}\end{array}$ & $\begin{array}{l}4.35 \\
\mathrm{E}-06 \\
\end{array}$ & unit & & GLO & $\begin{array}{l}\text { techno } \\
\text { sphere }\end{array}$ \\
\hline market for electricity, low voltage & electricity, low voltage & $\begin{array}{r}2.42 \\
E+01 \\
\end{array}$ & $\begin{array}{l}\text { kilowatt } \\
\text { hour }\end{array}$ & & GR & $\begin{array}{l}\text { techno } \\
\text { sphere }\end{array}$ \\
\hline market for gas turbine, $10 \mathrm{MW}$ electrical & $\begin{array}{l}\text { gas turbine, } 10 \mathrm{MW} \\
\text { electrical }\end{array}$ & $\begin{array}{l}2.54 \\
\mathrm{E}-08 \\
\end{array}$ & unit & & GLO & $\begin{array}{l}\text { techno } \\
\text { sphere }\end{array}$ \\
\hline sorbent, generic & & $\begin{array}{r}3.00 \\
E+00 \\
\end{array}$ & kilogram & & RER & $\begin{array}{l}\text { techno } \\
\text { sphere }\end{array}$ \\
\hline storage unit production, Fresnel plant, Jordan, $12 \mathrm{~h}$ storage & $\begin{array}{l}\text { storage unit, Fresnel plant, } \\
\text { Jordan, } 12 \mathrm{~h} \text { storage }\end{array}$ & $\begin{array}{l}4.00 \\
\mathrm{E}-07 \\
\end{array}$ & unit & & jO & $\begin{array}{l}\text { techno } \\
\text { sphere }\end{array}$ \\
\hline transport, freight, lorry $>32$ metric ton, EURO 6 & $\begin{array}{l}\text { transport, freight, lorry } \\
>32 \text { metric ton, EURO6 }\end{array}$ & $\begin{array}{l}2.53 \\
\mathrm{E}-01 \\
\end{array}$ & $\begin{array}{l}\text { ton } \\
\text { kilomete } \\
\mathrm{r}\end{array}$ & & RoW & $\begin{array}{l}\text { techno } \\
\text { sphere }\end{array}$ \\
\hline transport, freight, sea, container ship & $\begin{array}{l}\text { transport, freight, sea, } \\
\text { container ship }\end{array}$ & $\begin{array}{r}1.16 \\
E+00 \\
\end{array}$ & $\begin{array}{l}\text { ton } \\
\text { kilomete } \\
\mathrm{r}\end{array}$ & & GLO & $\begin{array}{l}\text { techno } \\
\text { sphere }\end{array}$ \\
\hline transport, passengers, passenger aircraft, medium haul & $\begin{array}{l}\text { transport, passengers, } \\
\text { aircraft, medium haul }\end{array}$ & $\begin{array}{l}2.02 \\
\mathrm{E}-01 \\
\end{array}$ & $\begin{array}{l}\text { person } \\
\text { kilomete } \\
r\end{array}$ & & GLO & $\begin{array}{l}\text { techno } \\
\text { sphere }\end{array}$ \\
\hline $\begin{array}{l}\text { transport, pipeline, supercritical CO2, } 200 \mathrm{~km} \mathrm{w} \\
\text { recompression }\end{array}$ & & $\begin{array}{r}3.00 \\
E+02 \\
\end{array}$ & $\begin{array}{l}\text { ton } \\
\text { kilomete } \\
\mathrm{r}\end{array}$ & & RER & $\begin{array}{l}\text { techno } \\
\text { sphere }\end{array}$ \\
\hline $\begin{array}{l}\text { transport, pipeline, supercritical CO2, } 200 \mathrm{~km} \mathrm{w} / \mathrm{o} \\
\text { recompression }\end{array}$ & & $\begin{array}{r}2.00 \\
E+02 \\
\end{array}$ & $\begin{array}{l}\text { ton } \\
\text { kilomete } \\
\mathrm{r}\end{array}$ & & RER & $\begin{array}{l}\text { techno } \\
\text { sphere }\end{array}$ \\
\hline $\begin{array}{l}\text { carbon dioxide, captured from atmosphere, Autonomous } \\
\text { HTHP + PV, GR }\end{array}$ & & & ton & & GR & \\
\hline ( & Reference Product & $\begin{array}{l}\text { Amo } \\
\text { unt }\end{array}$ & Unit & Categories & $\begin{array}{l}\text { Loca } \\
\text { tion }\end{array}$ & Type \\
\hline Battery management system, $\mathrm{kWh}$ & & $\begin{array}{ll}1.84 \\
\mathrm{E}-01 \\
\end{array}$ & $\begin{array}{l}\text { kilowatt } \\
\text { hour }\end{array}$ & & GLO & $\begin{array}{l}\text { techno } \\
\text { sphere }\end{array}$ \\
\hline Carbon dioxide, fossil & & $\begin{aligned} 7.00 \\
E+00 \\
\end{aligned}$ & kilogram & $\begin{array}{l}\text { air:urban air } \\
\text { close to ground }\end{array}$ & & $\begin{array}{l}\text { biosph } \\
\text { ere }\end{array}$ \\
\hline Energy management system, $\mathrm{kWh}$ & & $\begin{array}{l}1.84 \\
\mathrm{E}-01 \\
\end{array}$ & $\begin{array}{l}\text { kilowatt } \\
\text { hour }\end{array}$ & & GLO & $\begin{array}{l}\text { techno } \\
\text { sphere }\end{array}$ \\
\hline Li-ion (NMC) & & $\begin{array}{l}1.84 \\
\mathrm{E}-01 \\
\end{array}$ & $\begin{array}{l}\text { kilowatt } \\
\text { hour }\end{array}$ & & GLO & $\begin{array}{l}\text { techno } \\
\text { sphere }\end{array}$ \\
\hline Power conditioning system, container system & & $\begin{array}{l}5.52 \\
\mathrm{E}-02 \\
\end{array}$ & kW & & GLO & $\begin{array}{l}\text { techno } \\
\text { sphere }\end{array}$ \\
\hline carbon dioxide capture system & & $\begin{array}{l}5.00 \\
\mathrm{E}-07 \\
\end{array}$ & unit & & RER & $\begin{array}{l}\text { techno } \\
\text { sphere }\end{array}$ \\
\hline drilling, deep borehole/m & & $\begin{array}{l}3.38 \\
\mathrm{E}-05\end{array}$ & meter & & RER & $\begin{array}{l}\text { techno } \\
\text { sphere }\end{array}$ \\
\hline $\begin{array}{l}\text { electricity production, photovoltaic, } 570 \mathrm{kWp} \text { open ground } \\
\text { installation, multi-Si }\end{array}$ & electricity, low voltage & $\begin{array}{r}5.62 \\
E+02 \\
\end{array}$ & $\begin{array}{l}\text { kilowatt } \\
\text { hour }\end{array}$ & & GR & $\begin{array}{l}\text { techno } \\
\text { sphere }\end{array}$ \\
\hline $\begin{array}{l}\text { electricity production, photovoltaic, } 570 \mathrm{kWp} \text { open ground } \\
\text { installation, multi-Si }\end{array}$ & electricity, low voltage & $\begin{array}{r}1.29 \\
E+02 \\
\end{array}$ & $\begin{array}{l}\text { kilowatt } \\
\text { hour }\end{array}$ & & GR & $\begin{array}{l}\text { techno } \\
\text { sphere }\end{array}$ \\
\hline
\end{tabular}




\begin{tabular}{|c|c|c|c|c|c|c|}
\hline $\begin{array}{l}\text { electricity production, photovoltaic, } 570 \mathrm{kWp} \text { open ground } \\
\text { installation, multi-Si }\end{array}$ & electricity, low voltage & $\begin{array}{r}5.81 \\
E+02 \\
\end{array}$ & $\begin{array}{l}\text { kilowatt } \\
\text { hour }\end{array}$ & & GR & $\begin{array}{l}\text { techno } \\
\text { sphere }\end{array}$ \\
\hline end of life, carbon dioxide capture system & & $\begin{array}{ll}5.00 \\
\mathrm{E}-07 \\
\end{array}$ & unit & & RoW & $\begin{array}{l}\text { techno } \\
\text { sphere }\end{array}$ \\
\hline heat pump production, brine-water, $\mathrm{CO} 2$ refrigerant, $10 \mathrm{~kW}$ & & $\begin{array}{l}8.56 \\
\mathrm{E}-04 \\
\end{array}$ & unit & & $\mathrm{CH}$ & $\begin{array}{l}\text { techno } \\
\text { sphere }\end{array}$ \\
\hline market for air compressor, screw-type compressor, 300kW & $\begin{array}{l}\text { air compressor, screw-type } \\
\text { compressor, } 300 \mathrm{~kW}\end{array}$ & $\begin{array}{l}4.35 \\
\mathrm{E}-06 \\
\end{array}$ & unit & & GLO & $\begin{array}{l}\text { techno } \\
\text { sphere }\end{array}$ \\
\hline market for electricity, low voltage & electricity, low voltage & $\begin{array}{r}2.42 \\
\mathrm{E}+01 \\
\end{array}$ & $\begin{array}{l}\text { kilowatt } \\
\text { hour }\end{array}$ & & GR & $\begin{array}{l}\text { techno } \\
\text { sphere }\end{array}$ \\
\hline market for gas turbine, $10 \mathrm{MW}$ electrical & $\begin{array}{l}\text { gas turbine, } 10 \mathrm{MW} \\
\text { electrical }\end{array}$ & $\begin{array}{ll}2.54 \\
\mathrm{E}-08 \\
\end{array}$ & unit & & GLO & $\begin{array}{l}\text { techno } \\
\text { sphere }\end{array}$ \\
\hline sorbent, generic & & $\begin{array}{r}3.00 \\
E+00 \\
\end{array}$ & kilogram & & RER & $\begin{array}{l}\text { techno } \\
\text { sphere }\end{array}$ \\
\hline transport, freight, lorry $>32$ metric ton, EURO 6 & $\begin{array}{l}\text { transport, freight, lorry } \\
>32 \text { metric ton, EURO6 }\end{array}$ & $\begin{array}{l}2.53 \\
\mathrm{E}-01 \\
\end{array}$ & $\begin{array}{l}\text { ton } \\
\text { kilomete } \\
\mathrm{r}\end{array}$ & & RoW & $\begin{array}{l}\text { techno } \\
\text { sphere }\end{array}$ \\
\hline transport, freight, sea, container ship & $\begin{array}{l}\text { transport, freight, sea, } \\
\text { container ship }\end{array}$ & $\begin{array}{r}1.16 \\
E+00\end{array}$ & $\begin{array}{l}\text { ton } \\
\text { kilomete } \\
r\end{array}$ & & GLO & $\begin{array}{l}\text { techno } \\
\text { sphere }\end{array}$ \\
\hline transport, passengers, passenger aircraft, medium haul & $\begin{array}{l}\text { transport, passengers, } \\
\text { aircraft, medium haul }\end{array}$ & $\begin{array}{l}2.02 \\
\mathrm{E}-01 \\
\end{array}$ & $\begin{array}{l}\text { person } \\
\text { kilomete } \\
r\end{array}$ & & GLO & $\begin{array}{l}\text { techno } \\
\text { sphere }\end{array}$ \\
\hline $\begin{array}{l}\text { transport, pipeline, supercritical CO2, 200km w } \\
\text { recompression }\end{array}$ & & $\begin{array}{r}3.00 \\
E+02\end{array}$ & $\begin{array}{l}\text { ton } \\
\text { kilomete } \\
\mathrm{r}\end{array}$ & & RER & $\begin{array}{l}\text { techno } \\
\text { sphere }\end{array}$ \\
\hline $\begin{array}{l}\text { transport, pipeline, supercritical CO2, 200km w/o } \\
\text { recompression }\end{array}$ & & $\begin{array}{r}2.00 \\
E+02 \\
\end{array}$ & $\begin{array}{l}\text { ton } \\
\text { kilomete } \\
r\end{array}$ & & RER & $\begin{array}{l}\text { techno } \\
\text { sphere }\end{array}$ \\
\hline $\begin{array}{l}\text { carbon dioxide, captured from atmosphere, Waste heat + } \\
\text { Grid, JO }\end{array}$ & & & ton & & jO & \\
\hline Name & Reference Product & $\begin{array}{l}\text { Amo } \\
\text { unt }\end{array}$ & Unit & Categories & $\begin{array}{l}\text { Loca } \\
\text { tion }\end{array}$ & Type \\
\hline Carbon dioxide, fossil & & $\begin{array}{r}1.40 \\
\mathrm{E}+00 \\
\end{array}$ & kilogram & $\begin{array}{l}\text { air:urban air } \\
\text { close to ground }\end{array}$ & & $\begin{array}{l}\text { biosph } \\
\text { ere }\end{array}$ \\
\hline carbon dioxide capture system & & $\begin{array}{l}5.00 \\
\mathrm{E}-07 \\
\end{array}$ & unit & & RER & $\begin{array}{l}\text { techno } \\
\text { sphere }\end{array}$ \\
\hline drilling, deep borehole/m & & $\begin{array}{l}3.38 \\
\mathrm{E}-05 \\
\end{array}$ & meter & & RER & $\begin{array}{l}\text { techno } \\
\text { sphere }\end{array}$ \\
\hline end of life, carbon dioxide capture system & & $\begin{array}{l}5.00 \\
\mathrm{E}-07 \\
\end{array}$ & unit & & RoW & $\begin{array}{l}\text { techno } \\
\text { sphere }\end{array}$ \\
\hline $\begin{array}{l}\text { heat, from municipal waste incineration to generic market for } \\
\text { heat district or industrial, other than natural gas }\end{array}$ & $\begin{array}{l}\text { heat, district or industrial, } \\
\text { other than natural gas }\end{array}$ & $\begin{array}{r}5.40 \\
E+03 \\
\end{array}$ & $\begin{array}{l}\text { megajoul } \\
\mathrm{e}\end{array}$ & & RoW & $\begin{array}{l}\text { techno } \\
\text { sphere }\end{array}$ \\
\hline market for air compressor, screw-type compressor, $300 \mathrm{~kW}$ & $\begin{array}{l}\text { air compressor, screw-type } \\
\text { compressor, } 300 \mathrm{~kW}\end{array}$ & $\begin{array}{l}4.35 \\
\mathrm{E}-06 \\
\end{array}$ & unit & & GLO & $\begin{array}{l}\text { techno } \\
\text { sphere }\end{array}$ \\
\hline market for electricity, low voltage & electricity, low voltage & $\begin{aligned} 5.00 \\
E+02 \\
\end{aligned}$ & $\begin{array}{l}\text { kilowatt } \\
\text { hour }\end{array}$ & & JO & $\begin{array}{l}\text { techno } \\
\text { sphere }\end{array}$ \\
\hline market for electricity, low voltage & electricity, low voltage & $\begin{aligned} 1.14 \\
E+02 \\
\end{aligned}$ & $\begin{array}{l}\text { kilowatt } \\
\text { hour }\end{array}$ & & JO & $\begin{array}{l}\text { techno } \\
\text { sphere }\end{array}$ \\
\hline market for electricity, low voltage & electricity, low voltage & $\begin{array}{r}2.42 \\
E+01 \\
\end{array}$ & $\begin{array}{l}\text { kilowatt } \\
\text { hour }\end{array}$ & & JO & $\begin{array}{l}\text { techno } \\
\text { sphere }\end{array}$ \\
\hline market for gas turbine, $10 \mathrm{MW}$ electrical & $\begin{array}{l}\text { gas turbine, 10MW } \\
\text { electrical }\end{array}$ & $\begin{array}{l}2.54 \\
\mathrm{E}-08 \\
\end{array}$ & unit & & GLO & $\begin{array}{l}\text { techno } \\
\text { sphere }\end{array}$ \\
\hline sorbent, generic & & $\begin{array}{r}3.00 \\
E+00 \\
\end{array}$ & kilogram & & RER & $\begin{array}{l}\text { techno } \\
\text { sphere }\end{array}$ \\
\hline transport, freight, lorry $>32$ metric ton, EURO4 & $\begin{array}{l}\text { transport, freight, lorry } \\
>32 \text { metric ton, EURO4 }\end{array}$ & $\begin{array}{l}9.44 \\
\mathrm{E}-02 \\
\end{array}$ & $\begin{array}{l}\text { ton } \\
\text { kilomete } \\
\mathrm{r} \\
\end{array}$ & & RoW & $\begin{array}{l}\text { techno } \\
\text { sphere }\end{array}$ \\
\hline transport, freight, lorry $>32$ metric ton, EURO 6 & $\begin{array}{l}\text { transport, freight, lorry } \\
>32 \text { metric ton, EURO6 }\end{array}$ & $\begin{array}{l}2.53 \\
\mathrm{E}-01 \\
\end{array}$ & $\begin{array}{l}\text { ton } \\
\text { kilomete } \\
\mathrm{r} \\
\end{array}$ & & RoW & $\begin{array}{l}\text { techno } \\
\text { sphere }\end{array}$ \\
\hline transport, freight, sea, container ship & $\begin{array}{l}\text { transport, freight, sea, } \\
\text { container ship }\end{array}$ & $\begin{array}{r}1.71 \\
\mathrm{E}+00 \\
\end{array}$ & $\begin{array}{l}\text { ton } \\
\text { kilomete } \\
\mathrm{r}\end{array}$ & & GLO & $\begin{array}{l}\text { techno } \\
\text { sphere }\end{array}$ \\
\hline transport, passengers, passenger aircraft, medium haul & $\begin{array}{l}\text { transport, passengers, } \\
\text { aircraft, medium haul }\end{array}$ & $\begin{array}{l}3.00 \\
\mathrm{E}-01 \\
\end{array}$ & $\begin{array}{l}\text { person } \\
\text { kilomete } \\
r\end{array}$ & & GLO & $\begin{array}{l}\text { techno } \\
\text { sphere }\end{array}$ \\
\hline $\begin{array}{l}\text { transport, pipeline, supercritical CO2, } 200 \mathrm{~km} \text { w/o } \\
\text { recompression }\end{array}$ & & $\begin{array}{r}1.00 \\
E+02 \\
\end{array}$ & $\begin{array}{l}\text { ton } \\
\text { kilomete } \\
\mathrm{r}\end{array}$ & & RER & $\begin{array}{l}\text { techno } \\
\text { sphere }\end{array}$ \\
\hline $\begin{array}{l}\text { carbon dioxide, captured from atmosphere, Waste heat + } \\
\text { PV + Battery, JO }\end{array}$ & & & ton & & JO & \\
\hline Name & Reference Product & $\begin{array}{l}\text { Amo } \\
\text { unt }\end{array}$ & Unit & Categories & $\begin{array}{l}\text { Loca } \\
\text { tion }\end{array}$ & Type \\
\hline Battery management system, kWh & & $\begin{array}{l}9.99 \\
\mathrm{E}-02 \\
\end{array}$ & $\begin{array}{l}\text { kilowatt } \\
\text { hour }\end{array}$ & & GLO & $\begin{array}{l}\text { techno } \\
\text { sphere }\end{array}$ \\
\hline
\end{tabular}




\begin{tabular}{|c|c|c|c|c|c|c|}
\hline Carbon dioxide, fossil & & $\begin{array}{r}1.40 \\
\mathrm{E}+00\end{array}$ & kilogram & $\begin{array}{l}\text { air:urban air } \\
\text { close to ground }\end{array}$ & & $\begin{array}{l}\text { biosph } \\
\text { ere }\end{array}$ \\
\hline Energy management system, kWh & & $\begin{array}{l}9.99 \\
\mathrm{E}-02 \\
\end{array}$ & $\begin{array}{l}\text { kilowatt } \\
\text { hour }\end{array}$ & & GLO & $\begin{array}{l}\text { techno } \\
\text { sphere }\end{array}$ \\
\hline Li-ion (NMC) & & $\begin{array}{l}9.99 \\
\mathrm{E}-02\end{array}$ & $\begin{array}{l}\text { kilowatt } \\
\text { hour }\end{array}$ & & GLO & $\begin{array}{l}\text { techno } \\
\text { sphere }\end{array}$ \\
\hline Power conditioning system, container system & & $\begin{array}{l}3.00 \\
\mathrm{E}-02\end{array}$ & kW & & GLO & $\begin{array}{l}\text { techno } \\
\text { sphere }\end{array}$ \\
\hline carbon dioxide capture system & & $\begin{array}{l}5.00 \\
E-07\end{array}$ & unit & & RER & $\begin{array}{l}\text { techno } \\
\text { sphere }\end{array}$ \\
\hline drilling, deep borehole/m & & $\begin{array}{l}3.38 \\
\mathrm{E}-05 \\
\end{array}$ & meter & & RER & $\begin{array}{l}\text { techno } \\
\text { sphere }\end{array}$ \\
\hline $\begin{array}{l}\text { electricity production, photovoltaic, } 570 \mathrm{kWp} \text { open ground } \\
\text { installation, multi-Si }\end{array}$ & electricity, low voltage & $\begin{array}{r}5.62 \\
\mathrm{E}+02 \\
\end{array}$ & $\begin{array}{l}\text { kilowatt } \\
\text { hour }\end{array}$ & & JO & $\begin{array}{l}\text { techno } \\
\text { sphere }\end{array}$ \\
\hline $\begin{array}{l}\text { electricity production, photovoltaic, } 570 \mathrm{kWp} \text { open ground } \\
\text { installation, multi-Si }\end{array}$ & electricity, low voltage & $\begin{array}{r}1.29 \\
\mathrm{E}+02 \\
\end{array}$ & $\begin{array}{l}\text { kilowatt } \\
\text { hour }\end{array}$ & & JO & $\begin{array}{l}\text { techno } \\
\text { sphere }\end{array}$ \\
\hline end of life, carbon dioxide capture system & & $\begin{array}{l}5.00 \\
\mathrm{E}-07 \\
\end{array}$ & unit & & RoW & $\begin{array}{l}\text { techno } \\
\text { sphere }\end{array}$ \\
\hline $\begin{array}{l}\text { heat, from municipal waste incineration to generic market for } \\
\text { heat district or industrial, other than natural gas }\end{array}$ & $\begin{array}{l}\text { heat, district or industrial, } \\
\text { other than natural gas }\end{array}$ & $\begin{array}{r}5.40 \\
E+03 \\
\end{array}$ & $\begin{array}{l}\text { megajoul } \\
\mathrm{e}\end{array}$ & & RoW & $\begin{array}{l}\text { techno } \\
\text { sphere }\end{array}$ \\
\hline market for air compressor, screw-type compressor, 300kW & $\begin{array}{l}\text { air compressor, screw-type } \\
\text { compressor, } 300 \mathrm{~kW}\end{array}$ & $\begin{array}{l}4.35 \\
\mathrm{E}-06 \\
\end{array}$ & unit & & GLO & $\begin{array}{l}\text { techno } \\
\text { sphere }\end{array}$ \\
\hline market for electricity, low voltage & electricity, low voltage & $\begin{array}{r}2.42 \\
E+01\end{array}$ & $\begin{array}{l}\text { kilowatt } \\
\text { hour }\end{array}$ & & JO & $\begin{array}{l}\text { techno } \\
\text { sphere }\end{array}$ \\
\hline market for gas turbine, $10 \mathrm{MW}$ electrical & $\begin{array}{l}\text { gas turbine, } 10 \mathrm{MW} \\
\text { electrical }\end{array}$ & $\begin{array}{l}2.54 \\
\mathrm{E}-08 \\
\end{array}$ & unit & & GLO & $\begin{array}{l}\text { techno } \\
\text { sphere }\end{array}$ \\
\hline sorbent, generic & & $\begin{array}{r}3.00 \\
E+00\end{array}$ & kilogram & & RER & $\begin{array}{l}\text { techno } \\
\text { sphere }\end{array}$ \\
\hline transport, freight, lorry >32 metric ton, EURO4 & $\begin{array}{l}\text { transport, freight, lorry } \\
>32 \text { metric ton, EURO4 }\end{array}$ & $\begin{array}{l}9.44 \\
\mathrm{E}-02\end{array}$ & $\begin{array}{l}\text { ton } \\
\text { kilomete } \\
\mathrm{r}\end{array}$ & & RoW & $\begin{array}{l}\text { techno } \\
\text { sphere }\end{array}$ \\
\hline transport, freight, lorry $>32$ metric ton, EURO 6 & $\begin{array}{l}\text { transport, freight, lorry } \\
>32 \text { metric ton, EURO6 }\end{array}$ & $\begin{array}{l}2.53 \\
\mathrm{E}-01 \\
\end{array}$ & $\begin{array}{l}\text { ton } \\
\text { kilomete } \\
\mathrm{r}\end{array}$ & & RoW & $\begin{array}{l}\text { techno } \\
\text { sphere }\end{array}$ \\
\hline transport, freight, sea, container ship & $\begin{array}{l}\text { transport, freight, sea, } \\
\text { container ship }\end{array}$ & $\begin{array}{r}1.71 \\
\mathrm{E}+00 \\
\end{array}$ & $\begin{array}{l}\text { ton } \\
\text { kilomete } \\
\mathrm{r}\end{array}$ & & GLO & $\begin{array}{l}\text { techno } \\
\text { sphere }\end{array}$ \\
\hline transport, passengers, passenger aircraft, medium haul & $\begin{array}{l}\text { transport, passengers, } \\
\text { aircraft, medium haul }\end{array}$ & $\begin{array}{l}3.00 \\
\mathrm{E}-01 \\
\end{array}$ & $\begin{array}{l}\text { person } \\
\text { kilomete } \\
\mathrm{r}\end{array}$ & & GLO & $\begin{array}{l}\text { techno } \\
\text { sphere }\end{array}$ \\
\hline $\begin{array}{l}\text { transport, pipeline, supercritical CO2, } 200 \mathrm{~km} \text { w/o } \\
\text { recompression }\end{array}$ & & $\begin{array}{r}1.00 \\
E+02 \\
\end{array}$ & $\begin{array}{l}\text { ton } \\
\text { kilomete } \\
r\end{array}$ & & RER & $\begin{array}{l}\text { techno } \\
\text { sphere }\end{array}$ \\
\hline carbon dioxide, captured from atmosphere, HTHP + Grid, JO & & & ton & & $\mathrm{JO}$ & \\
\hline Name & Reference Product & $\begin{array}{l}\text { Amo } \\
\text { unt }\end{array}$ & Unit & Categories & $\begin{array}{l}\text { Loca } \\
\text { tion }\end{array}$ & Type \\
\hline Carbon dioxide, fossil & & $\begin{array}{r}1.40 \\
\mathrm{E}+00 \\
\end{array}$ & kilogram & $\begin{array}{l}\text { air:urban air } \\
\text { close to ground }\end{array}$ & & $\begin{array}{l}\text { biosph } \\
\text { ere }\end{array}$ \\
\hline carbon dioxide capture system & & $\begin{array}{l}5.00 \\
\mathrm{E}-07 \\
\end{array}$ & unit & & RER & $\begin{array}{l}\text { techno } \\
\text { sphere }\end{array}$ \\
\hline drilling, deep borehole/m & & $\begin{array}{l}3.38 \\
\mathrm{E}-05 \\
\end{array}$ & meter & & RER & $\begin{array}{l}\text { techno } \\
\text { sphere }\end{array}$ \\
\hline end of life, carbon dioxide capture system & & $\begin{array}{l}5.00 \\
\mathrm{E}-07\end{array}$ & unit & & RoW & $\begin{array}{l}\text { techno } \\
\text { sphere }\end{array}$ \\
\hline heat pump production, brine-water, $\mathrm{CO} 2$ refrigerant, $10 \mathrm{~kW}$ & & $\begin{array}{l}8.56 \\
\mathrm{E}-04 \\
\end{array}$ & unit & & $\mathrm{CH}$ & $\begin{array}{l}\text { techno } \\
\text { sphere }\end{array}$ \\
\hline market for air compressor, screw-type compressor, 300kW & $\begin{array}{l}\text { air compressor, screw-type } \\
\text { compressor, } 300 \mathrm{~kW}\end{array}$ & $\begin{array}{l}4.35 \\
\mathrm{E}-06 \\
\end{array}$ & unit & & GLO & $\begin{array}{l}\text { techno } \\
\text { sphere }\end{array}$ \\
\hline market for electricity, low voltage & electricity, low voltage & $\begin{array}{r}5.00 \\
E+02 \\
\end{array}$ & $\begin{array}{l}\text { kilowatt } \\
\text { hour }\end{array}$ & & JO & $\begin{array}{l}\text { techno } \\
\text { sphere }\end{array}$ \\
\hline market for electricity, low voltage & electricity, low voltage & $\begin{array}{r}1.14 \\
\mathrm{E}+02 \\
\end{array}$ & $\begin{array}{l}\text { kilowatt } \\
\text { hour }\end{array}$ & & jO & $\begin{array}{l}\text { techno } \\
\text { sphere }\end{array}$ \\
\hline market for electricity, low voltage & electricity, low voltage & $\begin{array}{r}5.17 \\
\mathrm{E}+02 \\
\end{array}$ & $\begin{array}{l}\text { kilowatt } \\
\text { hour }\end{array}$ & & JO & $\begin{array}{l}\text { techno } \\
\text { sphere }\end{array}$ \\
\hline market for electricity, low voltage & electricity, low voltage & $\begin{array}{r}2.42 \\
E+01 \\
\end{array}$ & $\begin{array}{l}\text { kilowatt } \\
\text { hour }\end{array}$ & & JO & $\begin{array}{l}\text { techno } \\
\text { sphere }\end{array}$ \\
\hline market for gas turbine, $10 \mathrm{MW}$ electrical & $\begin{array}{l}\text { gas turbine, 10MW } \\
\text { electrical }\end{array}$ & $\begin{array}{l}2.54 \\
\mathrm{E}-08 \\
\end{array}$ & unit & & GLO & $\begin{array}{l}\text { techno } \\
\text { sphere }\end{array}$ \\
\hline sorbent, generic & & $\begin{array}{r}3.00 \\
E+00 \\
\end{array}$ & kilogram & & RER & $\begin{array}{l}\text { techno } \\
\text { sphere }\end{array}$ \\
\hline transport, freight, lorry $>32$ metric ton, EURO 4 & $\begin{array}{l}\text { transport, freight, lorry } \\
>32 \text { metric ton, EURO4 }\end{array}$ & $\begin{array}{l}9.44 \\
\mathrm{E}-02 \\
\end{array}$ & $\begin{array}{l}\text { ton } \\
\text { kilomete } \\
\mathrm{r}\end{array}$ & & RoW & $\begin{array}{l}\text { techno } \\
\text { sphere } \\
\end{array}$ \\
\hline transport, freight, lorry $>32$ metric ton, EURO 6 & $\begin{array}{l}\text { transport, freight, lorry } \\
>32 \text { metric ton, EURO6 }\end{array}$ & $\begin{array}{l}2.53 \\
\mathrm{E}-01 \\
\end{array}$ & $\begin{array}{l}\text { ton } \\
\text { kilomete } \\
r\end{array}$ & & RoW & $\begin{array}{l}\text { techno } \\
\text { sphere }\end{array}$ \\
\hline
\end{tabular}




\begin{tabular}{|c|c|c|c|c|c|c|}
\hline transport, freight, sea, container ship & $\begin{array}{l}\text { transport, freight, sea, } \\
\text { container ship }\end{array}$ & $\begin{array}{r}1.71 \\
E+00\end{array}$ & $\begin{array}{l}\text { ton } \\
\text { kilomete } \\
r\end{array}$ & & GLO & $\begin{array}{l}\text { techno } \\
\text { sphere }\end{array}$ \\
\hline transport, passengers, passenger aircraft, medium haul & $\begin{array}{l}\text { transport, passengers, } \\
\text { aircraft, medium haul }\end{array}$ & $\begin{array}{l}3.00 \\
\mathrm{E}-01 \\
\end{array}$ & $\begin{array}{l}\text { person } \\
\text { kilomete } \\
\mathrm{r}\end{array}$ & & GLO & $\begin{array}{l}\text { techno } \\
\text { sphere }\end{array}$ \\
\hline $\begin{array}{l}\text { transport, pipeline, supercritical CO2, } 200 \mathrm{~km} \mathrm{w} / \mathrm{o} \\
\text { recompression }\end{array}$ & & $\begin{array}{r}1.00 \\
E+02\end{array}$ & $\begin{array}{l}\text { ton } \\
\text { kilomete } \\
r\end{array}$ & & RER & $\begin{array}{l}\text { techno } \\
\text { sphere }\end{array}$ \\
\hline $\begin{array}{l}\text { carbon dioxide, captured from atmosphere, Autonomous } \\
\text { Fresnel + PV, JO }\end{array}$ & & & ton & & JO & \\
\hline Name & Reference Product & $\begin{array}{l}\text { Amo } \\
\text { unt }\end{array}$ & Unit & Categories & $\begin{array}{l}\text { Loca } \\
\text { tion }\end{array}$ & Type \\
\hline Battery management system, kWh & & $\begin{array}{ll}1.04 \\
\mathrm{E}-01\end{array}$ & $\begin{array}{l}\text { kilowatt } \\
\text { hour }\end{array}$ & & GLO & $\begin{array}{l}\text { techno } \\
\text { sphere }\end{array}$ \\
\hline Carbon dioxide, fossil & & $\begin{array}{r}1.40 \\
E+00\end{array}$ & kilogram & $\begin{array}{l}\text { air:urban air } \\
\text { close to ground }\end{array}$ & & $\begin{array}{l}\text { biosph } \\
\text { ere }\end{array}$ \\
\hline Energy management system, $\mathrm{kWh}$ & & $\begin{array}{ll}1.04 \\
\mathrm{E}-01 \\
\end{array}$ & $\begin{array}{l}\text { kilowatt } \\
\text { hour }\end{array}$ & & GLO & $\begin{array}{l}\text { techno } \\
\text { sphere }\end{array}$ \\
\hline Li-ion (NMC) & & $\begin{array}{l}1.04 \\
E-01 \\
\end{array}$ & $\begin{array}{l}\text { kilowatt } \\
\text { hour }\end{array}$ & & GLO & $\begin{array}{l}\text { techno } \\
\text { sphere }\end{array}$ \\
\hline Power conditioning system, container system & & $\begin{array}{ll}3.13 \\
\mathrm{E}-02\end{array}$ & $\mathrm{~kW}$ & & GLO & $\begin{array}{l}\text { techno } \\
\text { sphere }\end{array}$ \\
\hline carbon dioxide capture system & & $\begin{array}{l}5.00 \\
\mathrm{E}-07 \\
\end{array}$ & unit & & RER & $\begin{array}{l}\text { techno } \\
\text { sphere }\end{array}$ \\
\hline drilling, deep borehole/m & & $\begin{array}{ll}3.38 \\
\mathrm{E}-05\end{array}$ & meter & & RER & $\begin{array}{l}\text { techno } \\
\text { sphere }\end{array}$ \\
\hline $\begin{array}{l}\text { electricity production, photovoltaic, } 570 \mathrm{kWp} \text { open ground } \\
\text { installation, multi-Si }\end{array}$ & electricity, low voltage & $\begin{array}{r}5.62 \\
E+02 \\
\end{array}$ & $\begin{array}{l}\text { kilowatt } \\
\text { hour }\end{array}$ & & jO & $\begin{array}{l}\text { techno } \\
\text { sphere }\end{array}$ \\
\hline $\begin{array}{l}\text { electricity production, photovoltaic, } 570 \mathrm{kWp} \text { open ground } \\
\text { installation, multi-Si }\end{array}$ & electricity, low voltage & $\begin{array}{r}1.29 \\
E+02 \\
\end{array}$ & $\begin{array}{l}\text { kilowatt } \\
\text { hour }\end{array}$ & & jo & $\begin{array}{l}\text { techno } \\
\text { sphere }\end{array}$ \\
\hline end of life, carbon dioxide capture system & & $\begin{array}{ll}5.00 \\
\mathrm{E}-07 \\
\end{array}$ & unit & & RoW & $\begin{array}{l}\text { techno } \\
\text { sphere }\end{array}$ \\
\hline heat production, Fresnel solar collector plant, Amman, Jordan & $\begin{array}{l}\text { heat, central or small- } \\
\text { scale, Fresnel plant }\end{array}$ & $\begin{array}{r}5.40 \\
E+03 \\
\end{array}$ & $\begin{array}{l}\text { megajoul } \\
\mathrm{e}\end{array}$ & & JO & $\begin{array}{l}\text { techno } \\
\text { sphere }\end{array}$ \\
\hline market for air compressor, screw-type compressor, $300 \mathrm{~kW}$ & $\begin{array}{l}\text { air compressor, screw-type } \\
\text { compressor, } 300 \mathrm{~kW}\end{array}$ & $\begin{array}{l}4.35 \\
E-06\end{array}$ & unit & & GLO & $\begin{array}{l}\text { techno } \\
\text { sphere }\end{array}$ \\
\hline market for electricity, low voltage & electricity, low voltage & $\begin{array}{r}2.42 \\
E+01 \\
\end{array}$ & $\begin{array}{l}\text { kilowatt } \\
\text { hour }\end{array}$ & & jO & $\begin{array}{l}\text { techno } \\
\text { sphere }\end{array}$ \\
\hline market for gas turbine, $10 \mathrm{MW}$ electrical & $\begin{array}{l}\text { gas turbine, } 10 \mathrm{MW} \\
\text { electrical }\end{array}$ & $\begin{array}{l}2.54 \\
\mathrm{E}-08 \\
\end{array}$ & unit & & GLO & $\begin{array}{l}\text { techno } \\
\text { sphere }\end{array}$ \\
\hline sorbent, generic & & $\begin{array}{r}3.00 \\
E+00 \\
\end{array}$ & kilogram & & RER & $\begin{array}{l}\text { techno } \\
\text { sphere }\end{array}$ \\
\hline storage unit production, Fresnel plant, Jordan, $12 \mathrm{~h}$ storage & $\begin{array}{l}\text { storage unit, Fresnel plant, } \\
\text { Jordan, } 12 \mathrm{~h} \text { storage }\end{array}$ & $\begin{array}{l}4.00 \\
\mathrm{E}-07 \\
\end{array}$ & unit & & jO & $\begin{array}{l}\text { techno } \\
\text { sphere }\end{array}$ \\
\hline transport, freight, lorry $>32$ metric ton, EURO 4 & $\begin{array}{l}\text { transport, freight, lorry } \\
>32 \text { metric ton, EURO4 }\end{array}$ & $\begin{array}{l}9.44 \\
\mathrm{E}-02\end{array}$ & $\begin{array}{l}\text { ton } \\
\text { kilomete } \\
r\end{array}$ & & RoW & $\begin{array}{l}\text { techno } \\
\text { sphere }\end{array}$ \\
\hline transport, freight, lorry $>32$ metric ton, EURO 6 & $\begin{array}{l}\text { transport, freight, lorry } \\
>32 \text { metric ton, EURO6 }\end{array}$ & $\begin{array}{l}2.53 \\
\mathrm{E}-01 \\
\end{array}$ & $\begin{array}{l}\text { ton } \\
\text { kilomete } \\
\mathrm{r}\end{array}$ & & RoW & $\begin{array}{l}\text { techno } \\
\text { sphere }\end{array}$ \\
\hline transport, freight, sea, container ship & $\begin{array}{l}\text { transport, freight, sea, } \\
\text { container ship }\end{array}$ & $\begin{array}{r}1.71 \\
E+00\end{array}$ & $\begin{array}{l}\text { ton } \\
\text { kilomete } \\
r\end{array}$ & & GLO & $\begin{array}{l}\text { techno } \\
\text { sphere }\end{array}$ \\
\hline transport, passengers, passenger aircraft, medium haul & $\begin{array}{l}\text { transport, passengers, } \\
\text { aircraft, medium haul }\end{array}$ & $\begin{array}{l}3.00 \\
\mathrm{E}-01 \\
\end{array}$ & $\begin{array}{l}\text { person } \\
\text { kilomete } \\
r\end{array}$ & & GLO & $\begin{array}{l}\text { techno } \\
\text { sphere }\end{array}$ \\
\hline $\begin{array}{l}\text { transport, pipeline, supercritical CO2, 200km w/o } \\
\text { recompression }\end{array}$ & & $\begin{array}{r}1.00 \\
E+02 \\
\end{array}$ & $\begin{array}{l}\text { ton } \\
\text { kilomete } \\
\mathrm{r}\end{array}$ & & RER & $\begin{array}{l}\text { techno } \\
\text { sphere }\end{array}$ \\
\hline $\begin{array}{l}\text { carbon dioxide, captured from atmosphere, Autonomous } \\
\text { HTHP + PV, JO }\end{array}$ & & & ton & & JO & \\
\hline Name & Reference Product & $\begin{array}{l}\text { Amo } \\
\text { unt }\end{array}$ & Unit & Categories & $\begin{array}{l}\text { Loca } \\
\text { tion }\end{array}$ & Type \\
\hline Battery management system, kWh & & $\begin{array}{l}1.84 \\
\mathrm{E}-01 \\
\end{array}$ & $\begin{array}{l}\text { kilowatt } \\
\text { hour }\end{array}$ & & GLO & $\begin{array}{l}\text { techno } \\
\text { sphere }\end{array}$ \\
\hline Carbon dioxide, fossil & & $\begin{array}{r}1.40 \\
E+00 \\
\end{array}$ & kilogram & $\begin{array}{l}\text { air:urban air } \\
\text { close to ground }\end{array}$ & & $\begin{array}{l}\text { biosph } \\
\text { ere }\end{array}$ \\
\hline Energy management system, $\mathrm{kWh}$ & & $\begin{array}{l}1.84 \\
\mathrm{E}-01 \\
\end{array}$ & $\begin{array}{l}\text { kilowatt } \\
\text { hour }\end{array}$ & & GLO & $\begin{array}{l}\text { techno } \\
\text { sphere }\end{array}$ \\
\hline Li-ion (NMC) & & $\begin{array}{l}1.84 \\
\mathrm{E}-01 \\
\end{array}$ & $\begin{array}{l}\text { kilowatt } \\
\text { hour }\end{array}$ & & GLO & $\begin{array}{l}\text { techno } \\
\text { sphere }\end{array}$ \\
\hline Power conditioning system, container system & & $\begin{array}{l}5.52 \\
\mathrm{E}-02 \\
\end{array}$ & kW & & GLO & $\begin{array}{l}\text { techno } \\
\text { sphere }\end{array}$ \\
\hline carbon dioxide capture system & & $\begin{array}{l}5.00 \\
E-07 \\
\end{array}$ & unit & & RER & $\begin{array}{l}\text { techno } \\
\text { sphere }\end{array}$ \\
\hline
\end{tabular}




\begin{tabular}{|c|c|c|c|c|c|c|}
\hline drilling, deep borehole/m & & $\begin{array}{l}3.38 \\
\mathrm{E}-05 \\
\end{array}$ & meter & & RER & $\begin{array}{l}\text { techno } \\
\text { sphere }\end{array}$ \\
\hline $\begin{array}{l}\text { electricity production, photovoltaic, } 570 \mathrm{kWp} \text { open ground } \\
\text { installation, multi-Si }\end{array}$ & electricity, low voltage & $\begin{array}{r}5.62 \\
\mathrm{E}+02 \\
\end{array}$ & $\begin{array}{l}\text { kilowatt } \\
\text { hour }\end{array}$ & & JO & $\begin{array}{l}\text { techno } \\
\text { sphere }\end{array}$ \\
\hline $\begin{array}{l}\text { electricity production, photovoltaic, } 570 \mathrm{kWp} \text { open ground } \\
\text { installation, multi-Si }\end{array}$ & electricity, low voltage & $\begin{array}{r}1.29 \\
\mathrm{E}+02 \\
\end{array}$ & $\begin{array}{l}\text { kilowatt } \\
\text { hour }\end{array}$ & & JO & $\begin{array}{l}\text { techno } \\
\text { sphere }\end{array}$ \\
\hline $\begin{array}{l}\text { electricity production, photovoltaic, 570kWp open ground } \\
\text { installation, multi-Si }\end{array}$ & electricity, low voltage & $\begin{aligned} 5.81 \\
\mathrm{E}+02 \\
\end{aligned}$ & $\begin{array}{l}\text { kilowatt } \\
\text { hour }\end{array}$ & & JO & $\begin{array}{l}\text { techno } \\
\text { sphere }\end{array}$ \\
\hline end of life, carbon dioxide capture system & & $\begin{array}{l}5.00 \\
\mathrm{E}-07 \\
\end{array}$ & unit & & RoW & $\begin{array}{l}\text { techno } \\
\text { sphere }\end{array}$ \\
\hline heat pump production, brine-water, $\mathrm{CO} 2$ refrigerant, $10 \mathrm{~kW}$ & & $\begin{array}{l}8.56 \\
E-04\end{array}$ & unit & & $\mathrm{CH}$ & $\begin{array}{l}\text { techno } \\
\text { sphere }\end{array}$ \\
\hline market for air compressor, screw-type compressor, $300 \mathrm{~kW}$ & $\begin{array}{l}\text { air compressor, screw-type } \\
\text { compressor, } 300 \mathrm{~kW}\end{array}$ & $\begin{array}{l}4.35 \\
\mathrm{E}-06\end{array}$ & unit & & GLO & $\begin{array}{l}\text { techno } \\
\text { sphere }\end{array}$ \\
\hline market for electricity, low voltage & electricity, low voltage & $\begin{array}{r}2.42 \\
\mathrm{E}+01 \\
\end{array}$ & $\begin{array}{l}\text { kilowatt } \\
\text { hour }\end{array}$ & & $\mathrm{JO}$ & $\begin{array}{l}\text { techno } \\
\text { sphere }\end{array}$ \\
\hline market for gas turbine, $10 \mathrm{MW}$ electrical & $\begin{array}{l}\text { gas turbine, } 10 \mathrm{MW} \\
\text { electrical }\end{array}$ & $\begin{array}{l}2.54 \\
\mathrm{E}-08 \\
\end{array}$ & unit & & GLO & $\begin{array}{l}\text { techno } \\
\text { sphere }\end{array}$ \\
\hline sorbent, generic & & $\begin{array}{r}3.00 \\
\mathrm{E}+00 \\
\end{array}$ & kilogram & & RER & $\begin{array}{l}\text { techno } \\
\text { sphere }\end{array}$ \\
\hline transport, freight, lorry $>32$ metric ton, EURO4 & $\begin{array}{l}\text { transport, freight, lorry } \\
>32 \text { metric ton, EURO4 }\end{array}$ & $\begin{array}{l}9.44 \\
\mathrm{E}-02 \\
\end{array}$ & $\begin{array}{l}\text { ton } \\
\text { kilomete } \\
\mathrm{r}\end{array}$ & & RoW & $\begin{array}{l}\text { techno } \\
\text { sphere }\end{array}$ \\
\hline transport, freight, lorry $>32$ metric ton, EURO 6 & $\begin{array}{l}\text { transport, freight, lorry } \\
>32 \text { metric ton, EURO6 }\end{array}$ & $\begin{array}{l}2.53 \\
\mathrm{E}-01 \\
\end{array}$ & $\begin{array}{l}\text { ton } \\
\text { kilomete } \\
\mathrm{r}\end{array}$ & & RoW & $\begin{array}{l}\text { techno } \\
\text { sphere }\end{array}$ \\
\hline transport, freight, sea, container ship & $\begin{array}{l}\text { transport, freight, sea, } \\
\text { container ship }\end{array}$ & $\begin{array}{r}1.71 \\
E+00\end{array}$ & $\begin{array}{l}\text { ton } \\
\text { kilomete } \\
r\end{array}$ & & GLO & $\begin{array}{l}\text { techno } \\
\text { sphere }\end{array}$ \\
\hline transport, passengers, passenger aircraft, medium haul & $\begin{array}{l}\text { transport, passengers, } \\
\text { aircraft, medium haul }\end{array}$ & $\begin{array}{l}3.00 \\
\mathrm{E}-01 \\
\end{array}$ & $\begin{array}{l}\text { person } \\
\text { kilomete } \\
r\end{array}$ & & GLO & $\begin{array}{l}\text { techno } \\
\text { sphere }\end{array}$ \\
\hline $\begin{array}{l}\text { transport, pipeline, supercritical } \mathrm{CO} 2,200 \mathrm{~km} \text { w/o } \\
\text { recompression }\end{array}$ & & $\begin{array}{r}1.00 \\
\mathrm{E}+02 \\
\end{array}$ & $\begin{array}{l}\text { ton } \\
\text { kilomete } \\
\mathrm{r}\end{array}$ & & RER & $\begin{array}{l}\text { techno } \\
\text { sphere }\end{array}$ \\
\hline $\begin{array}{l}\text { carbon dioxide, captured from atmosphere, Waste heat + } \\
\text { Grid, } \mathrm{MX}\end{array}$ & & & ton & & $\mathrm{MX}$ & \\
\hline 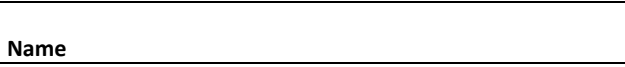 & Reference Product & $\begin{array}{l}\text { Amo } \\
\text { unt }\end{array}$ & Unit & Categories & $\begin{array}{l}\text { Loca } \\
\text { tion }\end{array}$ & Type \\
\hline Carbon dioxide, fossil & & $\begin{array}{r}2.10 \\
\mathrm{E}+01 \\
\end{array}$ & kilogram & $\begin{array}{l}\text { air:urban air } \\
\text { close to ground }\end{array}$ & & $\begin{array}{l}\text { biosph } \\
\text { ere }\end{array}$ \\
\hline carbon dioxide capture system & & $\begin{array}{l}5.00 \\
\mathrm{E}-07\end{array}$ & unit & & RER & $\begin{array}{l}\text { techno } \\
\text { sphere }\end{array}$ \\
\hline drilling, deep borehole/m & & $\begin{array}{l}3.38 \\
\mathrm{E}-05 \\
\end{array}$ & meter & & RER & $\begin{array}{l}\text { techno } \\
\text { sphere }\end{array}$ \\
\hline end of life, carbon dioxide capture system & & $\begin{array}{l}5.00 \\
\mathrm{E}-07 \\
\end{array}$ & unit & & RoW & $\begin{array}{l}\text { techno } \\
\text { sphere }\end{array}$ \\
\hline $\begin{array}{l}\text { heat, from municipal waste incineration to generic market for } \\
\text { heat district or industrial, other than natural gas }\end{array}$ & $\begin{array}{l}\text { heat, district or industrial, } \\
\text { other than natural gas }\end{array}$ & $\begin{array}{r}5.40 \\
\mathrm{E}+03 \\
\end{array}$ & $\begin{array}{l}\text { megajoul } \\
\mathrm{e}\end{array}$ & & RoW & $\begin{array}{l}\text { techno } \\
\text { sphere }\end{array}$ \\
\hline market for air compressor, screw-type compressor, $300 \mathrm{~kW}$ & $\begin{array}{l}\text { air compressor, screw-type } \\
\text { compressor, } 300 \mathrm{~kW}\end{array}$ & $\begin{array}{l}4.35 \\
\mathrm{E}-06 \\
\end{array}$ & unit & & GLO & $\begin{array}{l}\text { techno } \\
\text { sphere }\end{array}$ \\
\hline market for electricity, low voltage & electricity, low voltage & $\begin{array}{r}5.00 \\
\mathrm{E}+02 \\
\end{array}$ & $\begin{array}{l}\text { kilowatt } \\
\text { hour }\end{array}$ & & $\mathrm{MX}$ & $\begin{array}{l}\text { techno } \\
\text { sphere }\end{array}$ \\
\hline market for electricity, low voltage & electricity, low voltage & $\begin{array}{r}1.14 \\
\mathrm{E}+02 \\
\end{array}$ & $\begin{array}{l}\text { kilowatt } \\
\text { hour }\end{array}$ & & $\mathrm{MX}$ & $\begin{array}{l}\text { techno } \\
\text { sphere }\end{array}$ \\
\hline market for gas turbine, $10 \mathrm{MW}$ electrical & $\begin{array}{l}\text { gas turbine, } 10 \mathrm{MW} \\
\text { electrical }\end{array}$ & $\begin{array}{l}2.54 \\
\mathrm{E}-08\end{array}$ & unit & & GLO & $\begin{array}{l}\text { techno } \\
\text { sphere }\end{array}$ \\
\hline market group for electricity, low voltage & electricity, low voltage & $\begin{array}{r}2.42 \\
\mathrm{E}+01 \\
\end{array}$ & $\begin{array}{l}\text { kilowatt } \\
\text { hour }\end{array}$ & & US & $\begin{array}{l}\text { techno } \\
\text { sphere }\end{array}$ \\
\hline sorbent, generic & & $\begin{array}{r}3.00 \\
\mathrm{E}+00 \\
\end{array}$ & kilogram & & RER & $\begin{array}{l}\text { techno } \\
\text { sphere }\end{array}$ \\
\hline transport, freight, lorry $>32$ metric ton, EURO4 & $\begin{array}{l}\text { transport, freight, lorry } \\
>32 \text { metric ton, EURO4 }\end{array}$ & $\begin{array}{l}2.37 \\
E-01 \\
\end{array}$ & $\begin{array}{l}\text { ton } \\
\text { kilomete } \\
r\end{array}$ & & RoW & $\begin{array}{l}\text { techno } \\
\text { sphere }\end{array}$ \\
\hline transport, freight, lorry $>32$ metric ton, EURO6 & $\begin{array}{l}\text { transport, freight, lorry } \\
>32 \text { metric ton, EURO6 }\end{array}$ & $\begin{array}{l}2.53 \\
\mathrm{E}-01 \\
\end{array}$ & $\begin{array}{l}\text { ton } \\
\text { kilomete } \\
r\end{array}$ & & RoW & $\begin{array}{l}\text { techno } \\
\text { sphere }\end{array}$ \\
\hline transport, freight, sea, container ship & $\begin{array}{l}\text { transport, freight, sea, } \\
\text { container ship }\end{array}$ & $\begin{array}{r}6.42 \\
\mathrm{E}+00\end{array}$ & $\begin{array}{l}\text { ton } \\
\text { kilomete } \\
r\end{array}$ & & GLO & $\begin{array}{l}\text { techno } \\
\text { sphere }\end{array}$ \\
\hline transport, passengers, passenger aircraft, medium haul & $\begin{array}{l}\text { transport, passengers, } \\
\text { aircraft, medium haul }\end{array}$ & $\begin{array}{l}9.96 \\
\mathrm{E}-01 \\
\end{array}$ & $\begin{array}{l}\text { person } \\
\text { kilomete } \\
r\end{array}$ & & GLO & $\begin{array}{l}\text { techno } \\
\text { sphere }\end{array}$ \\
\hline $\begin{array}{l}\text { transport, pipeline, supercritical CO2, } 200 \mathrm{~km} \mathrm{w} \\
\text { recompression }\end{array}$ & & $\begin{array}{r}1.30 \\
\mathrm{E}+03 \\
\end{array}$ & $\begin{array}{l}\text { ton } \\
\text { kilomete } \\
\mathrm{r}\end{array}$ & & RER & $\begin{array}{l}\text { techno } \\
\text { sphere }\end{array}$ \\
\hline
\end{tabular}




\begin{tabular}{|c|c|c|c|c|c|c|}
\hline $\begin{array}{l}\text { transport, pipeline, supercritical CO2, 200km w/o } \\
\text { recompression }\end{array}$ & & $\begin{array}{r}2.00 \\
E+02\end{array}$ & $\begin{array}{l}\text { ton } \\
\text { kilomete } \\
r\end{array}$ & & RER & $\begin{array}{l}\text { techno } \\
\text { sphere }\end{array}$ \\
\hline $\begin{array}{l}\text { carbon dioxide, captured from atmosphere, Waste heat + } \\
\text { PV + Battery, MX }\end{array}$ & & & ton & & $\mathrm{MX}$ & \\
\hline ( & Reference Product & $\begin{array}{l}\text { Amo } \\
\text { unt }\end{array}$ & Unit & Categories & $\begin{array}{l}\text { Loca } \\
\text { tion }\end{array}$ & Type \\
\hline Battery management system, $\mathrm{kWh}$ & & $\begin{array}{l}9.99 \\
\mathrm{E}-02 \\
\end{array}$ & $\begin{array}{l}\text { kilowatt } \\
\text { hour }\end{array}$ & & GLO & $\begin{array}{l}\text { techno } \\
\text { sphere }\end{array}$ \\
\hline Carbon dioxide, fossil & & $\begin{array}{r}2.10 \\
E+01 \\
\end{array}$ & kilogram & $\begin{array}{l}\text { air:urban air } \\
\text { close to ground }\end{array}$ & & $\begin{array}{l}\text { biosph } \\
\text { ere }\end{array}$ \\
\hline Energy management system, kWh & & $\begin{array}{ll}9.99 \\
\mathrm{E}-02\end{array}$ & $\begin{array}{l}\text { kilowatt } \\
\text { hour }\end{array}$ & & GLO & $\begin{array}{l}\text { techno } \\
\text { sphere }\end{array}$ \\
\hline Li-ion (NMC) & & $\begin{array}{l}9.99 \\
\mathrm{E}-02\end{array}$ & $\begin{array}{l}\text { kilowatt } \\
\text { hour }\end{array}$ & & GLO & $\begin{array}{l}\text { techno } \\
\text { sphere }\end{array}$ \\
\hline Power conditioning system, container system & & $\begin{array}{ll}3.00 \\
\mathrm{E}-02\end{array}$ & kW & & GLO & $\begin{array}{l}\text { techno } \\
\text { sphere }\end{array}$ \\
\hline carbon dioxide capture system & & $\begin{array}{l}5.00 \\
\mathrm{E}-07\end{array}$ & unit & & RER & $\begin{array}{l}\text { techno } \\
\text { sphere }\end{array}$ \\
\hline drilling, deep borehole/m & & $\begin{array}{ll}3.38 \\
\mathrm{E}-05 \\
\end{array}$ & meter & & RER & $\begin{array}{l}\text { techno } \\
\text { sphere }\end{array}$ \\
\hline $\begin{array}{l}\text { electricity production, photovoltaic, } 570 \mathrm{kWp} \text { open ground } \\
\text { installation, multi-Si }\end{array}$ & electricity, low voltage & $\begin{array}{r}5.62 \\
E+02 \\
\end{array}$ & $\begin{array}{l}\text { kilowatt } \\
\text { hour }\end{array}$ & & $\mathrm{MX}$ & $\begin{array}{l}\text { techno } \\
\text { sphere }\end{array}$ \\
\hline $\begin{array}{l}\text { electricity production, photovoltaic, } 570 \mathrm{kWp} \text { open ground } \\
\text { installation, multi-Si }\end{array}$ & electricity, low voltage & $\begin{array}{r}1.29 \\
\mathrm{E}+02 \\
\end{array}$ & $\begin{array}{l}\text { kilowatt } \\
\text { hour }\end{array}$ & & $\mathrm{MX}$ & $\begin{array}{l}\text { techno } \\
\text { sphere }\end{array}$ \\
\hline end of life, carbon dioxide capture system & & $\begin{array}{l}5.00 \\
\mathrm{E}-07 \\
\end{array}$ & unit & & RoW & $\begin{array}{l}\text { techno } \\
\text { sphere }\end{array}$ \\
\hline $\begin{array}{l}\text { heat, from municipal waste incineration to generic market for } \\
\text { heat district or industrial, other than natural gas }\end{array}$ & $\begin{array}{l}\text { heat, district or industrial, } \\
\text { other than natural gas }\end{array}$ & $\begin{array}{r}5.40 \\
E+03 \\
\end{array}$ & $\begin{array}{l}\text { megajoul } \\
\mathrm{e}\end{array}$ & & RoW & $\begin{array}{l}\text { techno } \\
\text { sphere }\end{array}$ \\
\hline market for air compressor, screw-type compressor, 300kW & $\begin{array}{l}\text { air compressor, screw-type } \\
\text { compressor, } 300 \mathrm{~kW}\end{array}$ & $\begin{array}{l}4.35 \\
\mathrm{E}-06 \\
\end{array}$ & unit & & GLO & $\begin{array}{l}\text { techno } \\
\text { sphere }\end{array}$ \\
\hline market for gas turbine, $10 \mathrm{MW}$ electrical & $\begin{array}{l}\text { gas turbine, } 10 \mathrm{MW} \\
\text { electrical }\end{array}$ & $\begin{array}{ll}2.54 \\
\mathrm{E}-08 \\
\end{array}$ & unit & & GLO & $\begin{array}{l}\text { techno } \\
\text { sphere }\end{array}$ \\
\hline market group for electricity, low voltage & electricity, low voltage & $\begin{array}{r}2.42 \\
\mathrm{E}+01 \\
\end{array}$ & $\begin{array}{l}\text { kilowatt } \\
\text { hour }\end{array}$ & & US & $\begin{array}{l}\text { techno } \\
\text { sphere }\end{array}$ \\
\hline sorbent, generic & & $\begin{array}{r}3.00 \\
E+00 \\
\end{array}$ & kilogram & & RER & $\begin{array}{l}\text { techno } \\
\text { sphere }\end{array}$ \\
\hline transport, freight, lorry $>32$ metric ton, EURO4 & $\begin{array}{l}\text { transport, freight, lorry } \\
>32 \text { metric ton, EURO4 }\end{array}$ & $\begin{array}{l}2.37 \\
\mathrm{E}-01 \\
\end{array}$ & $\begin{array}{l}\text { ton } \\
\text { kilomete } \\
r\end{array}$ & & RoW & $\begin{array}{l}\text { techno } \\
\text { sphere }\end{array}$ \\
\hline transport, freight, lorry $>32$ metric ton, EURO 6 & $\begin{array}{l}\text { transport, freight, lorry } \\
>32 \text { metric ton, EURO6 }\end{array}$ & $\begin{array}{l}2.53 \\
\mathrm{E}-01\end{array}$ & $\begin{array}{l}\text { ton } \\
\text { kilomete } \\
r\end{array}$ & & RoW & $\begin{array}{l}\text { techno } \\
\text { sphere }\end{array}$ \\
\hline transport, freight, sea, container ship & $\begin{array}{l}\text { transport, freight, sea, } \\
\text { container ship }\end{array}$ & $\begin{array}{r}6.42 \\
E+00\end{array}$ & $\begin{array}{l}\text { ton } \\
\text { kilomete } \\
r\end{array}$ & & GLO & $\begin{array}{l}\text { techno } \\
\text { sphere }\end{array}$ \\
\hline transport, passengers, passenger aircraft, medium haul & $\begin{array}{l}\text { transport, passengers, } \\
\text { aircraft, medium haul }\end{array}$ & $\begin{array}{l}9.96 \\
\mathrm{E}-01\end{array}$ & $\begin{array}{l}\text { person } \\
\text { kilomete } \\
r\end{array}$ & & GLO & $\begin{array}{l}\text { techno } \\
\text { sphere }\end{array}$ \\
\hline $\begin{array}{l}\text { transport, pipeline, supercritical CO2, } 200 \mathrm{~km} \mathrm{w} \\
\text { recompression }\end{array}$ & & $\begin{array}{r}1.30 \\
\mathrm{E}+03\end{array}$ & $\begin{array}{l}\text { ton } \\
\text { kilomete } \\
r\end{array}$ & & RER & $\begin{array}{l}\text { techno } \\
\text { sphere }\end{array}$ \\
\hline $\begin{array}{l}\text { transport, pipeline, supercritical CO2, 200km w/o } \\
\text { recompression }\end{array}$ & & $\begin{array}{r}2.00 \\
E+02 \\
\end{array}$ & $\begin{array}{l}\text { ton } \\
\text { kilomete } \\
r\end{array}$ & & RER & $\begin{array}{l}\text { techno } \\
\text { sphere }\end{array}$ \\
\hline $\begin{array}{l}\text { carbon dioxide, captured from atmosphere, HTHP + Grid, } \\
\text { MX }\end{array}$ & & & ton & & $\mathrm{MX}$ & \\
\hline Name & Reference Product & $\begin{array}{l}\text { Amo } \\
\text { unt }\end{array}$ & Unit & Categories & $\begin{array}{l}\text { Loca } \\
\text { tion }\end{array}$ & Type \\
\hline Carbon dioxide, fossil & & $\begin{array}{r}2.10 \\
E+01 \\
\end{array}$ & kilogram & $\begin{array}{l}\text { air:urban air } \\
\text { close to ground }\end{array}$ & & $\begin{array}{l}\text { biosph } \\
\text { ere }\end{array}$ \\
\hline carbon dioxide capture system & & $\begin{array}{l}5.00 \\
\mathrm{E}-07 \\
\end{array}$ & unit & & RER & $\begin{array}{l}\text { techno } \\
\text { sphere }\end{array}$ \\
\hline drilling, deep borehole/m & & $\begin{array}{ll}3.38 \\
\mathrm{E}-05 \\
\end{array}$ & meter & & RER & $\begin{array}{l}\text { techno } \\
\text { sphere }\end{array}$ \\
\hline end of life, carbon dioxide capture system & & $\begin{array}{ll}5.00 \\
\mathrm{E}-07\end{array}$ & unit & & RoW & $\begin{array}{l}\text { techno } \\
\text { sphere }\end{array}$ \\
\hline heat pump production, brine-water, $\mathrm{CO} 2$ refrigerant, $10 \mathrm{~kW}$ & & $\begin{array}{ll}8.56 \\
\mathrm{E}-04\end{array}$ & unit & & $\mathrm{CH}$ & $\begin{array}{l}\text { techno } \\
\text { sphere }\end{array}$ \\
\hline market for air compressor, screw-type compressor, 300kW & $\begin{array}{l}\text { air compressor, screw-type } \\
\text { compressor, } 300 \mathrm{~kW}\end{array}$ & $\begin{array}{l}4.35 \\
\mathrm{E}-06 \\
\end{array}$ & unit & & GLO & $\begin{array}{l}\text { techno } \\
\text { sphere }\end{array}$ \\
\hline market for electricity, low voltage & electricity, low voltage & $\begin{array}{r}5.00 \\
E+02\end{array}$ & $\begin{array}{l}\text { kilowatt } \\
\text { hour }\end{array}$ & & $\mathrm{MX}$ & $\begin{array}{l}\text { techno } \\
\text { sphere }\end{array}$ \\
\hline market for electricity, low voltage & electricity, low voltage & $\begin{array}{r}1.14 \\
E+02 \\
\end{array}$ & $\begin{array}{l}\text { kilowatt } \\
\text { hour }\end{array}$ & & $\mathrm{MX}$ & $\begin{array}{l}\text { techno } \\
\text { sphere }\end{array}$ \\
\hline
\end{tabular}




\begin{tabular}{|c|c|c|c|c|c|c|}
\hline market for electricity, low voltage & electricity, low voltage & $\begin{array}{r}5.17 \\
E+02 \\
\end{array}$ & $\begin{array}{l}\text { kilowatt } \\
\text { hour }\end{array}$ & & $\mathrm{MX}$ & $\begin{array}{l}\text { techno } \\
\text { sphere }\end{array}$ \\
\hline market for gas turbine, $10 \mathrm{MW}$ electrical & $\begin{array}{l}\text { gas turbine, } 10 \mathrm{MW} \\
\text { electrical }\end{array}$ & $\begin{array}{l}2.54 \\
E-08\end{array}$ & unit & & GLO & $\begin{array}{l}\text { techno } \\
\text { sphere }\end{array}$ \\
\hline market group for electricity, low voltage & electricity, low voltage & $\begin{array}{r}2.42 \\
E+01 \\
\end{array}$ & $\begin{array}{l}\text { kilowatt } \\
\text { hour }\end{array}$ & & US & $\begin{array}{l}\text { techno } \\
\text { sphere }\end{array}$ \\
\hline sorbent, generic & & $\begin{array}{r}3.00 \\
E+00 \\
\end{array}$ & kilogram & & RER & $\begin{array}{l}\text { techno } \\
\text { sphere }\end{array}$ \\
\hline transport, freight, lorry $>32$ metric ton, EURO4 & $\begin{array}{l}\text { transport, freight, lorry } \\
>32 \text { metric ton, EURO4 }\end{array}$ & $\begin{array}{l}2.37 \\
\mathrm{E}-01 \\
\end{array}$ & $\begin{array}{l}\text { ton } \\
\text { kilomete } \\
\mathrm{r}\end{array}$ & & RoW & $\begin{array}{l}\text { techno } \\
\text { sphere }\end{array}$ \\
\hline transport, freight, lorry $>32$ metric ton, EURO 6 & $\begin{array}{l}\text { transport, freight, lorry } \\
>32 \text { metric ton, EURO6 }\end{array}$ & $\begin{array}{l}2.53 \\
\mathrm{E}-01 \\
\end{array}$ & $\begin{array}{l}\text { ton } \\
\text { kilomete } \\
\mathrm{r}\end{array}$ & & RoW & $\begin{array}{l}\text { techno } \\
\text { sphere }\end{array}$ \\
\hline transport, freight, sea, container ship & $\begin{array}{l}\text { transport, freight, sea, } \\
\text { container ship }\end{array}$ & $\begin{array}{r}6.42 \\
\mathrm{E}+00 \\
\end{array}$ & $\begin{array}{l}\text { ton } \\
\text { kilomete } \\
\mathrm{r}\end{array}$ & & GLO & $\begin{array}{l}\text { techno } \\
\text { sphere }\end{array}$ \\
\hline transport, passengers, passenger aircraft, medium haul & $\begin{array}{l}\text { transport, passengers, } \\
\text { aircraft, medium haul }\end{array}$ & $\begin{array}{l}9.96 \\
\mathrm{E}-01 \\
\end{array}$ & $\begin{array}{l}\text { person } \\
\text { kilomete } \\
r\end{array}$ & & GLO & $\begin{array}{l}\text { techno } \\
\text { sphere }\end{array}$ \\
\hline $\begin{array}{l}\text { transport, pipeline, supercritical CO2, } 200 \mathrm{~km} \mathrm{w} \\
\text { recompression }\end{array}$ & & $\begin{array}{r}1.30 \\
E+03 \\
\end{array}$ & $\begin{array}{l}\text { ton } \\
\text { kilomete } \\
\mathrm{r}\end{array}$ & & RER & $\begin{array}{l}\text { techno } \\
\text { sphere }\end{array}$ \\
\hline $\begin{array}{l}\text { transport, pipeline, supercritical } \mathrm{CO} 2,200 \mathrm{~km} \text { w/o } \\
\text { recompression }\end{array}$ & & $\begin{array}{r}2.00 \\
\mathrm{E}+02 \\
\end{array}$ & $\begin{array}{l}\text { ton } \\
\text { kilomete } \\
\mathrm{r}\end{array}$ & & RER & $\begin{array}{l}\text { techno } \\
\text { sphere }\end{array}$ \\
\hline $\begin{array}{l}\text { carbon dioxide, captured from atmosphere, Autonomous } \\
\text { Fresnel + PV, MX }\end{array}$ & & & ton & & $\mathrm{MX}$ & \\
\hline Name & Reference Product & $\begin{array}{l}\text { Amo } \\
\text { unt }\end{array}$ & Unit & Categories & $\begin{array}{l}\text { Loca } \\
\text { tion }\end{array}$ & Type \\
\hline Battery management system, kWh & & $\begin{array}{ll}1.04 \\
E-01 \\
\end{array}$ & $\begin{array}{l}\text { kilowatt } \\
\text { hour }\end{array}$ & & GLO & $\begin{array}{l}\text { techno } \\
\text { sphere }\end{array}$ \\
\hline Carbon dioxide, fossil & & $\begin{array}{r}2.10 \\
E+01 \\
\end{array}$ & kilogram & $\begin{array}{l}\text { air:urban air } \\
\text { close to ground }\end{array}$ & & $\begin{array}{l}\text { biosph } \\
\text { ere }\end{array}$ \\
\hline Energy management system, kWh & & $\begin{array}{l}1.04 \\
\mathrm{E}-01 \\
\end{array}$ & $\begin{array}{l}\text { kilowatt } \\
\text { hour }\end{array}$ & & GLO & $\begin{array}{l}\text { techno } \\
\text { sphere }\end{array}$ \\
\hline Li-ion (NMC) & & $\begin{array}{l}1.04 \\
\mathrm{E}-01 \\
\end{array}$ & $\begin{array}{l}\text { kilowatt } \\
\text { hour }\end{array}$ & & GLO & $\begin{array}{l}\text { techno } \\
\text { sphere }\end{array}$ \\
\hline Power conditioning system, container system & & $\begin{array}{l}3.13 \\
\mathrm{E}-02 \\
\end{array}$ & $\mathrm{~kW}$ & & GLO & $\begin{array}{l}\text { techno } \\
\text { sphere }\end{array}$ \\
\hline carbon dioxide capture system & & $\begin{array}{l}5.00 \\
\mathrm{E}-07 \\
\end{array}$ & unit & & RER & $\begin{array}{l}\text { techno } \\
\text { sphere }\end{array}$ \\
\hline drilling, deep borehole/m & & $\begin{array}{l}3.38 \\
\mathrm{E}-05 \\
\end{array}$ & meter & & RER & $\begin{array}{l}\text { techno } \\
\text { sphere }\end{array}$ \\
\hline $\begin{array}{l}\text { electricity production, photovoltaic, } 570 \mathrm{kWp} \text { open ground } \\
\text { installation, multi-Si }\end{array}$ & electricity, low voltage & $\begin{array}{r}5.62 \\
\mathrm{E}+02 \\
\end{array}$ & $\begin{array}{l}\text { kilowatt } \\
\text { hour }\end{array}$ & & $\mathrm{MX}$ & $\begin{array}{l}\text { techno } \\
\text { sphere }\end{array}$ \\
\hline $\begin{array}{l}\text { electricity production, photovoltaic, } 570 \mathrm{kWp} \text { open ground } \\
\text { installation, multi-Si }\end{array}$ & electricity, low voltage & $\begin{array}{r}1.29 \\
\mathrm{E}+02 \\
\end{array}$ & $\begin{array}{l}\text { kilowatt } \\
\text { hour }\end{array}$ & & $\mathrm{MX}$ & $\begin{array}{l}\text { techno } \\
\text { sphere }\end{array}$ \\
\hline end of life, carbon dioxide capture system & & $\begin{array}{l}5.00 \\
\mathrm{E}-07 \\
\end{array}$ & unit & & RoW & $\begin{array}{l}\text { techno } \\
\text { sphere }\end{array}$ \\
\hline $\begin{array}{l}\text { heat production, Fresnel solar collector plant, San Luis } \\
\text { Potoso, Mexico }\end{array}$ & $\begin{array}{l}\text { heat, central or small- } \\
\text { scale, Fresnel plant }\end{array}$ & $\begin{array}{r}5.40 \\
\mathrm{E}+03 \\
\end{array}$ & $\begin{array}{l}\text { megajoul } \\
\mathrm{e}\end{array}$ & & $\mathrm{MX}$ & $\begin{array}{l}\text { techno } \\
\text { sphere }\end{array}$ \\
\hline market for air compressor, screw-type compressor, $300 \mathrm{~kW}$ & $\begin{array}{l}\text { air compressor, screw-type } \\
\text { compressor, } 300 \mathrm{~kW}\end{array}$ & $\begin{array}{l}4.35 \\
\mathrm{E}-06 \\
\end{array}$ & unit & & GLO & $\begin{array}{l}\text { techno } \\
\text { sphere }\end{array}$ \\
\hline market for gas turbine, $10 \mathrm{MW}$ electrical & $\begin{array}{l}\text { gas turbine, 10MW } \\
\text { electrical }\end{array}$ & $\begin{array}{ll}2.54 \\
\mathrm{E}-08 \\
\end{array}$ & unit & & GLO & $\begin{array}{l}\text { techno } \\
\text { sphere }\end{array}$ \\
\hline market group for electricity, low voltage & electricity, low voltage & $\begin{array}{r}2.42 \\
E+01 \\
\end{array}$ & $\begin{array}{l}\text { kilowatt } \\
\text { hour }\end{array}$ & & US & $\begin{array}{l}\text { techno } \\
\text { sphere }\end{array}$ \\
\hline sorbent, generic & & $\begin{array}{r}3.00 \\
E+00 \\
\end{array}$ & kilogram & & RER & $\begin{array}{l}\text { techno } \\
\text { sphere }\end{array}$ \\
\hline storage unit production, Fresnel plant, Jordan, $12 \mathrm{~h}$ storage & $\begin{array}{l}\text { storage unit, Fresnel plant, } \\
\text { Jordan, } 12 \mathrm{~h} \text { storage }\end{array}$ & $\begin{array}{l}4.00 \\
\mathrm{E}-07 \\
\end{array}$ & unit & & $\mathrm{JO}$ & $\begin{array}{l}\text { techno } \\
\text { sphere }\end{array}$ \\
\hline transport, freight, lorry $>32$ metric ton, EURO4 & $\begin{array}{l}\text { transport, freight, lorry } \\
>32 \text { metric ton, EURO4 }\end{array}$ & $\begin{array}{l}2.37 \\
\mathrm{E}-01 \\
\end{array}$ & $\begin{array}{l}\text { ton } \\
\text { kilomete } \\
r\end{array}$ & & RoW & $\begin{array}{l}\text { techno } \\
\text { sphere }\end{array}$ \\
\hline transport, freight, lorry $>32$ metric ton, EURO 6 & $\begin{array}{l}\text { transport, freight, lorry } \\
>32 \text { metric ton, EURO6 }\end{array}$ & $\begin{array}{l}2.53 \\
\mathrm{E}-01 \\
\end{array}$ & $\begin{array}{l}\text { ton } \\
\text { kilomete } \\
\mathrm{r}\end{array}$ & & RoW & $\begin{array}{l}\text { techno } \\
\text { sphere } \\
\end{array}$ \\
\hline transport, freight, sea, container ship & $\begin{array}{l}\text { transport, freight, sea, } \\
\text { container ship }\end{array}$ & $\begin{array}{r}6.42 \\
E+00 \\
\end{array}$ & $\begin{array}{l}\text { ton } \\
\text { kilomete } \\
\mathrm{r}\end{array}$ & & GLO & $\begin{array}{l}\text { techno } \\
\text { sphere }\end{array}$ \\
\hline transport, passengers, passenger aircraft, medium haul & $\begin{array}{l}\text { transport, passengers, } \\
\text { aircraft, medium haul }\end{array}$ & $\begin{array}{l}9.96 \\
\mathrm{E}-01 \\
\end{array}$ & $\begin{array}{l}\text { person } \\
\text { kilomete } \\
r\end{array}$ & & GLO & $\begin{array}{l}\text { techno } \\
\text { sphere }\end{array}$ \\
\hline $\begin{array}{l}\text { transport, pipeline, supercritical CO2, } 200 \mathrm{~km} \mathrm{w} \\
\text { recompression }\end{array}$ & & $\begin{array}{r}1.30 \\
\mathrm{E}+03 \\
\end{array}$ & $\begin{array}{l}\text { ton } \\
\text { kilomete } \\
\mathrm{r}\end{array}$ & & RER & $\begin{array}{l}\text { techno } \\
\text { sphere }\end{array}$ \\
\hline
\end{tabular}




\begin{tabular}{|c|c|c|c|c|c|c|}
\hline $\begin{array}{l}\text { transport, pipeline, supercritical CO2, 200km w/o } \\
\text { recompression }\end{array}$ & & $\begin{array}{r}2.00 \\
E+02\end{array}$ & $\begin{array}{l}\text { ton } \\
\text { kilomete } \\
r\end{array}$ & & RER & $\begin{array}{l}\text { techno } \\
\text { sphere }\end{array}$ \\
\hline $\begin{array}{l}\text { carbon dioxide, captured from atmosphere, Autonomous } \\
\text { HTHP + PV, MX }\end{array}$ & & & ton & & $\mathrm{MX}$ & \\
\hline ( & Reference Product & $\begin{array}{l}\text { Amo } \\
\text { unt }\end{array}$ & Unit & Categories & $\begin{array}{l}\text { Loca } \\
\text { tion }\end{array}$ & Type \\
\hline Battery management system, $\mathrm{kWh}$ & & $\begin{array}{l}1.84 \\
\mathrm{E}-01 \\
\end{array}$ & $\begin{array}{l}\text { kilowatt } \\
\text { hour }\end{array}$ & & GLO & $\begin{array}{l}\text { techno } \\
\text { sphere }\end{array}$ \\
\hline Carbon dioxide, fossil & & $\begin{array}{r}2.10 \\
E+01 \\
\end{array}$ & kilogram & $\begin{array}{l}\text { air:urban air } \\
\text { close to ground }\end{array}$ & & $\begin{array}{l}\text { biosph } \\
\text { ere }\end{array}$ \\
\hline Energy management system, kWh & & $\begin{array}{ll}1.84 \\
\mathrm{E}-01\end{array}$ & $\begin{array}{l}\text { kilowatt } \\
\text { hour }\end{array}$ & & GLO & $\begin{array}{l}\text { techno } \\
\text { sphere }\end{array}$ \\
\hline Li-ion (NMC) & & $\begin{array}{l}1.84 \\
\mathrm{E}-01\end{array}$ & $\begin{array}{l}\text { kilowatt } \\
\text { hour }\end{array}$ & & GLO & $\begin{array}{l}\text { techno } \\
\text { sphere }\end{array}$ \\
\hline Power conditioning system, container system & & $\begin{array}{l}5.52 \\
\mathrm{E}-02 \\
\end{array}$ & kW & & GLO & $\begin{array}{l}\text { techno } \\
\text { sphere }\end{array}$ \\
\hline carbon dioxide capture system & & $\begin{array}{l}5.00 \\
\mathrm{E}-07\end{array}$ & unit & & RER & $\begin{array}{l}\text { techno } \\
\text { sphere }\end{array}$ \\
\hline drilling, deep borehole/m & & $\begin{array}{ll}3.38 \\
\mathrm{E}-05 \\
\end{array}$ & meter & & RER & $\begin{array}{l}\text { techno } \\
\text { sphere }\end{array}$ \\
\hline $\begin{array}{l}\text { electricity production, photovoltaic, } 570 \mathrm{kWp} \text { open ground } \\
\text { installation, multi-Si }\end{array}$ & electricity, low voltage & $\begin{array}{r}5.62 \\
E+02 \\
\end{array}$ & $\begin{array}{l}\text { kilowatt } \\
\text { hour }\end{array}$ & & $\mathrm{MX}$ & $\begin{array}{l}\text { techno } \\
\text { sphere }\end{array}$ \\
\hline $\begin{array}{l}\text { electricity production, photovoltaic, } 570 \mathrm{kWp} \text { open ground } \\
\text { installation, multi-Si }\end{array}$ & electricity, low voltage & $\begin{array}{r}1.29 \\
\mathrm{E}+02 \\
\end{array}$ & $\begin{array}{l}\text { kilowatt } \\
\text { hour }\end{array}$ & & $\mathrm{MX}$ & $\begin{array}{l}\text { techno } \\
\text { sphere }\end{array}$ \\
\hline $\begin{array}{l}\text { electricity production, photovoltaic, } 570 \mathrm{kWp} \text { open ground } \\
\text { installation, multi-Si }\end{array}$ & electricity, low voltage & $\begin{array}{r}5.81 \\
E+02 \\
\end{array}$ & $\begin{array}{l}\text { kilowatt } \\
\text { hour }\end{array}$ & & $\mathrm{MX}$ & $\begin{array}{l}\text { techno } \\
\text { sphere }\end{array}$ \\
\hline end of life, carbon dioxide capture system & & $\begin{array}{l}5.00 \\
\mathrm{E}-07 \\
\end{array}$ & unit & & RoW & $\begin{array}{l}\text { techno } \\
\text { sphere }\end{array}$ \\
\hline heat pump production, brine-water, $\mathrm{CO} 2$ refrigerant, $10 \mathrm{~kW}$ & & $\begin{array}{l}8.56 \\
\mathrm{E}-04 \\
\end{array}$ & unit & & $\mathrm{CH}$ & $\begin{array}{l}\text { techno } \\
\text { sphere }\end{array}$ \\
\hline market for air compressor, screw-type compressor, 300kW & $\begin{array}{l}\text { air compressor, screw-type } \\
\text { compressor, } 300 \mathrm{~kW}\end{array}$ & $\begin{array}{ll}4.35 \\
\mathrm{E}-06\end{array}$ & unit & & GLO & $\begin{array}{l}\text { techno } \\
\text { sphere }\end{array}$ \\
\hline market for gas turbine, $10 \mathrm{MW}$ electrical & $\begin{array}{l}\text { gas turbine, 10MW } \\
\text { electrical }\end{array}$ & $\begin{array}{ll}2.54 \\
\mathrm{E}-08 \\
\end{array}$ & unit & & GLO & $\begin{array}{l}\text { techno } \\
\text { sphere }\end{array}$ \\
\hline market group for electricity, low voltage & electricity, low voltage & $\begin{array}{r}2.42 \\
E+01 \\
\end{array}$ & $\begin{array}{l}\text { kilowatt } \\
\text { hour }\end{array}$ & & US & $\begin{array}{l}\text { techno } \\
\text { sphere }\end{array}$ \\
\hline sorbent, generic & & $\begin{array}{r}3.00 \\
E+00 \\
\end{array}$ & kilogram & & RER & $\begin{array}{l}\text { techno } \\
\text { sphere }\end{array}$ \\
\hline transport, freight, lorry $>32$ metric ton, EURO 4 & $\begin{array}{l}\text { transport, freight, lorry } \\
>32 \text { metric ton, EURO4 }\end{array}$ & $\begin{array}{l}2.37 \\
\mathrm{E}-01\end{array}$ & $\begin{array}{l}\text { ton } \\
\text { kilomete } \\
r\end{array}$ & & RoW & $\begin{array}{l}\text { techno } \\
\text { sphere }\end{array}$ \\
\hline transport, freight, lorry $>32$ metric ton, EURO 6 & $\begin{array}{l}\text { transport, freight, lorry } \\
>32 \text { metric ton, EURO6 }\end{array}$ & $\begin{array}{l}2.53 \\
\mathrm{E}-01 \\
\end{array}$ & $\begin{array}{l}\text { ton } \\
\text { kilomete } \\
\mathrm{r}\end{array}$ & & RoW & $\begin{array}{l}\text { techno } \\
\text { sphere }\end{array}$ \\
\hline transport, freight, sea, container ship & $\begin{array}{l}\text { transport, freight, sea, } \\
\text { container ship }\end{array}$ & $\begin{array}{r}6.42 \\
E+00 \\
\end{array}$ & $\begin{array}{l}\text { ton } \\
\text { kilomete } \\
r\end{array}$ & & GLO & $\begin{array}{l}\text { techno } \\
\text { sphere }\end{array}$ \\
\hline transport, passengers, passenger aircraft, medium haul & $\begin{array}{l}\text { transport, passengers, } \\
\text { aircraft, medium haul }\end{array}$ & $\begin{array}{l}9.96 \\
\mathrm{E}-01 \\
\end{array}$ & $\begin{array}{l}\text { person } \\
\text { kilomete } \\
r\end{array}$ & & GLO & $\begin{array}{l}\text { techno } \\
\text { sphere }\end{array}$ \\
\hline $\begin{array}{l}\text { transport, pipeline, supercritical CO2, } 200 \mathrm{~km} \mathrm{w} \\
\text { recompression }\end{array}$ & & $\begin{array}{r}1.30 \\
\mathrm{E}+03 \\
\end{array}$ & $\begin{array}{l}\text { ton } \\
\text { kilomete } \\
r\end{array}$ & & RER & $\begin{array}{l}\text { techno } \\
\text { sphere }\end{array}$ \\
\hline $\begin{array}{l}\text { transport, pipeline, supercritical CO2, 200km w/o } \\
\text { recompression }\end{array}$ & & $\begin{array}{r}2.00 \\
E+02\end{array}$ & $\begin{array}{l}\text { ton } \\
\text { kilomete } \\
r\end{array}$ & & RER & $\begin{array}{l}\text { techno } \\
\text { sphere }\end{array}$ \\
\hline $\begin{array}{l}\text { carbon dioxide, captured from atmosphere, Waste heat + } \\
\text { Grid, IS }\end{array}$ & & & ton & & IS & \\
\hline Name & Reference Product & $\begin{array}{l}\text { Amo } \\
\text { unt }\end{array}$ & Unit & Categories & $\begin{array}{l}\text { Loca } \\
\text { tion }\end{array}$ & Type \\
\hline Carbon dioxide, fossil & & $\begin{array}{r}1.40 \\
E+00 \\
\end{array}$ & kilogram & $\begin{array}{l}\text { air:urban air } \\
\text { close to ground }\end{array}$ & & $\begin{array}{l}\text { biosph } \\
\text { ere }\end{array}$ \\
\hline carbon dioxide capture system & & $\begin{array}{l}5.00 \\
\mathrm{E}-07 \\
\end{array}$ & unit & & RER & $\begin{array}{l}\text { techno } \\
\text { sphere }\end{array}$ \\
\hline drilling, deep borehole/m & & $\begin{array}{ll}3.38 \\
\mathrm{E}-05 \\
\end{array}$ & meter & & RER & $\begin{array}{l}\text { techno } \\
\text { sphere }\end{array}$ \\
\hline end of life, carbon dioxide capture system & & $\begin{array}{ll}5.00 \\
\mathrm{E}-07 \\
\end{array}$ & unit & & RoW & $\begin{array}{l}\text { techno } \\
\text { sphere }\end{array}$ \\
\hline $\begin{array}{l}\text { heat, from municipal waste incineration to generic market for } \\
\text { heat district or industrial, other than natural gas }\end{array}$ & $\begin{array}{l}\text { heat, district or industrial, } \\
\text { other than natural gas }\end{array}$ & $\begin{array}{r}5.40 \\
\mathrm{E}+03 \\
\end{array}$ & $\begin{array}{l}\text { megajoul } \\
\mathrm{e}\end{array}$ & & RoW & $\begin{array}{l}\text { techno } \\
\text { sphere }\end{array}$ \\
\hline market for air compressor, screw-type compressor, 300kW & $\begin{array}{l}\text { air compressor, screw-type } \\
\text { compressor, } 300 \mathrm{~kW}\end{array}$ & $\begin{array}{l}4.35 \\
\mathrm{E}-06\end{array}$ & unit & & GLO & $\begin{array}{l}\text { techno } \\
\text { sphere }\end{array}$ \\
\hline market for electricity, low voltage & electricity, low voltage & $\begin{array}{r}5.00 \\
E+02 \\
\end{array}$ & $\begin{array}{l}\text { kilowatt } \\
\text { hour }\end{array}$ & & IS & $\begin{array}{l}\text { techno } \\
\text { sphere }\end{array}$ \\
\hline
\end{tabular}




\begin{tabular}{|c|c|c|c|c|c|c|}
\hline market for electricity, low voltage & electricity, low voltage & $\begin{array}{r}1.14 \\
\mathrm{E}+02\end{array}$ & $\begin{array}{l}\text { kilowatt } \\
\text { hour }\end{array}$ & & IS & $\begin{array}{l}\text { techno } \\
\text { sphere }\end{array}$ \\
\hline market for electricity, low voltage & electricity, low voltage & $\begin{array}{r}2.42 \\
\mathrm{E}+01 \\
\end{array}$ & $\begin{array}{l}\text { kilowatt } \\
\text { hour }\end{array}$ & & IS & $\begin{array}{l}\text { techno } \\
\text { sphere }\end{array}$ \\
\hline market for gas turbine, $10 \mathrm{MW}$ electrical & $\begin{array}{l}\text { gas turbine, } 10 \mathrm{MW} \\
\text { electrical }\end{array}$ & $\begin{array}{l}2.54 \\
E-08\end{array}$ & unit & & GLO & $\begin{array}{l}\text { techno } \\
\text { sphere }\end{array}$ \\
\hline sorbent, generic & & $\begin{array}{r}3.00 \\
E+00\end{array}$ & kilogram & & RER & $\begin{array}{l}\text { techno } \\
\text { sphere }\end{array}$ \\
\hline transport, freight, lorry $>32$ metric ton, EURO 6 & $\begin{array}{l}\text { transport, freight, lorry } \\
>32 \text { metric ton, EURO6 }\end{array}$ & $\begin{array}{l}2.53 \\
E-01\end{array}$ & $\begin{array}{l}\text { ton } \\
\text { kilomete } \\
r\end{array}$ & & RoW & $\begin{array}{l}\text { techno } \\
\text { sphere }\end{array}$ \\
\hline transport, freight, sea, container ship & $\begin{array}{l}\text { transport, freight, sea, } \\
\text { container ship }\end{array}$ & $\begin{array}{r}3.12 \\
\mathrm{E}+00 \\
\end{array}$ & $\begin{array}{l}\text { ton } \\
\text { kilomete } \\
\mathrm{r}\end{array}$ & & GLO & $\begin{array}{l}\text { techno } \\
\text { sphere }\end{array}$ \\
\hline transport, passengers, passenger aircraft, medium haul & $\begin{array}{l}\text { transport, passengers, } \\
\text { aircraft, medium haul }\end{array}$ & $\begin{array}{l}2.73 \\
E-01\end{array}$ & $\begin{array}{l}\text { person } \\
\text { kilomete } \\
r\end{array}$ & & GLO & $\begin{array}{l}\text { techno } \\
\text { sphere }\end{array}$ \\
\hline $\begin{array}{l}\text { transport, pipeline, supercritical CO2, 200km w/o } \\
\text { recompression }\end{array}$ & & $\begin{array}{r}1.00 \\
\mathrm{E}+02 \\
\end{array}$ & $\begin{array}{l}\text { ton } \\
\text { kilomete } \\
\mathrm{r}\end{array}$ & & RER & $\begin{array}{l}\text { techno } \\
\text { sphere }\end{array}$ \\
\hline carbon dioxide, captured from atmosphere, HTHP + Grid, IS & & & ton & & IS & \\
\hline Name & Reference Product & $\begin{array}{l}\text { Amo } \\
\text { unt }\end{array}$ & Unit & Categories & $\begin{array}{l}\text { Loca } \\
\text { tion }\end{array}$ & Type \\
\hline Carbon dioxide, fossil & & $\begin{array}{r}1.40 \\
\mathrm{E}+00\end{array}$ & kilogram & $\begin{array}{l}\text { air:urban air } \\
\text { close to ground }\end{array}$ & & $\begin{array}{l}\text { biosph } \\
\text { ere }\end{array}$ \\
\hline carbon dioxide capture system & & $\begin{array}{l}5.00 \\
\mathrm{E}-07\end{array}$ & unit & & RER & $\begin{array}{l}\text { techno } \\
\text { sphere }\end{array}$ \\
\hline drilling, deep borehole/m & & $\begin{array}{l}3.38 \\
E-05 \\
\end{array}$ & meter & & RER & $\begin{array}{l}\text { techno } \\
\text { sphere }\end{array}$ \\
\hline end of life, carbon dioxide capture system & & $\begin{array}{l}5.00 \\
\mathrm{E}-07 \\
\end{array}$ & unit & & RoW & $\begin{array}{l}\text { techno } \\
\text { sphere }\end{array}$ \\
\hline heat pump production, brine-water, $\mathrm{CO} 2$ refrigerant, $10 \mathrm{~kW}$ & & $\begin{array}{l}8.56 \\
\mathrm{E}-04 \\
\end{array}$ & unit & & $\mathrm{CH}$ & $\begin{array}{l}\text { techno } \\
\text { sphere }\end{array}$ \\
\hline market for air compressor, screw-type compressor, 300kW & $\begin{array}{l}\text { air compressor, screw-type } \\
\text { compressor, } 300 \mathrm{~kW}\end{array}$ & $\begin{array}{l}4.35 \\
E-06 \\
\end{array}$ & unit & & GLO & $\begin{array}{l}\text { techno } \\
\text { sphere }\end{array}$ \\
\hline market for electricity, low voltage & electricity, low voltage & $\begin{array}{r}5.00 \\
E+02 \\
\end{array}$ & $\begin{array}{l}\text { kilowatt } \\
\text { hour }\end{array}$ & & IS & $\begin{array}{l}\text { techno } \\
\text { sphere }\end{array}$ \\
\hline market for electricity, low voltage & electricity, low voltage & $\begin{array}{r}1.14 \\
\mathrm{E}+02 \\
\end{array}$ & $\begin{array}{l}\text { kilowatt } \\
\text { hour }\end{array}$ & & IS & $\begin{array}{l}\text { techno } \\
\text { sphere }\end{array}$ \\
\hline market for electricity, low voltage & electricity, low voltage & $\begin{array}{r}5.17 \\
\mathrm{E}+02 \\
\end{array}$ & $\begin{array}{l}\text { kilowatt } \\
\text { hour }\end{array}$ & & IS & $\begin{array}{l}\text { techno } \\
\text { sphere }\end{array}$ \\
\hline market for electricity, low voltage & electricity, low voltage & $\begin{array}{r}2.42 \\
E+01\end{array}$ & $\begin{array}{l}\text { kilowatt } \\
\text { hour }\end{array}$ & & IS & $\begin{array}{l}\text { techno } \\
\text { sphere }\end{array}$ \\
\hline market for gas turbine, $10 \mathrm{MW}$ electrical & $\begin{array}{l}\text { gas turbine, 10MW } \\
\text { electrical }\end{array}$ & $\begin{array}{l}2.54 \\
\mathrm{E}-08 \\
\end{array}$ & unit & & GLO & $\begin{array}{l}\text { techno } \\
\text { sphere }\end{array}$ \\
\hline sorbent, generic & & $\begin{array}{r}3.00 \\
E+00 \\
\end{array}$ & kilogram & & RER & $\begin{array}{l}\text { techno } \\
\text { sphere }\end{array}$ \\
\hline transport, freight, lorry $>32$ metric ton, EURO 6 & $\begin{array}{l}\text { transport, freight, lorry } \\
>32 \text { metric ton, EURO6 }\end{array}$ & $\begin{array}{l}2.53 \\
\mathrm{E}-01 \\
\end{array}$ & $\begin{array}{l}\text { ton } \\
\text { kilomete } \\
\mathrm{r} \\
\end{array}$ & & RoW & $\begin{array}{l}\text { techno } \\
\text { sphere }\end{array}$ \\
\hline transport, freight, sea, container ship & $\begin{array}{l}\text { transport, freight, sea, } \\
\text { container ship }\end{array}$ & $\begin{array}{r}3.12 \\
\mathrm{E}+00 \\
\end{array}$ & $\begin{array}{l}\text { ton } \\
\text { kilomete } \\
r\end{array}$ & & GLO & $\begin{array}{l}\text { techno } \\
\text { sphere }\end{array}$ \\
\hline transport, passengers, passenger aircraft, medium haul & $\begin{array}{l}\text { transport, passengers, } \\
\text { aircraft, medium haul }\end{array}$ & $\begin{array}{l}2.73 \\
E-01\end{array}$ & $\begin{array}{l}\text { person } \\
\text { kilomete } \\
r\end{array}$ & & GLO & $\begin{array}{l}\text { techno } \\
\text { sphere }\end{array}$ \\
\hline $\begin{array}{l}\text { transport, pipeline, supercritical CO2, } 200 \mathrm{~km} \text { w/o } \\
\text { recompression }\end{array}$ & & $\begin{array}{r}1.00 \\
E+02\end{array}$ & $\begin{array}{l}\text { ton } \\
\text { kilomete } \\
r\end{array}$ & & RER & $\begin{array}{l}\text { techno } \\
\text { sphere }\end{array}$ \\
\hline
\end{tabular}




\section{S6. LCA results: all environmental impact categories}

Table 8 presents the full results for all system layouts on all environmental impact categories. Red shaded cells represent a high environmental impact for the specific environmental impact category compared to other system layouts, light blue means an average environmental impact for the specific environmental impact category compared to other system layouts, while dark blue means a low environmental impact for the specific environmental impact category compared to other system layouts.

Table 8. LCA results for all environmental impact categories for all DACCS alternatives per FU "Gross removal of 1 ton $\mathrm{CO}_{2}$ from the atmosphere".

\begin{tabular}{|c|c|c|c|c|c|c|c|c|c|c|c|c|c|c|c|c|c|}
\hline & $\begin{array}{l}\text { Land } \\
\text { transform } \\
\text { ation }\end{array}$ & $\begin{array}{c}\text { carcinoge } \\
\text { nic } \\
\text { effects }\end{array}$ & 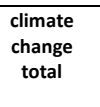 & fossils & $\begin{array}{l}\text { freshwater and } \\
\text { terrestrial } \\
\text { acidification }\end{array}$ & $\begin{array}{l}\text { freshwater } \\
\text { ecotoxicity }\end{array}$ & $\begin{array}{l}\text { freshwater } \\
\text { eutrophicati } \\
\text { on }\end{array}$ & $\begin{array}{c}\text { ionising } \\
\text { radiatio } \\
n\end{array}$ & $\begin{array}{l}\text { land } \\
\text { use }\end{array}$ & $\begin{array}{c}\text { marine } \\
\text { eutrop } \\
\text { hicatio } \\
n\end{array}$ & $\begin{array}{c}\text { minerals } \\
\text { and } \\
\text { metals }\end{array}$ & $\begin{array}{c}\text { non- } \\
\text { carcinogeni } \\
\text { c effects }\end{array}$ & $\begin{array}{c}\begin{array}{c}\text { ozone } \\
\text { layer } \\
\text { depletion }\end{array} \\
\end{array}$ & $\begin{array}{l}\text { photochemical } \\
\text { ozone creation }\end{array}$ & $\begin{array}{c}\begin{array}{c}\text { respiratory } \\
\text { effects, } \\
\text { inorganics }\end{array}\end{array}$ & $\begin{array}{c}\text { terrestrial } \\
\text { eutrophicati } \\
\text { on }\end{array}$ & $\begin{array}{c}\text { water } \\
\text { consump } \\
\text { tion }\end{array}$ \\
\hline Unit & $\mathrm{m}^{2}$ & CTUh & $\begin{array}{l}\mathrm{kg} \mathrm{CO}_{2-} \\
\mathrm{Eq} .\end{array}$ & $\begin{array}{c}\text { megajou } \\
\text { le }\end{array}$ & mol H+Eq. & стU & kg P-Eq. & $\begin{array}{c}\text { kg } \\
\text { U235- } \\
\text { Eq. }\end{array}$ & $\begin{array}{c}\text { poin } \\
\text { ts }\end{array}$ & $\begin{array}{l}\text { kg N- } \\
\text { Eq. }\end{array}$ & kg Sb-Eq. & cTUh & kg CFC-11. & kg NMVOC-. & disease i. & mol N-Eq. & $\mathrm{m}^{3}$-eq. \\
\hline $\begin{array}{c}\text { Autonomous } \\
\text { Fresnel + PV, } \\
\mathrm{CL}\end{array}$ & 7.16E-01 & $6.03 \mathrm{E}-06$ & $1.25 \mathrm{E}+02$ & $\begin{array}{r}1.69 E+0 \\
3\end{array}$ & $9.29 \mathrm{E}-01$ & $1.57 \mathrm{E}+02$ & 7.40E-02 & $\begin{array}{r}1.75 E+0 \\
1\end{array}$ & $\begin{array}{r}4.24 \\
E+0 \\
3\end{array}$ & $\begin{array}{r}1.30 \mathrm{E}- \\
01\end{array}$ & $1.09 \mathrm{E}-02$ & 3.72E-05 & $9.61 \mathrm{E}-06$ & $4.24 \mathrm{E}-01$ & $6.38 \mathrm{E}-06$ & $1.36 \mathrm{E}+00$ & $2.30 E+00$ \\
\hline $\begin{array}{c}\text { Autonomous } \\
\text { Fresnel + PV, } \\
\text { ES }\end{array}$ & $9.66 \mathrm{E}-01$ & $6.34 \mathrm{E}-06$ & $1.09 \mathrm{E}+02$ & $\begin{array}{r}1.64 \mathrm{E}+0 \\
3\end{array}$ & $9.46 \mathrm{E}-01$ & $1.72 E+02$ & $6.94 \mathrm{E}-02$ & $\begin{array}{r}1.49 E+0 \\
1\end{array}$ & $\begin{array}{r}6.23 \\
E+0 \\
3\end{array}$ & $\begin{array}{r}1.24 E- \\
01\end{array}$ & $1.38 \mathrm{E}-02$ & 4.05E-05 & $9.36 \mathrm{E}-06$ & $4.25 \mathrm{E}-01$ & $6.50 \mathrm{E}-06$ & $1.30 \mathrm{E}+00$ & $2.06 \mathrm{E}+00$ \\
\hline $\begin{array}{l}\text { Autonomous } \\
\text { Fresnel + PV, }\end{array}$ & & & & $1.56 \mathrm{E}+0$ & & & & $8.84 E+0$ & $\begin{array}{r}4.71 \\
E+0\end{array}$ & $1.18 \mathrm{E}-$ & & & & & & & \\
\hline GR & $7.51 \mathrm{E}-01$ & $5.99 \mathrm{E}-06$ & $1.08 \mathrm{E}+02$ & 3 & $9.21 \mathrm{E}-01$ & $1.61 \mathrm{E}+02$ & $9.99 \mathrm{E}-02$ & & 3 & 01 & $1.19 \mathrm{E}-02$ & $3.77 \mathrm{E}-05$ & $8.91 E-06$ & $3.84 \mathrm{E}-01$ & $5.81 \mathrm{E}-06$ & $1.17 E+00$ & $1.72 E+00$ \\
\hline $\begin{array}{c}\text { Autonomous } \\
\text { Fresnel + PV, } \\
\text { JO }\end{array}$ & $6.43 \mathrm{E}-01$ & $5.03 \mathrm{E}-06$ & $8.71 E+01$ & $\begin{array}{r}1.30 E+0 \\
3\end{array}$ & $7.68 \mathrm{E}-01$ & $1.39 \mathrm{E}+02$ & $5.05 \mathrm{E}-02$ & $\begin{array}{r}5.21 E+0 \\
0\end{array}$ & $\begin{array}{r}4.04 \\
E+0 \\
3\end{array}$ & $\begin{array}{r}9.50 \mathrm{E}- \\
02\end{array}$ & $1.08 \mathrm{E}-02$ & 3.24E- 05 & $7.50 \mathrm{E}-06$ & 3.35E-01 & 4.96E-06 & $1.02 E+00$ & 1.36E+00 \\
\hline $\begin{array}{l}\text { Autonomous } \\
\text { Fresnel + PV, } \\
\text { MX }\end{array}$ & $1.19 E+00$ & 7.73E-06 & $1.62 \mathrm{E}+02$ & $\begin{array}{r}2.30 E+0 \\
3\end{array}$ & $1.13 E+00$ & $2.02 E+02$ & $1.02 \mathrm{E}-01$ & $\begin{array}{r}2.39 E+0 \\
1\end{array}$ & $\begin{array}{r}7.67 \\
\mathrm{E}+0 \\
3\end{array}$ & $\begin{array}{r}1.68 \mathrm{E}- \\
01\end{array}$ & $1.56 \mathrm{E}-02$ & $4.73 \mathrm{E}-05$ & $1.29 \mathrm{E}-05$ & $5.47 \mathrm{E}-01$ & $8.09 \mathrm{E}-06$ & $1.72 E+00$ & $2.72 E+00$ \\
\hline $\begin{array}{l}\text { Autonomous } \\
\text { HTHP + PV, CL }\end{array}$ & $1.03 E+00$ & $6.01 \mathrm{E}-06$ & $1.48 \mathrm{E}+02$ & $\begin{array}{r}2.05 E+0 \\
3\end{array}$ & $1.29 \mathrm{E}+00$ & $2.03 E+02$ & $1.01 \mathrm{E}-01$ & $\begin{array}{r}1.99 E+0 \\
1\end{array}$ & $\begin{array}{r}6.79 \\
E+0 \\
3\end{array}$ & $\begin{array}{r}1.54 E- \\
01\end{array}$ & $1.75 \mathrm{E}-02$ & $5.21 \mathrm{E}-05$ & $1.15 \mathrm{E}-05$ & $5.15 \mathrm{E}-01$ & $7.86 \mathrm{E}-06$ & $1.60 \mathrm{E}+00$ & $3.04 E+00$ \\
\hline $\begin{array}{l}\text { Autonomous } \\
\text { HTHP + PV, ES }\end{array}$ & $1.50 E+00$ & $6.46 \mathrm{E}-06$ & $1.47 \mathrm{E}+02$ & $\begin{array}{r}2.20 E+0 \\
3\end{array}$ & $1.42 \mathrm{E}+00$ & $2.31 \mathrm{E}+02$ & $1.04 \mathrm{E}-01$ & $\begin{array}{r}1.85 \mathrm{E}+0 \\
1\end{array}$ & $\begin{array}{r}1.04 \\
\mathrm{E}+0 \\
4\end{array}$ & $\begin{array}{r}1.72 E- \\
01\end{array}$ & $2.24 \mathrm{E}-02$ & $5.90 \mathrm{E}-05$ & $1.30 \mathrm{E}-05$ & $5.88 \mathrm{E}-01$ & $8.71 \mathrm{E}-06$ & $1.78 E+00$ & $3.22 E+00$ \\
\hline $\begin{array}{l}\text { Autonomous } \\
\text { HTHP + PV, GR }\end{array}$ & $1.11 \mathrm{E}+00$ & $5.73 \mathrm{E}-06$ & 1.36E +02 & $\begin{array}{r}1.99 E+0 \\
3 \\
\end{array}$ & $1.32 E+00$ & $2.08 E+02$ & $1.29 \mathrm{E}-01$ & $\begin{array}{r}1.16 E+0 \\
1 \\
\end{array}$ & $\begin{array}{r}7.67 \\
E+0 \\
3\end{array}$ & $\begin{array}{r}1.52 E- \\
01 \\
\end{array}$ & $1.88 \mathrm{E}-02$ & $5.31 \mathrm{E}-05$ & $1.16 \mathrm{E}-05$ & $5.03 \mathrm{E}-01$ & 7.42E-06 & $1.50 E+00$ & $2.57 E+00$ \\
\hline $\begin{array}{l}\text { Autonomous } \\
\text { HTHP + PV, JO }\end{array}$ & $9.51 \mathrm{E}-01$ & $4.79 \mathrm{E}-06$ & $1.11 \mathrm{E}+02$ & $\begin{array}{r}1.67 E+0 \\
3\end{array}$ & $1.14 E+00$ & $1.84 \mathrm{E}+02$ & $7.78 \mathrm{E}-02$ & $\begin{array}{r}7.67 E+0 \\
0\end{array}$ & $\begin{array}{r}6.57 \\
E+0 \\
3\end{array}$ & $\begin{array}{r}1.23 E- \\
01\end{array}$ & $1.74 \mathrm{E}-02$ & $4.71 \mathrm{E}-05$ & $9.72 \mathrm{E}-06$ & $4.37 \mathrm{E}-01$ & $6.41 E-06$ & $1.30 E+00$ & $2.11 E+00$ \\
\hline $\begin{array}{c}\text { Autonomous } \\
\text { HTHP + PV, MX }\end{array}$ & $1.87 \mathrm{E}+00$ & $8.17 \mathrm{E}-06$ & $2.06 \mathrm{E}+02$ & $\begin{array}{r}2.95 E+0 \\
3\end{array}$ & $1.63 \mathrm{E}+00$ & $2.73 E+02$ & $1.42 \mathrm{E}-01$ & $\begin{array}{r}2.81 E+0 \\
1\end{array}$ & $\begin{array}{r}1.29 \\
E+0 \\
4\end{array}$ & $\begin{array}{r}2.17 E- \\
01\end{array}$ & $2.56 \mathrm{E}-02$ & $6.85 \mathrm{E}-05$ & $1.68 \mathrm{E}-05$ & $7.19 \mathrm{E}-01$ & $1.09 \mathrm{E}-05$ & $2.20 \mathrm{E}+00$ & $4.16 \mathrm{E}+00$ \\
\hline $\begin{array}{c}\text { HTHP + Grid, } \\
\mathrm{CH}\end{array}$ & $5.64 \mathrm{E}-01$ & $7.21 \mathrm{E}-06$ & $1.86 \mathrm{E}+02$ & $\begin{array}{r}6.55 \mathrm{E}+0 \\
3\end{array}$ & $1.02 E+00$ & $1.58 E+02$ & $1.54 \mathrm{E}-01$ & $\begin{array}{r}2.81 E+0 \\
2\end{array}$ & $\begin{array}{r}3.69 \\
+10 \\
3\end{array}$ & $\begin{array}{r}1.83 E- \\
01\end{array}$ & $3.01 \mathrm{E}-03$ & 4.25E- 05 & $2.70 \mathrm{E}-05$ & $4.72 \mathrm{E}-01$ & $5.89 \mathrm{E}-06$ & $1.89 \mathrm{E}+00$ & $1.28 \mathrm{E}+01$ \\
\hline $\begin{array}{c}\text { HTHP + Grid, } \\
\text { CL }\end{array}$ & 3.77E-01 & $1.00 \mathrm{E}-05$ & $7.43 E+02$ & $\begin{array}{r}1.11 E+0 \\
4\end{array}$ & $6.02 E+00$ & $3.00 \mathrm{E}+02$ & $5.30 \mathrm{E}-01$ & $\begin{array}{r}1.79 E+0 \\
1\end{array}$ & $\begin{array}{r}1.87 \\
E+0 \\
3\end{array}$ & $\begin{array}{r}1.36 E+ \\
00\end{array}$ & $3.50 \mathrm{E}-03$ & 7.41E-05 & 2.60E-05 & $3.59 \mathrm{E}+00$ & 3.67E-05 & $1.41 \mathrm{E}+01$ & $2.48 E+00$ \\
\hline $\begin{array}{c}\text { HTHP + Grid, } \\
\text { ES }\end{array}$ & $6.17 \mathrm{E}-01$ & $8.07 E-06$ & $4.20 \mathrm{E}+02$ & $\begin{array}{r}1.04 E+0 \\
4\end{array}$ & $3.59 E+00$ & $2.17 E+02$ & $1.74 \mathrm{E}-01$ & $\begin{array}{r}2.77 E+0 \\
2\end{array}$ & $\begin{array}{r}3.70 \\
\mathrm{E}+0 \\
3\end{array}$ & $\begin{array}{r}5.63 \mathrm{E}- \\
01\end{array}$ & $3.52 \mathrm{E}-03$ & $6.42 \mathrm{E}-05$ & 4.39E-05 & $1.58 \mathrm{E}+00$ & $1.00 \mathrm{E}-05$ & $5.84 \mathrm{E}+00$ & $4.25 E+00$ \\
\hline $\begin{array}{c}\text { HTHP + Grid, } \\
\text { GR }\end{array}$ & $2.45 \mathrm{E}-01$ & $1.76 \mathrm{E}-05$ & $9.14 E+02$ & $\begin{array}{r}1.51 E+0 \\
4\end{array}$ & $5.79 E+00$ & $5.73 E+02$ & $1.96 \mathrm{E}+00$ & $\begin{array}{r}3.55 E+0 \\
1\end{array}$ & $\begin{array}{r}9.62 \\
\mathrm{E}+0 \\
2\end{array}$ & $\begin{array}{r}8.51 E- \\
01\end{array}$ & $4.21 \mathrm{E}-03$ & $1.24 \mathrm{E}-04$ & $8.08 \mathrm{E}-05$ & $1.63 \mathrm{E}+00$ & $1.77 \mathrm{E}-05$ & $5.05 E+00$ & $5.60 E+00$ \\
\hline
\end{tabular}




\begin{tabular}{|c|c|c|c|c|c|c|c|c|c|c|c|c|c|c|c|c|c|}
\hline & $\begin{array}{l}\text { Land } \\
\text { transform } \\
\text { ation }\end{array}$ & $\begin{array}{c}\text { carcinoge } \\
\text { nic } \\
\text { effects }\end{array}$ & $\begin{array}{c}\text { climate } \\
\text { change } \\
\text { total }\end{array}$ & fossils & $\begin{array}{l}\text { freshwater and } \\
\text { terrestrial } \\
\text { acidification }\end{array}$ & $\begin{array}{l}\text { freshwater } \\
\text { ecotoxicity }\end{array}$ & $\begin{array}{c}\text { freshwater } \\
\text { eutrophicati } \\
\text { on }\end{array}$ & $\begin{array}{c}\text { ionising } \\
\text { radiatio } \\
n\end{array}$ & $\begin{array}{l}\text { land } \\
\text { use }\end{array}$ & $\begin{array}{l}\text { marine } \\
\text { eutrop } \\
\text { hicatio } \\
n\end{array}$ & $\begin{array}{l}\text { minerals } \\
\text { and } \\
\text { metals }\end{array}$ & $\begin{array}{c}\text { non- } \\
\text { carcinogeni } \\
\text { c effects }\end{array}$ & $\begin{array}{c}\text { ozone } \\
\text { layer } \\
\text { depletion }\end{array}$ & $\begin{array}{l}\text { photochemical } \\
\text { ozone creation }\end{array}$ & 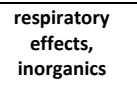 & $\begin{array}{c}\text { terrestrial } \\
\text { eutrophicati } \\
\text { on }\end{array}$ & $\begin{array}{c}\text { water } \\
\text { consump } \\
\text { tion }\end{array}$ \\
\hline Unit & $\mathrm{m}^{2}$ & CTUh & $\begin{array}{l}\mathrm{kg} \mathrm{CO}_{2-} \\
\mathrm{Eq} .\end{array}$ & $\begin{array}{c}\text { megajou } \\
\text { le }\end{array}$ & mol H+-Eq. & стU & kg P-Eq. & $\begin{array}{c}\mathrm{kg} \\
\text { U235- } \\
\text { Eq. }\end{array}$ & $\begin{array}{c}\text { poin } \\
\text { ts }\end{array}$ & $\begin{array}{l}\mathrm{kg} \mathrm{N}- \\
\text { Eq. }\end{array}$ & kg Sb-Eq. & ctuh & kg CFC-11. & kg NMVOC-. & disease $\mathrm{i}$. & mol N-Eq. & $\mathrm{m}^{3}$-eq. \\
\hline HTHP + Grid, IS & $2.60 \mathrm{E}-01$ & $5.91 \mathrm{E}-06$ & $8.26 \mathrm{E}+01$ & $\begin{array}{r}4.52 E+0 \\
2\end{array}$ & $2.84 \mathrm{E}-01$ & $1.19 E+02$ & $2.65 \mathrm{E}-02$ & $\begin{array}{r}1.67 E+0 \\
0\end{array}$ & $\begin{array}{r}2.48 \\
\mathrm{E}+0 \\
2\end{array}$ & $\begin{array}{r}3.84 E- \\
02\end{array}$ & $2.36 \mathrm{E}-03$ & $1.95 \mathrm{E}-05$ & $2.22 \mathrm{E}-06$ & 1.48E-01 & $2.52 E-06$ & 4.32E-01 & $2.68 \mathrm{E}+01$ \\
\hline $\begin{array}{c}\text { HTHP + Grid, } \\
\text { Jo }\end{array}$ & $2.17 E-01$ & $5.95 \mathrm{E}-06$ & $7.22 E+02$ & $\begin{array}{r}1.20 \mathrm{E}+0 \\
4\end{array}$ & $1.77 E+00$ & $2.03 E+02$ & 3.38E-02 & $\begin{array}{r}8.76 \mathrm{E}+0 \\
0\end{array}$ & $\begin{array}{r}6.04 \\
E+0 \\
2\end{array}$ & $\begin{array}{r}3.59 \mathrm{E}- \\
01\end{array}$ & $3.14 \mathrm{E}-03$ & 2.57E-05 & $5.65 E-05$ & $1.13 \mathrm{E}+00$ & 1.12E-05 & $3.81 E+00$ & $1.66 \mathrm{E}+00$ \\
\hline $\begin{array}{c}\text { HTHP + Grid, } \\
\text { MX }\end{array}$ & $2.51 \mathrm{E}-01$ & 9.34E-06 & $8.31 E+02$ & $\begin{array}{r}1.23 E+0 \\
4\end{array}$ & $3.96 \mathrm{E}+00$ & $4.20 E+02$ & 3.93E-01 & $\begin{array}{r}8.93 \mathrm{E}+0 \\
1\end{array}$ & $\begin{array}{r}8.77 \\
E+0 \\
2\end{array}$ & $\begin{array}{r}6.34 E- \\
01\end{array}$ & $3.29 \mathrm{E}-03$ & $5.33 \mathrm{E}-05$ & 6.97E-05 & $1.82 \mathrm{E}+00$ & $1.89 \mathrm{E}-05$ & $6.05 E+00$ & $2.20 E+00$ \\
\hline $\begin{array}{l}\text { HTHP + Grid, } \\
\text { NO }\end{array}$ & $1.50 \mathrm{E}-01$ & $5.33 \mathrm{E}-06$ & 4. $66 \mathrm{E}+01$ & $\begin{array}{r}6.87 E+0 \\
2\end{array}$ & 2.99E-01 & $1.03 E+02$ & $2.98 \mathrm{E}-02$ & $\begin{array}{r}1.47 E+0 \\
1\end{array}$ & $\begin{array}{r}7.04 \\
\mathrm{E}+0 \\
2\end{array}$ & $\begin{array}{r}4.21 E- \\
02\end{array}$ & $2.35 \mathrm{E}-03$ & $1.93 E-05$ & 2.99E-06 & $1.46 \mathrm{E}-01$ & $2.48 \mathrm{E}-06$ & 4.77E-01 & $3.62 E+01$ \\
\hline $\begin{array}{l}\text { Waste heat + } \\
\text { Grid, } \mathrm{CH}\end{array}$ & $3.44 \mathrm{E}-01$ & $5.21 E-06$ & $1.32 E+02$ & $\begin{array}{r}3.94 \mathrm{E}+0 \\
3\end{array}$ & $6.62 \mathrm{E}-01$ & $1.07 E+02$ & $9.94 \mathrm{E}-02$ & $\begin{array}{r}1.58 \mathrm{E}+0 \\
2\end{array}$ & $\begin{array}{r}2.17 \\
\mathrm{E}+0 \\
3\end{array}$ & $\begin{array}{r}1.27 E- \\
01\end{array}$ & $1.83 \mathrm{E}-03$ & $2.70 \mathrm{E}-05$ & $1.64 \mathrm{E}-05$ & 3.35E-01 & 4.21E- 06 & $1.29 \mathrm{E}+00$ & $7.63 E+00$ \\
\hline $\begin{array}{c}\text { Waste heat + } \\
\text { Grid, cL }\end{array}$ & $2.59 \mathrm{E}-01$ & $6.91 \mathrm{E}-06$ & $4.37 E+02$ & $\begin{array}{r}6.45 E+0 \\
3\end{array}$ & $3.40 \mathrm{E}+00$ & $1.89 \mathrm{E}+02$ & $3.06 \mathrm{E}-01$ & $\begin{array}{r}1.57 E+0 \\
1\end{array}$ & $\begin{array}{r}1.20 \\
\mathrm{E}+0 \\
3\end{array}$ & $\begin{array}{r}\text { 7.69E- } \\
01\end{array}$ & 2.13E-03 & 4.53E- 05 & $1.62 \mathrm{E}-05$ & $2.04 E+00$ & 2.12E-05 & $7.98 \mathrm{E}+00$ & $1.88 \mathrm{E}+00$ \\
\hline $\begin{array}{l}\text { Waste heat + } \\
\text { Grid, ES }\end{array}$ & $3.55 \mathrm{E}-01$ & $5.15 \mathrm{E}-06$ & $2.45 E+02$ & $\begin{array}{r}5.90 E+0 \\
3\end{array}$ & $2.03 E+00$ & $1.30 \mathrm{E}+02$ & $1.00 \mathrm{E}-01$ & $\begin{array}{r}1.55 \mathrm{E}+0 \\
2\end{array}$ & $\begin{array}{r}2.10 \\
\mathrm{E}+0 \\
3\end{array}$ & $\begin{array}{r}3.21 E- \\
01\end{array}$ & 2.04E-03 & $3.68 \mathrm{E}-05$ & 2.50E-05 & $9.10 \mathrm{E}-01$ & $6.05 \mathrm{E}-06$ & $3.33 E+00$ & $2.48 \mathrm{E}+00$ \\
\hline $\begin{array}{c}\text { Waste heat + } \\
\text { Grid, GR }\end{array}$ & $1.51 \mathrm{E}-01$ & $1.07 \mathrm{E}-05$ & $5.20 E+02$ & $\begin{array}{r}8.56 \mathrm{E}+0 \\
3\end{array}$ & $3.26 \mathrm{E}+00$ & $3.34 E+02$ & $1.09 E+00$ & $\begin{array}{r}2.11 \mathrm{E}+0 \\
1\end{array}$ & $\begin{array}{r}6.07 \\
E+0 \\
2\end{array}$ & $\begin{array}{r}4.88 E- \\
01\end{array}$ & $2.46 \mathrm{E}-03$ & 7.13E-05 & 4.56E-05 & $9.53 \mathrm{E}-01$ & $1.04 \mathrm{E}-05$ & $2.96 \mathrm{E}+00$ & $3.23 E+00$ \\
\hline $\begin{array}{l}\text { Waste heat + } \\
\text { Grid, Is }\end{array}$ & $1.53 \mathrm{E}-01$ & $4.09 E-06$ & $5.56 \mathrm{E}+01$ & $\begin{array}{r}3.83 E+0 \\
2\end{array}$ & $2.00 E-01$ & $8.04 E+01$ & $2.19 \mathrm{E}-02$ & $\begin{array}{r}1.23 \mathrm{E}+0 \\
0\end{array}$ & $\begin{array}{r}1.85 \\
\mathrm{E}+0 \\
2\end{array}$ & $\begin{array}{r}3.61 E- \\
02\end{array}$ & $1.41 \mathrm{E}-03$ & $1.32 E-05$ & $1.90 \mathrm{E}-06$ & $1.25 \mathrm{E}-01$ & $1.90 \mathrm{E}-06$ & $3.75 E-01$ & $1.49 E+01$ \\
\hline $\begin{array}{c}\text { Waste heat + } \\
\text { Grid, Jo }\end{array}$ & $1.29 \mathrm{E}-01$ & 4.11E-06 & $4.09 E+02$ & $\begin{array}{r}6.79 E+0 \\
3\end{array}$ & $1.02 E+00$ & $1.27 E+02$ & $2.59 \mathrm{E}-02$ & $\begin{array}{r}5.14 \mathrm{E}+0 \\
0\end{array}$ & $\begin{array}{r}3.82 \\
E+0 \\
2\end{array}$ & $\begin{array}{r}2.13 E- \\
01\end{array}$ & $1.84 \mathrm{E}-03$ & $1.67 E-05$ & $3.19 \mathrm{E}-05$ & $6.68 \mathrm{E}-01$ & $6.70 \mathrm{E}-06$ & $2.24 E+00$ & $1.00 \mathrm{E}+00$ \\
\hline $\begin{array}{c}\text { Waste heat + } \\
\text { Grid, } M X\end{array}$ & $1.79 \mathrm{E}-01$ & $6.55 \mathrm{E}-06$ & $4.89 \mathrm{E}+02$ & $\begin{array}{r}7.17 \mathrm{E}+0 \\
3\end{array}$ & $2.28 \mathrm{E}+00$ & $2.55 E+02$ & $2.37 \mathrm{E}-01$ & $\begin{array}{r}5.63 \mathrm{E}+0 \\
1\end{array}$ & $\begin{array}{r}6.75 \\
\mathrm{E}+0 \\
2\end{array}$ & $\begin{array}{r}3.78 E- \\
01\end{array}$ & $2.02 E-03$ & $3.40 \mathrm{E}-05$ & 4.01E-05 & $1.08 \mathrm{E}+00$ & $1.14 \mathrm{E}-05$ & $3.60 E+00$ & 1.53E+00 \\
\hline $\begin{array}{c}\text { Waste heat + } \\
\text { Grid, NO }\end{array}$ & $8.97 E-02$ & $3.48 E-06$ & $3.37 E+01$ & $\begin{array}{r}4.96 E+0 \\
2\end{array}$ & $1.92 \mathrm{E}-01$ & $6.45 E+01$ & $1.78 E-02$ & $\begin{array}{r}8.38 \mathrm{E}+0 \\
0\end{array}$ & $\begin{array}{r}4.23 \\
\mathrm{E}+0 \\
2\end{array}$ & $\begin{array}{r}2.99 \mathrm{E}- \\
02\end{array}$ & 1.37E-03 & $1.15 \mathrm{E}-05$ & $2.13 \mathrm{E}-06$ & $1.07 E-01$ & $1.73 \mathrm{E}-06$ & $3.31 E-01$ & $2.01 E+01$ \\
\hline $\begin{array}{c}\text { Waste heat + } \\
\mathrm{PV}+\text { Battery, } \\
\mathrm{CL}\end{array}$ & $6.16 \mathrm{E}-01$ & $4.72 E-06$ & $1.14 E+02$ & $\begin{array}{r}1.53 E+0 \\
3\end{array}$ & $8.32 \mathrm{E}-01$ & $1.36 \mathrm{E}+02$ & 7.35E-02 & $\begin{array}{r}1.68 \mathrm{E}+0 \\
1\end{array}$ & $\begin{array}{r}3.88 \\
\mathrm{E}+0 \\
3\end{array}$ & $\begin{array}{r}1.16 E- \\
01\end{array}$ & $9.72 \mathrm{E}-03$ & 3.33E-05 & $8.30 \mathrm{E}-06$ & $3.70 \mathrm{E}-01$ & $5.56 \mathrm{E}-06$ & $1.18 \mathrm{E}+00$ & $2.18 \mathrm{E}+00$ \\
\hline $\begin{array}{c}\text { Waste heat + } \\
\text { PV + Battery, } \\
\text { ES }\end{array}$ & 8.33E-01 & $4.27 E-06$ & $9.71 E+01$ & $\begin{array}{r}1.47 \mathrm{E}+0 \\
3\end{array}$ & $8.47 E-01$ & $1.38 \mathrm{E}+02$ & $6.18 \mathrm{E}-02$ & $\begin{array}{r}1.42 E+0 \\
1\end{array}$ & $\begin{array}{r}5.73 \\
\mathrm{E}+0 \\
3\end{array}$ & $\begin{array}{r}1.09 E- \\
01\end{array}$ & $1.23 \mathrm{E}-02$ & $3.40 \mathrm{E}-05$ & $8.22 \mathrm{E}-06$ & $3.70 \mathrm{E}-01$ & $5.33 \mathrm{E}-06$ & $1.12 E+00$ & $1.92 \mathrm{E}+00$ \\
\hline $\begin{array}{l}\text { Waste heat + } \\
\text { PV + Battery, } \\
\text { GR }\end{array}$ & $6.23 \mathrm{E}-01$ & $4.26 \mathrm{E}-06$ & $9.77 E+01$ & $\begin{array}{r}1.42 E+0 \\
3\end{array}$ & $8.31 \mathrm{E}-01$ & $1.36 \mathrm{E}+02$ & $9.82 E-02$ & $\begin{array}{r}8.17 E+0 \\
0\end{array}$ & $\begin{array}{r}4.25 \\
\mathrm{E}+0 \\
3\end{array}$ & $\begin{array}{r}1.09 E- \\
01\end{array}$ & $1.04 \mathrm{E}-02$ & $3.30 \mathrm{E}-05$ & $8.04 \mathrm{E}-06$ & $3.41 \mathrm{E}-01$ & $4.84 \mathrm{E}-06$ & $1.04 E+00$ & $1.59 \mathrm{E}+00$ \\
\hline $\begin{array}{c}\text { Waste heat + } \\
\text { PV + Battery, } \\
\text { Jo }\end{array}$ & $5.28 \mathrm{E}-01$ & $3.49 \mathrm{E}-06$ & $7.74 E+01$ & $\begin{array}{r}1.16 \mathrm{E}+0 \\
3\end{array}$ & $6.81 \mathrm{E}-01$ & $1.16 \mathrm{E}+02$ & $4.97 E-02$ & $\begin{array}{r}4.55 \mathrm{E}+0 \\
0\end{array}$ & $\begin{array}{r}3.62 \\
\mathrm{E}+0 \\
3\end{array}$ & $\begin{array}{r}8.52 \mathrm{E}- \\
02\end{array}$ & $9.57 \mathrm{E}-03$ & $2.83 \mathrm{E}-05$ & $6.49 \mathrm{E}-06$ & 2.91E-01 & $4.10 \mathrm{E}-06$ & $8.77 \mathrm{E}-01$ & $1.25 E+00$ \\
\hline $\begin{array}{l}\text { Waste heat + } \\
\text { PV + Battery, } \\
\text { MX }\end{array}$ & $1.06 \mathrm{E}+00$ & $5.92 \mathrm{E}-06$ & $1.50 E+02$ & $\begin{array}{r}2.11 E+0 \\
3\end{array}$ & $1.02 E+00$ & $1.75 E+02$ & $1.01 \mathrm{E}-01$ & $\begin{array}{r}2.30 \mathrm{E}+0 \\
1\end{array}$ & $\begin{array}{r}7.18 \\
E+0 \\
3\end{array}$ & $\begin{array}{r}1.51 \mathrm{E}- \\
01\end{array}$ & $1.41 \mathrm{E}-02$ & 4.23E-05 & $1.14 E-05$ & 4.83E-01 & 7.06E-06 & $1.51 \mathrm{E}+00$ & $2.60 E+00$ \\
\hline
\end{tabular}




\section{Visualization in spider graphs}

Spider graphs for all DACCS system configurations on all environmental impact categories are presented in Figure D. The environmental impacts per category are normalized to the maximum score - on an environmental impact category - of the considered DACCS configurations. The maximum scores of environmental impact categories can be found in Red in Table 8 .

Figure D. Spider graphs illustrating environmental trade-offs of DACCS systems configurations on all environmental impact categories. CE $=$ Carcinogenic Effects, CC = Climate Change Total, FO = Fossils, FTA = Freshwater And Terrestrial Acidification, FET = Freshwater Ecotoxicity, $F E=$ Freshwater Eutrophication, $I R=$ Ionising Radiation, $L T=$ Land Transformation, $L U=$ Land Use, ME = Marine Eutrophication, MM = Minerals And Metals, NCE = Non-Carcinogenic Effects, OZD = Ozone Layer Depletion, $P O C=$ Photochemical Ozone Creation, REI = Respiratory Effects; Inorganics, TE = Terrestrial Eutrophication, WC = Water Consumption.
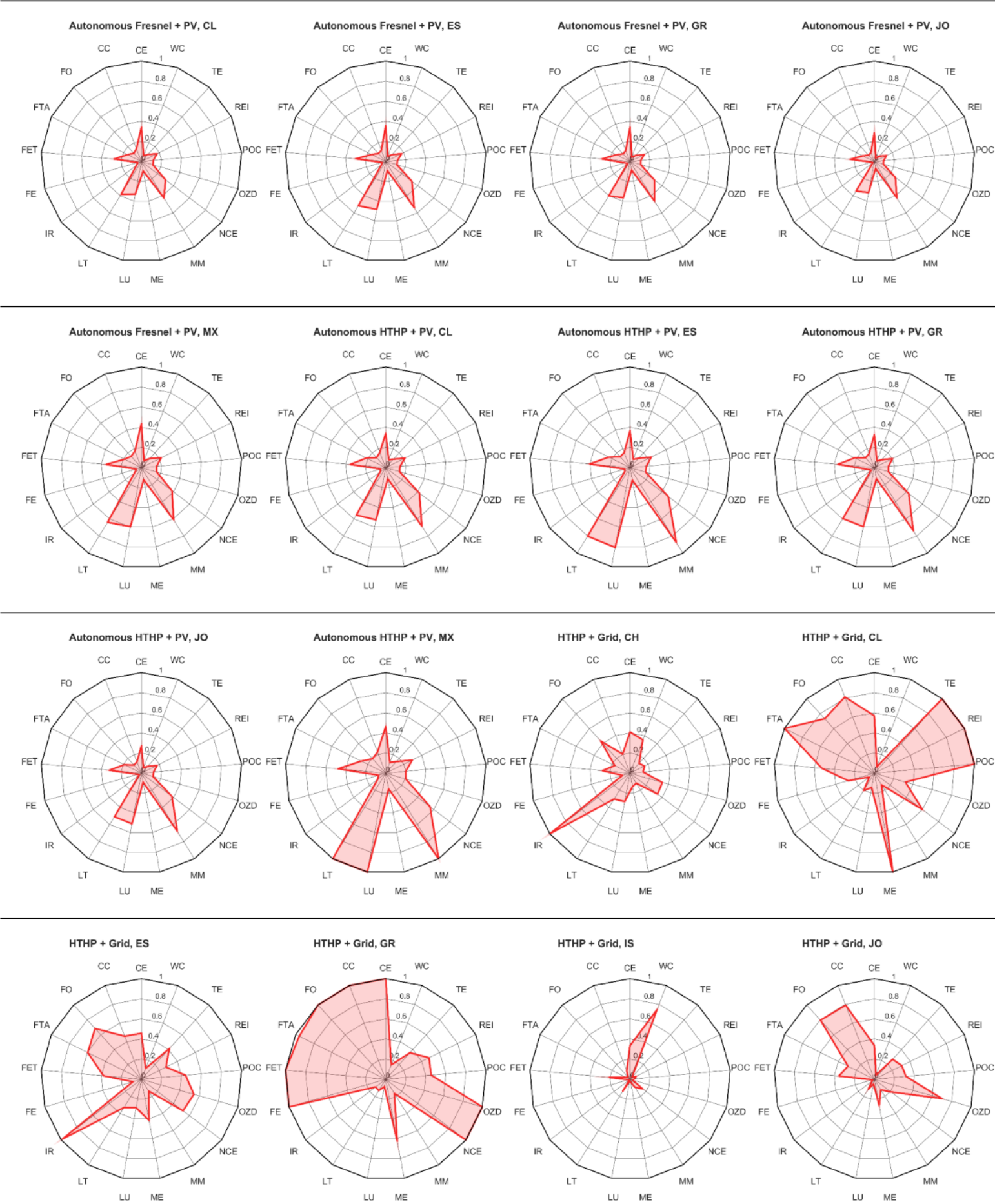

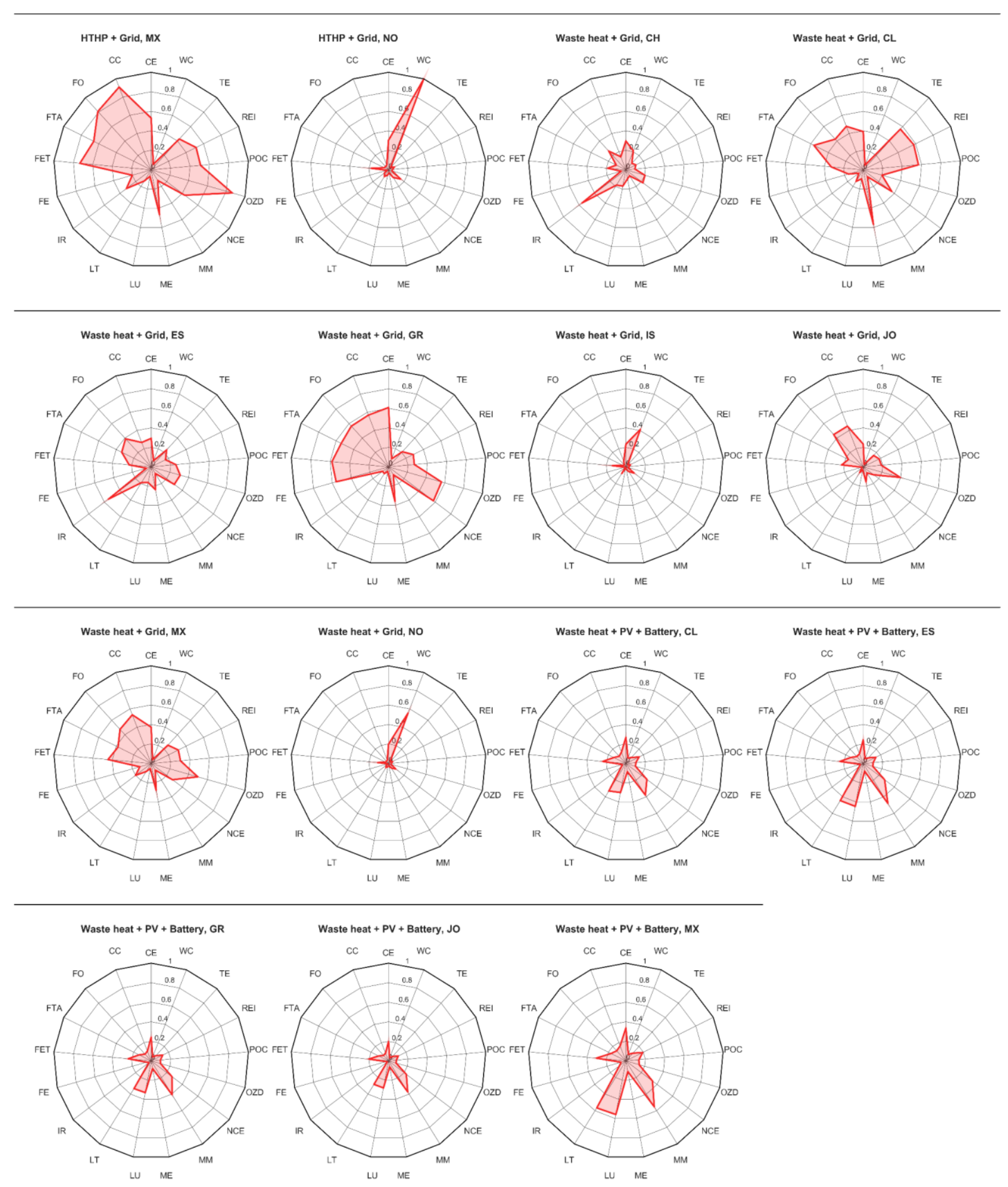


\section{S7. LCA results for land transformation}

Figure E shows the results on land transformation for each DACCS configuration considered in the main analysis. We aggregate all life cycle inventory flows containing "Transformation, from.." within this analysis in order to represent land use. The stacked bars labeled with "direct" represent direct land use at the DAC site: area occupied by the DAC unit itself, by solar PV and solar heat installations, and by energy storage units, while the stacked bars labeled with "life-cycle" represent overall land use including indirect contributions.

Autonomous energy systems exhibit large land transformation, mainly due to the installation of PV panels (on the ground) and the fresnel heat collector with large surface area requirements, while the DAC unit as separate system has very low land transformation. In reality, direct land transformation is also generated from the installation of pipelines, but are excluded in the direct land transformation impacts of Figure E, since land is not directly transformed at the DAC location. Therefore, the main indirect contribution originates from land use of pipelines, and scales with $\mathrm{CO}_{2}$ transportation distance.

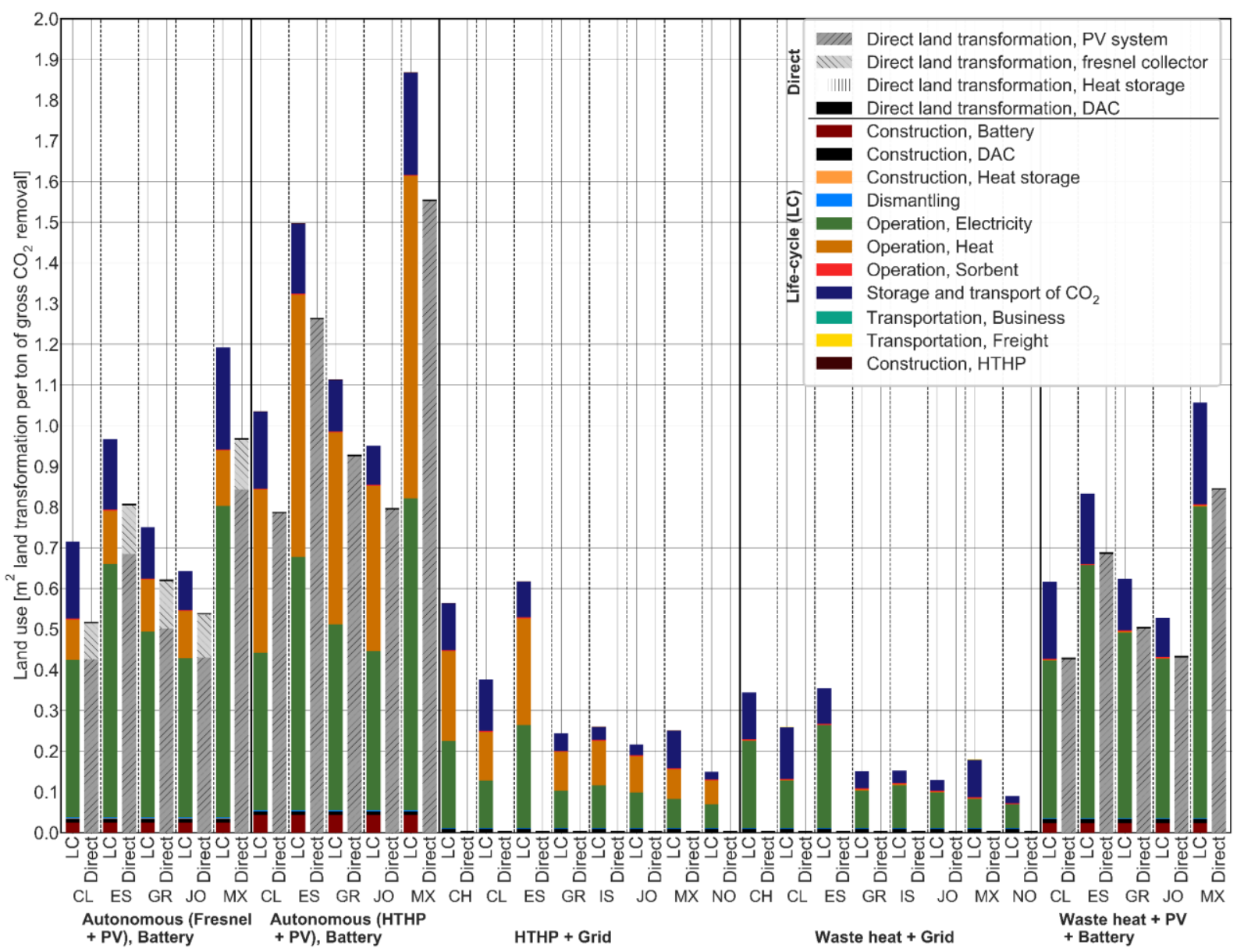

Figure E. Land transformation results for all considered DACCS configurations and countries of our main analysis. For each country, the total life-cycle land transformation (including indirect land transformation) is represented by the colored stacked bars. The direct land use transformation at the DAC site for the main system components is represented by the black-grey-white colored bars: PV system, fresnel collector, heat storage and the DAC unit. $L C=L i f e-C y c l e, C L=C h i l e, E S=$ Spain, $G R=$ Greece, $J O=$ Jordan, $M X=$ Mexico, $C H=S w i t z e r l a n d, I S=$ Iceland and NO = Norway. 


\section{S8. Sensitivity analysis: Reduced electricity consumption for $\mathrm{CO}_{2}$ capture}

Figure $\mathrm{F}$ shows the sensitivity analysis regarding electricity consumption of the DAC unit and the effects on life-cycle GHG emissions per ton of $\mathrm{CO}_{2}$ captured.

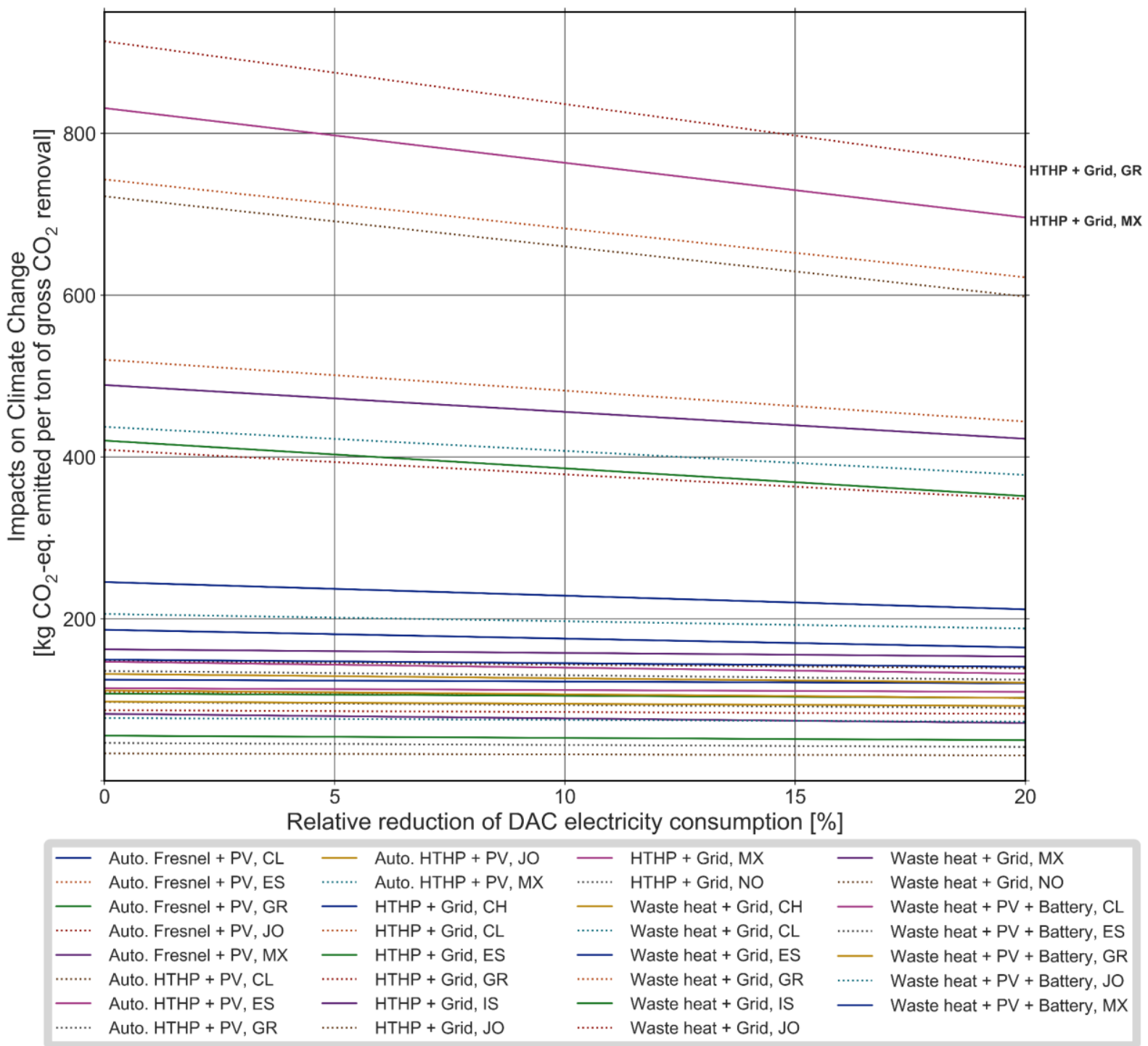

Figure F. Sensitivity analysis on the Climate Change impact category with a reduction of electricity consumption for $\mathrm{CO}_{2}$ capture. 


\section{S9. Ecoinvent 3.6 datasets used for Figure 3.}

Table 9 shows ecoinvent datasets used - represented on the x-axis - in Figure 3 of the main article.

Table 9. Ecoinvent 3.6 datasets used on the x-axis - representing the Climate Change impacts of electricity datasets - in Figure 3 of the main article.

\begin{tabular}{|l|l|}
\hline $\begin{array}{l}\text { Electricity dataset } \\
\text { in Figure 3 }\end{array}$ & ecoinvent reference product | name | location | unit | database \\
\hline Wind & $\begin{array}{l}\text { electricity, high voltage | electricity production, wind, >3MW turbine, onshore | RoW | kilowatt hour | cutoff 36 [50\%] } \\
\text { electricity, high voltage | electricity production, wind, 1-3MW turbine, offshore | RoW | kilowatt hour | cutoff 36 [50\%] }\end{array}$ \\
\hline Iceland & electricity, low voltage | market for electricity, low voltage | IS | kilowatt hour | cutoff 36 \\
\hline Photovoltaics & $\begin{array}{l}\text { electricity, low voltage | electricity production, photovoltaic, 3kWp slanted-roof installation, multi-Si, panel, mounted | ES } \\
\text { | kilowatt hour | cutoff 36 }\end{array}$ \\
\hline Switzerland & electricity, low voltage | market for electricity, low voltage | CH | kilowatt hour | cutoff 36 \\
\hline Denmark & electricity, low voltage | market for electricity, low voltage | DK | kilowatt hour | cutoff 36 \\
\hline United Kingdom & electricity, low voltage | market for electricity, low voltage | GB | kilowatt hour | cutoff 36 \\
\hline Europe [ENTSO-E] & electricity, low voltage | market group for electricity, low voltage | ENTSO-E | kilowatt hour | cutoff 36 \\
\hline Natural gas & electricity, high voltage | electricity production, natural gas, combined cycle power plant | RoW | kilowatt hour | cutoff 36 \\
\hline Germany & electricity, low voltage | market for electricity, low voltage | DE | kilowatt hour | cutoff 36 \\
\hline World & electricity, low voltage | market group for electricity, low voltage | GLO | kilowatt hour | cutoff 36 \\
\hline Oil & electricity, high voltage | electricity production, oil | RoW | kilowatt hour | cutoff 36 \\
\hline
\end{tabular}




\section{References}

Alcalde, J. et al. (2018) 'Estimating geological CO2 storage security to deliver on climate mitigation', Nature Communications. doi: $10.1038 / \mathrm{s} 41467-018-04423-1$.

Anthonsen, K. L. et al. (2013) 'CO2 storage potential in the Nordic region', in Energy Procedia. doi: 10.1016/j.egypro.2013.06.421.

Beuttler, C., Charles, L. and Wurzbacher, J. (2019) 'The Role of Direct Air Capture in Mitigation of Anthropogenic Greenhouse Gas Emissions', Frontiers in Climate. doi: 10.3389/fclim.2019.00010.

Deutz, S. and Bardow, A. (2021) 'Life-cycle assessment of an industrial direct air capture process based on temperature-vacuum swing adsorption', Nature Energy, 6(2), pp. 203-213. doi: 10.1038/s41560-020-00771-9.

Ecoinvent (2020) ecoinvent 3.6. Available at: https://www.ecoinvent.org/database/older-versions/ecoinvent-36/ecoinvent-36.html (Accessed: 30 November 2020).

ESMAP et al. (2020) Global Solar Atlas, Global Solar Atlas. Available at: https://globalsolaratlas.info/map.

Fasihi, M., Efimova, O. and Breyer, C. (2019) 'Techno-economic assessment of CO 2 direct air capture plants', Journal of Cleaner Production . doi: 10.1016/j.jclepro.2019.03.086.

van der Giesen, C. et al. (2017) ‘ A Life Cycle Assessment Case Study of Coal-Fired Electricity Generation with Humidity Swing Direct Air Capture of CO 2 versus MEA-Based Postcombustion Capture ', Environmental Science \& Technology. doi: 10.1021/acs.est.6b05028.

Global CCS Institute (2011) The global status of CCS: 2010 . Available https://www.globalccsinstitute.com/archive/hub/publications/12776/global-status-ccs-2010.pdf.

Hanna, R. et al. (2021) 'Emergency deployment of direct air capture as a response to the climate crisis', Nature Communications. doi: $10.1038 / \mathrm{s} 41467-020-20437-0$.

Hendriks, Chris; Wina, Graus; and Bergen V., F. (2004) Global Carbon Dioxide Storage Potential, Report.

Holloway, S. et al. (2006) 'Chapter 5: Carbon Dioxide Transport, Injection and Geological Storage', 2006 IPCC Guidelines for National Greenhouse Gas Inventories.

Industrial Solar (2021) Fresnel Collector LF-11. Available at: https://www.industrial-solar.de/technologies/fresnel-collector/ (Accessed: 7 January 2021)

de Jonge, M. M. J. et al. (2019) 'Life cycle carbon efficiency of Direct Air Capture systems with strong hydroxide sorbents', International Journal of Greenhouse Gas Control. doi: 10.1016/j.ijggc.2018.11.011.

Kelemen, P. et al. (2019) 'An Overview of the Status and Challenges of CO2 Storage in Minerals and Geological Formations', Frontiers in Climate doi: 10.3389/fclim.2019.00009.

Kurup, P. et al. (2019) 'Initial Thermal Energy Yield Potential for the Use of Concentrating Solar Power (CSP) for Coal Hybridization in India', NREL/TP- 6A20-74024.

Schmidt, T. S. et al. (2019) 'Additional Emissions and Cost from Storing Electricity in Stationary Battery Systems', Environmental Science and Technology. doi: 10.1021/acs.est.8b05313.

Solargis (2020) Download solar resource maps and GIS data for 200+ countries, (C) 2020 The World Bank, Source: Global Solar Atlas 2.0, Solar resource data: Solargis. Available at: https://solargis.com/maps-and-gis-data/download/world (Accessed: 30 November 2020).

Viebahn, P., Scholz, A. and Zelt, O. (2019) 'The potential role of direct air capture in the German energy research program-results of a multidimensional analysis', Energies. doi: 10.3390/en12183443.

Volkart, K., Bauer, C. and Boulet, C. (2013) 'Life cycle assessment of carbon capture and storage in power generation and industry in Europe', International Journal of Greenhouse Gas Control. doi: 10.1016/j.ijggc.2013.03.003.

Volkswagen (2006) Golf dimensions - 5-door Exterior dimensions Golf dimensions - 5-door Interior dimensions. Available at: https://www.volkswagen.co.uk/files/live/sites/vwuk/files/pdf/Brochures/golf-dimensions.pdf.

Wildbolz, C. (2007) Life Cycle Assessment of Selected Technologies for CO 2 Transport and Sequestration, Environmental Engineering. ETH Zurich. 
SI_Life Cycle Assessment of Direct Air Carbon Capture an... (4.03 MiB) view on ChemRxiv • download file 


\title{
Life Cycle Assessment of Direct Air Carbon Capture and Storage with Low-Carbon Energy Sources
}

\author{
Tom Terlouw, ${ }^{*,+, \pm \uparrow}$ Karin Treyer, ${ }^{\dagger}$ Christian Bauer, ${ }^{\dagger}$ and Marco Mazzotti ${ }^{\ddagger}$ \\ $\dagger$ Paul Scherrer Institut, Laboratory for Energy Systems Analysis, Paul Scherrer Institut, \\ 5232 Villigen PSI, Switzerland \\ $\ddagger$ Institute of Energy and Process Engineering, ETH Zürich, Zürich 8092, Switzerland \\ ฯCorresponding author \\ E-mail: tom.terlouw@psi.ch
}

\begin{abstract}
Direct air carbon capture and storage (DACCS) is an emerging carbon dioxide removal technology, which has the potential to remove large amounts of $\mathrm{CO}_{2}$ from the atmosphere. However, DACCS systems have hardly been evaluated regarding their environmental life-cycle performance. We present a comprehensive life cycle assessment (LCA) of different DACCS systems with low-carbon electricity and heat sources required for the $\mathrm{CO}_{2}$ capture process - both stand-alone and grid-connected system configurations. The results demonstrate negative greenhouse gas (GHG) emissions i.e. a net removal of $\mathrm{CO}_{2}$ from the atmosphere - for all eight selected locations and five system layouts. Highest GHG removal potential is found for Norway with a gridcoupled system layout and waste heat usage with a GHG removal efficiency of almost 97\%. Autonomous system layouts - entirely supplied by solar energy - prove to be a promising alternative at locations with high solar irradiation to avoid the consumption
\end{abstract}


of fossil fuel based grid electricity and heat: Their GHG removal efficiency (79\%-91\%) is at a similar - or even higher - level as grid-connected DAC in countries with rather lowcarbon electricity supply and available waste heat, such as Switzerland with a GHG removal efficiency of $87 \%$. The analysis of environmental burdens other than GHG emissions shows some trade-offs associated with $\mathrm{CO}_{2}$ removal - especially land transformation for system layouts with PV electricity supply - and confirms the need for a comprehensive LCA approach. Further, the sensitivity analysis reveals the importance of selecting appropriate locations for grid-coupled all-electric system layouts, since the deployment of DACCS at geographic locations with $\mathrm{CO}_{2}$-intensive grid electricity mixes leads to net GHG emissions instead of GHG removal today. However, a prospective global analysis with the integration of two future energy scenarios shows net-negative GHG emissions for the all-electric system layouts on all continents in 2040.

\section{Keywords}

Life cycle assessment (LCA), Direct air carbon capture and storage (DACCS), Carbon dioxide removal (CDR), Negative emission technologies (NETs).

\section{Synopsis}

We provide a comprehensive life cycle assessment of different direct air carbon capture and storage configurations to evaluate the environmental performance of this supposedly decisive technology in future low-carbon energy systems.

\section{Introduction}

Carbon dioxide removal (CDR) technologies, or negative emission technologies (NETs), are expected to play a crucial role in the decarbonization of the global energy system, with

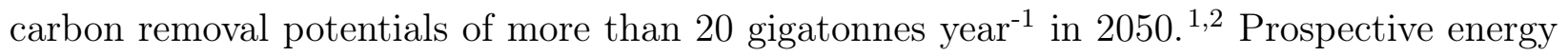


scenarios, generated by integrated assessment models (IAMs), demonstrate that the $1.5^{\circ} \mathrm{C}$ target of the Paris Agreement is likely to be infeasible without the large-scale deployment of CDR technologies, and most IAM scenarios rely on the large-scale deployment of CDR technologies to have a chance of more than $50 \%$ to reach the $2.0^{\circ} \mathrm{C}$ target. ${ }^{3,4}$

Further, CDR technologies are required to a greater extent when climate mitigation is postponed, to compensate for an overshoot of GHG emissions. ${ }^{5}$ A wide portfolio of CDR technologies has been proposed, such as the application of biochar, enhanced weathering (EW), ocean fertilisation (OF), bioenergy with carbon capture and storage (BECCS) and direct air carbon capture and storage (DACCS). ${ }^{1,2,6}$ However, CDR technologies can have substantial environmental side-effects, such as impacts on land, water and/or soil. ${ }^{4,6}$ For example, CDR technologies associated with biomass feedstock (e.g. BECCS and biochar) typically result in intensive land use, soil quality changes and water consumption, while potential unintended side-effects of other CDR technologies (e.g. OF) still need to be investigated. ${ }^{4,6,7}$ DACCS systems could largely avoid impacts on the water and food security nexus and can be considered as technology ready for small-scale deployment. ${ }^{4,6,8,9}$ DACCS systems aim to extract $\mathrm{CO}_{2}$ from ambient air and permanently store the captured $\mathrm{CO}_{2}$ in a geological storage medium. ${ }^{6}$

Direct air capture (DAC) usually includes two steps, the adsorption (or absorption) step and the desorption (regeneration) step. During the former process, sorbents with strong absorption characteristics are used in a contacting area to bind $\mathrm{CO}_{2}$, which is challenging due the extreme dilute concentration of $\mathrm{CO}_{2}$ in ambient air. ${ }^{8}$ The regeneration process aims to regenerate the sorbents and to separate $\mathrm{CO}_{2}$. The latter process is energy-intensive due to the requirement of heat at relatively high temperature levels. ${ }^{8}$

DACCS systems offer some flexibility compared to other CDR technologies, since they can remove $\mathrm{CO}_{2}$ independently from point sources, both in time and space. ${ }^{6,10}$ Consequently, optimal locations can be selected, considering $\mathrm{CO}_{2}$ storage potential and local costs for energy supply. ${ }^{11}$ DAC systems are usually based either on aqueous solutions with high temperature 
regeneration (HT DAC), or on solid sorbents with low temperature regeneration (LT DAC). ${ }^{8}$ This distinction is established based on the temperature level and the sorbent used in the $\mathrm{CO}_{2}$ capture process. ${ }^{8}$ Few companies currently offer such DAC systems. Carbon Engineering (Canada) implemented HT DAC systems on the North-American market. ${ }^{8,12}$ Climeworks (Switzerland) installed pilot LT DAC plants in Europe and realized the first DACCS project with negative $\mathrm{CO}_{2}$ emissions in Hellisheiði (Iceland) in 2017. ${ }^{8,13}$ Global Thermostat recently deployed pilot and demonstration plants based on LT DAC in the United States. ${ }^{8}$ Other DACCS initiatives and pilot plants usually apply the LT DAC approach. ${ }^{14}$ Generally, not all current DACCS demonstration or pilot plants are designed as DACCS - i.e. with permanent storage of captured $\mathrm{CO}_{2}$ - but rather connect to an industrial carbon utilisation process to reach carbon neutrality.

As emerging technology with a supposedly decisive role in future low-carbon energy systems, DACCS systems must be thoroughly evaluated regarding their environmental performance in a transparent and consistent way over their entire lifetime ${ }^{6}$ Life cycle assessment (LCA) is a suitable and flexible assessment tool to identify environmental hotspots (i.e. the main contributors to environmental burdens) and to evaluate the total life-cycle environmental performance of a product or service. ${ }^{15,16}$ Only few DACCS LCA studies - with mostly limited scopes - have been conducted so far.

De Jonge et al. ${ }^{17}$ assessed the life-cycle carbon removal efficiency of a HT DAC system and determined the main environmental contributors to overall LCA scores. The contribution analysis revealed a high environmental impact due to energy needed for the $\mathrm{CO}_{2}$ capture process. A recent DACCS LCA study conducted by Deutz and Bardow ${ }^{18}$ showed that two commercial LT DAC(CS) plants - in Hinwil (Switzerland) and Hellisheiði (Iceland) produced and operated by Climeworks - achieved GHG removal efficiencies of $85 \%$ and $93 \%$, respectively. The latter study also determined the environmental impacts of six different adsorbents and concluded that climate benefits are mainly influenced by energy sources used for $\mathrm{CO}_{2}$ capture. 
To the best of our knowledge, no comprehensive LCA of DACCS has been published in the scientific literature. We acknowledge that the study of Deutz and Bardow ${ }^{18}$ is comprehensive for the DAC system and the associated supply chains, but lacks a detailed assessment of the $\mathrm{CO}_{2}$ storage stage, since their work only considered electricity demand for $\mathrm{CO}_{2}$ injection. However, the $\mathrm{CO}_{2}$ storage stage includes for example environmental impacts from infrastructure (e.g. pipelines, injection wells and the compression station), the drilling of injection wells and $\mathrm{CO}_{2}$ leakage during the transportation of $\mathrm{CO}_{2} \cdot{ }^{19,20}$ Besides, the latter study excludes energy storage when intermittent (renewable) energy sources are integrated and claim that their energy system layouts can be installed at remote locations, while we believe that appropriate energy storage units need to be considered when (renewable) intermittent energy sources are used for the $\mathrm{CO}_{2}$ capture process - which inevitably leads to additional environmental impacts. Further, the DACCS study of de Jonge et al. ${ }^{17}$ only focused on the life cycle carbon efficiency, hence excluded other potentially important environmental impacts. In addition, the latter study reported limitations regarding the quality of their life cycle inventory (LCI) data for the DAC infrastructure.

Other available LCA studies mainly focus on DAC, thereby excluding the carbon storage stage required for permanent $\mathrm{CO}_{2}$ removal from the atmosphere. In addition, these LCAs are simplified regarding LCA modelling choices, such as the exclusion of life-cycle phases and of environmental impact categories besides climate change. ${ }^{6}$ Further, some studies do not consider a certain amount of $\mathrm{CO}_{2}$ equivalents removed from the atmosphere as functional unit, which impedes the comparison of different CDR technologies. ${ }^{6}$ Essentially, a comprehensive LCA on the entire DACCS supply chain, which assesses multiple environmental impact categories, uses an appropriate functional unit, is transparent in the methodology used, thoroughly assesses all life-cycle stages and examines a wide set of energy sources for $\mathrm{CO}_{2}$ capture including autonomous system designs, is missing.

In this context, the contributions of this paper can be summarized as follows:

- We present a detailed and transparent LCA of a LT DACCS system - based on Clime- 
works' technology and verified with available data on LT DAC - with different electricity (i.e. grid and photovoltaics (PV) power) and heat sources (i.e. electricity, waste and solar heat) for $\mathrm{CO}_{2}$ capture.

- Different innovative and autonomous system layouts are included, namely the integration of high temperature heat pumps (HTHPs), the integration of a fresnel solar heat plant at locations with high solar irradiation and system layouts with electricity and heat storage.

- We include several processes needed for $\mathrm{CO}_{2}$ storage - parameterized on transportation distance, geographical storage location and storage depth: energy needs for compression, infrastructure requirements (e.g. pipelines and compression stations), drilling of boreholes, country-specific electricity for the injection of $\mathrm{CO}_{2}$, and $\mathrm{CO}_{2}$ leakage resulting from the transportation in pipelines.

- We address land transformation in detail: while grid-connected DACCS systems could exhibit low land requirements compared to other CDR technologies, ${ }^{6}$ direct solar electricity and heat supply for the DAC unit might result in substantial land transformation. ${ }^{4,14}$

- A global analysis is included for all-electric grid connected DACCS systems.

- We integrate two future IAM scenarios ${ }^{21-23}$ and modify the ecoinvent background LCA database to determine the future global carbon removal potential of grid connected DACCS systems.

The structure of the paper is as follows. Section 2 describes the methodology, where LCA modelling steps and different DACCS system layouts are discussed. Section 3 presents the LCA results, discussions and limitations. Finally, conclusions are drawn in Section 4. 


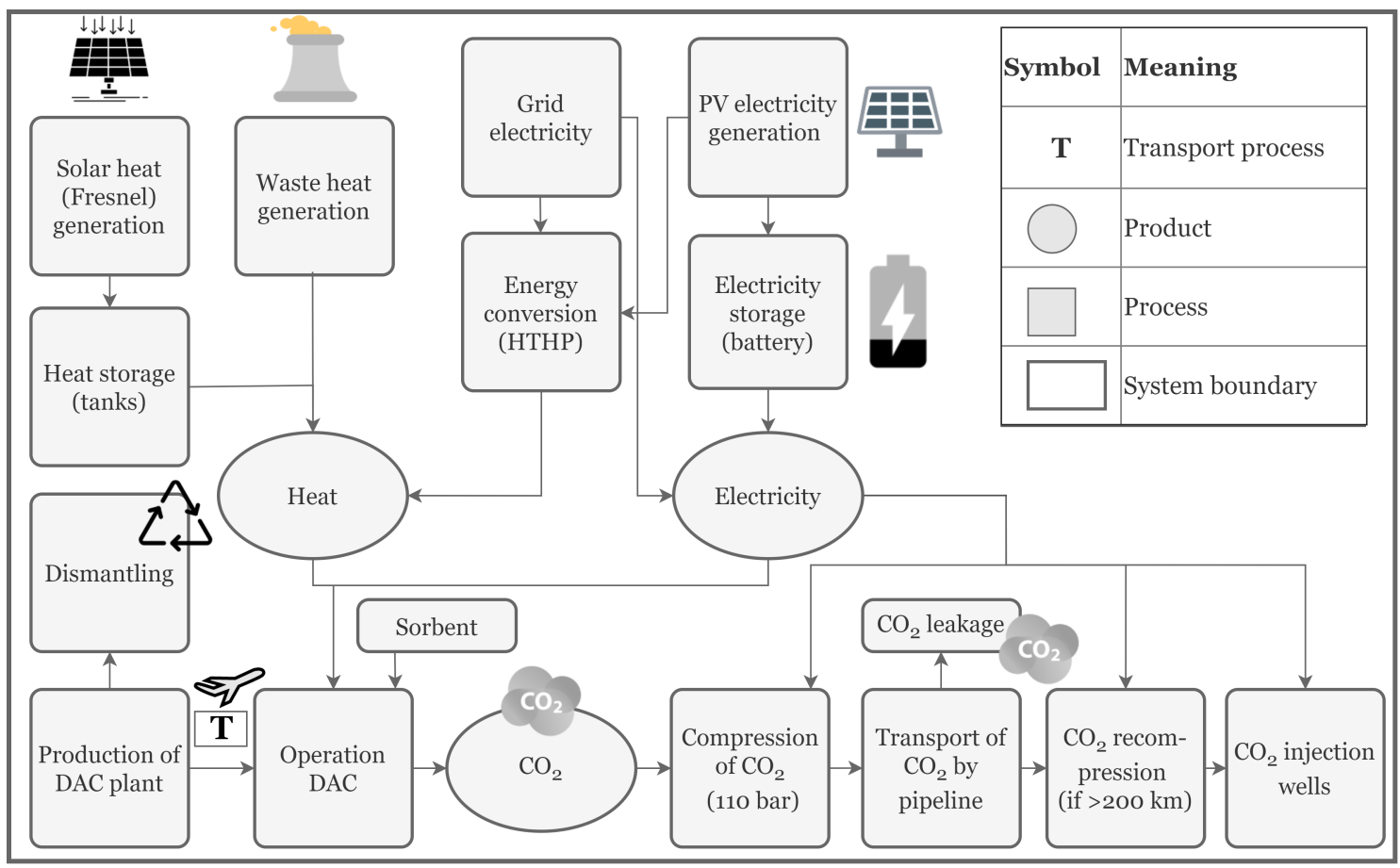

Figure 1: System boundaries of the DACCS product system. Note the different electricity and heat system layouts in the upper part of the figure. All upstream and downstream materials, services and emission flows are included, but are not shown in this figure to reduce complexity. $\mathrm{HTHP}=$ high temperature heat pump, DAC = direct air capture.

\section{Methodology: Life Cycle Assessment}

LCA is a methodology which aims to quantify environmental impacts of a product or service over its entire life-cycle. ${ }^{15,16}$ LCA is standardized by the international organization for standardisation (ISO), where ISO 14040 describes the general principles and framework of $\mathrm{LCA}^{24}$ and ISO 14044 presents guidelines and general practices for LCA. ${ }^{25}$

\subsection{Goal and Scope}

Our goal is to quantify the environmental impacts and to determine environmental hotspots (i.e. main drivers and processes determining the environmental performance) along the DACCS life-cycle considering low-carbon energy sources for $\mathrm{CO}_{2}$ capture in different DACCS system layouts. Our work focuses on innovative low-carbon energy sources and electrification 
of DACCS systems for the following reasons. Previous analysis of DAC(CS) demonstrated that impacts on climate change mainly depend on the carbon-intensity of electricity and heat sources. ${ }^{17,18,26}$ However, the overall environmental impact of the integration of low carbon energy sources - using a wide set of environmental impact categories - has not been well examined until now. Further, we expect that the penetration of low carbon energy sources and the electrification of energy systems will expand further in the future. ${ }^{22}$ And finally, site dependent boundary conditions - such as the lack of waste heat, geological $\mathrm{CO}_{2}$ storage sites or low-carbon electricity from the grid - might require novel system designs including heat and electricity storage allowing for autonomous DAC operation in proximity of geological $\mathrm{CO}_{2}$ storage sites.

In our analysis, we consider the following countries, which exhibit different climates: Greece, Mexico, Jordan, Spain and Chile are included as (semi)-arid countries with high annual solar irradiation. Besides, cooler and temperate climate regions are covered with Norway, Iceland and Switzerland. We have selected these countries based on their geological storage potentials, ${ }^{27}$ difference in grid electricity mix, ${ }^{28}$ climate variations and data availability for the fresnel solar collector. A comprehensive argumentation for the selection of these countries is provided in the supplementary information (SI) Note S2.

Our functional unit is defined as 'Gross removal of 1 ton $\mathrm{CO}_{2}$ from the atmosphere via the use of a DAC plant combined with geological $\mathrm{CO}_{2}$ storage, with a reference flow of a DAC unit removing $100 \mathrm{kt} \mathrm{CO}_{2}$ year $^{-1}$ with varying system layouts and electricity and heat inputs as specified in the individual scenarios (see Section 2.2.1). Consequently, total GHG emissions produced from all upstream and downstream DACCS activities should be less than 1 ton $\mathrm{CO}_{2}$-eq. to result in net negative $\mathrm{GHG}$ emissions, i.e. net $\mathrm{CO}_{2}$ removal from the atmosphere.

We identify no multi-functionality of our DACCS system, since the main purpose is to remove $\mathrm{CO}_{2}$ from the atmosphere in a permanent way. Hence, allocation or system expansion is not required. ${ }^{29}$ 
We use the ILCD 2.0 (2018) life cycle impact assessment (LCIA) method ${ }^{30}$ to assess the environmental performance of the proposed DACCS system layouts. We adopt 16 midpoint categories from ILCD in the protection areas climate change, ecosystem quality, human health, and resources. Further, we add one additional impact category to capture water consumption with the Water Depletion impact category of the ReCiPe 2016 LCIA methodology (1.1 (20180117)). ${ }^{31,32}$ Finally, we are interested in the total amount of land transformed associated with different DACCS configurations, since we include different PV-coupled DACCS configurations which could be land-intensive. ${ }^{4,14}$ Therefore, we aggregate all life cycle inventory flows associated with land transformation $\left(\right.$ in $\left.\mathrm{m}^{2}\right)$, since the considered environmental impact category 'land use' of the ILCD 2.0 LCIA method represents land use impacts in terms of points and as such does not lead to meaningful land transformation surface areas. Results are shown for impacts on climate change in the main text, while the complete set of results is shown in SI Note S6.

An attributional LCA perspective with the ecoinvent database (v3.6, system model Allocation, cut-off by classification ${ }^{33,34}$ ) as source of background inventories is applied. The open-source Python package Brightway2 is used to conduct our LCA. ${ }^{35}$ Our LCIs and corresponding assumptions will be discussed in Section 2.2 and Section 2.2.1. In addition to the analysis for the specific countries listed above, a sensitivity analysis shows GHG emissions of an all-electric system layout for 144 countries on a world map, see Section 3.3.2.

\subsection{System Boundaries and Technical Description}

We evaluate five different DAC configurations with specific supply of heat and electricity for $\mathrm{CO}_{2}$ capture:

(1) Electricity from the grid, heat from a waste heat source.

(2) Electricity from the grid, heat from a HTHP operated with electricity from the grid.

(3) Electricity from a PV installation, heat from a fresnel solar-thermal heat collector. 
(4) Electricity from a PV installation, heat from a HTHP operated with electricity from the PV installation.

(5) Electricity from a PV installation, heat from a waste heat source.

These configurations are detailed in subsequent sections. A simple representation of our system boundaries is shown in Fig. 1. The DACCS product system includes - depending on the specific system configuration - the production and transportation of system components, energy generators and storage units, such as the DAC plant, a fresnel heat plant, PV systems, heat storage tanks and batteries as well as transport and injection of $\mathrm{CO}_{2}$ and business flights (the so-called "foreground processes"). Detailed assumptions regarding the infrastructure and dismantling of the DAC plant (based on Climeworks' technology), sorbent used for $\mathrm{CO}_{2}$ capture, business trips as well geological storage of $\mathrm{CO}_{2}$ are provided in the SI Note S5.

Energy requirements for $\mathrm{CO}_{2}$ capture are estimated on $500 \mathrm{kWh} \mathrm{t}_{\mathrm{CO} 2}^{-1}$ for electricity (without electricity consumption for $\mathrm{CO}_{2}$ compression) and $1500 \mathrm{kWh} \mathrm{t}_{\mathrm{CO} 2}^{-1}$ for heat (at around $\left.100^{\circ} \mathrm{C}\right) .{ }^{18}$ Earlier DAC(CS) LCAs showed that energy consumption for the $\mathrm{CO}_{2}$ capture can be perceived as the crucial process in terms of environmental impacts. ${ }^{17,18,26}$ Therefore, we evaluate system layouts with different energy sources for the $\mathrm{CO}_{2}$ capture process. We focus on solar energy, waste heat and all-electric system layouts for the following reasons. First, solar energy is one of the fastest growing renewable energy sources with large potential for further expansion and comparatively low costs and can be used for both heat and electricity production. ${ }^{36}$ Second, waste heat - if available - can be considered as the optimal heat source due to its low cost and the fact that it comes (almost) burden-free in terms of environmental impacts. ${ }^{8,34}$ Third, further low-carbon electrification of future energy systems can be expected. ${ }^{22}$

Large-scale and economically attractive implementation of DACCS might, however, require remote installations close to proper sites for geological $\mathrm{CO}_{2}$ storage. Therefore, we introduce two autonomous off-grid system layouts entirely based on solar energy. Besides, 
three grid-coupled alternatives are considered. DAC operators generally aim for renewablesbased energy sources and low-carbon DAC operation, so that exclusively low-carbon grid electricity is an option for them. An overview of the different system layouts (system components, capacities, lifetimes) is given in SI Note S3. The common system lifetime is indicated as 20 years - which is the lifetime of the DACCS unit. In case of a longer lifetime of a system component, we assign a proportional fraction of the inventory of the system component to the DACCS system by dividing the common system lifetime with the lifetime of the system component. An overview of the main parameters used in the DACCS system configurations in provided in Table 1.

Table 1: Main parameters per DACCS configuration. More information per system configuration is provided in SI Note S3.

\begin{tabular}{|c|c|c|c|c|c|c|}
\hline $\begin{array}{l}\text { Main parameters } \\
\text { Storage medium }(s)\end{array}$ & $\begin{array}{l}\text { Autonomous } \\
\text { (Fresnel + PV) } \\
\text { Heat }+ \text { Battery }\end{array}$ & $\begin{array}{l}\text { Autonomous } \\
(\text { HTHP }+ \text { PV) } \\
\text { Battery }\end{array}$ & HTHP + Grid & Waste heat + Grid & $\begin{array}{l}\text { Waste heat + PV } \\
+ \text { Battery } \\
\text { Battery }\end{array}$ & Unit \\
\hline Capture capacity & 100 & 100 & 100 & 100 & 100 & kt $\mathrm{CO}_{2}$ year-1 $^{-1}$ \\
\hline Lifetime & 20 & 20 & 20 & 20 & 20 & Years \\
\hline $\begin{array}{l}\text { Electricity consumption } \\
\text { (for } \mathrm{CO}_{2} \text { capture, } \mathrm{HTHP} \text { and compression) }\end{array}$ & 690 & 1271 & 1132 & 614 & 690 & $\mathrm{kWh} / \mathrm{t} \mathrm{CO}{ }_{2}$ captured \\
\hline $\begin{array}{l}\text { Waste or fresnel heat consumption } \\
\text { (for } \mathrm{CO}_{2} \text { capture) }\end{array}$ & 1500 & 0 & 0 & 1500 & 1500 & $\mathrm{kWh} / \mathrm{t} \mathrm{CO}_{2}$ captured \\
\hline Sorbent consumption & 3.0 & 3.0 & 3.0 & 3.0 & 3.0 & $\mathrm{~kg} / \mathrm{t} \mathrm{CO}_{2}$ captured \\
\hline
\end{tabular}

\subsubsection{Autonomous (Fresnel + PV)}

The Autonomous (Fresnel $+P V$ ) system layout is supposed to allow for an autonomous off-grid DAC system operation entirely based on solar energy. However, solar energy is intermittent, resulting in fluctuations in power and heat output of the PV and fresnel units. ${ }^{37}$ These fluctuations are mitigated by two storage media: heat storage tanks and battery electricity storage. This system design enables an assessment based on the common functional unit with the same goal, i.e. to capture $100 \mathrm{kt} \mathrm{CO}_{2}$ annually from the ambient air. Less $\mathrm{CO}_{2}$ would be captured without a storage medium when the same DAC capacity is installed, since fluctuating electricity and heat supply would not allow for continuous DAC operation.

Solar heat can be generated with fresnel solar collectors when sufficient solar irradiation is present. ${ }^{38}$ Steam temperatures up to $400^{\circ} \mathrm{C}$ can be achieved with fresnel solar collectors, 
which makes fresnel solar heat an appropriate heat source for industrial applications as well as for DAC systems. ${ }^{39}$ For the desorption of $\mathrm{CO}_{2}$ heat at a temperature of $100^{\circ} \mathrm{C}$ is required. ${ }^{8}$ LCI of the fresnel solar collector has been generated in collaboration with Industrial Solar (Freiburg, Germany), based on an existing fresnel plant in Jordan. Detailed information and LCI of the fresnel system is provided in SI Note S4.

Site-specific annual solar irradiation is a key factor for the design and heat output of fresnel units and therefore, location specific plant designs are required. ${ }^{38}$ We use data received from Industrial Solar for eight locations in five countries with a direct normal irradiance of more than $2000 \mathrm{kWh} / \mathrm{m}^{2}$ year ${ }^{-1}$, which were comprehensively modelled regarding their techno-economic performance. The sites considered are located in Chile (Antofagasta), Greece (Creta), Jordan (Amman), Mexico (San luis Potosí) and Spain (Tabernas).

Electricity is supplied by large scale ground mounted PV arrays. Therefore, countryspecific LCI datasets are generated and used to represent multi-Si PV modules (see SI Note S5). Further, we use a stationary battery system to store excess PV electricity during daytime in order to be consumed during night-time. We include a lithium Nickel-ManganeseCobalt oxide (NMC) battery, representing mainstream technology for stationary electricity storage today. ${ }^{40}$

We assume that the NMC battery is able to store 12 hours of the electricity load to provide sufficient electricity during night for $\mathrm{CO}_{2}$ capture and compression, in line with the heat storage sizing and the aim to capture $100 \mathrm{kt} \mathrm{CO}_{2} \mathrm{year}^{-1}$. The minimum battery storage capacity requirement $\left(\mathrm{C}_{\text {bat,req }}\right)$ is calculated to capture $100 \mathrm{kt} \mathrm{CO}_{2}$ year $^{-1}$ considering 12 hours of storage. We oversize the NMC battery to consider battery degradation. ${ }^{41}$ Hence, the battery capacity $\left(\mathrm{C}_{\mathrm{bat}}\right)$ is determined by considering the Depth of Discharge $(\mathrm{DoD})$, the discharge efficiency $\left(\eta_{\text {dis }}\right)$ and the percentage of the original storage capacity left, required at the end of its lifetime (EoL). We use a DoD of 93\%, a discharge efficiency of $94.3 \%$ and an EoL capacity percentage of $80 \% \cdot{ }^{37}$ Equation 1 is used to size the energy capacity of the 
NMC battery. ${ }^{37}$

$$
\mathrm{C}_{\mathrm{bat}}=\frac{\mathrm{C}_{\mathrm{bat}, \mathrm{req}}}{\eta_{\mathrm{dis}} \cdot \mathrm{DoD} \cdot \mathrm{EoL}}
$$

Besides, we assume a C-rate of $0.5 \mathrm{C}$ (i.e. two hours for battery charge or discharge) as most appropriate, since the NMC battery is installed in (semi)-arid locations with high PV power peaks. Consequently, a high power capacity could be beneficial to charge during PV power peaks in order to avoid curtailment of PV electricity. Further, we consider additional electricity needed for system layouts with battery deployment to compensate for the Roundtrip Efficiency (RE) related losses of the battery. Hence, the required PV electricity is divided by the RE (i.e. $89 \%$ ) of the NMC battery to function as a safety factor. ${ }^{37}$ The latter assumption can be perceived as a worst case scenario in order to be conservative, assuming that all produced PV electricity will go through the NMC battery to be used for electricity for the DAC plant.

\subsubsection{Autonomous (HTHP + PV)}

The Autonomous $(H T H P+P V)$ system layout is an all-electric off-grid system entirely supplied by PV electricity, including a HTHP to deliver high temperature heat for the $\mathrm{CO}_{2}$ capture process. Hence, the difference with our previous system layouts is the replacement of solar heat with heat produced by a HTHP. Further, an NMC battery is used to store PV electricity during the night; the storage capacity of the battery is calculated using equation 1. Note that the battery storage capacity is larger compared to Autonomous (Fresnel $+P V)$, due to the larger electricity requirement for this all-electric system layout. We assume a coefficient of performance (CoP) of 2.9 for the HTHP, which is conservative and at the lower range of presented CoPs of HTHPs. ${ }^{42} \mathrm{CO}_{2}$ capture via DAC requires heat at relatively high temperatures $\left(100^{\circ} \mathrm{C}\right)$, compared to heat temperatures provided by HTHP on the market today, hence a $\mathrm{CoP}$ at the lower end of the range seems reasonable. ${ }^{42} \mathrm{LCI}$ of the HTHP has been generated by linearly scaling up a $10 \mathrm{~kW}$ heat pump from the ecoinvent database, to the appropriate heat pump size $(17 \mathrm{MW})$ to deliver sufficient instant heat for $\mathrm{CO}_{2}$ capture. We 
modify the LCI of the heat pump and use $\mathrm{CO}_{2}$ (R744) as refrigerant - instead of R134 - based on information from MAN Energy Solutions (Zürich, Switzerland), to represent current and future industrial practices of the HTHP industry. ${ }^{43}$

\subsubsection{HTHP + Grid}

The all-electric HTHP + Grid system layout contains a HTHP connected to the electricity grid. The same assumptions for the HTHP are used as in the previous system layout. Other energy sources are not required, since grid electricity is available in all selected countries. Consequently, the environmental impact of energy consumption (predominantly) depends on the national grid electricity mix and the performance of the HTHP. Country-specific LCI datasets of the ecoinvent database are used for grid electricity. ${ }^{34}$ We conduct a sensitivity analysis for future electricity mixes, see Section 2.3.2.

\subsubsection{Waste heat + Grid}

The Waste heat + Grid system layout consumes (industrial) waste heat and is connected to the electricity grid. Note that waste heat comes (almost) burden-free in the Allocation, cut-off by classification system model of ecoinvent. ${ }^{34}$ The Waste heat + Grid system layout is only applicable when waste heat at the correct temperature level is available for example generated from industrial processes. Therefore, a location specific assessment is required to identify the potential of waste heat. For simplicity, we decided to include all 8 countries with country-specific LCI from the ecoinvent database for waste heat and grid electricity. ${ }^{34}$

\subsubsection{Waste heat $+\mathrm{PV}+$ Battery}

The Waste heat $+P V+$ Battery system layout consumes PV electricity and waste heat as energy sources for $\mathrm{CO}_{2}$ capture. For waste heat, the same assumptions are used as in the previous system layout. Assumptions for the provision of PV electricity and battery storage are adopted from the Autonomous (Fresnel $+P V$ ) layout. Complete LCIs of all system 
layouts are provided in SI Note S5.

\subsection{Sensitivity Analysis}

\subsubsection{Reduced electricity consumption}

DACCS is an emerging technology and will profit from technological improvements. ${ }^{8}$ This could result in a reduction of energy consumption during the $\mathrm{CO}_{2}$ capture process. Current figures used for energy consumption (500 kWh t-1 $\mathrm{CO}^{-1}$ electricity and $1500 \mathrm{kWh} \mathrm{t}_{\mathrm{CO} 2}^{-1}$ heat $^{18}$ ) are based on a very high $\mathrm{CO}_{2}$ purity in the resulting $\mathrm{CO}_{2}$ stream. ${ }^{8}$ However, a lower $\mathrm{CO}_{2}$ purity seems to be compatible with carbon capture and storage (contrary to carbon capture and utilization applications), which might result in lower energy requirements. Therefore, we examine the performance of the proposed system layouts with an electricity consumption of the $\mathrm{CO}_{2}$ capture process reduced by $20 \%$.

\subsubsection{Electrification: HTHP + Grid alternative}

The all-electric HTHP + Grid system layout is further examined on a global scale to determine GHG emissions (i.e. Climate Change) of DACCS in 144 countries. We specifically focus on this all-electric system layout in our geographical sensitivity analysis, since energy system models predict an increase of low-carbon electrification in future energy systems. ${ }^{22}$ Besides, this system layout is the simplest and (theoretically) could be implemented in all locations with a (low-carbon) grid connection providing sufficient electricity, as grid electricity is the only energy source needed. Climeworks has committed - and probably also other DAC suppliers - to only offer carbon dioxide removal services with grid-coupled DAC systems at geographical locations with low GHG intensive grid electricity.

For simplicity, we exclude environmental impacts for transportation and business trips in this sensitivity analysis, since GHG emissions from transportation processes are small according to our results. Further, we use average electricity supply (i.e. 'market group for electricity, ..') for countries which are modelled with multiple regional electricity datasets 


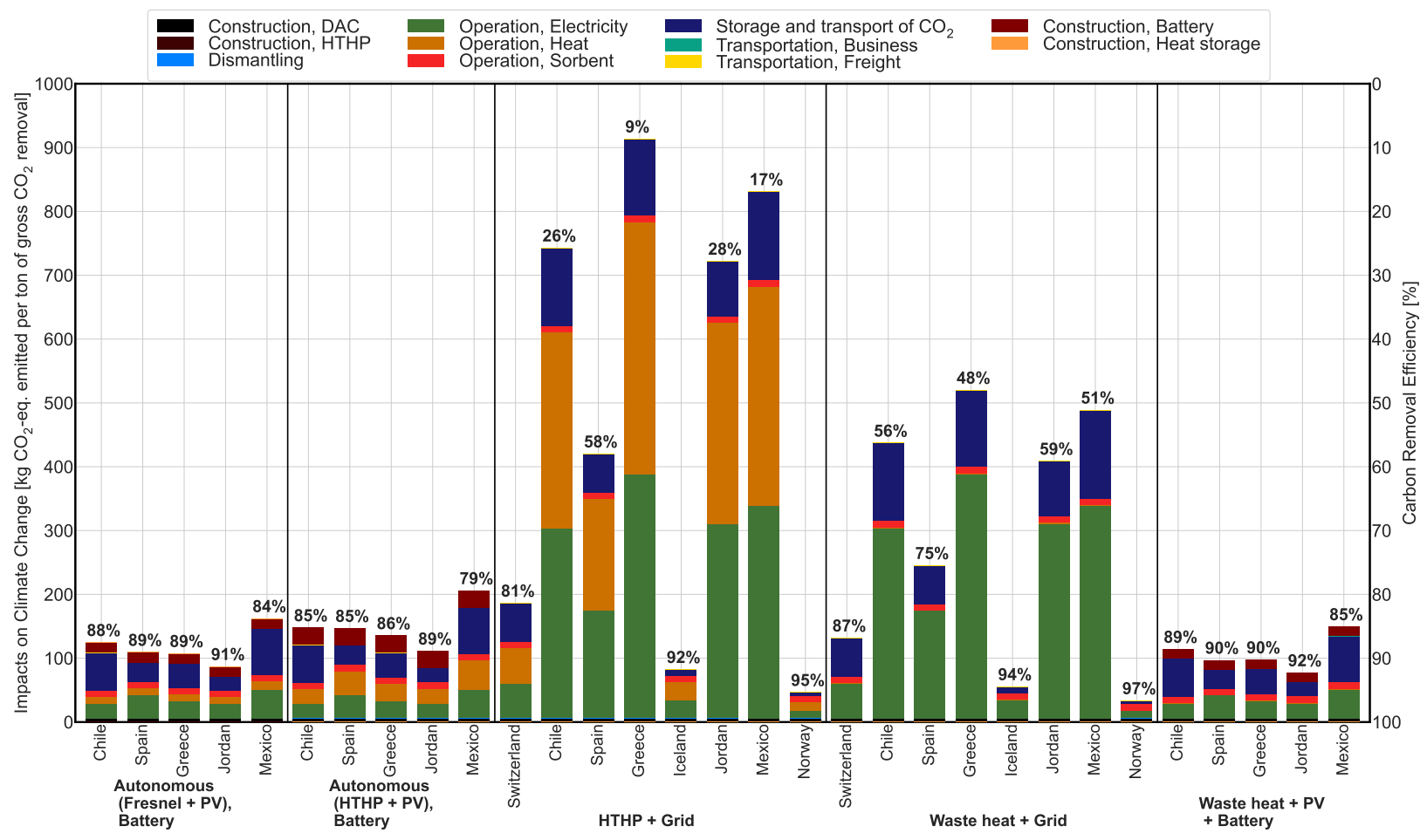

Figure 2: Life-cyle GHG emissions in $\mathrm{kg} \mathrm{CO}_{2}$-eq. per ton of gross $\mathrm{CO}_{2}$ removal with the DAC plant as well as carbon removal efficiencies [\%], for different system layouts in selected countries. The size and colors of the bar segments correspond to the contributions of specific processes to the total life-cycle GHG emissions. Note that 'Storage and transport of $\mathrm{CO}_{2}$ ' includes compression, transportation, re-compression, injection and the infrastructure requirements during the $\mathrm{CO}_{2}$ storage stage.

in the ecoinvent database. In case there is no country-specific electricity dataset available, we use the market (group) for electricity for the geographical area in which this country is located as approximation. Further, we assume a generic transportation distance for $\mathrm{CO}_{2}$ of 500 kilometers to the injection wells and a generic storage depth of 2000 meters.

\subsubsection{Future electrification: HTHP + Grid alternative}

Projections of IAMs show that electricity grid mixes will become less $\mathrm{CO}_{2}$-intensive, even in the most carbon intensive energy scenarios. ${ }^{21,22}$ Therefore, we examine GHG emissions of the HTHP + Grid system layout based on future grid electricity mixes for 2040. To achieve this, we use these future grid mixes for electricity supply for the DAC unit by modifying the 
ecoinvent background database with the rmnd-lca Python package, ${ }^{44}$ adapting future electricity mixes in our background database, based on the output figures of the REMIND model scenarios. ${ }^{21}$ Note that this results in geographically aggregated future electricity datasets, since the REMIND model subdivides the world into only 11 regions.

Firstly, we use the SSP2-Base energy scenario to determine future GHG emissions of the HTHP + Grid system layout. The SSP2-Base scenario is a scenario with no additional climate policy. ${ }^{21,44}$ Secondly, we include a more ambitious future climate policy with the SSP2-PkBudg1300 energy scenario, which corresponds to a maximum average temperature increase of $2^{\circ} \mathrm{C} .{ }^{44}$

\section{Results and discussion}

\subsection{Assessment of Climate Change impact}

The outcome of the assessment of the "Impacts on Climate Change" of DACCS technologies for the different system layouts and countries considered is illustrated in Fig. 2 and 3. The former figure is a bar chart plotting the climate change impact in kilograms of $\mathrm{CO}_{2}$ emitted per gross removal of 1 ton $\mathrm{CO}_{2}$ from the atmosphere via the use of a DAC plant (left vertical axis) as well as the net carbon removal efficiency (right vertical axis, increasing from top to bottom, and figure reported above each bar); each bar consists of the overall impact, whereby the life-cycle contributions of eleven different upstream and downstream components of the value chain are identified with different colors. The latter figure plots the (linear) dependence of the climate change impact (and of the carbon removal efficiency) on the GHG intensity of electricity generation for the different system layouts; the different slopes of these lines reflect the electricity demand of each specific system layout (and its corresponding impact

on Climate Change), whereas the vertical gray dashed lines indicate the GHG intensities of different electricity generators and national grid electricity mixes according to the ecoinvent 3.6 database. ${ }^{34}$ 


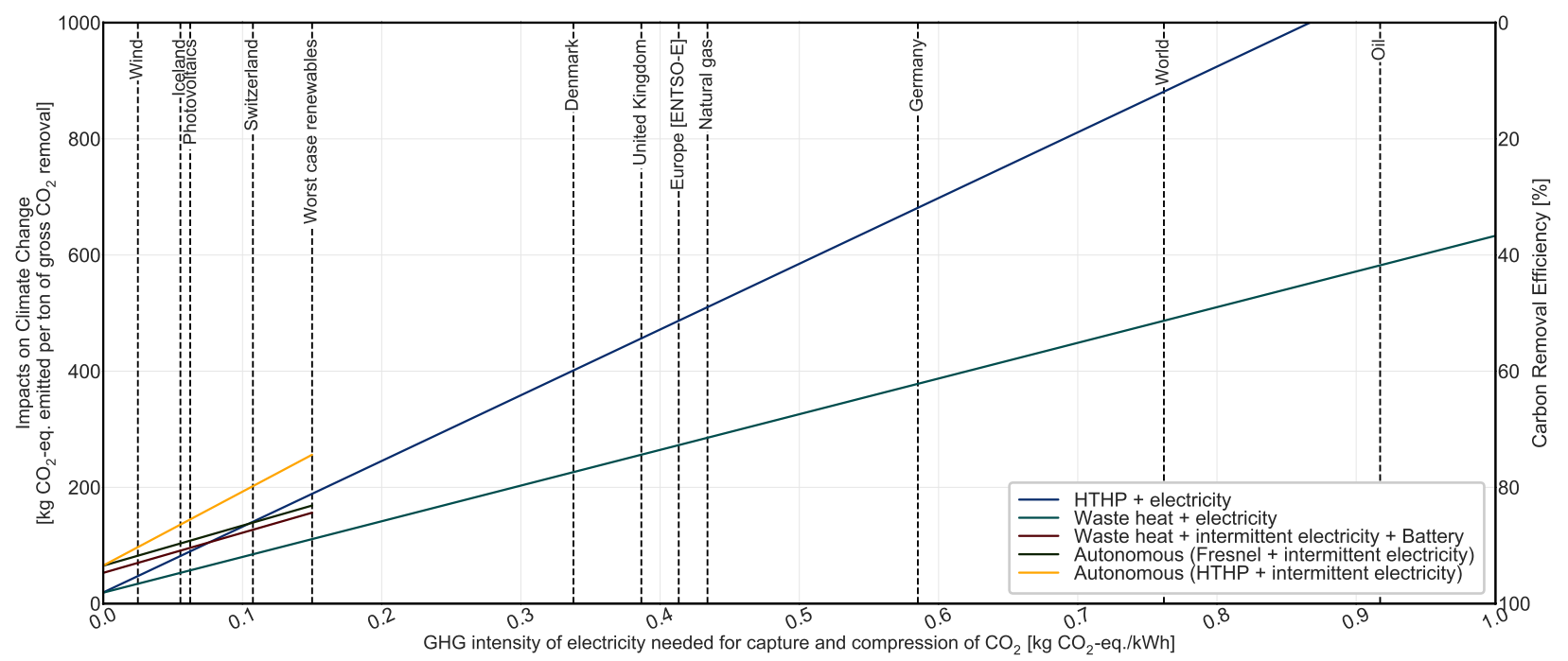

Figure 3: Effect of the GHG-intensity of electricity used - needed for capture and compression of $\mathrm{CO}_{2}$ - for all alternatives on the overall GHG emissions of DACCS systems. We use the LCI of Norway as proxy for HTHP + electricity and Waste heat + electricity. Further, LCI of Spain is used for the following configurations: Waste heat + intermittent electricity + Battery, Autonomous (Fresnel + electricity) and Autonomous (HTHP + intermittent electricity). Note that the Autonomous (Fresnel + intermittent electricity) alternative can only be installed in (semi)-arid locations due to the requirement of high annual solar irradiation. Besides, the waste heat alternatives can only be installed with sufficient supply of waste heat. The DAC configurations operated with intermittent electricity are shown up to a GHG intensity of electricity supply of $0.15 \mathrm{~kg} \mathrm{CO}_{2}$-eq. $/ \mathrm{kWh}$, which represents a worst case for renewables. The countries and specific power generation technologies on the x-axis represent the GHG-intensities of national electricity grids and technologies according to ecoinvent, see SI Note S9. ${ }^{34}$

Note that for all cases - considered in Fig. 2 - the climate change impact is smaller than the amount of $\mathrm{CO}_{2}$ removed and stored, i.e. negative emissions are indeed generated in all cases. However, the range of carbon removal efficiencies is large, going from only $9 \%$ all the way to $97 \%$. It is worth noting that carbon removal efficiency has a strong impact on unitary costs per net $\mathrm{CO}_{2}$ removed. If we assumed that the two value chains leading to $9 \%$ and to 97\% removal costed the same per ton $\mathrm{CO}_{2}$ removal, say 100 monetary units, then the unitary cost per net $\mathrm{CO}_{2}$ removed would be 1,111 and 103 monetary units, respectively. Besides, the carbon removal efficiency can be strongly dependent on the GHG footprint of electricity generation, as illustrated in Fig. 3. The main findings can be summarized as follows. 
Energy supply - heat and electricity used for $\mathrm{CO}_{2}$ capture - is the key factor driving GHG emissions of DACCS configurations. Waste heat is environmentally attractive as heat supplier when available, as waste heat comes (almost) burden-free in the Allocation, cut-off by classification system model. GHG emissions generated from energy consumption can be reduced by using renewable energy: in our analysis solar energy in countries with high annual solar irradiation.

The GHG-intensity of the grid electricity mix is a crucial factor for grid-coupled DACCS configurations and is very country-specific. Consequently, countries with low GHG intensive electricity mixes in combination with waste heat exhibit lowest GHG emissions, with GHG removal efficiencies of almost $97 \%$ and $94 \%$ for Norway and Iceland, respectively. On the contrary, high GHG emissions are generated - almost approaching net-positive GHG emissions in Greece with 0.91 ton $\mathrm{CO}_{2}$-eq. per ton of gross $\mathrm{CO}_{2}$ removal - for grid-coupled DACCS configurations when the national electricity grid relies on a large share of fossil fuels. Fig. 3 shows that the break-even point - in terms of net-negative GHG emissions for the grid-coupled HTHP DACCS configuration $(H T H P+G r i d)$ - is on $\sim 0.87 \mathrm{~kg} \mathrm{CO}_{2}$-eq. $\mathrm{kWh}^{-1}$ electricity used for the capture and compression of $\mathrm{CO}_{2}$.

In absolute terms, minor GHG emissions are generated from the DAC construction $(6 \mathrm{~kg}$ $\mathrm{CO}_{2}$-eq. per ton of gross $\mathrm{CO}_{2}$ removal) and sorbent consumption (10 kg per ton of gross $\mathrm{CO}_{2}$ removal). However, these components can be substantial in relative terms, if energy supply for $\mathrm{CO}_{2}$ capture is clean in terms of GHG emissions. The relative Climate Change impact of transportation processes directly related to DAC (i.e. business and freight transport, for the collectors and process units) can be considered small, with contributions of less than $1 \%$.

GHG emissions from the storage and transport of $\mathrm{CO}_{2}$ are significant - and their relative contribution fluctuate between 11-52\% - mainly driven by electricity requirements for the compression, recompression and injection of $\mathrm{CO}_{2}$ as well as $\mathrm{CO}_{2}$ leakage during long distance $\mathrm{CO}_{2}$ transport, respectively. Note that we included the Medium scenario presented in Holloway et al. ${ }^{20}$ for $\mathrm{CO}_{2}$ leakage during transportation. The contribution of $\mathrm{CO}_{2}$ leakage 

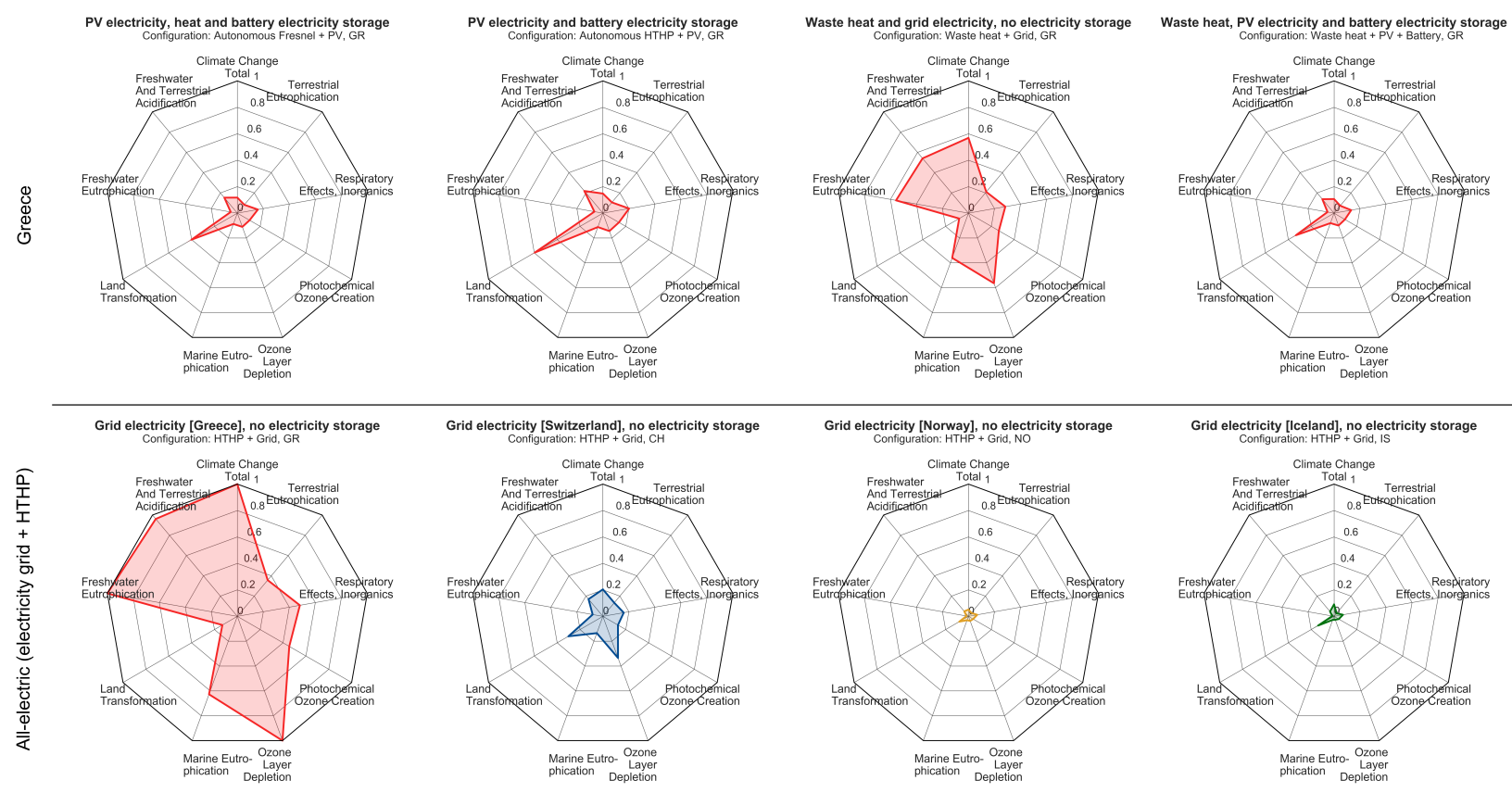

Figure 4: Spider graphs illustrating environmental trade-offs for a selection of DACCS configurations on nine environmental impact categories with a focus on Greece and all-electric DACCS configurations, please refer to the main body of the text for an explanation of this selection. Complete spider graphs considering all DACCS system configurations and environmental impact categories are provided in SI Note S6.

during the transportation of $\mathrm{CO}_{2}$ in pipelines would increase by a factor of 10 if we used their Pessimistic scenario for $\mathrm{CO}_{2}$ leakage, which emphasizes the need for a well-designed pipeline network for $\mathrm{CO}_{2}$ transportation.

DACCS-related GHG emissions in countries with a $\mathrm{CO}_{2}$-intensive grid electricity mix can be substantially reduced by shifting to renewable energy sources as energy suppliers and can be installed as (nearly) autonomous DACCS configurations. The GHG removal efficiencies of autonomous system layouts - entirely supplied by solar energy - are in between 79-91\% and are therefore at a similar - or even higher - level as grid-connected DAC in countries with rather low-carbon electricity supply and available waste heat, such as Switzerland with a GHG removal efficiency of $87 \%$.

Autonomous system layouts with a fresnel solar collector exhibit slightly higher GHG removal efficiencies (84-91\% for Autonomous (HTHP + electricity)) in comparison with 
autonomous DACCS configurations entirely based on PV arrays in combination with a HTHP (79-89\% for Autonomous (HTHP + electricity)), mainly due to the provision of lower carbon heat with a fresnel solar collector and the requirement of a smaller battery $(\sim 125$ MWh vs. $\sim 221 \mathrm{MWh}$ ). A bigger battery is required for autonomous configurations with a HTHP as electricity needs to be stored to provide sufficient electricity to the HTHP during night. Further, the production of heat storage tanks (made of low-alloyed steel) causes lower GHG emissions compared to the production of bigger battery energy storage systems, which means lower GHG emissions for DACCS configurations with fresnel heat.

The production of electricity storage (NMC battery) mediums can - in relative terms have a significant impact on GHG emissions, from $10 \%$ to $24 \%$, while that of the production of the heat storage (steel tanks) units is small with a relative contribution of less than $1 \%$. Consequently, GHG emissions from the infrastructure of autonomous systems are comparably

higher (see the lower-left part of Fig. 3), due to the impact of the production of energy storage units, such as battery energy storage systems. Fig. 3 illustrates how alternatives with waste heat consumption, and the autonomous system layout coupled with fresnel heat (Autonomous $($ HTHP + electricity)), are less sensitive when using high GHG intensive electricity, due to less electricity consumption required for $\mathrm{CO}_{2}$ capture. For example, the autonomous DACCS configuration with fresnel heat generates solar heat and therefore less electricity is required for $\mathrm{CO}_{2}$ capture, although this alternative can only be installed at locations with sufficient annual solar irradiation.

\subsection{Assessment of other environmental impact categories}

Fig. 4 illustrates the overall environmental burdens and trade-offs for a selection of DACCS configurations and environmental impact categories. The spider graphs show the normalized scores (scores from 0 to 1, visualized with the colored line) of DACCS configurations on nine environmental impact categories, normalized to the system configuration with the highest environmental impact among all configurations (i.e. highest score $=1$, presented in SI 
Note S6) on a specific environmental impact category. A greater colored area therefore demonstrates an overall higher environmental burden for a specific DACCS configuration.

Environmental impact categories - presented in Fig. 4 - are selected based on recommendations by Fazio et al. ${ }^{30}$ We only show impact categories of recommendation level I and II in the main article and add land transformation, since these are important for CDR technologies in general. ${ }^{6}$ Greece is included as a reference and worst-case scenario regarding GHG emissions. All-electric DACCS system configurations - i.e. HTHP + grid electricity - exhibit a large sensitivity regarding overall environmental impacts between countries. We therefore include three other countries with cleaner electricity mixes in terms of GHG emissions - Switzerland, Norway and Iceland - since Climeworks has committed to only offer carbon dioxide removal services with grid-coupled DAC systems at geographical locations with low GHG intensive grid electricity. The complete set of spider graphs and results for all DACCS configurations and environmental impact categories is provided in SI Note S6.

In general, lowest overall environmental burdens can be achieved with DACCS configurations with waste heat in combination with renewable electricity supply, for example in Norway and Iceland. Fundamental differences between environmental impact categories and countries are found for grid-coupled DACCS configurations in combination with a HTHP. This is illustrated in the second row of Fig. 4. Low overall environmental burdens can also be achieved with grid-coupled DACCS configurations - in combination with a HTHP (Autonomous (HTHP + electricity) $)$ - when the national grid electricity mix is based on a large-share of renewables as for example in Norway and Iceland, which both rely on a high share of hydropower. Switzerland has slightly higher overall environmental burdens compared to Norway and Iceland, mainly due to a higher reliance on nuclear power and fossil fuels, respectively.

On the contrary, substantially higher environmental burdens are generated from gridcoupled DACCS configurations when the national grid electricity mix is based on a large share of fossil fuels as for example in Greece (see Fig. 4). This generally results in high 
environmental burdens on almost all environmental impact categories.

Autonomous DACCS configurations generate low environmental burdens on almost all environmental impact categories, although several environmental trade-offs occur. The most important one concerns land use, which can be considered as the main "outlier" among all the impact categories: Autonomous DACCS layouts with solar energy supply perform in general worse than grid-connected systems on land transformation (see the first 2 spider graphs in the top row of Fig. 4). Fig. E - in SI Note S7 - presents a detailed contribution analysis on land transformation (in $\mathrm{m}^{2}$ ), and reveals that autonomous energy systems exhibit large land transformations (up to $1.87 \mathrm{~m}^{2}$ per ton of gross $\mathrm{CO}_{2}$ removal), mainly due to large surface area requirements for ground mounted PV panels and the fresnel heat collector, while the DAC unit as such exhibits very low land transformation impacts (a life-cycle land transformation of 0.01 per ton of gross $\mathrm{CO}_{2}$ removal). In addition, autonomous energy systems exhibit comparatively high environmental scores on the Minerals and Metals impact category (see SI Note S6), mainly resulting from material requirements for the production of PV arrays and the NMC battery. Note that this impact category is associated with substantial uncertainties and also methodological shortcomings, and is therefore assigned with recommendation level III. ${ }^{30}$

These examples demonstrate the importance to assess and compare system layouts on a wide set of environmental impact categories to examine environmental trade-offs.

\subsection{Sensitivity Analysis}

\subsubsection{Reduced electricity consumption}

Fig. E - in SI Note S8 - demonstrates the absolute change in the Climate Change impact category when the electricity consumption for the $\mathrm{CO}_{2}$ capture process is reduced by up to $20 \%$. Such an efficiency improvement would result in substantial reductions of impacts on Climate Change for system layouts which consume large amounts of $\mathrm{CO}_{2}$-intensive electricity (i.e. HTHP + Grid in Greece and Mexico). In contrast, the environmental impact of such 
a electricity reduction is hardly visible for configurations using low-carbon energy sources. Nevertheless, reducing pressure on the electricity grid requires increased efficiency of any technology, including DACCS.

\subsubsection{Electrification: HTHP + Grid alternative}

Fig. 5 illustrates the performance on the Climate Change impact category for the HTHP + Grid system layout for 144 countries. Net-negative GHG emissions can be obtained when life-cycle GHG emissions are lower than $1000 \mathrm{~kg} \mathrm{CO}$-equivalent per ton of gross $\mathrm{CO}_{2}$

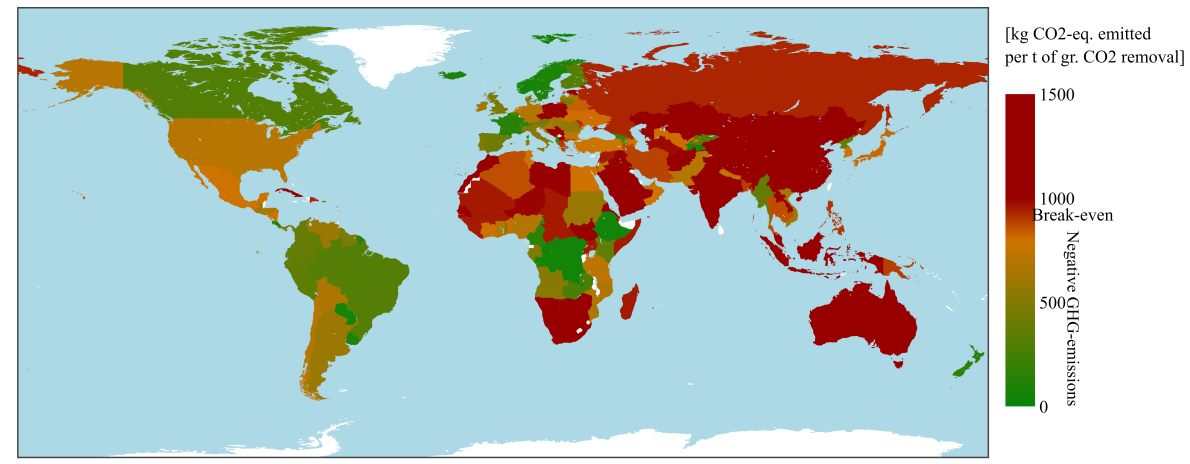

Figure 5: Country-specific results for the Climate Change impact category for the HTHP + Grid system configuration. Green and orange indicate net-negative GHG emissions, while dark red shows net-positive GHG emissions of DACCS.

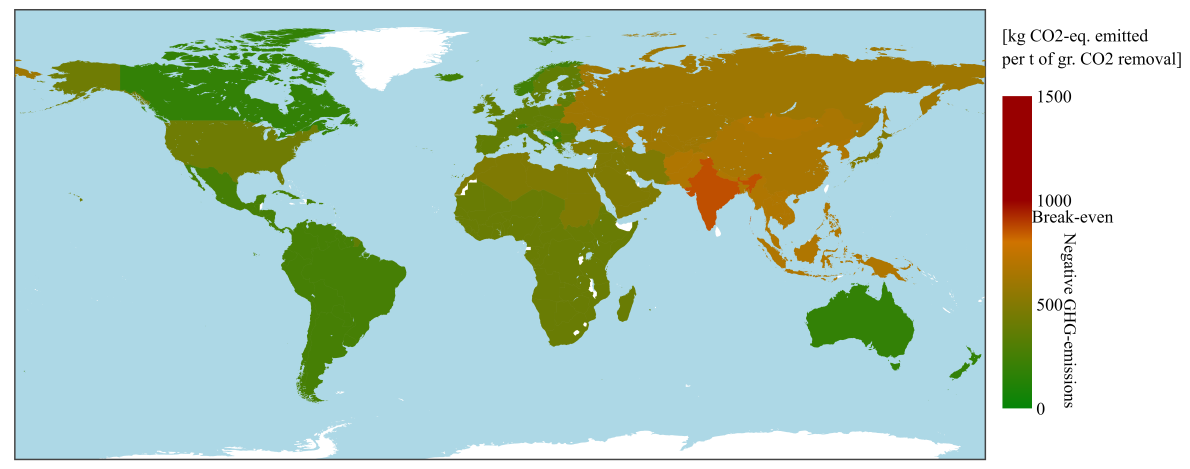

Figure 6: Results for the Climate Change impact category for the HTHP + Grid alternative, according to the SSP2-Base scenario. Green and orange colors indicate net-negative GHG emissions, while dark red shows net-positive GHG emissions. 
removal. Countries which fulfill this requirement are shaded in green and orange (i.e. orange meaning higher GHG emissions than green). Net positive GHG emissions are shown in dark red.

Significant variations between countries can (mainly) be explained by the difference of the $\mathrm{CO}_{2}$-intensity of national grid electricity mixes. For the $H T H P+$ Grid system layout, the GHG break-even point is reached with a grid electricity mix GHG intensity of $\sim 0.87 \mathrm{~kg}$ $\mathrm{CO}_{2}$-eq. $\mathrm{kWh}^{-1}$ electricity (see also Fig. 3 for the break-even point) - which means that grid electricity mixes with a lower GHG intensity than the GHG break-even point exhibits GHG removal from the atmosphere. It turns out that most countries in Europe, North-America, South-America and central Africa already show large GHG removal potentials with DACCS HTHP + Grid system layouts today. However, few countries in these continents exhibit net-positive GHG emissions. In general, Australia and countries in Asia, Southern Africa and Northern Africa are nowadays not (or less) suitable to install DACCS HTHP + Grid systems.

\subsubsection{Future electrification: HTHP + Grid alternative}

Fig. 6 demonstrates the possible future impact (in the year 2040) regarding the Climate Change impact category for the HTHP + Grid system layout according to the SSP2-Base

scenario of the REMIND model, ${ }^{21,23}$ which extrapolates observed trends, but is not particularly ambitious in terms of climate policy. It turns out that the deployment of HTHP + Grid DACCS system layouts could result in net-negative GHG emissions in all world regions in 2040. North-America, South America, Europe, Australia and Africa exhibit a large net GHG removal potential, while India and Asia seem to be less suitable due to their still relatively high $\mathrm{CO}_{2}$-intensive grid electricity mix.

When implementing a more ambitious climate scenario (i.e. SSP2-PkBudg1300) DACCS in all regions exhibits GHG emissions of less than $230 \mathrm{~kg} \mathrm{CO}$-eq. per ton of gross $\mathrm{CO}_{2}$ removal in 2040. Hence, all regions would be green if the world map for this case were drawn 
using the same color codes as used for Figures 5 and 6. Therefore, effective climate policy would be very beneficial for all-electric DACCS system layouts connected to the grid.

Note that in these two prospective evaluations we not only use future regional electricity mixes for electricity supply to the DACCS unit, but also populate the scenario-specific development of the electricity sectors throughout the entire background LCA database. This procedure results, for example, in a reduction of GHG emissions due to PV electricity, since the electricity used for PV wafer production exhibits a scenario-specific GHG intensity lower than today's.

\subsection{Discussion - limitations and future work}

Our analysis shows that within the LCA of DACCS systems, there are few elements and key factors, which determine the results: first of all, energy required for DAC operation and the sources of supply. While we consider data availability and quality regarding energy demand of and supply for, respectively, the $\mathrm{CO}_{2}$ capture process - the most crucial factor by far driving LCA results - to be high, there are other issues, which call for further analysis in the future. More sophisticated modeling integrating operational experience of DAC units of different scale and at different locations to be gained in the future will allow for a more precise representation of the environmental burdens due to DACCS. We discuss the most important of these elements in the following subsections.

\subsubsection{High Temperature Heat Pumps (HTHPs)}

We included a HTHP in different DACCS system layouts. Currently, no complete LCA of HTHP has been conducted. We received information regarding potential future refrigerants used in HTHPs from MAN Energy Solutions (Zürich, Switzerland), but had to establish most of our LCI as an extrapolation of a low-temperature HP. Future analysis should consider the utilization of different types of refrigerants, since refrigerants usually exhibit large contributions to environmental burdens caused by heat pumps. ${ }^{42,45}$ 


\subsubsection{Assessment of fresnel heat plant in other locations}

Only five potential locations for the fresnel solar collector were included in the analysis due to the site-specific performance of fresnel solar collectors and limitations on data for further locations. The fresnel performances at these locations in semi-arid climate regions were thoroughly modelled by Industrial Solar (Freiburg, Germany). Since the integration of fresnel solar heat systems turned out to be beneficial from the environmental perspective, especially for autonomous DACCS systems, site-specific assessments and modelling should be expanded to determine the environmental merits in other geographical locations.

\subsubsection{Competition between electricity sources}

Future energy systems tend to show higher reliance on electricity than current energy systems. Additional electricity will be required by the mobility and heat sector, possibly on top of increased power demand due to population growth and higher electrification rates. Increasing the pressure on the power sector through the installation of grid-coupled DACCS systems competes with other demands of electricity. This was not a topic of our research, but has to be considered.

\subsubsection{Battery storage in (semi)-arid countries}

We proposed to install NMC batteries in (semi)-arid countries (i.e. Chile, Spain, Greece, Jordan and Mexico) with high ambient air temperatures. Temperature levels within these countries could easily reach more than $40^{\circ} \mathrm{C}$ during warm periods. The acceptable temperature range for lithium-ion batteries is between $-20^{\circ} \mathrm{C}$ and $60^{\circ} \mathrm{C}$, with an optimal operation temperature between $15^{\circ} \mathrm{C}$ and $35^{\circ} \mathrm{C}$. ${ }^{46}$ Therefore, location-specific measures should be considered when outside temperatures approach critical temperature levels to avoid battery damage. These measures could result in additional energy and/or material requirements, i.e. higher environmental impacts. 


\subsubsection{Autonomous system layouts}

Two autonomous system layouts - entirely based on solar energy supply - were included: Autonomous (Fresnel $+P V)$, and Autonomous (HTHP $+P V)$. Twelve hours of energy storage capacity was assumed for storage mediums for these two system layouts. This led to a large storage capacity needed for both the battery system $(\sim 125 \mathrm{MWh}$ and $221 \mathrm{MWh}$, respectively) and the heat storage tanks.

Alternatively, a doubling of the DAC capacity (in order to comply with our functional unit) with discontinued operation over night could be installed to reduce the need for energy storage in the proposed two autonomous system layouts. However, we emphasize that such a system with doubled DAC capacity would still require energy storage for intermittent (renewable) electricity generation, which would result in additional environmental impacts from the production of storage mediums. Further, a doubling of the capacity of the DAC plant would incur large additional capital expenditures for the DAC plant, since capital expenditures of DAC systems are still high. ${ }^{8}$ Therefore, we argue that such a DAC system is currently unrealistic from an economic and technological point of view - and further research is required for a complete assessment of the operation of such an autonomous DAC system.

Further, more sophisticated research is needed to confirm the self-sufficiency - e.g. the 12 hours of energy storage - of the proposed autonomous system layouts. For the Autonomous $H T H P+P V$, the environmental and economic performance can possibly be (slightly) improved with the installation of a heat storage medium to reduce the size of the battery. In this way, heat supply - needed for the $\mathrm{CO}_{2}$ capture process - can be stored in a heat storage medium instead of electricity storage in a battery.

In addition, autonomous system designs with DAC units entirely powered by solar energy require site-specific assessments in terms of land use. The area covered by PV arrays required for electricity supply for a DAC unit removing $100 \mathrm{kt} \mathrm{CO}_{2}$ year $^{-1}$ in our most land-intensive case and location (i.e. Autonomous HTHP $+P V$ in Mexico) amounts to a direct land transformation (for the DAC unit and PV panels considering their lifetimes) of almost 4.7 
$\mathrm{km}^{2}$. Such a land surface area will not always be available. The scale up of solar PV-coupled

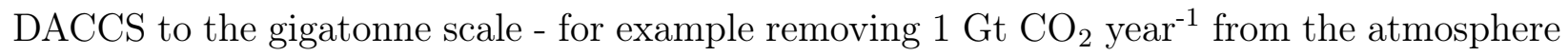
with a GHG removal efficiency of $79 \%$ (representing worst case Autonomous HTHP + PV for Mexico) - would therefore require a direct land transformation area as large as $\sim 59,000$ $\mathrm{km}^{2}$; which is almost 1.5 times the total land surface area of Switzerland.

Land surface area requirements can be significantly reduced by for example using grid electricity sources, although this would result in indirect land use transformations mainly generated from electricity generators. Note that the land transformation for PV panels includes area requirements for the open ground power plant including the space in between the modules as well as some surrounding area, ${ }^{34}$ and can therefore be considered as conservative. Further, the land between and underneath the PV modules may be used for grazing of small livestock such as sheep or poultry, bee-keeping, or other innovative agricultural uses. Such land use options should already be considered during the design phase of the solar field.

\subsubsection{Location-specific limitations}

Location-specific assessments are required to identify the availability of waste heat from industrial applications. For example, waste heat could be inaccessible in remote locations, which would limit the design options. Furthermore, decarbonization of the energy system with reduced combustion of fossil energy carriers might result in a reduction of waste heat sources in general, and/or at the required temperature level. However, grid-coupled and autonomous DACCS energy systems provide - in environmental terms - a good alternative for waste heat in these situations.

Another site-specific boundary condition of DACCS is the availability of geological storage sites. We therefore examined different transportation distances from $\mathrm{CO}_{2}$ capture to the injection wells, although the transportation distance of $\mathrm{CO}_{2}$ can be minimized - to avoid $\mathrm{CO}_{2}$ leakages from the transportation of $\mathrm{CO}_{2}$ - by installing the DAC facility close to geological storage sites. However, the large scale deployment of DACCS requires large geological 
storage potentials and therefore $\mathrm{CO}_{2}$ transportation might be required. This could result in competition for geological storage sites between DACCS and for example carbon capture and storage from conventional power plants, which could limit the large-scale implementation of DACCS.

\subsubsection{Future electrification: REMIND regions}

We used the outputs of the IAM REMIND ${ }^{21}$ for quantification of future GHG-intensity of electricity supply and to modify our background LCA database to assess the future performance of all-electric DACCS systems. However, the geographical resolution of the REMIND model is limited to 11 world regions. Therefore, the future environmental potential of the electrified system layouts had to be aggregated to those regions, while our results for current systems showed that regional differences can be significant in terms of GHG emissions (see Fig. 5). Further geographical disaggregation would be beneficial for prospective LCA of DACCS, especially for all-electric system layouts. However, our findings demonstrate very well how effective climate policy could improve the environmental performance of all-electric DACCS systems. On the other hand, the decarbonization of the power supply system could lead to additional environmental impacts on other life-cycle environmental impact categories. Luderer et al. ${ }^{47}$ investigated benefits and side-effects of the decarbonization of power supply.

\subsubsection{Comparison with literature on DACCS}

The single purpose of DACCS systems is to remove $\mathrm{CO}_{2}$ from the atmosphere in a permanent way. Our analysis focused on LT DACCS systems and must not be considered as representative for HT DACCS systems, due to the need of different processes for $\mathrm{CO}_{2}$ capture and their associated energy requirements. ${ }^{8,48}$

One recent DACCS LCA study of Deutz and Bardow ${ }^{18}$ examined the same LT DAC technology of Climeworks although focused on a smaller DAC plant (with a capture capacity of $4 \mathrm{kt} \mathrm{CO}_{2}$ year-1), and therefore provided less detailed results for the larger DAC plant 
(with a capture capacity of $100 \mathrm{kt} \mathrm{CO}_{2}$ year $^{-1}$ ). Their GHG removal efficiencies are generally slightly higher compared to the reported GHG removal efficiencies in this work. For example, Deutz and Bardow ${ }^{18}$ found a carbon removal efficiency of $88.8 \%$ for Switzerland - consuming the same amount of grid electricity and waste heat as energy sources for $\mathrm{CO}_{2}$ capture as our analysis - for the $4 \mathrm{kt} \mathrm{CO}_{2}$ year $^{-1} \mathrm{DAC}$ facility. The $\mathrm{CO}_{2}$ removal efficiency can be expected to be higher for the $100 \mathrm{kt} \mathrm{CO}_{2}$ year $^{-1}$ DAC facility, due to reductions of sorbent consumption as well as the optimization of the DAC infrastructure (see Section 2.2 and SI Note S1). Reported GHG emissions of the carbon storage phase turned out to have a minor contribution with less than $2 \%$ on total GHG removal efficiencies for DACCS configurations with GHG removal efficiencies higher than $86 \%$.

On the contrary, our results show a lower GHG removal efficiency of $86.8 \%$ for the waste heat and grid electricity configuration (Waste heat + Grid) in Switzerland; (even) for a DAC plant with a capacity of $100 \mathrm{kt} \mathrm{CO}_{2}$ year $^{-1}$. First, this can be partly explained by the application of different background LCA data; ecoinvent 3.6 in our analysis vs. both Gabi and ecoinvent 3.5 in Ref. ${ }^{18}$ Second, our analysis includes a more thorough (and conservative) analysis regarding the transportation and storage of $\mathrm{CO}_{2}$, while Ref. ${ }^{18}$ expected sufficient $\mathrm{CO}_{2}$ storage sites close to the DAC facility as well as the utilization of existent infrastructure for $\mathrm{CO}_{2}$ storage; they therefore excluded $\mathrm{CO}_{2}$ transportation and infrastructure requirements for geological storage sites. However, the availability of geological storage sites for DACCS can be limited due to geographical availability as well as the competition with conventional CCS and other CDR technologies, such as BECCS (see Section 3.4.6). For Switzerland, $\mathrm{CO}_{2}$ leakage during transportation $(1500 \mathrm{~km})$ reduced the GHG removal efficiency already by more than $2 \%$, while the compression, recompression and injection of $\mathrm{CO}_{2}$ as well as infrastructure requirements further reduced the GHG removal efficiency to a total of $6 \%$ in our analysis.

In addition, the work of Deutz and Bardow ${ }^{18}$ reported a generic land use requirement

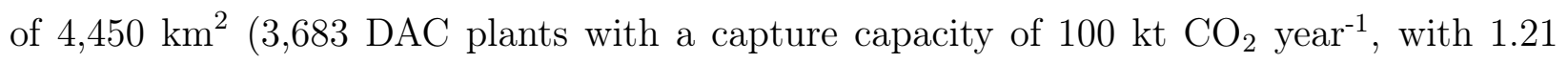


$\mathrm{km}^{2}$ per DAC plant) for PV-coupled DACCS to capture $1 \%$ of global $\mathrm{CO}_{2}$ emissions in year 2019 (0.368 Gt $\left.\mathrm{CO}_{2}{ }^{18}\right)$. Our main environmental trade-off was found for land transformation; manifested by the big range of both direct and life-cycle land transformations between DACCS configurations and countries. The worst case land transformation scenario in our analysis demonstrated much higher land use requirements for autonomous PV-coupled

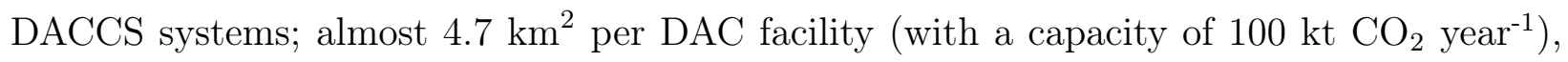
mainly due to lower annual solar irradiation for this specific location (and for the reason given in Section 3.4.5).

Since our analysis and this comparison show that specific DACCS system configurations at different locations exhibit very different environmental performances and that transport and storage of $\mathrm{CO}_{2}$ can cause substantial burdens, we argue to perform site-specific assessments of DACCS configurations during the design phase, including a thorough analysis of the transportation and storage of $\mathrm{CO}_{2}$ as well as a location-specific assessment of land use requirements.

\subsubsection{Comparison with CDR technologies}

Comparison of DAC(CS) LCA results with other CDR technologies or $\mathrm{CO}_{2}$ sources for carbon capture and utilization should consider the variability of the life-cycle GHG removal efficiency, as demonstrated by our analysis. This is, however, not always the case and the presentation of the GHG removal performance of $\mathrm{DAC}(\mathrm{CS})$ as one single number can be misleading (e.g. by Müller et al. ${ }^{29}$ for DAC).

Further, DACCS systems should be compared with other CDR technologies on the same functional unit - to evaluate benefits and potential trade-offs of various CDR technology options. ${ }^{6}$ We propose to compare CDR technologies per unit of $\mathrm{CO}_{2}$-removal from the atmosphere. Unfortunately, we are currently not able to present such a comparison between CDR technologies due to the immature research state of CDR technologies in general. ${ }^{6}$ 


\section{Conclusion}

Our paper aimed to determine the life-cycle environmental performance of low temperature DACCS systems with low carbon energy sources for $\mathrm{CO}_{2}$ capture. The results were presented per ton of gross $\mathrm{CO}_{2}$ removal with a DAC plant of an annual capture capacity of $100 \mathrm{kt}$ $\mathrm{CO}_{2}$ year $^{-1}$ based on Climeworks' technology. We included different energy sources - solar heat, waste heat and electricity by means of a HTHP - to deliver heat to the DAC plant. In addition, different electricity sources - PV and grid electricity - were considered to supply electricity to the $\mathrm{CO}_{2}$ capture process. The results were presented for different system layouts for eight geographic locations with specific assumptions regarding $\mathrm{CO}_{2}$ transport and storage after capture.

The results demonstrated that energy supply - heat and electricity used for $\mathrm{CO}_{2}$ capture - is the key factor driving GHG emissions of DACCS configurations. All eight selected countries exhibited net-negative GHG emissions on all proposed system layouts, meaning that GHG emissions associated with energy supply and material demand for DACCS are below the amount of gross $\mathrm{CO}_{2}$ removal, equivalent to a net-removal of $\mathrm{CO}_{2}$ from the atmosphere. However, the level of "net-negativity" showed substantial variation between DACCS layouts and countries of application: while in our best case almost $97 \%$ of the captured $\mathrm{CO}_{2}$ is permanently removed from the atmosphere, our worst case resulted in a life-cycle GHG removal rate of less than $9 \%$. The best climate change-related performances were achieved by system layouts using waste heat - and in countries with low $\mathrm{CO}_{2}$-intensities of the national electricity grid. The $\mathrm{CO}_{2}$-intensity of the national grid electricity mix turned out to be the crucial factor for grid-coupled system layouts.

Autonomous DACCS layouts - which consume solar energy - are from an environmental perspective (except for land use considerations) promising alternatives in regions where the grid electricity mix relies on a high share of fossil fuels and at remote locations without grid access (potentially close to $\mathrm{CO}_{2}$ storage sites). Therefore, we recommend solar-based autonomous DACCS systems for countries with (semi)-arid climates, which have a $\mathrm{CO}_{2^{-}}$ 
intensive grid electricity mix and the required land area available. All-electric DACCS system layouts are recommended when the national grid electricity mix relies on low-carbon electricity sources. Further contributions to life-cycle GHG emissions of DACCS - associated to for example DAC infrastructure, $\mathrm{CO}_{2}$ transport and storage, and energy storage units can be substantial in relative terms, if energy supply for $\mathrm{CO}_{2}$ capture is clean in terms of GHG emissions, but absolute GHG emissions due to these contributions are rather small. The assessment of a wide variety of environmental impact categories - in addition to impacts on climate change - showed different trade-offs on a few environmental impact categories, especially regarding land transformation associated with PV-coupled DACCS configurations. This observation confirms the importance of a comprehensive LCA approach not only focusing on climate change.

The sensitivity analysis demonstrated the large variation of GHG emissions between countries for all-electric DACCS systems. Hence, selecting inappropriate locations in countries with $\mathrm{CO}_{2}$-intensive grid electricity mixes could lead to net GHG emissions instead of GHG removal. Consequently, the operation of all-electric DACCS systems with fossil-fuel based grid electricity mixes should be avoided, and we recommend to assess the suitability of DACCS systems based on site-specific conditions, such as the availability of (renewable) energy sources as well as land, waste heat and the potential of carbon storage sites. Further, our prospective analysis demonstrated that more ambitious climate policy will have beneficial effects on GHG emissions for all-electric DACCS systems.

Finally, we demonstrated significant carbon removal efficiencies of DACCS, although with a large dependency on the energy sources used for $\mathrm{CO}_{2}$ capture. We therefore argue to utilize low-carbon energy sources for $\mathrm{CO}_{2}$ capture and to compare alternative DACCS configurations with a comprehensive and transparent LCA approach. Such an approach enables policy-makers to select the most environmentally friendly DACCS configuration under given local boundary conditions. Besides environmental aspects, effective policy is required to facilitate the development of the DAC industry to improve the economic viability of DACCS. 
With sufficient geological storage potentials and an economically viable DAC system, we foresee DACCS as a key component in the pathway towards net-zero GHG emissions.

\section{Conflicts of interest}

There are no conflicts to declare.

\section{Acknowledgement}

This work has mainly been funded by ACT ELEGANCY, Project No 271498, which has received funding from DETEC (CH), BMWi (DE), RVO (NL), Gassnova (NO), BEIS (UK), Gassco, Equinor and Total, and is co-funded by the European Commission under the Horizon 2020 program, ACT Grant Agreement No 691712. This project is supported by the pilot and demonstration program of the Swiss Federal Office of Energy (SFOE). Further financial support was provided by the Kopernikus Project Ariadne (FKZ 03SFK5A), funded by the German Federal Ministry of Education and Research and PSI's ESI platform.

The authors thank Daniel Sutter and colleagues from Climeworks (Zürich, Switzerland), Christian Zahler and Stephan Scherer from Industrial Solar (Freiburg, Germany), and Emmanuel Jacquemoud and Raymond Decorvet from MAN Energy Solutions (Zürich, Switzerland) for the fruitful discussions, feedback and data provision.

\section{Supporting Information Available}

The Supporting Information is available free of charge on the ACS Publications website at DOI: ... 


\section{References}

(1) Minx, J. C. et al. Negative emissions - Part 1: Research landscape and synthesis. Environmental Research Letters 2018, 13, 063001.

(2) Fuss, S. et al. Negative emissions - Part 2: Costs, potentials and side effects. Environmental Research Letters 2018, 13, 063002.

(3) Sykes, A. J. et al. Characterising the biophysical, economic and social impacts of soil carbon sequestration as a greenhouse gas removal technology. Global Change Biology 2020, 26, 1085-1108.

(4) Smith, P. et al. Biophysical and economic limits to negative CO2 emissions. Nature Climate Change 2016, 6, 42-50.

(5) Rogelj, J.; Shindell, D.; Jiang, K.; Fifita, S.; Forster, P.; Ginzburg, V.; Handa, C.; Kheshgi, H.; Kobayashi, S.; Kriegler, E.; Mundaca, L.; Séférian, R.; Vilariño, M. V. Mitigation pathways compatible with $1.5^{\circ} \mathrm{C}$ in the context of sustainable development Global warming of $1.5^{\circ} \mathrm{C}$ - An IPCC Special Report. Global Warming of $1.5^{\circ}$ C. An IPCC Special Report on the impacts of global warming of $1.5^{\circ} \mathrm{C}$ above pre-industrial levels and related global greenhouse gas emission pathways, in the context of strengthening the global response to the threat of climate change, 2018, 1-1308.

(6) Terlouw, T.; Bauer, C.; Rosa, L.; Mazzotti, M. Life cycle assessment of carbon dioxide removal technologies: a critical review. Energy Environ. Sci. 2021, 14, 1701-1721.

(7) Rosa, L.; Sanchez, D. L.; Realmonte, G.; Baldocchi, D.; D'Odorico, P. The water footprint of carbon capture and storage technologies. Renewable and Sustainable Energy Reviews 2020, 110511.

(8) Fasihi, M.; Efimova, O.; Breyer, C. Techno-economic assessment of CO2 direct air capture plants. Journal of Cleaner Production 2019, 224, 957-980. 
(9) Fuhrman, J.; McJeon, H.; Patel, P.; Doney, S. C.; Shobe, W. M.; Clarens, A. F. Foodenergy-water implications of negative emissions technologies in a $+1.5 \mathrm{C}$ future. Nature Climate Change 2020, 10, 920-927.

(10) Chen, C.; Tavoni, M. Direct air capture of CO2 and climate stabilization: A model based assessment. Climatic Change 2013, 118, 59-72.

(11) Beuttler, Christoph and Charles, Louise and Wurzbacher, Jan, The Role of Direct Air Capture in Mitigation of Anthropogenic Greenhouse Gas Emissions. Frontiers in Climate 2019, 1, 10.

(12) Keith, D. W.; Holmes, G.; Angelo, D. S.; Heidel, K. A Process for Capturing CO2 from the Atmosphere. Joule 2018, 2, 1573-1594.

(13) CarbFix2. 2020; https://www.carbfix.com/carbfix2, Accessed on Thu, June 18, 2020.

(14) Viebahn, P.; Scholz, A.; Zelt, O. The Potential Role of Direct Air Capture in the German Energy Research Program-Results of a Multi-Dimensional Analysis. Energies 2019, 12, 3443 .

(15) Hellweg, S.; Milà i Canals, L. Emerging approaches, challenges and opportunities in life cycle assessment. Science (New York, N.Y.) 2014, 344, 1109-13.

(16) Guinée, J. B.; Heijungs, R.; Huppes, G.; Zamagni, A.; Masoni, P.; Buonamici, R.; Ekvall, T.; Rydberg, T. Life cycle assessment: Past, present, and future. Environmental Science and Technology 2011, 45, 90-96.

(17) de Jonge, M. M.; Daemen, J.; Loriaux, J. M.; Steinmann, Z. J.; Huijbregts, M. A. Life cycle carbon efficiency of Direct Air Capture systems with strong hydroxide sorbents. International Journal of Greenhouse Gas Control 2019, 80, 25-31. 
(18) Deutz, S.; Bardow, A. Life-cycle assessment of an industrial direct air capture process based on temperature-vacuum swing adsorption. Nature Energy 2021, 6, 203-213.

(19) Volkart, K.; Bauer, C.; Boulet, C. Life cycle assessment of carbon capture and storage in power generation and industry in Europe. International Journal of Greenhouse Gas Control 2013, 16, $91-106$.

(20) Holloway, S.; Karimjee, A.; Akai, M.; Pipatti, R.; Rypdal, K. Chapter 5: Carbon Dioxide Transport, Injection and Geological Storage. 2006.

(21) Kriegler, E. et al. Fossil-fueled development (SSP5): An energy and resource intensive scenario for the 21st century. Global Environmental Change 2017, 42, 297 - 315.

(22) Gambhir, A.; Rogelj, J.; Luderer, G.; Few, S.; Napp, T. Energy system changes in $1.5^{\circ} \mathrm{C}$, well below $2^{\circ} \mathrm{C}$ and $2^{\circ} \mathrm{C}$ scenarios. Energy Strategy Reviews 2019, 23, $69-80$.

(23) Luderer, G.; Leimbach, M.; Bauer, N.; Kriegler, E.; Baumstark, L.; Bertram, C.; Giannousakis, A.; Hilaire, J.; Klein, D.; Levesque, A., et al. Description of the REMIND model (Version 1.6). 2015; https : //ssrn . com/abstract=2697070.

(24) ISO, ISO 14040: Life Cycle Assessment, Principles and Framework. 2006.

(25) ISO, ISO 14044: Life cycle assessment - Requirements and guidelines. 2006.

(26) Liu, C. M.; Sandhu, N. K.; McCoy, S. T.; Bergerson, J. A. A life cycle assessment of greenhouse gas emissions from direct air capture and Fischer-Tropsch fuel production. Sustainable Energy Fuels 2020,-.

(27) Global CCS Institute, The global Status of CCS: 2010; 2011; Canberra, ISSN 18389481.

(28) Treyer, K.; Bauer, C. Life cycle inventories of electricity generation and power supply in version 3 of the ecoinvent database-part II: electricity markets. The International Journal of Life Cycle Assessment 2016, 21, 1255-1268. 
(29) Müller, L. J.; Kätelhön, A.; Bringezu, S.; McCoy, S.; Suh, S.; Edwards, R.; Sick, V.; Kaiser, S.; Cuéllar-Franca, R.; El Khamlichi, A., et al. The carbon footprint of the carbon feedstock CO 2. Energy \& Environmental Science 2020, 13, 2979-2992.

(30) Fazio, S.; Castellani, V.; Sala, S.; Schau, E. S.; M. Zampori, L. Supporting information to the characterisation factors of recommended EF Life Cycle Impact Assessment methods; EUR 28888 EN, European Commission, Ispra, 2018.

(31) Mutel, C. ReCiPe 2016 LCIA method for Brightway. 2020; https://github.com/ brightway-lca/bw_recipe_2016.

(32) Huijbregts, M. A.; Steinmann, Z.; Elshout, P.; Stam, G.; Verones, F.; Vieira, M.; Zijp, M.; van Zelm, R. ReCiPe 2016: A harmonized life cycle impact assessment method at midpoint and enpoint level - Report 1: characterization. 2016.

(33) Wernet, G.; Bauer, C.; Steubing, B.; Reinhard, J.; Moreno-Ruiz, E.; Weidema, B. The ecoinvent database version 3 (part I): overview and methodology. The International Journal of Life Cycle Assessment 2016, 21, 1218-1230.

(34) ecoinvent, ecoinvent 3.6. 2020; https://www.ecoinvent.org/database/ ecoinvent-36/ecoinvent-36.html, Accessed on Thu, June 25, 2020.

(35) Mutel, C. Brightway: An open source framework for Life Cycle Assessment. Journal of Open Source Software 2017, 2, 236.

(36) IRENA, Renewable Power Generation Costs in 2019; International Renewable Energy Agency, Abu Dhabi, 2020.

(37) Terlouw, T.; AlSkaif, T.; Bauer, C.; van Sark, W. Multi-objective optimization of energy arbitrage in community energy storage systems using different battery technologies. Applied Energy 2019, 239, 356-372. 
(38) Beltagy, H.; Mihoub, S.; Semmar, D.; Said, N. Feasibility Study of Linear Fresnel Solar Thermal Power Plant in Algeria. Africa-EU Renewable Energy Research and Innovation Symposium 2018 (RERIS 2018). Cham, 2018; pp 35-42.

(39) Industrial Solar, Industrial Solar. 2020; https://www. industrial-solar .de, Accessed on Thu, June $25,2020$.

(40) Schmidt, T. S.; Beuse, M.; Zhang, X.; Steffen, B.; Schneider, S. F.; Pena-Bello, A.; Bauer, C.; Parra, D. Additional Emissions and Cost from Storing Electricity in Stationary Battery Systems. Environmental Science $\mathcal{G}$ Technology 2019, 53, 3379-3390.

(41) Terlouw, T.; Zhang, X.; Bauer, C.; Alskaif, T. Towards the determination of metal criticality in home-based battery systems using a Life Cycle Assessment approach. Journal of Cleaner Production 2019, 221, 667-677.

(42) Arpagaus, C.; Bless, F.; Uhlmann, M.; Schiffmann, J.; Bertsch, S. S. High temperature heat pumps: Market overview, state of the art, research status, refrigerants, and application potentials. Energy 2018, 152, 985 - 1010.

(43) Austin, B. T.; Sumathy, K. Transcritical carbon dioxide heat pump systems: A review. Renewable and Sustainable Energy Reviews 2011, 15, 4013 - 4029.

(44) Sacchi, R.; Dirnaichner, A.; Terlouw, T.; Vandepaer, L.; Mutel, C. rmnd-lca. 2020; https://github.com/romainsacchi/rmnd-lca.

(45) Moore, Andrew D.,; Urmee, Tania,; Anda, Martin,; Walker, Elaine, Life cycle assessment of domestic heat pump hot water systems in Australia. Renew. Energy Environ. Sustain. 2017, 2, 38.

(46) Ma, S.; Jiang, M.; Tao, P.; Song, C.; Wu, J.; Wang, J.; Deng, T.; Shang, W. Temperature effect and thermal impact in lithium-ion batteries: A review. Progress in Natural Science: Materials International 2018, 28, $653-666$. 
(47) Luderer, G.; Pehl, M.; Arvesen, A.; Gibon, T.; Bodirsky, B. L.; de Boer, H. S.; Fricko, O.; Hejazi, M.; Humpenöder, F.; Iyer, G., et al. Environmental co-benefits and adverse side-effects of alternative power sector decarbonization strategies. Nature communications 2019, 10, 1-13.

(48) Hanna, R.; Abdulla, A.; Xu, Y.; Victor, D. G. Emergency deployment of direct air capture as a response to the climate crisis. Nature communications 2021, 12, 1-13. 
Life_Cycle_Assessment_of_Direct_Air_Carbon_Capture_...(7.28 MiB) view on ChemRxiv • download file 